\title{
The contribution of nursing to the health
}

\section{of New Zealand}

by

Jocelyn R Peach

A thesis submitted to Victoria University of Wellington

in fulfilment of the requirements for the degree of

Doctor of Philosophy

in Nursing

Victoria University of Wellington

2001 


\begin{abstract}
Nursing in New Zealand has been a recognised profession for one hundred years. Throughout this time the profession has made a significant contribution to the health of communities, nationally and internationally. Despite the obvious effort and achievement, the evidence of this contribution is not well known, is documented in a few literature sources only although it is talked about widely as part of 'myth and legend'.
\end{abstract}

Nurses, now as never before, are challenged to show how they 'add value' and to explain why nursing expertise is essential to safe service delivery. Finding a way to communicate this contribution has been identified as one of the most important issues facing the profession. This thesis explores the concept of contribution and presents a model, the 'Contribution Model', to show how nursing can articulate the action and achievements that show how nursing professionals have and will continue to contribute to health gain in New Zealand.

Through the application of the 'Contribution Model' and framework presented in this thesis, nursing is shown to have made a contribution to health gain by using the broad range of knowledge, skills and experiences in a wide range of settings, to provide care wherever and whenever required. Case studies and scenarios from history, observation and prediction are used to show how the actions and achievements of nursing meet the expectations of individuals, the community and society: past, present and future. 


\section{Acknowledgements}

I wish to acknowledge the encouragement and support I have received from family, friends, colleagues and academic staff over the past five years. They have supported me as my life has been 'on hold' while doing this thesis and balancing my workload commitments. I thank them for 'bearing vigil' with me, especially my mother who has been a significant supporter.

I would like to acknowledge in particular my supervisor, Cheryle Moss, who has encouraged me to work with my 'thought experiment' and offered unique and powerful insights. She has held me back so that I was forced to dig deeper, to find the 'points' of refinement, to 'maintain the line'. She has allowed me freedom to work around my all-consuming employment as a change manager. She has been accepting when my thesis hibernated and was reawakened at intervals. It is richer for the time to mature. Acknowledgement is also made of Pamela Wood for her enthusiasm and guidance in the final phases, her attention to detail and her patience.

Finally I would like to acknowledge my colleague, Andrew Jull, who was a guide to me in the early days of this thesis. He introduced me to scenario planning and we enjoyed hours of professional debate about the future, considering how scenario planning could be used to revolutionise the thinking of nurses. 


\section{The contribution of nursing to the health of New Zealand}

\section{Table of Contents}

Title page

Abstract 2

Acknowledgements 3

Table of Contents 4

Key to frequently used abbreviations and meanings $\quad 8$

$\begin{array}{lr}\text { List of figures and tables } & 9-10\end{array}$

Chapter One

The challenge for nursing to articulate its professional contribution $11-38$

Imperative for seeking a way to communicate contribution $\ldots \ldots \ldots \ldots \ldots, 14$

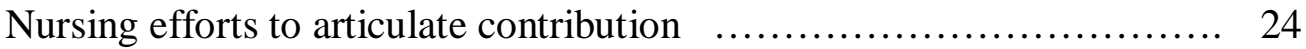

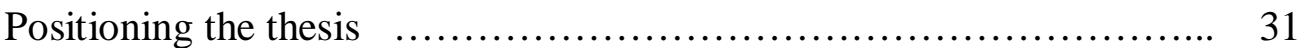

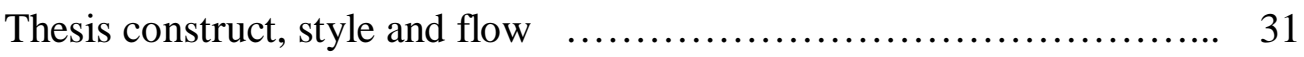

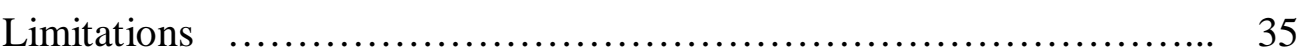

The influence of the researcher on the work $\ldots \ldots \ldots \ldots \ldots \ldots \ldots \ldots \ldots . \ldots \ldots$

\section{Chapter Two}

Contribution: the meaning and the theoretical base

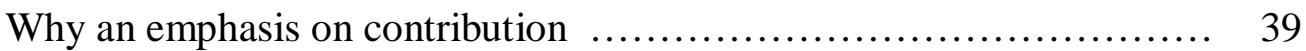

The profession seeking its voice and a sense of meaning $\ldots \ldots \ldots \ldots \ldots .41$

Contribution: achieving a common understanding of meaning as relevant 48 to nursing practice

Common themes identified as applying to nursing $\ldots \ldots \ldots \ldots \ldots \ldots \ldots .63$

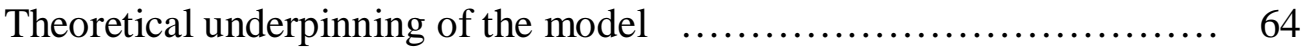




\section{Table of Contents, continued}

\section{Chapter Three}

Contribution as a conceptual model

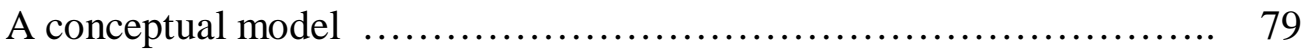

Pulling it all together: the model and the component parts $\ldots \ldots \ldots \ldots \ldots . \quad 88$

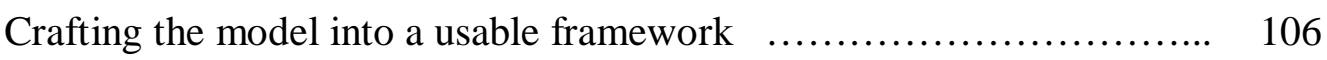

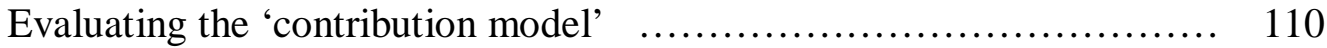

\section{Chapter Four}

Health: the constant priority of a nation

Why is health important? ......................................... 122

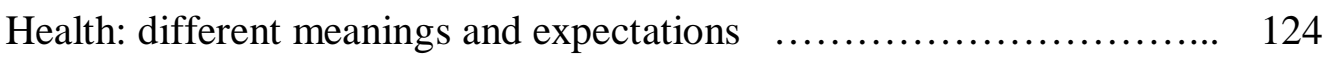

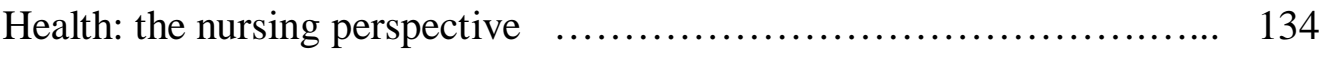

Health service priorities $\quad \ldots \ldots \ldots \ldots \ldots \ldots \ldots \ldots \ldots \ldots \ldots \ldots \ldots \ldots \ldots \ldots, 142$

\section{Chapter Five}

Nursing contribution: over time

Method

Use of case studies

The case studies

Case study 1. 1860 to 1900

Case study 2. 1900 to 1920

Case study 3. 1920 to 1945

The contribution of nursing during these periods in history 


\section{Table of Contents, continued}

\section{Chapter Six}

\section{Nursing contribution in the present}

Contemporary nursing and its contribution to health

Case study 4. Practice setting: Acute surgical nursing for breast 244 cancer.

Case study 5. Practice setting: Radiology nursing.

Case study 6. Practice setting: Inpatient adult mental health service.

Case study 7. Practice setting: Co-ordination of care for patients with chronic obstructive airway disease.

Case study 8. Practice setting: Operating theatre nursing.

Case study 9. Practice setting: District nursing.

The place and contribution of nursing in contemporary health delivery. 298

Nursing: a profession regaining confidence in the current environment 301

The modern image of nursing

\section{Chapter Seven}

Nursing contribution in the future

Scenario planning

Creating a scenario: the present leading into the future

Scenarios: "what if..." and the role for nursing

Scenario 1. Separate worlds (worse than now).

Scenario 2. Greater control (much the same with greater controls).

Scenario 3. Golden age of co-operation (better than).

Scenario 4. Moving forward to achieve the potential of development (radically different).

Implications of the scenarios for the role of nursing

So what might be the contribution for nursing in the future? 


\section{Table of Contents, continued}

\section{Chapter Eight}

The measure of contribution

The contribution of the concept 'contribution' 385

The contribution of the model to communicating the value of nursing to

health gain

The contribution of the conceptual model to nursing professional 390

knowledge

Challenging the profession to take action 395

What has been the nursing contribution to the health of New Zealand? 398

\section{Appendices}

Appendix 1 Nursing Competencies

406-408

References

409-437 


\section{Key to frequently used abbreviations and meanings}

\begin{tabular}{|l|l|}
\hline Abbreviations & Explanation \\
\hline AJHR & $\begin{array}{l}\text { Appendix to the Journals of the House of } \\
\text { Representatives }\end{array}$ \\
\hline NZPD & New Zealand Parliamentary Debates \\
\hline NZJH & New Zealand Journal of History \\
\hline
\end{tabular}

\begin{tabular}{|c|c|}
\hline Meanings & Explanation \\
\hline Kai Tiaki & $\begin{array}{l}\text { 'The Watcher - The Guardian'. The editors of The } \\
\text { Journal of the Nurses of New Zealand chose this title } \\
\text { because they perceived their role to be 'the guardian of } \\
\text { the helpless, the watcher over the sick and suffering' (Kai } \\
\text { Tiaki, 1908). }\end{array}$ \\
\hline Maori & Indigenous people of New Zealand \\
\hline Marae & $\begin{array}{l}\text { Courtyard, meeting house of Maori tradition. Traditional } \\
\text { practices and rituals govern visitor and community } \\
\text { behaviour when on the Marae }\end{array}$ \\
\hline $\mathrm{Pa}$ & Maori village, gathering of whare, or homes \\
\hline Tangata Whenua & People of the Land, first nation people \\
\hline Tohungia & Maori traditional blessing \\
\hline Whanau & Family \\
\hline
\end{tabular}

(Tauroa, 1990). 


\section{List of figures and tables}

\begin{tabular}{|c|c|c|}
\hline FIGURES & Title & Pages \\
\hline Figure 1 & $\begin{array}{l}\text { Complex adaptive systems in a system- } \\
\text { environment. }\end{array}$ & 73 \\
\hline Figure 2 & $\begin{array}{l}\text { Diagram depicting the flow of influence on the } \\
\text { agent. }\end{array}$ & 74 \\
\hline Figure 3 & $\begin{array}{l}\text { Complex adaptive system with nursing as a sub- } \\
\text { system and the 'Contribution Model' depicted. }\end{array}$ & 87 \\
\hline Figure 4 & Case study method. & 155 \\
\hline Figure 5 & $\begin{array}{l}\text { Complex adaptive system with nursing as a sub- } \\
\text { system and the 'Contribution Model' depicted. }\end{array}$ & 173 \\
\hline Figure 6 & Scenario development method. & 325 \\
\hline Figure 7 & $\begin{array}{l}\text { Scenarios based on driving forces and predicted } \\
\text { trends. }\end{array}$ & 345 \\
\hline
\end{tabular}

\begin{tabular}{|c|c|c|}
\hline TABLES & Title & Pages \\
\hline Table 1. & $\begin{array}{l}\text { Framework tool to use in applying the } \\
\text { 'Contribution Model' major units. }\end{array}$ & 107 \\
\hline Table 2. & Applications of the case study model. & 153 \\
\hline Table 3. & $\begin{array}{l}\text { Hospitals in the North Island of New Zealand in } \\
1897 .\end{array}$ & 169 \\
\hline Table 4. & $\begin{array}{l}\text { Hospitals in the South Island of New Zealand in } \\
1897 .\end{array}$ & 169 \\
\hline Table 5. & $\begin{array}{l}\text { Contribution framework for the period } 1860- \\
1900 .\end{array}$ & 174 \\
\hline Table 6. & Causes of death study in 1918. & 184 \\
\hline Table 7. & $\begin{array}{l}\text { Contribution framework for the period } 1900- \\
1920 .\end{array}$ & 204 \\
\hline Table 8. & Contribution framework for the period 1920-1945. & 228 \\
\hline Table 9. & $\begin{array}{l}\text { Settings and specialty areas where nurses work in } \\
\text { contemporary practice. }\end{array}$ & 239 \\
\hline Table 10. & Practice settings where case studies arise. & 243 \\
\hline Table 11. & $\begin{array}{l}\text { Contribution framework outlining the work of the } \\
\text { breast care nurse specialist role }\end{array}$ & 247 \\
\hline
\end{tabular}


List of figures and tables, continued

\begin{tabular}{|c|c|c|}
\hline TABLES & Title & Pages \\
\hline Table 12. & $\begin{array}{l}\text { Contribution framework outlining the work of the } \\
\text { nurse in radiology service. }\end{array}$ & 256 \\
\hline Table 13. & $\begin{array}{l}\text { Contribution framework outlining the work of the } \\
\text { nurse in the inpatient adult mental health service. }\end{array}$ & 264 \\
\hline Table 14. & $\begin{array}{l}\text { Contribution framework outlining the work of the } \\
\text { nurse working with people with chronic } \\
\text { obstructive airway disease (COAD). }\end{array}$ & 271 \\
\hline Table 15. & $\begin{array}{l}\text { Contribution framework outlining the work of the } \\
\text { nurse in the operating theatre. }\end{array}$ & 284 \\
\hline Table 16. & $\begin{array}{l}\text { Contribution framework outlining the work of the } \\
\text { district nurse. }\end{array}$ & 294 \\
\hline Table 17. & Driving forces & 339 \\
\hline Table 18. & $\begin{array}{l}\text { Contribution framework outlining the work of } \\
\text { nursing in the scenario of the world being worse } \\
\text { than now. }\end{array}$ & 352 \\
\hline Table 19. & $\begin{array}{l}\text { Contribution framework outlining the work of } \\
\text { nursing in the scenario of things are much the } \\
\text { same but with greater controls. }\end{array}$ & 358 \\
\hline Table 20. & $\begin{array}{l}\text { Contribution framework outlining the work of } \\
\text { nursing in the scenario of the world that is } \\
\text { radically different. }\end{array}$ & 370 \\
\hline
\end{tabular}




\section{Chapter One}

\section{The challenge for nursing to articulate its professional contribution}

Over the past two decades, nursing as a professional group within the subsystem of health service delivery and as members of New Zealand society, have been subject to major social and economic change, rationalisation and realignment. The changes have significantly impacted on the 'fabric' of society and the self-image of the profession. 'The New Zealand experiment', using a model of "pure neo-liberal economic theory" (Kelsey, 1995, p.vii), has resulted in significant structural adjustment, dis-aggregation and reconstitution of many social structures and focal practices. This has been a period of significant experimentation in the funding, organisation and management of hospitals. The application of management concepts and technologies has extended into strictly clinical domains with output-based funding mechanisms introduced and experimentation with methods for introducing value-for-money considerations into acute care funding (Degeling, Sage, Kennedy, Perkins \& Zhang, 1999).

The drive for efficiency and effectiveness has also been an international phenomenon, with "re-engineering and restructuring designed to emulate industrial models of productivity improvement rather than the creation of an environment that is conducive to high-quality care" (Aiken, Clarke, Sloane, Sochalski, Busse, Clarke, Giovannetti, Hunt, Rafferty \& Shamian, 2001, p. 51). The need for evidence of the value of contribution is in fact an international phenomenon as many countries have been impacted by the 
economic ideology demanding evidence of efficiency, effectiveness and benefit. In the focus on productivity and efficiency "individuals and groups have been challenged to provide evidence of the value of their contribution, to demonstrate efficiency, effectiveness and benefit" (American Nurses Association, 1998, p.2; McCloskey \& Bulechek, 1996). The need to articulate the function and value of nursing practice has been identified by many nurse leaders as one of the most important issues facing the profession as it "struggles to retain its identity in a health care system being restructured for greater efficiency" (Johnson \& Maas, 1997, p.6). The challenge for the profession has been to make visible its contribution as it has sought recognition of the important and pervasive role nursing has in the care component of health care and its effect on health outcomes.

For New Zealand nurses, the requirement to demonstrate contribution has been a particular challenge because of a cultural reticence to promote self and a limited number of post-graduate educated nurses prepared to confidently communicate the link between their activities and health gain. Limited professional leadership also had an impact as many nurse leaders were removed in restructuring or appointed to other general management roles.

As a member of the profession and as a nurse leader, I have sought ways to communicate my personal value and that of the profession. I have attempted to provide for managers, health planners and accountants a rationale for professional practice models and workforce numbers using my experience 
and my knowledge of the industry as well as using what evidence is available. Like many of my colleagues, I have had some success using case studies, exemplars and basic statistics, however, these approaches have been anecdotal, simplistic and of limited value.

This thesis therefore is the result of my search for a way to articulate professional contribution in a more systematic, structured manner. I have sought to create a simple model and framework that can be applied generically as it will be more valuable to practitioners than complex tools. I believe that it is possible and important to produce information using a common language that can be easily understood by a variety of stakeholders that can be used in discussions between the profession and health managers. The challenge has been to develop a simple, functional model and framework that can be used by individuals and groups, that has a strong theoretical base and produces information that is accurate and valuable.

In approaching this topic a number of assumptions underpin the thesis. The first premise is that it is possible to articulate contribution in a way that takes into consideration the complexity of different expectations; the diversity of practice settings and specialities; and that identifies outputs and outcomes that can be directly attributed to nursing intervention.

The second premise is that the contribution of individuals or groups can be explained and the value of that involvement made explicit using structured descriptions of a situation. It is assumed that by providing sufficient evidence 
and by linking outcome to expectations, the reader will be able to understand the benefit and value of the actions of the individual and group.

The third premise is that the 'Contribution Model' designed in this thesis is simple, flexible and can be applied in many situations. The very simplicity allows it to be demonstrated theoretically and does not require complex fieldtesting using traditional methodologies. The Contribution Model has been evaluated as a 'thought experiment', using case study and scenario-testing supported by data from written sources, observations about practice and scenario-thinking. The very simplicity of the model and framework reinforces its universal application as a tool to be used for communicating contribution.

This opening chapter positions the work in the socio-political context and identifies the problem that stimulated the research question that is answered in the body of the thesis that follows.

\section{Imperative for seeking a way to communicate contribution}

Professional imperative: In the 1980s, a mentor challenged me as a nurse leader to put in place structures and processes to assist nurses as professionals to develop confidence and strength to communicate their value and take charge of their professional destiny. This burden has motivated my actions for the past two decades. Amidst major structural change and placement of nursing leadership in advisory-influence roles rather than professional management positions, I have been part of initiatives to promote 
the development of professionals and to manage continuous improvement within nursing practice. In the health provider organisations where have I worked, the nurse leaders introduced a 'Professional Development Programme' that reinforced expectations for effective nursing practice at different levels of skill acquisition. The programme has structured support and established learning opportunities for all nurses. Nurses have been asked to prepare portfolios to profile their expertise and contribution. Through written exemplars and case studies, publishing and research, nurses have been encouraged to build their professional confidence in describing their contribution and their value. This has provided a way of communicating with the wider profession, as well as with administrators and health planners. While these initiatives have been generally successful in raising awareness and increasing confidence, there remains a lack of confidence in communicating with managers during structural changes, diffidence in expressing value and lack of clarity in the use of outcome measures to reinforce impact on health gain (Peach, 1999).

Nurses have expressed surprise that they need to explain what they do. Many acknowledge, however, that there is limited understanding of what nurses do. There is some frustration at the lack of valuing of professional involvement. "If nurses had to characterise the group to which they belong, they would start by saying that it (the professional input) is indispensable to society, yet that it is neither understood nor given due recognition by society" (Parrott, 1995, p.143). Nurses accept that they need to communicate what they do but find it difficult to describe their professional work and tend to either define it 
so generically as to be a universal description of caring or to describe tasks and actions.

Rarely is nursing described by practitioners in a way that links actions to the expectations of various stakeholders or to the specific needs within groups, although some of the significant literature has described the impact using outcome measures (Waltz \& Strickland, 1988; Waltz \& Strickland, 1990; Johnson \& Maas, 1997). The reliance on descriptions of actions and tasks to define nursing as a discipline has increased the vulnerability of the profession to initiatives by managers who dis-aggregate the work of nursing and delegated aspects to unqualified staff in the search for efficiency. The impact on nurses has been to create a sense of threat and perception of lack of valuing.

For some groups this has been of more significant concern than for others. While nurses can increasingly describe the 'difference' they make, they generally use qualitative descriptions of actions or use therapeutic goals undertaken by the nurse as practitioner rather than link the outcome to the specific health problem of the patient or family to emphasise the outcomes achieved. Outcomes, however, are increasingly the measures sought by managers and health planners as evidence of contribution and value and this is where nursing is least confident in its communication. Nursing is critiqued for having "enshrined process and ignored the evolving nature of knowledge, casting vision into myth and deifying process" (Kasper, 1995, p.62). Nursing is perceived as having embraced an ethic of pragmatism that values the 
practical and utilitarian completion of the task at hand. For nursing to assume the full range of modern practice it is challenged to integrate a comprehensive structural or expert knowledge that allows for "risk taking, a moral commitment to accountability and a tolerance for ambiguity" (Kasper, 1996, p.67).

The lack of confidence of many to express the outcomes of professional practice involvement has many possible origins. It may be due in part to the role of nursing as a complementary health team member where others prescribe care. It may be because of the dominance of medical classifications using disease entities to organise the thinking, speaking and writing of health teams. Or it may be attributed to the use of the "nursing process' problem-solving method that has emphasised procedures, tasks and functions and has been a tool emphasised in Western nursing education over the past twenty years (Gordon, 1998, p.4). Whatever the source of the lack of confidence, the need is for every practitioner to have a simple tool that they can use to understand their contribution and be able to articulate it using language that is clear and accurate.

Nursing in New Zealand has undergone a significant change over the past two decades as older senior leaders, prepared as nurses through the 1950s and 1960 s, have been replaced by nurses prepared since the 1970 s with a different preparation for leadership. Many of the new leaders have not progressed through traditional hierarchies and received limited mentorship. The current nurse leaders have been forced to justify their own existence, to 
establish systems to justify the cost of the professional workforce and to develop ways of communicating the potential and value of nursing to service delivery and health outcomes. The difficulty in articulating the 'what' and 'value' of nursing has been compounded by the pressures of the pace of social and technological change. Change has progressed quicker than the professional culture has been able to adapt to the requirements for quantifiable measurement. The profession's traditional communication style has been use of story-telling, 'doing' and action more than writing, reflection and research (Johnson \& Maas, 1997; Benner, Tanner, \& Chesla, 1996). Nurses have primarily used qualitative evidence in narrative and exemplar form to describe how they respond to a range of complex health, social and emotional needs, what their purpose was and the 'difference' they have made.

Structural imperative: New Zealand, as a relatively young and evolving society, has experienced two decades of major social and economic change, rationalisation and realignment that has had significant impact on the 'fabric' of the society. 'The New Zealand experiment' has impacted on most social structures, including the previously 'untouched' areas of health, education and social support services. The 'experiment' has impacted on individuals, their work and on the community structures. The technology of information management and general management has been used to search for efficiency and effectiveness, re-engineering many of the traditional approaches and forcing change to occur. These changes have had significant impact on individuals, communities and groups, as many of the systems and practices 
relied on have disappeared or been altered where they previously provided work, meaning and purpose to groups of people. Change has forced communities to re-look at the assumptions about the meaning of work and to review what could be expected from social support services. With the advent of 'user-pays' the concepts of 'added value' and personal responsibility have resurfaced, enforcing reflection by individuals and groups about how to measure productivity and how to justify contribution. Nursing as a service sub-system has been impact by this requirement to show value.

The structural changes over the past two decades have been rapid. New Zealand was a small, relaxed and relatively complacent population that had experienced a relatively high standard of living and quality of life. The wider society had anticipated that it would always stay the same, "it had developed a deep dependence on, and expectation of, major power paternalism" (Kelsey, 1995, p.19). Few predicted the extent of the economic and social change that occurred after the election of the Labour Government in 1984. The changes were aimed at establishing less reliance on government support and encouraging personal and commercial self-reliance. Many previously owned government agencies, such as railway, postage and telephone services were sold and some other government departments were developed into 'State Owned Enterprises' with greater emphasis on business management and a requirement to return a profit. Subsequent governments further developed this ideology so that New Zealand society has been altered significantly for many individuals and communities. 
While some of the changes have had a positive impact, recent evaluation suggests that there have also been consequences

the New Zealand experiment must be evaluated in its broader context, including the short-term effects of the process itself and its legacy for domestic economic life, social development, governance and cultural identity (Kelsey, 1995, p.11).

The impact on families and communities 'at risk' has been significant, which has in turn placed considerable pressure on health and social services as the impact on the social determinants of health have affected the socio-economic status and survival of individuals and communities.

It is not the purpose of this chapter to comment on the "model for structural adjustment" except to acknowledge its impact on the society within which nursing works and the consequent health needs that nurses seek to address. I acknowledge also that it was a stimulus for my search for a way to articulate the contribution of nursing to the health of New Zealand because of the need to explain the 'added value' of nursing as an essential component in health service provision. Nursing has been impacted on by the changes - as a profession and as individual practitioners who have borne witness to the impact of these changes on the individuals and communities that the profession supports.

The changes dismantled structures that had previously been the 'safety net' of free health services for all New Zealanders since 1935. The concept of 'user payment' for health services and of rationing, so that some services 
were no longer free, was a difficult concept for communities to grasp. The introduction of area health boards meant local regional governance of health services and an expectation of efficient management of services within annual financial allocation. Managers and accountants were required to manage within allocation and to seek more cost-effective professional workforce configurations, models of care and treatment methodologies in order to achieve the savings purported to be surplus in the system (Gibbs, 1987; Ministry of Health, 1992).

The introduction of 'user pays' altered the relationship between consumer and provider so that there was the increased challenge of what the provider could offer for the money being asked. Some health care procedures were also eliminated from that offered by the public health system and were available only if the user could pay a private health provider. These changes required individuals and provider groups in all sectors to find a way to communicate and measure their value as consumers were demanding more and so were managers. The demand for information was new as "critique is not a well-established tradition in this country" (Kelsey, 1995, p.vii). Information was not easily accessible and there were few validated measures established. Health provider individuals and groups had to learn new ways of describing, gathering and presenting information to in order to retain systems and processes and to communicate their contribution.

It has been a challenge for nursing and related health professions to justify the cost of their services, to explain the reason for retention of particular 
processes for safe practice and provide answers about outputs and outcomes (Johnson \& Maas, 1997). Providing evidence for managers has been particularly challenging. This was especially complex where the work of one discipline was closely integrated with that of other health team members and where measures could not be attributed to either group because the outcomes were a combined team effort. Measurement has been difficult where the action of an individual discipline could not be attributed to a single output using quantitative or output-oriented indicators. For example this occurs where the actions of nurses are diverse, generalised and specialised and have not easily fitted the measures or indicators favoured by economists, accountants and health planners.

Many of the managers appointed to general manager positions in the 1980s and 1990s entered the health service with little experience of health leadership but with years of commercial understanding and they demanded evidence and information that had previously not been requested. These managers used commercial management strategies and introduced methods of contracting and service planning to challenge existing roles, practices and processes where these had previously been taken for granted in health service delivery. These questions required nurses to explain what they did and why nurses were needed when unskilled cheaper workers might suffice. Nurse leaders were asked to answer why certain skill-mix variations were required for service delivery so that costs could be built into case-mix costing models. 
Considerable work had to be done to establish information systems so that these managers had adequate information to action recommendations and prioritise the issues requiring attention. Standard workforce information could be accessed, although this was not universal. Quantifiable measures have been difficult to find that adequately express the impact of these workforce groups on health outcomes. While some outcome measures were available and used to report to central government, these did not adequately reflect the complementary and integrated work of nursing within the wider health care team.

It was within this context that the research question arose for me: Could I create a structured model and framework that could be used to articulate the contribution that nursing has made to the health of New Zealand? Knowing that there are multiple understandings of the 'concept of contribution', could I develop a framework that might guide nurses in answering the demand for evidence of contribution?

Managers, health planners and accountants have asked for a variety of information that could be responded to as isolated requests or could be organised to provide a systematic description of the work done and outcomes achieved which was more aligned to the narrative way in which nursing communicated. Nurses were asked for explanations about the purpose of their work, how their actions were linked to the social expectations, why nurses were needed to undertake certain actions and what outcomes were achieved by certain actions. 
Through working to answer these questions, I have evolved a model and framework that is explained in the next chapter. The framework developed to make the model more user-friendly. The framework has been used in subsequent chapters to answer the question and to articulate the contribution of nursing in a number of case studies. The 'Contribution Model', as I have named it, has evolved in answer to the questions asked by managers, accountants and health planners and also to provide the profession with a tool that can be used to communicate with the various stakeholders.

\section{Nursing efforts to articulate contribution}

While much of health care is nursing care and "nursing plays a key role in the delivery of cost-effective care in every health care setting, it is imperative that nursing data is included in the evaluation of health care effectiveness" (Johnson \& Maas, 1997, p.xii). Without clear measures of outcome that can identify and measure nursing contribution, the profession of nursing will like other health disciplines be unable to identify outcomes that can be attributed to their professional input. "If nursing relies on physician-centered information only, the impact of nursing care will remain largely unmeasured and therefore invisible" (Johnson \& Maas, 1997, p.8). The impact and value of nursing care remains nearly invisible, under-recognised by the profession itself and by the health planners. This is evident in the questions that continue to be asked, such as: 'What is it that nurses do'? 'Do the actions of nurses make a difference to the quality of care received'? (Lower \& Burton, 1989; Mallison, 1990; Naylor, Munro, \& Brooten, 1991). Questions are asked about whether "the increased use of particular nursing actions result in 
the prevention of some medical conditions, the reduction of complications, or the enhancement of health and well being?" (McCloskey \& Bulechek, 1996, p.3). There is evidence of the value of nursing expertise in early detection and prompt intervention in studies relating to nursing staffing. It has been shown that variables such as nurse-to-patient ratios and nursing skill mix were significant correlates of inpatient mortality, especially when using indicators such as 'failure to rescue'. "A better understanding of which aspects of hospital care contribute most to improved patient outcomes could result in more effective use of resources as well as more effective care" (Sochalski, Aiken \& Fagin, 1997, OS20).

While the nursing profession in New Zealand and internationally has a responsibility to communicate answers to these questions, this has not been undertaken comprehensively. While nursing has grappled with these questions the result has been to describe nursing in a global manner that does not clearly differentiate the work of nursing from that of other helping disciplines (New Zealand Nurses Organisation, 1993). Alternatively nursing actions have been described so specifically as a plethora of interventions that it is overwhelming to the community and health planners who seek a succinct and simple definition (McCloskey \& Bulechek, 1996). The need for a clear description of the purpose of nursing will be explored in later chapters.

The knowledge and skill of nursing is described in the report of the New Zealand Ministerial Taskforce on Nursing (1998) as making "a distinctive contribution to the delivery of health care and client outcomes". The 
narrative goes on to describe nursing as practised "in child-health services, residential-care facilities, mental-health services, community services, Marae, independent-nurse clinics, public-health services..." (Ministry of Health, 1998, p.21). The report describes nursing as

a combination of many elements: knowledge, styles and models of care, professional codes, clinical skills and attitudes. Central to nursing is the client relationship that emphasises continuity of services and care and provides monitoring and evaluation of all health-care components for that client. In everyday practice, nurses provide a unique contribution to care outcomes though a presence and thoughtful assessment, planning, implementation and evaluation activities which are focused on the 24 hour experience of patients and their families (Ministry of Health, 1998, p.22).

These general descriptions assume that nursing contribution is understood, that the purpose of nursing is clear to the practitioners of nursing and the receivers of practice actions. The authors acknowledge that "it is not straightforward to measure the total contribution nursing makes to health outcomes" (Ministry of Health, 1998, p.24). The writers make no suggestions about how this difficulty might be addressed, nor do they comment on the importance of measuring the contribution. Instead, there is an inherent assumption that the various stakeholders understand what nursing contributes and that what nursing does is valued without the need to quantify what that value is to health outcome. I believe that nursing can no longer work without 
evidence of contribution and that it is our professional responsibility to seek and present the evidence.

The profession continues through internal discussions and narrative to sustain "the informal culture of attitude and practice" that supports the fragmentation of tasks, identifying nursing purpose in description of tasks or interventions and reacting when tasks are delegated by managers to unqualified workers. Focus on tasks lays the basis for deskilling. Breaking down the work of nursing into tasks preserves the subordination and powerlessness of the functions undertaken by nurses. The profession however emphasises the systematic body of professional knowledge as the differentiator of nursing contribution (Turner, 1987, p.152) but does little to show how the knowledge is applied to achieve the expected outcomes. Nursing asserts that it makes an independent contribution and it is important to the therapeutic process. The evidence of this contribution is difficult to demonstrate however without a systematic framework for presenting supporting information. I believe that a profession can be said to have 'come of age' when: it can clearly articulate its purpose; when it can demonstrate its contribution in a consistent and credible manner through research evidence; when the members of the profession are confident about their value and place in social expectation; and when they act to consistently provide what is expected.

Despite the profession asking questions about the meaning of its own work, nursing is still developing a theoretical tradition to assist with articulating its purpose and value in language and measures that can be understood by health 
planners. Some work has been done in the past decade by various research groups to align international nursing language to the Diagnostic Related Groups and health conditions used to diagnose and treat patient needs. Work has been done by the International Classification for Nursing Practice project (Mortensen, 1996), the North American Nursing Diagnosis Association's (NANDA), and the Nursing Interventions Classification (McCloskey \& Bulechek, 1996). The widespread use of NANDA's nursing diagnosis language has increased awareness of the need for standardised classifications, interventions and outcomes. The impetus for 'Guidelines Development', under the leadership of the New Zealand Guidelines Group and the Cochrane Collaboration, has further clarified the need for a universal language and for measures of outcome.

The various activities cumulatively seek: to develop standardised nomenclature of nursing and health treatments; to expand nursing knowledge about the links between diagnosis, treatments and outcomes; to develop information systems that will allow for analysis; to teach decision making; to determine nursing costs; to allocate nursing resources; to communicate nursing to non-nurses; and to align nursing knowledge articulation with the classification systems of other health care providers (McCloskey \& Bulechek, 1996, p.15). The stated goal of all of this work done internationally has been so that the profession could clearly articulate and make visible what nurses' do that was previously invisible. Much of this work however focuses on in-depth descriptions of the actions of nurses. The work does not guide the practitioner or profession to clarify the purpose of 
nursing in relation to expectations of the communities served. It does not show how the actions meet the expectations or why the professional response to other factors might alter the actions taken. The study method continues the traditional focus on describing the actions of nursing. While the interventions and outcome descriptions can be described to show a link between the interventions and outcomes, in a 'cause and effect' method, there is benefit in articulating the action and achievements against a systematic framework that allows the context of practice to be understood.

Being able to articulate contribution is essential to the survival and credibility of a profession. A profession that has a strong sense of its place in meeting the expectations and needs of a society will be able to recruit new members, plan and develop the range of responses appropriate to the current and future needs and contribute to strategic planning. Having a language that is widely understood and a way to articulate 'contribution' is important for 'internal' re-focussing as well as external profiling. Despite the increasing tertiary postgraduate education of nurses in current practice, they still require encouragement to articulate clinical thinking, anticipatory decision making and outcomes. Some nurses resist the use of taxonomies, simple categorisation or protocols (Benner, Hooper-Lyriakidis \& Stannard, 1999). They encourage "narrative understanding of the specific situation with reflection to allow the caregiver to use scientific knowledge melded and suffused by the time, place and conditions of care, which in turn leads to clinical wisdom" (Lynaugh, 1999, p.vi). While the narrative methodology is valuable and is intuitively understood by nurses, the information does not 
extend sufficiently to provide qualitative and quantitative evidence that can be used to communicate with managers and accountants. Alternative methods are urgently required to provide the evidence and it falls to nurse leaders and educators to continue to seek ways to encourage practitioners to communicate about their work in a positive and constructive manner, to encourage documentation that clearly establishes the trail between need, action and achievement. It is essential to find a way that achieves both professional imperatives and structural needs. How this is achieved is still to be worked through but it may be through nurse leaders who are expected to "design organisational systems needed by clinical nurses to provide them with the authority and control over their practice as they act on behalf of patients" (Clifford, 1999, p.vii).

While change and management ideology in the past decade has demanded that nursing provide evidence of the value of professional activity, nurses in New Zealand have been slow to use international nomenclature from research or to develop their confidence in presenting meaningful evidence of their contribution. The Ministerial Taskforce on Nursing identified that it is possible to measure and cost the contribution nursing makes to the delivery of health and disability services and patient outcomes (Ministry of Health, 1998, p.24), yet there were few recommendations for how this might be articulated. Work has been undertaken internationally by nurse researchers (Benner, 1984; Benner, Hooper-Kyriakidis, \& Stannard, 1999; McCloskey \& Bulechek, 1996; Johnson \& Maas, 1997; International Council of Nursing 
2001). These initiatives appear to have impacted little on nurses in New Zealand, but could be integrated as will be shown in later chapters.

\section{Positioning this thesis}

This thesis is about nursing and the work of nursing, grounded in clinical practice and research. The 'Contribution Model' arising from this thought experiment has been developed to assist nursing articulate its contribution, using clear, clinically useful language. The work acts as a 'discloser' of the world of nursing and 'preserver' of the 'essence of being' of nursing. It describes the 'connectedness' of nursing action to the expectations of society. It shows the links of the actions and interventions used by nursing to meet the needs of individuals and communities. It identifies how the achievements can be attributed to nursing. It reflects on the responsiveness of the profession to the changing social expectations and health events. This thesis also acts as a statement of philosophical resistance to the 'technology' of economic rationalism that favours dis-aggregation in search of efficiency rather than valuing integration and continuity. This work is significant for professional practice. Being able to articulate how the profession 'adds value' represents the greatest opportunity for nurses to define and develop their professional status, for to remain silent signals the onset of the disintegration of the profession as it is now known (Parfitt, 1995).

\section{Thesis construct, style and flow}

The thesis is constructed in three parts:

Part One sets the context and presents the model, the assumptions and elements underpinning the model components that assist with articulating the 
concept 'contribution'. This section also considers the context of health, within which nursing works with individuals and communities and explores the changing expectations of society as regards health, health priorities and the expectations of health professions. Part Two applies the model using different time periods to demonstrate how the contribution of nursing to the health of New Zealand can be articulated. The model is applied across the three dimensions of history, contemporary practice and predicted future contribution by nursing. Case studies and scenarios are used to show how the work of nursing has, does and will continue to 'add value'. Part Three concludes this theoretical work with an analysis of contribution from three perspectives: the contribution of the 'Model' to communicate the value of nursing to health gain; the contribution of the model to nursing knowledge and the profession; and the nursing contribution to the health of New Zealand.

The thesis is constructed in seven chapters.

Chapter One provides an introduction to the underlying imperatives for addressing this topic and sets the scene. In Chapter Two and Three, the meaning and assumptions underpinning the concept 'contribution' are explored as a potential source of elements to construct a model for communicating the contribution of nursing to health gain. The patterns and themes arising from this analysis inform the components of the model that are presented and applied throughout the rest of the thesis. The theoretical base of the model is explained and the 'Contribution Model' components are described. 
In Chapter Four, the concept of health is explored, as health is the context within which nursing works on behalf of society and its members. It is essential therefore that the work of nursing is linked to the strategies of the society within which the profession acts to contribute. The expectations of society as regards health and determinants of health inform the priorities for action by professionals working in health. These priorities change over time as events arise and as scientific developments alter practice to minimise disease incidence and offer different treatment options. Good health enables people to participate more fully in society and contribute to community productivity by pursuing their goals in life. It is therefore the aim of all to achieve good health and participate in a good life, regardless of disability.

In Chapter Five, the 'Contribution Model' is applied using a case study method and information from three time periods in early New Zealand history. Through the use of case studies, the expectations, actions and achievements of nursing are viewed using a three dimensional perspective to consider 'what was happening in society'; 'what was happening in health'; and 'what nursing was doing to respond'. This provides a way of presenting a word picture to communicate how the actions of nursing meet the expectations of society to achieve the expectations that are anticipated by various stakeholders.

In Chapter Six, the 'Contribution Model' is again applied to contemporary reality and observed nursing practice. A number of theoretical case studies 
are developed from observation of nursing practice in diverse practice specialities. The application of the 'contribution model' shows how these specialty roles might articulate what they do and the scope and complexity of their function. Some of the roles are under some level of perceived threat to be replaced by other providers and nurses are not clearly able to articulate their contribution. The six case studies are developed using observation and knowledge of acute nursing in inpatient and community settings such as working with women requiring breast surgery for cancer; nursing roles in the operating room and in radiology clinical settings; nursing in Mental Health in-patient setting and district nursing; and specialist nursing coordination for people with chronic obstructive airway disease. The 'Contribution Model' is used for each case study to demonstrate how the model and framework can assist in communicating the work of working in these speciality roles.

In Chapter Seven, the contribution of nursing into the future is explored using scenarios and a 'futures thinking' methodology. The question to be answered is what could happen over the next twenty years and what will the nursing profession do to contribute to the health of New Zealand in any of the possible future scenarios? This is an important discussion because without an understanding of the potential changes that might arise from anticipated environment adaptations, developments of technology and globalisation, the profession is likely to be more reactive than proactive. Informed anticipation allows planning for the education of future 
practitioners, planning for future workforce requirements and recognising the role nursing can have.

In Chapter Eight, the contribution of this theoretical work is considered: the contribution of the 'Model' to communicate the value of nursing to health gain; the contribution of the model to nursing knowledge and the profession; and the nursing contribution to the health of New Zealand. This chapter includes a challenge for the public and the profession to value and protect the treasure that is nursing and to acknowledge the value of its contribution to health gain.

\section{Limitations}

There are limitations in this theoretical 'thought experiment'. The reliability of events used in Chapter Five are limited to reliance on written descriptions by nurses of their work, as documented in journals and publications by historians who have written from different perspectives and use of archival records from the Ministry of Health annual reports. These descriptions are subjective and cannot be formally validated, as they were the only sources found from that time period. The descriptions are also reflections of the culture of the time period with the prejudice and stilted correctness that was indicative of these historical periods. The descriptions in Chapter Six are derived from personal observation and experience and exposure to the work and practices of nurses in these settings. These observations have not been validated with practitioners in current practice and so should be read with this in mind and not critiqued as necessarily being technically accurate, as this is not their purpose. Greater accuracy could be generated by focus group 
analysis involving contemporary practitioners but this was not deemed necessary by my supervisors. The reason for using these case studies as applied to the 'Contribution Model' and framework is because they show how the model can be used to assist in articulating the scope of practice succinctly. In Chapter Seven, the ideas presented in the four scenarios are derived using my imagination, from review of the literature, from reports in journals and review of Internet websites advising of developing science and technology. The final limitation is that the case studies and scenarios have been applied theoretically rather than using field-testing.

\section{The influence of the researcher on the work}

I have been a nurse leader for the past twenty-six years, working amidst waves of structural change in health service configuration: from Hospital Board to Area Health Board to Crown Health Enterprise to Regional Hospital and Health Service and latterly District Health Board. This has also been a period of considerable change in the scope of professional practice, specialisation of practice settings and diversity of nursing and allied health roles.

Throughout this time I have felt a strong sense of accountability as I have influenced the way in which professionals have developed, articulated their value and expanded their knowledge and expertise. I studied the techniques of management through a Masters-level programme in order to have the language and expertise to act as 'translator' to both managers and professionals. I introduced a Professional Development and Recognition 
Programme ("PDRP”) across the Auckland region from 1986, with the purpose of assisting nurses gain awareness, skill and confidence in communication of the 'what' of their nursing practice and seeking to profile the difference their practice made. The "PDP" concepts have subsequently been adopted in many local public and private health provider settings in the region. The concepts have also been used nationally and many of the ideas have been included in the Nursing Council of New Zealand requirements for competency-based practising certificates 2000 (Nursing Council of New Zealand (2000).

Nurses have been slow to respond to the leadership and encouragement to profile their practice and to state clearly what difference they make. Many continue to respond with anger or helplessness to the increasingly commercial, litigious, clinically and professionally demanding environment. It is hoped that the 'Contribution Model', arising from this thesis, will be viewed by nurses as a way to identify their purpose and confidently communicate their value. The 'Contribution Model' has the potential to "guard the traditional past, act as a guide to the present, create and inspire the future" (Newton, 1965, p.20). Toynbee's theory of challenge and response suggests that where new large-scale challenges arise, civilisations respond to sustain their existence through their creative development which are often the consequences of their prior development. In that spirit, challenges are opportunities to improve, by exercising the attention, understanding and ultimate creativity (Toynbee, 1934). The 'Contribution Model' and the 
associated framework provides a creative way of responding to the challenge to articulate professional value to meet expectations.

This first chapter has provided an introduction to the socio-political context that has influenced the development of this thesis. Since the 1980s New Zealand has experienced a social revolution as structures and processes have been altered in response to ideological decisions. Amidst this change, nursing has worked with those impacted by the changes but has also been influenced as individuals and professionals by the changes. The demand for nursing to justify its contribution has challenged the profession. Nursing has not developed a consistent way to communicate what it does or ways to measure the value of the contribution to health outcome. The next two chapters present a simple and practical tool that can assist nurses communicate their contribution. Through analysis and interpretation of the word 'contribution', a model and framework has evolved. The 'Contribution Model' is applied in the middle chapters to demonstrate its usefulness and efficacy. The 'Contribution Model' and framework tool provides a way to profile contribution and the significant value that nurses offer as an integral component in the provision of health services to New Zealand. It is clear that without nursing much of the progress of the past century would not have been achieved. Communicating this value and benefit, therefore, is important to the identity and future place of nursing in health service provision. 


\section{Chapter Two}

\section{Contribution: the meaning and theoretical base}

The word 'contribution' has entered common usage in the past two decades, used in a value-laden way to depict something good and of value. The word has also been applied to individuals and services as a descriptor of 'added value', as a way of explaining how they 'make a difference', using measures to show that actions and observable output or outcomes can be attributed to their practice (Johnson and Maas, 1997). In this chapter, the interpretations and meanings associated with the word 'contribution' are examined, as they influence the development the concept of contribution and the implications for nursing. The patterns and themes arising from the analysis of the contribution concept is important, because they form the basis of the 'Contribution model' that is applied in later chapters. This chapter is significant because it explains the origin and theoretical base of the model that is a foundation for the structure of the 'Contribution model'.

\section{Why an emphasis on contribution}

The emphasis on contribution, as a descriptor of something importance and value, has arisen over the past few decades from the ideological economic reforms and introduction of the model of general management. Managers have increasingly focused on the "contribution ratio" of various components such as roles, services or departments, as an indicator of the fiscal viability of an organisation and its productivity. For many individuals and groups, the ability to find measures and to articulate individual and specific value has 
been difficult, either because their work is integrated with that of other team members or they have not developed processes, a language or confidence to explain their purpose, function and achievements. The concept of 'contribution' has not appeared to have a common meaning or measure amongst all groups although the term is used generically as applying to the expectations of health or social agencies and is used by the economists and social political engineers to denote something of value. The word 'contribution' in common meaning also implies action and responsiveness. This is relevant to the observed history of the nursing profession which, from its modern-day inception, has been active in response to the needs and expectations of many groups. Contribution also implies a collective input and this resonates with the reality of professional nursing practice because nurses have always and continue to work in collaborative integrated teams providing multiple inputs and they less commonly act in isolation. The generally used meaning of the word, while understood philosophically by society and professionals, does not have the same meaning or measurement across all groups. It was considered important therefore to seek to develop a common set of meanings and a consistent way of measuring the concept so that there could be a shared understanding and communication to allow for effective discussion and documentation of evidence.

I was initially drawn to explore the meaning of the word 'contribution' because of its value-laden use, the inference that to contribute was something good, to be achieved in order to be taken seriously. The concept appeared to have layers of meaning and it was thought that through understanding the 
multiple meanings of the word there might be a way to communicate and negotiate an approach to valuing nursing contribution. From review of the various dictionary meanings and exploring the assumptions and interpretations that could be made from the definitions, it was clear that the concept could be applied to contemporary nursing practice issues. The themes arising from the interpretations were also identified as able to be framed into a model that could be further designed into a framework. When applied to various situations it was clear that the user-friendly and functional model made it easier to articulate the contribution of nursing regardless of the variety of diverse contexts. From the word 'contribution' has evolved a model that has been applied throughout this thesis to show how it assists with structuring and facilitating a clearer description of nursing in action.

\section{The profession seeking its voice and a sense of meaning}

Nursing as an acknowledged profession is a modern phenomenon although many of the functions of nursing can be found to have been part of social consciousness and actions for thousands of years (Austin, 1957; Donahue, 1985). The development of the modern practice of nursing in New Zealand arose in the 1800s during a period of rapid social and industrial development. Factors influencing change included increased social reform and international colonisation where ideas from Great Britain and Europe were imposed across the world, regardless of existing cultural practices. Educated and skilled people were in high demand across the developing world and they were expected to demonstrate professionalisation and accountability in their roles in society, this expectation becoming stronger as science and 
technology developed. New social structures were established and the need for healthy communities was viewed as essential for social and economic growth, stability and survival. There was increasing use of science and epidemiological evidence that where poor health outcomes existed, links could be shown between environmental hygiene and ill health.

During this time, nursing was a developing practice, organised under the leadership of strong women such as Florence Nightingale (Britain) and Grace Neill (New Zealand). Doctors also supported the development of modern nursing as they acknowledged the need for and value of quality nursing practice to provide support for their medical practice. There was considerable effort made to attract middle class women to nursing, to eliminate the reputation of nurses being uneducated and undisciplined and to gain respect as highly disciplined, educated and accountable practitioners. This was a dynamic time for nursing.

Nursing as a new profession prepared in an apprenticeship model, with probationers working long hours, many leaving the training programme or soon after registration, as they married and had children. Registered nurses, in short supply and in high demand, were asked to work in many settings to provide health care. The need and demand for nurses appears to have been accepted with few questions. Health planners and politicians were clear what they wanted from nursing as a social agency: "health missionaries to go forth to care for the sick, to lecture and to uplift humanity (AJHR, 1908, p.119). 
Communities accepted the presence of the nurse, valuing the roles they undertook (AJHR, 1912, p.21).

The need at that time to articulate and communicate the value of nursing to the health of the community was not seen as important. It is ironic therefore that the contribution of nursing is more evident in historical literature than in current New Zealand publications. While these nurses could be considered less academically educated than the modern nurse, the pioneer nurses were eager to extend their learning and they communicated extensively through the quarterly publication of the professional journal launched in 1908. They used exemplars of their practice to describe their experiences, perceptions and aspirations. This writing was informative, qualitative and narrative in style, similar to the storytelling that has interested nurses through the decades, fostering socialised professionals. This narrative approach is of limited value to managers or health planners seeking to prove achievement of outcomes. The writing by nurses in these early journals does not appear to have been aimed at providing evidence of contribution or proving value.

The descriptions of practice in the early years were detailed and holistic, with nurses describing the variety of activities undertaken, not differentiating practice as individual or speciality tasks. Nursing practice was described as a broad scope of practice, as groups of actions that achieved outcomes whether it was recovery or a peaceful death. Even the annual reports of the Department of Health from 1897 to 1950 described, in some detail, the range and scope of nursing involvement and relevant issues in answer to questions 
of interested politicians and communities. After the Second World War, nursing documentation in The Journal of the Nurses of New Zealand demonstrates increasing specialisation in practice. Other health team members appear to emerge to undertake aspects of the work previously the domain of nurses such as social workers, dieticians and physiotherapists, to use their modern titles.

The annual reports of the Department of Health appear increasingly silent from the 1950s about the work of nursing or other health disciplines. From the 1990s the annual reports make no reference to the work of professional groups and have focused on the efficient use of taxpayer money and expectations of health services providers to achieve identified health goals (Ministry of Health, 1998).

Workforce shortages appear as a regular commentary in The Journal of the Nurses of New Zealand and in government reports through the first sixty years. It was acknowledged that health service providers, whether organisations, groups or individuals, could not achieve expectations if there was a short supply of workers. In the Department of Health reports, there was continual reference to recruitment of probationers and the "shortage of senior qualified nurses...gaps filled by nurses by recently qualified and without experience" (Maclean, 1918, p.212; AJHR, H-31, 1918, p.10).

Over the past fifty year period, the education and preparation of nurses has altered. The learning models have followed the medical bio psychosocial 
systems approach and have become increasingly specialised. Nursing care practices have become less generic, no longer to be applied to meet the needs of any person across the health service continuum, as the early pioneer nurses had practised in the care for the sick, prevention of disease and promotion of health. Science and technological developments have increasingly specialised the practice of modern nursing. The diversity of practice has also expanded, differentiated by specialist scopes of practice that have mirrored the increasing specialist practice of medicine.

Over time, doctors have delegated speciality tasks to nurses whom they have known and trusted, nurses they have personally educated in the speciality inservice education programmes such as neonatal, cardio-thoracic, intensive care and coronary care nursing programmes. Nursing practice has become less generalist and more specialised across an episode of care (Benner, Tanner, \& Chesla, 1996; Benner, Hooper-Kyriakidis, \& Stannard, 1999). For example, specialist nurses focus on the care of patients/client with urological, neurological or haematological health problems and not on general medical or surgical nursing scopes of practice. My experience is that contemporary nurses are observed to identify more with their practice within the speciality and the specialist tasks undertaken. They appear less confident in caring for a patient with other co-morbidities or in other speciality practice settings. The patients also describe the specialised work of nursing, such as intensive care or emergency nursing, as they perceive and witness the actions taken in that episode of care. Patients rarely see the generalist nurse across the continuum of family-centred care. Even public health nurse work is now specialised into 
areas of focus, such as 'under five assessment' or school health, and is more constrained in scope than was the case in the establishment period of community nursing.

Where previously the community understood nursing to be wide-ranging and inclusive, they now experience specialised and exclusive. The patient rarely experiences continuity but is referred to another team or specialist for specialised assessment and care planning. The nurses describe their practice in relation to the speciality and the procedures rather than describing the nursing contribution overall in that setting. The patient and family are not always able to see the full range or core consistency of nursing practice, as many of the practices, such as prolonged bed rest for convalescence have been replaced by non-invasive procedures, day procedures, early perambulation and early discharge. Nursing practice has changed over the past thirty years with fast-track procedures, shorter inpatient care episodes, reduced length of stay and increased complexity in the care provided as more is done through resuscitation and treatment than was done previously.

Ironically, while nurses in New Zealand have been increasingly tertiaryeducated in recent decades, publication of research has been slow to flourish in New Zealand and few New Zealand nurses have been published in international refereed journals. Nurses seem reticent to articulate what nursing is, why nursing is necessary and what contribution nursing makes. Adam contends that this is not because they "do not have a conception of nursing but because their conceptual base is not clear. If the nurse's mental 
image of nursing is vague or blurred, it will therefore be difficult to put into words" (Harbour, Creekmur, DeFelice, Doub, Hodel, Tomey, Petty, 1998, p. 517). Few nurses appear to have confidence to speak publicly on a broad range of issues about the health of communities. This could be in part because of their narrower speciality focus, less opportunity, the role of the media and employer restrictions in an increasingly litigious environment. The media often seek medical specialist opinion as a traditional reference on health issues than other health care professionals. In many instances nurse representatives are not included as of right, as would appear to be the case in other countries, such as the United States of America or Australia. Exclusion may be because contemporary health planners and politicians do not appreciate that nurses have a unique understanding of community and health issues, or that nurses can recommend economic solutions. As an observation, the nursing voice on health advisory groups or in the media is nearly silent, except for bemoaning its absence 'at the decision-making table' (Ministerial Taskforce on Nursing, 1998).

The majority of the profession provide nursing care from within their speciality 'silos', threatened by the advent of generic support workers or specialist other workers who might assume some of the tasks currently undertaken by nursing. Nurses remain silent, relying on nurse leaders and union groups to explain the value of nursing, to ensure that the mix of workforce is maintained, to debate the limit of unskilled workforce numbers to qualified staff because of safety and quality issues. In reality it is not always the orchestrated response of nurse leaders that makes the most impact, but the confidence and clarity of each practitioner able to 
communicate their particular purpose, role, function and achievement. If nursing is to demonstrate its social responsibility, then nurses must be confident to articulate the needs of the communities they serve. This confidence is developed through education, practice and systematic thinking processes.

\section{Contribution: achieving a common understanding of meaning as relevant to nursing practice}

There are multiple meanings of 'contribution' in contemporary use. In the finance context, accountants use to contribution to mean the reported performance of each individual, department or component to the budget forecast and pre-set indicators. Contribution monitors the impact of subsystems on the financial outcome of the larger system. In a social service context, it is used to mean the effort made in terms of time and activity towards a common goal. In media briefings and political comment, contribution is used to describe involvement in an important activity over time, positive input on an issue perceived as beneficial. While all of these meanings are relevant in the situation to which they apply, I have referred to the dictionary to find a formal meaning that has international meaning and is consistent. The dictionary definitions suggest that 'contribution' is defined as:

to give jointly with others to a common purpose; to give (money, an idea, help) towards a common purpose; to help to bring about a result; supply (an article for publication) with others; the act of contributing; to be partly responsible for, to help bring about, action 
in the aid of a common effort; to give (money, support, help or ideas) towards a particular aim or purpose; individual efforts in a common endeavour; act of giving in common with others for a common purpose.

(Little Oxford Dictionary, 1998, p.135; The Concise Oxford Dictionary, 1990, p.250; Cambridge Dictionaries 2000).

These definitions imply that action is purposeful and goal-directed, that it is linked to a named purpose or project, that the action is valuable to the overall outcome and that there is a tangible output to add value to the situation or collective work. While the action appears to be focused, when action is applied to a specific situation, the actions may be multiple depending on the expectation, the skills of the contributor and the requirement of the time and place.

In the following section, the interpretations and application of the meaning to nursing will be explored in more detail using the main elements of the definition outlined above. The elements arising from the definitions will be used to develop interpretations that apply to nursing. The elements used are: 'acting to support a common purpose'; 'to give jointly'; 'to supply ideas or opinions'; 'to be partly responsible for' and 'to help bring about something'; 'action in aid of a common effort'; 'to bring about a result'; 'the act of contributing'.

Acting to support a common purpose. This element implies an understanding of the common need and aspirations inherent in being part of 
the group and having an accepted role within which to act. The activity of nursing has been present to support the common purpose of the community as far back as can be identified.

Health has been important for communities from ancient times (Donahue, 1985). Treatment of illnesses and introduction of sanitary practices to prevent health problems evolved over time and in response to understanding of the cause and effect of certain illnesses, uses of plants, elements and practices as well as scientific developments. The assumption of particular roles within the social group, as an expectation of social membership, has existed from early civilisations with certain roles used to maintain social order, to ensure protection, nurturing and survival of a community (Donahue, 1985, p.17). It is not known but it is assumed that roles were probably not formally articulated, negotiated or allocated, but that members with particular skills and abilities undertook specific functions required by the group to provide for the greater good. Over time particular roles were acknowledged as having life-giving powers and granted a place of prestige and protection, especially as magical lore became more complex requiring arduous training.

Regardless of the size of the community or the time period in history, certain members of the group were always required to provide care for those who were vulnerable and in need of assistance because of illness or age. Women, either as mothers, slaves or domestics, were called upon to provide care for childhood illnesses, applying these skills to other related situations for other community members. Associated with the 'medicine men' were a group of 
practitioners, usually women of the tribe who applied treatments, ascertained quantities of medicines, dressed wounds and reduced fevers. They used medicinal herbs and other simple treatments learned and handed on over time. The work of these women expanded to include work in concern for other human conditions such as poverty, prevention of disease and helplessness. Contribution was a requirement of community membership and certain members undertook work to provide nurture and support. They acted to support the common effort of maintaining the survival of the group.

The origins of nursing in the past millennium show that it was predominantly women who were identified as nurses, providing experienced support and assistance for members of the community who were vulnerable, poor or without social supports (Donahue, 1985; Austin, 1957). The activities of nursing from the ancient Egyptian era suggest that temples had some type of housing for the sick. In the early Christian period nursing occurred either in the home or in places of group caring, called a xenodochia, a house for strangers, an inn and hospital (Donahue, 1985, p.57). The core activities of nursing changed little through the centuries although the technical work of nursing altered with developments in science, knowledge and technology.

The beliefs, mores and culture of each civilisation directly influenced the way in which nursing care was given. Who rendered nursing care and what constituted nursing care were reflections of the prevailing society (Donahue, 1985, p.38). 
The social standing or 'place' of nursing altered in response to social need and expectation. Nurses were respected at times and vilified at others. Nurses worked in hospitals, homes, with communities in times of peace and war, in times of devastation and supply. At all times nursing has worked to support the common purpose.

To give jointly, to supply ideas or opinions, to be partly responsible for, to help bring about (something). These elements imply a reason or purpose to be jointly involved, a purpose to contribute, a mandate and authority to take action and be partly responsible for something. This is an important element of the meaning because it establishes the social mandate of nursing, the reason to be present that cannot be challenged.

Having a purpose to supply ideas, opinions or action is a strong element in the definitions of contribution. Having a purpose relates to the concept of social contract that was first discussed by the philosopher Rousseau. Ripstein (1999, p.222) explains these philosophical ideas as the purpose or a social bond that is formed "under a single motive and acting in concert", where there are several interests in common, a shared bond. The social community is willing because of need to form a bond with another community for a purpose, in this instance nursing. Nursing is motivated to form a social contract because individuals are willing to relinquish individual particular will and self-interest to embrace a 'general will', covenant or contract, that will benefit the group because to do so will allow protection. In the creation of a 'community' there is a certain kind of freedom "because it makes new 
modes of interaction possible by providing means for forming reliable expectations about one's fellows" to achieve a shared end (Ripstein, 1999, p.229). 'Shared ends' are important because "by forming an aggregation of powers strong enough to overcome any resistance, putting themselves under a single motive and acting in concert" (Ripstein, 1999, p.223) nursing as a collective achieves protection in exchange for acknowledgement of a valued position protected from challenge.

The philosophy of Rousseau has relevance for the involvement of nursing as a profession in common effort and assuming responsibility for aspects of the common effort. The concept of social contract and compliance with the 'general will' reflects the social responsibility that nursing as a profession espouses in statements such as: "Nursing has a social contract with society and so is a medium whereby social policy is enacted" (New Zealand Nurses Organisation, 1998). Nursing appears to accept the expectations that flow from the perceived social contract. Nursing is afforded self-regulation in exchange for consistent maintenance of standards and commitment to providing service for the goodwill of the community. This responsibility is acknowledged by statements such as:

nursing has a responsibility to society to provide a written statement clarifying the extent of nursing's missions, goals, relationships, responsibilities and accountability, thus establishing nursing's professional identity with the public (Goreberg, Alderman \& Cruise, 1991, p.11). 
For nursing to work towards a common goal, putting aside individual aspirations for autonomy or "natural independence" (Ripstein, 1999, p.229), there must be a trade-off. The profession of nursing, as did other professions, sought formal protection through legislation, of their place in service delivery, for their exclusive use of the title and accepted functions (Neill, 1961). The passing of legislation in 1901, the Nurses Registration Act 1901, allowed nurses to be licensed and to self-regulate. The achievement of legislation to provide protection developed a "we-intention" that set the conditions of unspecified future interactions and included a further condition of mutual enforcement. The expectation implied that an individual as a member of the community would do ' $\mathrm{x}$ ', that others would be held to do ' $\mathrm{x}$ ' and that others of the community would hold each other accountable for ' $x$ ' (Ripstein, 1999, p.223).

Reliance on legislation as the indicator of a formally negotiated social contract may appear tenuous, but it is generally accepted that while the members of the community must agree about their interpretation of what is meant by the social contract, formal agreement need not be overly explicit. The members of the community are understood to share a general will and agree in their judgements about what counts as violation of the general will, even if they cannot fully articulate any principles underlying those judgements (Ripstein, 1999, p.224). A social contract is an agreement in which all parties consent to the specified arrangements, all members of the community are a party to the contact and are thereafter socialised to share in the contract and the obligations (Ripstein, 1999, p.233). The powers and 
privileges allowed of the profession are conditional on their performance of their responsibilities (Morris, 1999, p.ix) and are removed if not adhered to. Nursing has stated its acknowledgement of a social contact and agreement to work through the Nursing Council of New Zealand to ensure understanding and compliance with the expectations of the wider society. Nursing as a profession demonstrates the seriousness with which it assumes responsibility for its actions by the stringency with which it manages entry to the profession and the penalties afforded those not complying with expectations (Nursing Council of New Zealand, 1999).

The authority 'of being partly responsible for' and undertaking certain specific nursing actions may not have been formally negotiated. It has been accepted over time, however, that a mandate for the scope of practice was given in the past century by awarding protection under the Nurses Registration Act 1901. This ongoing relationship has been reinforced each time the occupational legislation has been revised. Nurses have interpreted this to mean that they had exclusive responsibility for many actions and that if they delegate these they are held accountable for their decision making. This has been reinforced by the regulatory decisions of the Nursing Council of New Zealand to discipline or remove practitioners from practice. The law has similarly held nurses accountable for their actions, omissions and inaction.

The expectations of the profession by society have remained constant, although the activities of the profession to meet these expectations will have 
changed over time in response to the different social expectations, professional developments and technological advancements. The actions of nursing 'to bring about something' have remained aligned to a common effort to which the community was committed, for example, the support of isolated communities or the presence of nurses in war zones in the time of war. While formal contracts may alter because of political and managerial ideology, it is accepted by the social contract theorists that "to authorise someone to act in one's name is to appoint her as one's agent" (Gauthier, 1999, p.68). Nursing as a profession therefore is authorised to undertake actions to prevent illness, maintain health, restore health or support until death. The profession has worked as a social agent with individuals and in the communities of New Zealand, authorised to be called a nurse and to practice according to accepted scopes of practice. The place and mandate of nursing 'to act and give jointly' has evolved through the decades, reinforced by the respect and standing of practitioners and by the quality of the contribution of ideas and opinions, underpinned by the defined body of knowledge.

The absence in New Zealand of a detailed social contract or prescriptive legislation, as exists in law in some states in the United States of America, has had both a positive and negative impact on the profession of nursing. Positively it has not constrained the development of the profession through pre-established limits to the scope of practice. This 'permissiveness' has allowed the profession to change in response to socio-political environmental changes without requiring re-negotiation of legal boundaries. In a negative 
sense, the lack of formal negotiation has allowed the profession to develop without specifying its uniqueness, without developing a shared understanding with society of the expectations between the community and the profession. It has not required that the profession reflect on or evaluate its achievements. Instead, the profession of nursing has adapted and diversified, as it perceived was required in response to the health service demands, professional educational requirements and economic 'drivers'.

The changes to the actions of nursing have not always been understood by sections of the community, who have found in the main found the changes unsettling. Consumers generally retain a traditional view of what nursing is and how it should be provided and can be confused by changes that do not match their perception but reflect international models or management ideology.

While the concept of 'social mandate' implies accountability and partnership, nursing has not always recognised its responsibility to re-negotiate or to communicate with the community it serves about ongoing changes in practice and priorities. Some of these changes, such as academic degree preparation for entry to the profession, have occurred in response to external influences on the profession. The changes have been implemented with minimal strategic conversation with key stakeholders and has had the impact of excluding potential practitioners not able to reach the academic entry criteria, such as Maori and Pacific people. The rationale for change has not been clearly communicated to society with the result that they have not 
always understood what to expect from the changing face of nursing. There has been minimal explanation of why actions or activities undertaken previously have altered and there is little description of the benefits that can be expected. While nurses have adapted to changing service organisation expectations and adjusted to new technologies, local communities have not always understood the significance of these adaptations and have expressed disappointment when some of the basic care practices are no longer provided by nurses.

To give jointly reflects the work of nursing, since nursing works collaboratively within a multi-disciplinary team, co-ordinating the efforts of individuals, teams or services, helping to bring about action towards a common effort. To give jointly implies that there is equality and respect in the shared action, that what is offered is valued and accepted in a partnership of ideas. The right to contribute in an equal way acknowledges that the ideas and opinions have merit. Respect was achieved in the $1800 \mathrm{~s}$ with improvements in standards of practice and education of nurses. Nursing was acknowledged and supported and nurses opinion and ideas were sought after. This indicated a significant improvement in the status of nursing. The leaders of nursing in the early 1900s capitalised on this change in status by further progressing the place of nursing by emphasising further education, organisation and discipline. They made these changes based on social expectation and kept the community informed through detailed annual reports in response to social interest. In contemporary practice the views of 
nursing are less sought after and nurses are learning to use the media, internet and politicians to communicate in issues of socio-political interest.

To bring about a result, an act of contributing. Contemporary nurses, when asked to describe the purpose of their work, use descriptors about actions and tasks. In my experience they rarely describe their practice in relation to individual achievements or identified outcomes that reflect the result of an action taken. This may be because, despite the promotion of the nursing process some decades ago, nurses do not appear to routinely think about their practice as a systematic assessment of need, planning of inputs, action or intervention and evaluation or review of achievements. A number of nurses describe their practice as a composite of actions that are enacted in response to a sequence of needs or requirements. They are not specific about describing the professional skill such as 'assessment and monitoring of patient response to treatment' or 'care co-ordination through the episode of care'. The focus on routine tasks fails to communicate practice as a skilled systematic response to identified needs. It creates the perception of erratic action rather than sophisticated linking of assessment to planned action to achieve anticipated outcomes. The almost complete absence of evaluation and failure to acknowledge the result or achievement has resulted in the profession and the community having no way to acknowledge the skill of the practitioner in achieving the anticipated outcome.

Nursing has also been slow to claim the co-ordination role in the health care team as a way of bringing about a result. It is not clear to the community that 
Nursing is the essential co-ordinator in the health team that brings together the actions and plans of various health team members to address the identified needs and to ensure that the outputs are achieved. The integrating, co-ordinating and managing actions of the nurse are essential to the achievement of the overall goals of the whole health team, yet this function is not well understood or articulated as an essential activity to bring about a result, an act of contributing.

To bring about a result in a successful way requires flexibility and ability to use comprehensive expertise in a responsive manner. Nursing has demonstrated flexibility and responsiveness over time, as will be discussed in subsequent chapters. The reality is that in being flexible to bring about action to meet changing expectations, nursing has diversified to the point that nursing identity has become less distinct. Nursing does not have a universally consistent language or systematic way of ordering or documenting ideas so that its actions, achievements, diversity and responsiveness are explicitly stated so that they can be acknowledged, communicated and celebrated. Nurses do not document effectively how they demonstrate responsiveness to the needs of the situation through timely action once a need has been identified. This may be because of workload, but may also be because they do not identify this as a significant contribution.

Nurses are generally acknowledged as skilled practitioners yet the comprehensive knowledge required to be responsive is not well appreciated. This is of particular concern where nurses promote self-care that is then 
viewed as so easy even families can do it. What is not valued is the skill of breaking down the complex into achievable actions, coaching, motivating and recognising 'readiness to learn'.

\section{To supply ideas or opinions, to be partly responsible for, to aid a common}

goal implies that the individual or group has knowledge and expertise that is reliable and respected. It implies that what is offered is responsive to a need, a situation or circumstance. To supply ideas or opinions, the knowledge must have validity, be acknowledged as being of value and be experienced as trustworthy. This is particularly important in the delivery of health care and in work with 'at risk' communities.

In the 1860s, Florence Nightingale provided a role model for supply of ideas and opinion in response to obvious health problems. Her initial writing about conditions in the Crimea in 1854 and other writing relating to epidemiology, raised expectation of politicians and planners that leaders of nursing would have a strong knowledge base, would have an opinion and be able to assume responsibility for implementing ideas. Grace Neill (Neill, 1961), as Inspector of Factories in the 1890s, Inspector of Hospitals and then the first chief nurse in the Department of Health, similarly was a person to whom politicians and planners went for advice and guidance. The nurse pioneers were strong, opinionated, educated and capable of responding to most eventualities. The knowledge and skill required of nurses was emphasised as being comprehensive to cope with a variety of clinical settings, and people of different ages, gender and health needs. For the early 'backblock' nurses in 
1908, those establishing fever camps, or working in major post-war rehabilitation centres, the need for a broad, practical knowledge was essential as the nurses worked with minimal resources, to meet complex and variable demands. Nurses were also required to provide comment and opinion on many topics, were influential in advising government officials about the needs of local communities and achieved changes in services available to people in need (Chick \& Rodgers, 1997).

Over the past thirty years, however, nursing has become more aligned with medical specialisation (Lawler, 1999; Litchfield, 1998; Scott, 1998; Whyte, 2000) with emphasis on medical specialist supervision and techniques handed from doctor to nurse. As a result, many nurses describe the boundaries of nursing in terms of specialities and specific techniques or tasks rather than the autonomy of directing their own practice. In contemporary practice, nurses generally work in a single clinical speciality setting, and articulate their function in relation to specific tasks or activities. Care practices and activities are generally dis-integrated into single disease processes, such as haematology, or into single contexts, such as intensive care or haemodialysis, rather than focusing on diverse general care needs and the whole of the care process. This has given rise to concern about deskilling of the generalist with the advent of specialisation (Marshall \& Luffingham, 1998). The diversity of practice, the specialisation versus generalisation debate and the increased medical power because of specialisation, has resulted in contemporary nurses remaining involved as 'responsible for' co-ordinating the patient care plan and health team input, 
but perceived as doing so under direction of the specialist doctor. It is the medical practitioner who is commonly asked to comment rather than the nurse.

\section{Common themes identified as applying to nursing}

Throughout the discussion of the meaning of contribution and its application and relevance to nursing, a number of words and themes became obvious. There was a constant theme about expectations, where it could reasonably be expected that the contribution is a quality contribution, there is willingness to contribute as part of a team, to achieve what is expected and has been agreed, to commit to a shared vision and a common endeavour. There is a theme about action, an expectation about the willingness to take action and supply what is expected, to complete the action consistently and to an expected standard. There is a theme about achievement, where those receiving the contribution have an expectation that there is a result, achievement of the common purpose or aim, that the outcome is achieved as expected. There is finally a theme about responsiveness, where there is an expectation that there is flexibility and adaptability in the contributor so that there is give and take, ability to alter the act of giving and helping in response to altered aim or purpose.

Working with these themes of expectations, actions, achievements and responsiveness I was reminded of general systems theory of input, process and outcome with a feedback loop. The themes were inter-linked components, reliant one on the other to provide meaning and explanation. The systematic inter-connectedness also reminded me of the structure of the 
'Nursing Process' (Yura \& Walsh, 1988), where there was a four-part focus on assessment, planning, implementation and evaluation. Some essential elements also seemed to logically fall under the four main components to provide further explanation of the relationships of the components. These elements included: the nature of the service provided (purpose); goal or outcome; rationale for services; characteristics of the caregiver; characteristics of the recipient and the context for service. Explanations of the relationships of these concepts provided the framework for considering expectations (Meleis, 1991, p.460).

Using these ideas I then explored how a model might be constructed using Systems Science and Cybernetic Systems Theory, as these theories provided complementary explanations that related to the four main elements of expectation, action, achievements and responsiveness.

\section{Theoretical underpinning of the model}

While Systems Science and Cybernetic Systems Theory appeared to most closely apply to the construction of the themes arising from the interpretation exercise, I initially considered social constructivism as a theoretical base. This philosophy suggests that "the terms by which the world is understood are social artefacts, products of historically situated interchanges among people"(Denzin \& Lincoln, 1994, p.127). "Knowledge in some areas is the product of our social practices and institutions or of the interactions and negotiations between relevant social groups" (Audi, 1999, p.855). It seemed that the work of nursing as a social agent between relevant social groups 
reflected the reality of nursing endeavour. This approach appeared initially consistent with a "thought experiment" that sought to interpret and view nursing as a product of social practices, responding to community need and the influences of historical period. It was rejected, however, as the work was not to seek to understand the actions, reactions and perceptions of human beings. Rather, the purpose of the thesis was to find a model to assist nurses articulate their contribution and a sharper focus was needed.

The concepts of systems science and cybernetic systems theory more closely reflected the systematic components arising from the interpretation of the word 'contribution' and the reality of nursing responsiveness as a sub-system of the wider health systems, which is in turn a sub-system of society. Systems theory components then provide a parallel to the major units identified in the 'contribution model'. System Science theory appears relevant where a system is defined as

(a system in this context) anything that takes its integrity and form from the ongoing interaction of its parts... defined by the fact that their elements have a common purpose and behave in common ways, precisely because they are interrelated toward that purpose (Senge, Kleiner, Roberts, Ross, Roth \& Smith, 1999, p.137).

The inter-relatedness and action to support common ways to achieve a purpose resonated with the main elements in the definition about contribution but also the way in which nursing exists and relates within society.

\section{Systems Science and Cybernetic Systems Theory}


Systems Science and Cybernetic Systems Theory are relevant to the development of the 'Contribution Model' because these theories position the sub-system of nursing within the functional whole social system to generate a level of organisation that is fundamentally different from focusing on nursing as an individual sub-system. The theories also helps to explain the place of nursing as a complex sub-system within wider complex systems and the evolutionary processes of development that has allowed nursing to maintain, adapt, self-organise and remain oriented towards a future state (teleonomic behaviour).

The language of Systems Science and Cybernetic Systems Theory is generally well understood and is relatively simple to use. Systems theory is part of existing learning programmes for nurses as they are introduced to systems thinking, the influences of systems on the lives of the communities they work with and the influence of systems on their own place as a profession responding to wider systems. The work of many nurse theorists is based on systems thinking, including Martha Rogers in the Unitary Human Being theory; Dorothy Johnson in the Behavioural System model; Betty Neuman in the Systems Model; and Imogene King in the Systems Framework and Theory of Goal Attainment (Tomey \& Alligood, 1998). Nurses are introduced to these theories in their undergraduate and postgraduate study. The study of Systems Science is also by definition concerned with change, which is a constant feature of nursing as a sub-system of society in New Zealand. And finally another reason for using Systems Science is that systems can be modelled or created to theoretically replicate the behaviour of 
the original. This provides a way of applying the 'Contribution Model', using retrospective case study descriptions and futures scenarios. Systems Science provides a scientific approach to identify common structural, behavioural and developmental features in order to build a general theoretical model relevant to the phenomena such as growth, homeostasis and evolution.

For nursing action and achievements to be effective there is a need for a clear framework that ties everything together. There is a need for a model that assists the profession: understand society, the world and our place in it; to assist us make the critical decisions that will shape our future such as how to ensure growth, how to maintain homeostasis and how to evolve appropriately in response to social change and expectations (Heylighen, 1996).

\section{Key facts about systems and their relevance to nursing}

In order to show the relevance of Systems Science to nursing, the following section outlines the main components of systems and suggests linkages with nursing.

Systems Science focuses on the arrangement, interdependence and relationships of structures rather than the constant attributes of an object. Systems Science studies the regularly interacting and interdependent group of items that form a unified whole and the interdependence and interlinking of various subsystems within a given system that connect them into a whole. A system is identified as a functional whole composed of a set of subsystems and components that when coupled together generate a level of organisation that is fundamentally different from the level of each individual subsystem. 
A system can be defined as the understanding of the relationship between things that interact. Real systems are said to be open to and interact with their environments and continually evolve through acquiring qualitatively new properties in order to seek equilibrium, so the study of systems is by definition concerned with change. Nursing can be viewed as an open system, continually seeking equilibrium, as a system of its own but when coupled with other subsystems contribute to a fundamentally different level of system.

Systems Science theorists have described several common characteristics of systems that have relevance to this discussion. These include the systemenvironment boundary, inputs, outputs, processes, a state of being, hierarchy, goal-directedness and information. Open systems are described as importing energy from the environment as inputs. The imported energy is then transformed into some product form or pattern. The output or exporting of that product into the environment occurs as matter, energy, information and services is part of the output function. Open systems also share the characteristics of negative entropy, feedback, homeostasis, differentiation and equifinality. An open system develops a cyclic pattern of activities reinforced by the necessary inputs and an exchange of outputs or activities with the environment. In order to survive and maintain their characteristic internal order, the system must import from the environment more energy than they expend in the process of transformation and exportation. Selected information serves as feedback signals to an open system concerning its functioning and the nature of its environment. Negative feedback helps a 
system to regulate its functioning as conditions change in its operation and environment. Coding translates selected information into meaning.

An open system that survives must maintain a balance in the exchange of matter and energy with the available information and acquire control over external forces. This steady state ensures a dynamic equilibrium. An open system tends to develop highly specialised subsystems or differentiation in order to perform system functions. A system may reach a state of equifinality to achieve a given outcome or result. Nursing as a subsystem is sensitive to the system-environment boundary, uses information as inputs and produces multiple processes to create outputs, is responsive to positive and negative feedback, and is generally goal-directed as a sub-system. While the subsystem seeks to maintain homeostasis, there is at times some difficulty with self-organisation. Based on positive feedback the result may be an explosion or blocking rather than maintenance of equilibrium and convergence.

Systems require inputs that allow for entry of information, resources and stimuli, the environment's influence on the system. In the 'Contribution Model' the inputs include clarity about the expectations of various stakeholder groups and the professionals through which these expectations will be transformed into appropriate action to ensure that achievements or system outputs can result. Input and output are separated by a duration of time while action occurs, as in before and after or past and present. In every feedback loop, information about the result of a transformation or an action is sent back to the input of the system in the form of input data. If this new data facilitates and accelerates the transformation in the same direction as the 
preceding results then they are considered positive feedback and their effects are cumulative, leading to divergent behaviour of exponential growth or total blocking of activities leading to decline which will destroy the system. If the new data produces a result in the opposite direction to previous results they are negative feedback and their effects stabilise the system and maintain the equilibrium. This leads to adaptive or goal-seeking behaviour, to sustain the same level of oscillation around an ideal equilibrium that it never attains (de Rosnay, 1997).

Systems have processes that focus on maintenance and self-organisation. Self-organisation is a basic process of evolution where the effect of the environment is minimal and where new, complex structures develop in and through the system itself, triggered by internal variation processes, called "fluctuations" or "noise". After the self-organisation process has occurred, there is less ambiguity about the state of the system. There is a decrease in thermodynamical entropy because the system exports or efficiently dissipates the heat or entropy generated to sustain it, into its surroundings. The system therefore has the capacity of changing to higher levels of orderliness.

Systems contain subsystems that continuously fluctuate. A single fluctuation or combination may be magnified so that the system shatters, as a dissipative structure, to the pre-existing organisation. For example, changes in the wider social system, such as economic instability, impacts on health as a subsystem. When government cuts the revenue to main health providers this then has an impact on nursing programmes. Health system restructuring has an impact on nursing and on community access to services. Cybernetics then 
studies the circular feedback mechanisms that allow complex systems to maintain, adapt and self-organise towards a future state (teleonomic). In contrast to linearity, which means that effects are proportional to causes, cybernetics suggests that telenomy and causality can be explained using nonlinear, circular mechanisms where the cause equals the effect. Using this application, negative feedback for self-maintenance supports homeostasis and non-linear interaction between the goal-directed system and its environment results in control of the system over the perturbations from the environment (Heylighen, 1997, b).

This deterministic principle of chaos is important in acknowledging that chaotic processes work as amplifiers, turning small causes into large effects. Small unobservable fluctuations will affect the outcome of the process; causes that seem equal to the best of our knowledge can still have unobservable differences and therefore lead to very different effects (Heylighen, 1998). This is relevant for the discussion on nursing contribution because the ability of nursing to deliver on the expectations and therefore achieve the outcomes can be significantly affected by small fluctuations beyond the control of the sub-system of nursing. The opening of a new hospital in a community and recruitment of staff for service delivery can destabilise the workforce supply so that existing services in the region cannot be maintained. Nursing has demonstrated organised processes to maintain goal-directedness despite perturbations from the environment. Unpredictable change and growing complexity however has impacted on the ability of the profession to adapt to information overload and knowledge fragmentation. 
Small unobservable fluctuations in political alliances have affected the outcome of the process. Subtle change in causes, such as election of new governments, can result in differences in input-expectations therefore leading to very different effects.

A system is considered more complex if more parts can be distinguished and if more connections exist between them. Complexity can be characterised by lack of symmetry. Complexity increases when the variety (distinction) and dependency (connection) of parts or aspects increase in several dimensions. Increase of variety is called differentiation and an increase in the number or strength of connections is called integration (Heylighen, 1997, a). For the profession of nursing, as more has been required in terms of diversity and in scope of practice, the more complex the system inter-relationships have become, increasing the lack of symmetry. Increasing complexity is evident in service specialisation, increasing integration between primary and secondary referrers and services, between secondary and tertiary relationships and increased range of services.

Another relevant concept of System Science is that of selection, or the reduction in the quantity of variety in the process of selection, a limitation of the number of possibilities. Also included in the concept of selection are forms of stability and equilibrium. Selection can be internal when an unstable system spontaneously ceases a process, or external when a system is eliminated because it is not adapted to its environment. Fitness is a measure of the likelihood that a configuration will be selected (Heylighen \& Joslyn, 1996). Decisions made internally by the profession as a subsystem have had 
significant impact on the stability and equilibrium of relationships within the system and sub-system. Externally the demand from society for nursing to change processes or be subject to challenge of 'fitness' is a current issue in certain service specialities.

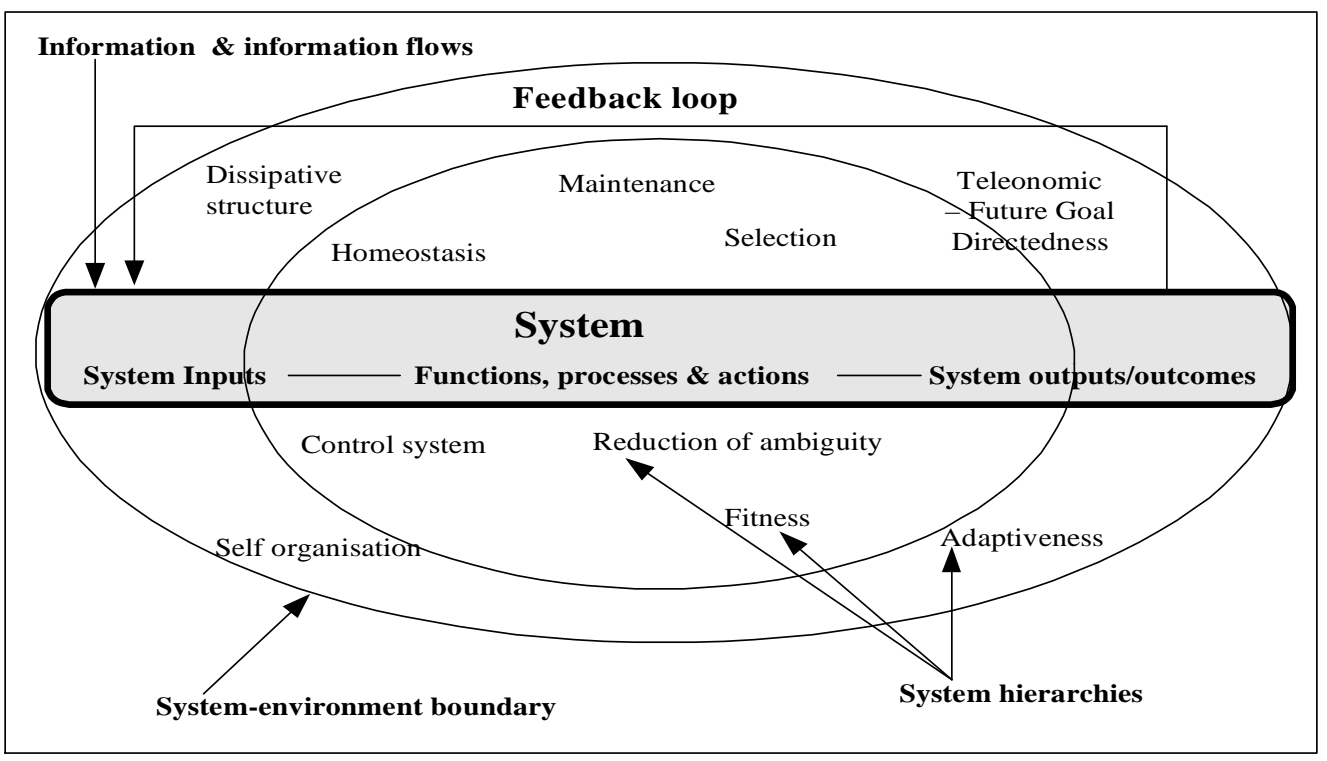

\section{Figure 1. Complex adaptive systems in a system-environment}

Cybernetic ontology refers to the ontology of action. Action as part of a system is a result of a free choice. This is relevant because nursing reserves the right to decide for itself the 'what' and 'how' of its actions to meet the expressed expectations or identified needs. Action is a key feature of the meaning of the word 'contribution' and the ability therefore for the profession to demonstrate relevant action is critical. The 'state of the world' is described, as a set of feasible actions for each will. The 'act of will' is to choose one of these feasible actions learned through our interactions or representations with the external world. For a system to function, 'an agent' is identified as a representation of an action, the 'carrier of will', and the 
entity that performs the action and chooses between possible actions. The 'agent' is not always seen but what they do is seen through the interdependent actions of communication, control and information. Actions can take place in parallel and others consecutively. Action has ontological primacy and a definite reality. A process is a conceptual scheme: a process is an action that is a sequence constituting sub-actions, a sequence of its stages. A process has a definite initial stage, the input, and an abstraction from the final stage, the output. The process transforms the input into the output and represents an ongoing change. Actions form a significant characteristic of the culture and practice of nursing. Nursing is identified in many situations as the 'agent' of change. Nursing is given authority to chose the actions that are appropriate to deliver the achievement (outputs and outcomes).

The following diagram depicts the flow of action through the agent and the influence of this on the inputs and expectations that arise from the feedback loop.

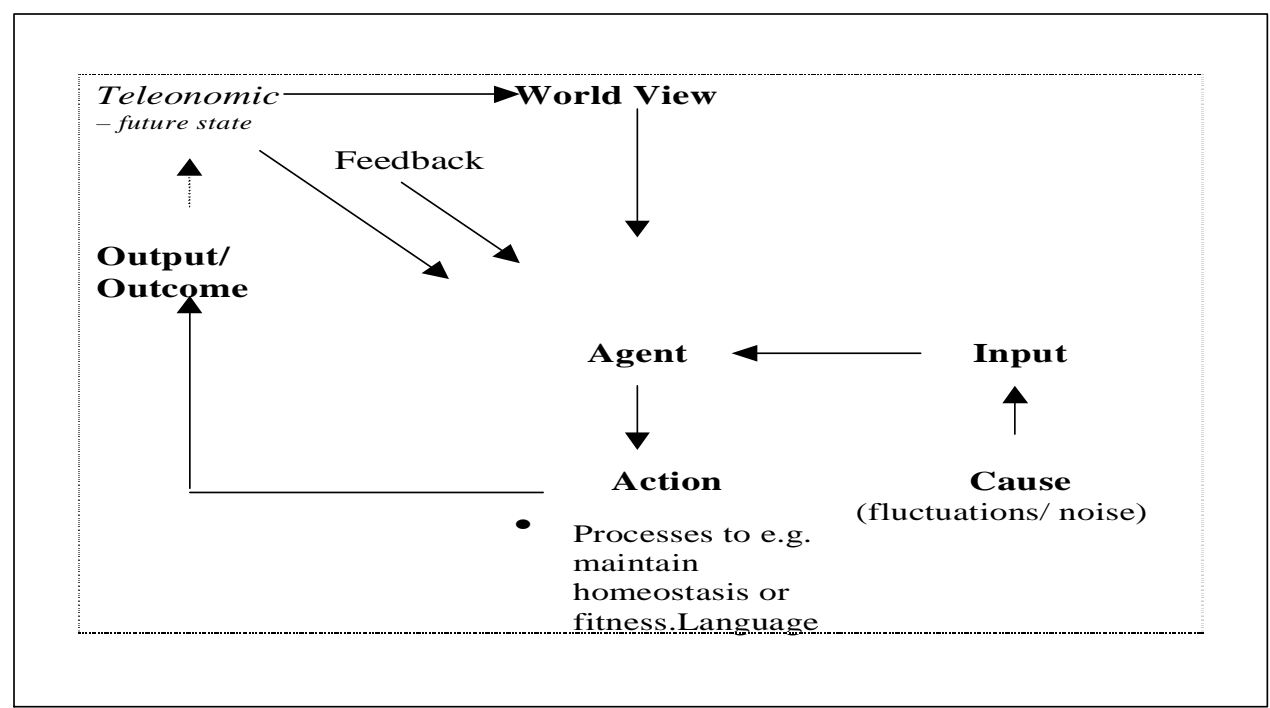

Figure 2. Diagram depicting flow of influence on the agent 
Nursing as an agent undertakes appropriate actions that transform into outputs. For the professional, the 'how' of practice is the choice of a range of potential actions that can be used to select the best outcome on behalf of their patients. Professionals seek a practice environment where there is autonomy to choose the right action, to use their knowledge and experience, as an action of free choice (Shaw, 1986; Clifford, 1999).

In systems science theory, messages communicated as language, called commands and statements, provide feedback to influence the actions performed by the agent. There are two ways of changing the actions of an agent: one is to change the 'state of the world' as far as the agent is concerned and the other way is to change the agents 'model of the world'. Changing the agents 'model of the world' can be achieved through the formalised embedding of a concept or concepts in language that depend on the 'form' of the objects and not their human meaning. The 'form' is the material carrier of the concept that can be examined and manipulated as an objective object. The 'form', as a communicated concept, can then be considered theoretically, as the 'model of the world' is presented as a conceptual framework to be used in construction of formal theory (Turchin, 1997). Knowledge can then be communicated about a complex system, as depicted as a model of some part of reality. In this instance the 'form', as carrier of a concept namely contribution, is modelled so that it can be examined and manipulated. The 'Contribution Model' provides a way of linking the systems science ideas and the main themes identified in the word interpretation phase to show how the ideas could be applied to practice. The 
'Contribution Model' can benefit the profession, by assisting to change the professions 'model of the world'. The case study method provides a threedimensional perspective of a situation by gathering information about what is happening in society, what is happening in health and then what the nursing response is to the situation. The 'Contribution Model' guides the user to understand the expectations, to match the most appropriate actions to achieve the outcomes expected. Using this methodology and language that is different from everyday meaning, yet familiar in concept, the model of the world can be altered to more effectively achieve the expectations.

In the end, the value of a model such as the 'contribution model' to nursing as a profession and nurses as practitioners, will be how the framework is used according to criteria such as: social significance, in encouraging the clients to be asked whether the service of nursing was significant to their health; social congruence where the clients evaluate whether the service of nursing was congruent with their expectations; and social utility where the usefulness of the conceptual model is evaluated as providing useful direction to education, practice and research (Johnson, 1974).

In this chapter the concepts arising from interpretation of the various meanings of the word contribution and the relevant major units from the general systems theory have been explored. These form the theoretical foundation for the 'Contribution model'. In the next chapter, the model is developed, based on the concepts from this chapter, to explain the framework 
and conceptual model that is presented to assist nurse's articulate nursing contribution. 


\section{Chapter Three}

\section{Contribution as a conceptual model}

"Nurses who have a clear, concise conceptual base specific to nursing will be able to...demonstrate in their own practice nursing's contribution to health care" (Harbour, Creekmur, DeFelice, Doub, Hodel, Tomey, Petty, 1998, p517). As discussed previously, many nurses are not able to articulate their contribution. The challenge then is to seek a concise conceptual framework that can assist them to articulate of their practice. This is the purpose of this theoretical work.

Despite decades of academic development in the profession, there is no single conceptual model published that clearly articulates the concept of contribution. The range of theories proposed by nurse theorists over the past fifty years have provided many nurses with a philosophy and model of practice but the emphasis is on process and not outcomes. The utility of the available theories has been variably encouraged in Schools of Nursing with the result that the theories and their application is not clearly evident in any practice setting in New Zealand, although attempts have been made at times. As a result nurses, in many health care settings, lack a mental image of nursing and this compounds their inability to communicate clearly and explicitly about the service they offer to society.

In this chapter the conceptual model of articulating contribution will be developed in more detail and an example demonstrated of how nurses might 
use the framework. In the previous chapter, the reason for focussing on the concept of contribution was explained and its applicability to the world of nursing outlined. The theoretical source, namely the general systems theory, was also explained. In this chapter, the assumptions, values and major units will be drawn together to create a framework that can be used in different contexts to communicate about the contribution of the general work of nursing and the impact of specific roles.

\section{A conceptual model}

A conceptual model for a discipline "is a very broad perspective, a mental representation or conception of nursing that is sufficiently complete and explicit so as to provide direction for fields of activity. It is an abstraction, a way of looking at something, conceptualising a reality, an invention of the mind. It is a precursor to a theory, specifying the focus of inquiry, identifying the phenomena of interest and providing a broad perspective for research, practice and education" (Adam, 1980, p.116-118). "A conceptual model is made up of assumptions, values and major units" (Harbour, et al, 1998, p.521). The contribution model that follows meets these criteria. Using the concept of contribution, some interpretations and themes have been abstracted into a system of interrelated propositions that are used to describe, predict and explain the 'Contribution model'. The model that has evolved, creates a conceptual departure point that can be linked to the reality and context of nursing practice. 
On examining the concept of 'contribution' and the interpretations that arise from the meanings as they applied to nursing, a number of major units were identified that have been used in the 'Contribution model'. These units parallel the major concepts in the general systems theory of inputs, process and outcomes and form interrelated patterns.

The major units identified were 'expectations', 'actions', 'achievements' and 'responsiveness'. 'Expectations' were paralleled to inputs, since expectations in the form of information and stakeholder assumption were inputs that triggered action to be taken. The resulting actions taken, or practice interventions, reflected the professional response to the inputs or expectations received. The 'actions' or interventions taken resulted in 'achievements' or outputs of some kind. Depending on the 'outputs' achieved, there was an assumption of a feedback loop to provide evidence of stakeholder satisfaction with achievements. The feedback loop was depicted as responsiveness, which assumes continued interaction, adaptation and responsiveness. Being responsive, that is acting on the inputs to adapt actions or interventions, is essential for continued social significance and social congruence. Responsiveness as a feedback loop is the constant influence on all of the component parts. A subsystem in a complex wider system must be responsive to the expressed inputs and must be able to adapt actions accordingly.

These major units apply to nursing as a professional subsystem, because the 'world' or wider system has expectations and 'laws' about how the 
subsystems will interrelate to achieve the shared goal of service provision and achievement of health goals. There are accepted processes and anticipated actions that will occur in the subsystem to achieve the desired outputs. The outputs will be evident and observable even if the actions of the agent are not.

In order to communicate a model as a system, a formula is communicated that can be explained and used as a generator of prediction. Formally, a model is a system depicted as $\mathrm{S}=\langle\mathrm{W}, \mathrm{L}, \mathrm{M}, \mathrm{E}\rangle$. W is used to describe a modelled system or the world. $\mathrm{W}=\langle\mathrm{W}, \mathrm{L}\rangle$ with $\mathrm{L}$ being the behavioural actions or natural laws within that world. $\mathrm{M}=\langle\mathrm{M}, \mathrm{R}\rangle$, with $\mathrm{M}$ being a representation or interaction and $\mathrm{R}$ being a set of rules. $\mathrm{E}$ refers to a measurement, a perception or an observation. When the functions of $\mathrm{L}$ (behaviour), $\mathrm{R}$ (rules) and $\mathrm{E}$ (measurement) interchange then $\mathrm{S}$ is a good model. The modelling system $\mathrm{M}$ can predict the behaviour of the world and $\mathrm{S}$ can be seen as a generator of predictions. Abstraction is always used in the mapping process as some aspects of the 'state of the world' are necessarily ignored in order to move from reality to its description. Abstraction is important as it reduces the amount of information the system $\mathrm{S}$ has to process before decision making can occur (Turchin, 1997). In using this formula $S$ system refers to the sub-system of health. $\mathrm{W}=\langle\mathrm{W}, \mathrm{L}\rangle$ with $\mathrm{W}$ referring to the world of nursing and $\mathrm{L}$ referring to the actions taken by the profession in response to identified need as appropriate to professional knowledge at the time. $\mathrm{M}=<\mathrm{M}, \mathrm{R}>$, with $\mathrm{M}$ being an interaction that nursing has with society that forms the feedback loop and $\mathrm{R}$ being the rules about expectations that society has of nursing. E refers to measurement or perception or in the 
'Contribution Model' the expectations demonstrating the achievement of expectations measured as outputs or outcomes.

In addition to a formula, a model must have evident a number of key principles and laws that underpin the framework or methodology for problem solving. The principles have the following properties: primitive in that it is a beginning to a system of thought and something can follow from them; simplicity so as to be self-evident or tautological; and universality to have virtually universal applicability. The aim is to arrive at a network of concepts and principles similar to a formal system with axioms defining primitive concepts, definitions of higher order concepts and theorems derived from the more primitive axioms and definitions (Heylighen, 1992, p.4). In constructing a conceptual framework or model in a complex dynamic system, a number of other principles are also acknowledged: complexity (complex structures with many heterogeneous interacting components); mutuality (components interacting in parallel, co-operatively and in real time creating multiple simultaneous interactions among subsystems); complementarity (subsystems that participate in multiple processes and structures); evolvability (systems evolving and growing in an opportunistic manner rather than designed and planned in an optimal manner); constructivity (as the system increases in size and complexity simultaneously bind to previous states while developing new traits) and reflexivity (through both positive and negative feedback the system is able to initiate self-reference, self-modelling, self-production). In the construction of the Contribution Model that follows these principles will be evident. 
The 'Contribution Model' developed and applied in this thesis is a unified and sequenced design of concepts and principles that can be used to describe the arrangements of and relations between the parts to connect them into a whole (Heylighen \& Joslyn, 1992). A model or conceptual framework is said to be most effective when it is based on well established theory, that is, there is correspondence. It must also be clear what the model is supposed to do, that is, it is coherent. It must be simple and applicable with minimal chance of fault and be based on clear requirements so that it is consistently applied. These features will be evident in the description of the 'Contribution Model' that follows below.

In order to communicate a model as a system, a formula is communicated that can be explained and used as a generator of prediction. Formally, a model as a system was depicted as $\mathrm{S}=\langle\mathrm{W}, \mathrm{L}, \mathrm{M}, \mathrm{E}\rangle$. W was used to describe a modelled system or the world. $\mathrm{W}=\langle\mathrm{W}, \mathrm{L}\rangle$ with $\mathrm{L}$ being the behavioural actions or natural laws within that world. $\mathrm{M}=\langle\mathrm{M}, \mathrm{R}\rangle$, with $\mathrm{M}$ being a representation or interaction and $\mathrm{R}$ being a set of rules. E refers to a measurement, a perception or an observation. When the functions of $\mathrm{L}$ (behaviour), R (rules) and E (measurement) interchange then $\mathrm{S}$ is a good model. The modelling system $\mathrm{M}$ can predict the behaviour of the world and $\mathrm{S}$ can be seen as a generator of predictions. Abstraction is always used in the mapping process as some aspects of the 'state of the world' are necessarily ignored in order to move from reality to its description. Abstraction is important as it reduces the amount of information the system $\mathrm{S}$ has to process 
before decision making can occur (Turchin, 1997). In using this formula $S$ system refers to the sub-system of health. $\mathrm{W}=\langle\mathrm{W}, \mathrm{L}\rangle$ with $\mathrm{W}$ referring to the world of nursing and $\mathrm{L}$ referring to the actions taken by the profession in response to identified need as appropriate to professional knowledge at the time. $\mathrm{M}=\langle\mathrm{M}, \mathrm{R}>$, with $\mathrm{M}$ being an interaction that nursing has with society that forms the feedback loop and $\mathrm{R}$ being the rules about expectations that society has of nursing. E refers to measurement or perception or in the 'Contribution Model' the expectations demonstrating the achievement of expectations measured as outputs or outcomes.

In addition to a formula, a model must have evident a number of key principles and laws that underpin the framework or methodology for problem solving. The principles have the following properties: primitive in that it is a beginning to a system of thought and something can follow from them; simplicity so as to be self-evident or tautological; and universality to have virtually universal applicability. The aim is to arrive at a network of concepts and principles similar to a formal system with axioms defining primitive concepts, definitions of higher order concepts and theorems derived from the more primitive axioms and definitions (Heylighen, 1992, p.4). In constructing a conceptual framework or model in a complex dynamic system, a number of other principles are also acknowledged: complexity (complex structures with many heterogeneous interacting components); mutuality (components interacting in parallel, co-operatively and in real time creating multiple simultaneous interactions among subsystems); complementarity (subsystems that participate in multiple processes and structures); 
evolvability (systems evolving and growing in an opportunistic manner rather than designed and planned in an optimal manner); constructivity (as the system increases in size and complexity simultaneously bind to previous states while developing new traits) and reflexivity (through both positive and negative feedback the system is able to initiate self-reference, self-modelling, self-production). In the construction of the Contribution Model that follows these principles will be evident.

The 'Contribution Model' developed and applied in this thesis is a unified and sequenced design of concepts and principles that can be used to describe the arrangements of and relations between the parts to connect them into a whole (Heylighen \& Joslyn, 1992). A model or conceptual framework is said to be most effective when it is based on well established theory, that is, there is correspondence. It must also be clear what the model is supposed to do, that is, it is coherent. It must be simple and applicable with minimal chance of fault and be based on clear requirements so that it is consistently applied. These features will be evident in the description of the 'Contribution Model' that follows below.

To model the way general systems theory applies to nursing and the contribution model, the following formulae and criteria are used:

The system is $\mathrm{S}=\langle\mathrm{W}, \mathrm{L}, \mathrm{M}, \mathrm{E}\rangle$, with $\mathrm{W}$ (system or world), $\mathrm{L}$ (actions or natural laws), $\mathrm{M}$ (representations and rules), E (measurement, perception or observation). In the contribution model, W refers to the wider system or 'world' of health services in relation to society, with nursing as a subsystem 
of the wider system. The 'world' has expectations of how the subsystem will respond as an agent to the inputs from the internal and external environment. $\mathrm{L}$ refers to the actions undertaken by the agent to meet the identified needs of the system or 'world'. M refers to (M1) as the rules governing how the subsystems interact within the world in order to deliver the actions required and (M2) being representations and rules of how feedback is used to ensure that actions remain responsive to social need. E refers to perceptions with (E1) being what the 'world' expects of behaviour of the agent and (E2) how the achievements will be validated. The 'Contribution Model' is therefore depicted as $\mathrm{S}=<\mathrm{W}, \mathrm{E} 1 \mathrm{M} 1, \mathrm{~L}, \mathrm{E} 2, \mathrm{M} 2>$.

The contribution model comprises interrelating major units. The expectations of the system (society and health), stakeholders and the world of the agent (nursing), influence the actions, interventions and practices undertaken by the agent to meet the expectations. The actions taken which result in achievements that can be observed and validated are monitored through feedback loops to ensure that the subsystem is responsive and can change to enhance the possibility of movement and growth.

Using the formula and applying it to nursing, the 'Contribution Model' can be described as a 'good model'. L, R and E interrelate. L, as behavioural actions, are well defined in the speciality scopes of practice and practice standards that the profession uses to respond to expectations and identified need. R, as rules, could be identified as expectations against which professional contribution is evaluated. E, as measurement, is identified as 
'achievement' in the model as it includes a way of demonstrating how the expectations were met.

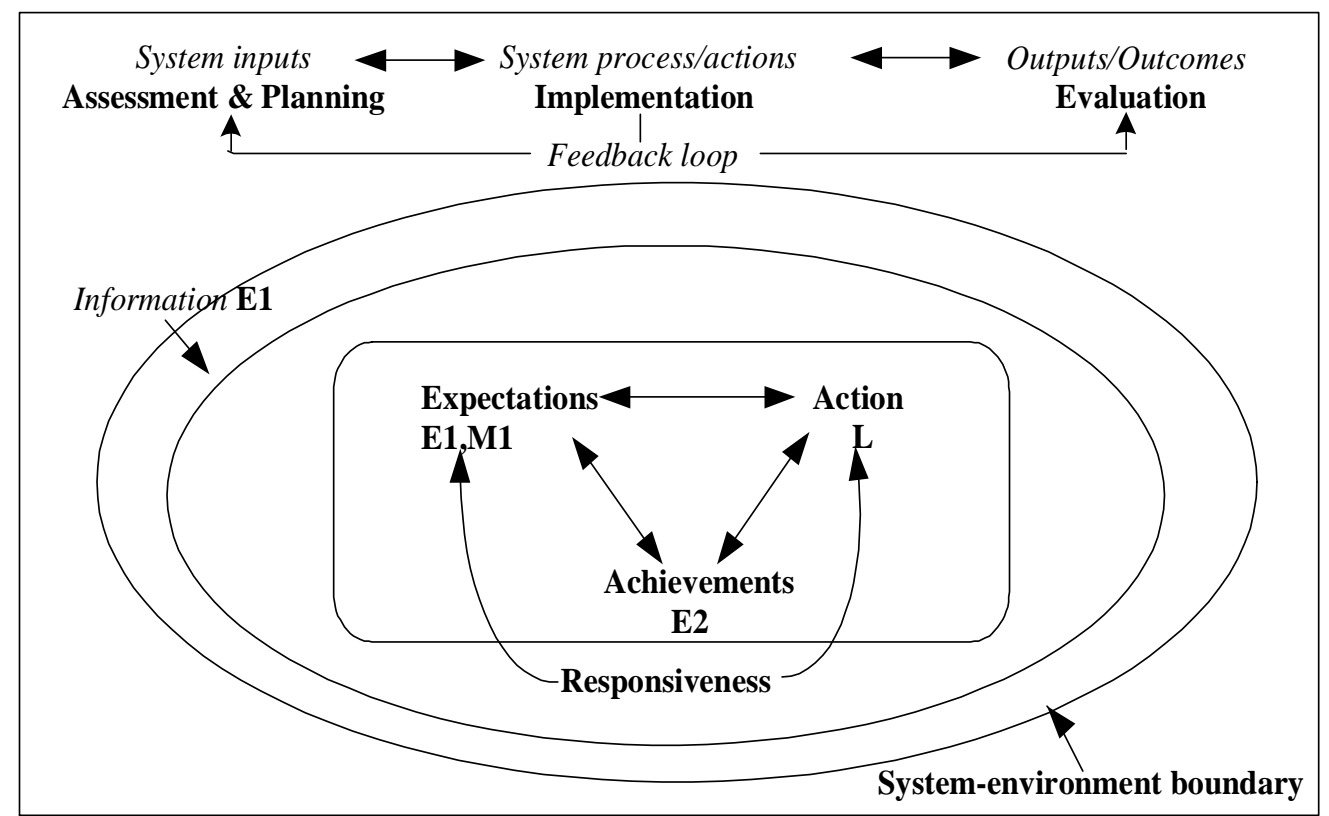

Figure 3. Complex adaptive system with nursing as a sun-system and the 'Contribution Model' depicted

The inter-relationships of the major units of the 'Contribution Model' can be depicted as in figure 3 below. The model can be simply described in a formula as 'Expectations' + 'Actions' $=$ 'Achievements' with Responsiveness as the feedback loop influencing changes to expectations and actions. There are further major units that define the model and its application. Essentially then, the model is simple and parallels the major units of the general systems theory.

The 'Contribution model' relies on the subsystem or agent understanding the expectations, including inputs of information both internal and external sources, such as language, commands and statements from various sources in society and the wider system. Understanding the expectations allows for 
matching of the actions and interventions to achieve the expectations, outputs or outcomes. Feedback is sought and inputted at all points along the continuum to ensure responsiveness to positive and negative feedback.

The inputs are communicated as Expectations, since information from internal and external sources clarifies the needs, demands and requirements of the various stakeholders. Nursing exists as a service to individuals and communities and their needs and expectations will in part direct the actions because nursing actions are planned in response to identified need. Nursing also exists in the context of social, scientific and professional expectations and must understand the expectations of these other stakeholders in order to remain credible and viable as a profession. Actions are identified in the process-transformation segment with a two-way link to expectations, as the input may direct actions but actions may also alter expectations. The Achievements component is linked to action because it is through action that achievements are made visible and therefore can be measured. Responsiveness is identified as the feedback loop. Responsiveness is an important element that monitors sensitivity to changed expectations and therefore influences change in actions in response to changed expectations. The elements are inter-related and sensitive to changes in the environment.

\section{Putting it all together: the model and its component parts}

Each major unit of the 'Contribution model' has elements that guide the user to consider what makes up the model. In the following section of this 
chapter, the elements in each of the major units will be explored in more depth.

\section{Expectation}

'Expectation' relates to the input component in the model. Expectation is defined as "the act or an instance of expecting or looking forward, something expected or hoped for" (The Concise Oxford Dictionary, 1990, p.411). The term expectation implies that there is an understanding of what is looked for and what is anticipated. This understanding may be communicated through general information, through formal negotiation in the form of a contract or may be agreed through an informal agreement or assumption of what is expected. The expectation may be explicit or implicit, may be stated or assumed that because of the professional education of the agent certain actions will be undertaken and need not be categorised.

The major unit of 'expectation' assumes that the following are understood: the goal of the profession; the beneficiary or the patient in this instance; the role of the nurse; the source of difficulty or reason for nurse-patient interaction; the anticipated intervention focus and modes are known; and the anticipated consequences or achievements are clarified. These elements are consistent with the major units of a conceptual model (Adam, 1980). The major unit of expectation seeks clarity in these elements, including (i) stakeholder expectations that might include the beneficiary and source of difficulty, differing expectations of the customer, employer, colleague and profession. In order to be clear whether the stakeholder expectations are realistic or not, it is essential that there is an understanding by the agent of 
(ii) the purpose and function of the work undertaken, so that this can be compared to the different stakeholder expectations. Agreement can then be reached about what the actual purpose of the role or function is or the goal of the profession. Its is important that there is acknowledgement of the (iii) skill and expertise available or required to achieve the expectations. This could be explained as the intervention focus and modes. Without clarity about this there may be mis-understanding of the degree of expertise required and might explain non-achievement because of lack of expertise or inappropriate delegation. Another important element is agreement about the (iv) anticipated outcomes, or consequences so that any variable expectations can be managed. The agent will need to be able to achieve what is expected, to put in place measures that will capture evidence of achievement so that there can be detailed and appropriate goal setting.

Describing expectations does not include describing the actions to be taken, although there must be alignment with expectations and actions for credibility and trust. The profession decides the 'what' and 'how' of professional practice actions appropriate to the agreed expectations and accepts the responsibility to direct its own practice (Shaw, 1986). The presentation by Shaw is reference because she provided a direct message to nursing at a crucial time, advising the profession to decide for itself the 'what' and 'how' of their practice, despite the advent of general management where nurses were managed by non-nurses. What is agreed is that the profession can decide how best to meet the expectations. 
Clarity of expectations in all the element areas is essential to ensure that the provider of service is able to negotiate to deliver expectations because there is clarity of purpose or function. Clarity of purpose and function allows for negotiation of alternative views where dissonance is identified. Knowing what knowledge and skill is required to deliver the purpose or function allows for planning of skill mix, differentiated practice or staff development so that the agent can deliver the expectations. It also ensures that the right people can be selected to undertake the function to achieve the anticipated outcomes. Having clarity in these areas enhances the chances of success for the agent and the stakeholder. It also allows for career development and education planning. In a formal contractual relationship it ensures that all parties have a clear understanding of the expected outcomes to be achieved and allows for appropriate resourcing, data gathering and matching of actions to expectations. Having clarity contributes to the probability of success.

Having clarity of expectation does not always mean success or that action will follow. To provide a service that meets all expectations means that some prioritisation must occur, skill must be matched to expectation and action selected to achieve the most effective outcome. The elements of 'Expectation' therefore include having explicit understanding of who has expectations and to whom or what the action will be directed, the purpose of the action, the knowledge and skill required to undertake the action and what the anticipated output or outcome will be. Without clarity of these elements there is potential for unfocused action, wasted time and energy on activities that fail to meet expectations and the potential for disappointment or missed opportunity. In summary the elements of 'Expectation' include - 
1 the characteristics, identity and expectations of the stakeholders who might be the customer/ 'client'/ recipient of the service.

2 the nature and context of the service provided and its purpose and function

3 the characteristics of the provider: skills and knowledge required of the caregiver

4 goal or outcome: anticipated outcomes.

The 'Expectation' is the first part of the model. It establishes what is required, for whom, to achieve what and who is best educated and skilled to provide what is required. Expectations may change over time and may be multiple and varied requiring some negotiation with the various stakeholders.

\section{Action}

'Action' is the process component of the model. It is defined as "the fact or process of doing or acting, forcefulness or energy as a characteristic, the exertion of energy or influence, something done, a deed or act, a series of events of the story" (The Concise Oxford Dictionary, 1990, p.12)

The component of 'Action' refers to the main activities of the work or role undertaken to achieve the 'expectations'. The actions are objective and observable, they are specific and contribute to the plan of care or intervention relevant to meet a need, situation or event. The actions or interventions can be linked to the assessment of a need, they are consistent so that it can be anticipated that a composite of actions will result in a particular output or 
outcome. The importance of relevant, validated and consistent action is essential to the effectiveness of the model because it reassures the stakeholder that their expectation is understood and is being worked on.

The 'action' component of the model is where nurses have traditionally found it easier to communicate what they do by describing tasks and actions or intervention focus or modes. While it may be easier, it has also been very difficult to get agreement on a common language and a way to classify the groups of activities. Nurses are increasingly recognising that they need to find a way to record their practice to give a more comprehensive and accurate picture of what they do, to demonstrate their accountability and effectiveness. Projects initiated in the 1980s to identify standardised language to describe the 'action' component of nursing practice have made slow progress.

In almost every country of the world nurses experience problems of powerlessness due to the invisibility of nursing in the information systems...Nurse managers and policy makers want to be able to compare nursing activities and performance across time and place and are increasingly frustrated by their inability to compare their results (Clark, 1998, p.3).

While in my experience nurses state that they find it easier to describe their practice actions, in reality this is erratic and incomplete. Nurses use different language or descriptions for different purposes. For example, the language describing clinical terms in practice and the definitional terms or the 
language of nursing knowledge is slightly different in different cultures, even between western countries (Meerabeau, Casey, \& Old, 1997). While in some respects standardised terminology and classification give a more comprehensive and accurate picture of what nurses do, this can be sterile. Individualised and context-specific descriptions can have more impact and allow for more culturally specific examples.

The 'actions' elements in the 'Contribution Model' alter with each clinical speciality or role, with time, resources, expectations and context. It is helpful to consider use of a taxonomy of interventions and outcomes rather than relying on existing practice of communication about nursing tasks. While it would be ideal if the significance of the 'action' element could be listed and fully appreciated by a stakeholder, this could over-emphasise the importance of actions and reinforce the power of the stakeholder to direct the 'what' and 'how' of nursing practice, rather than them just specifying expectations. It is important that the 'what' and 'how' of nursing, or the focus and modes, be directed by the nursing profession.

Stakeholders may identify certain actions that they wish to see happen, and it is important to listen because what they describe is often what they value. What the stakeholder expects to see may not technically be the highest priority or value to the health outcome, nevertheless it may be highly valued by the consumer and nurses need to not lose sight of the expectations and images that give relative comfort. There may also be dispute if the actions thought to be of value to the consumer are not visible to the stakeholder. The 
'actions' component of the model can be the most influenced by the perception of the stakeholder as they match the actions of the modern practitioner with the memories and images of nursing from the past which might be more myth than factual. For instance, in nursing the skill of assessment and use of all senses in undertaking this assessment is not always understood.

The public does indeed have a specific image of nurses. That image is shaped by the impressions people have gained of the attitudes and behaviour patterns of nurses, from observing them at the bedside of family members, and also by various stereotypes and social norms (Parrott, 1995, p.145).

The public "remembers mythical and idyllic representations of the nurse viewed as moral figures above all suspicion in war or healing the residual ills of years of fighting" (Helg, 1995, p.63). They perceive the touching the forehead of the patient as providing comfort and touch and do not necessarily appreciate the assessment skills being used in the gesture. The comfort given by the nurse in washing and changing the clothing of the diaphoretic patient is remembered as comfort, but not recognised as assessment action as the nurse looks for rashes, haemorrhage, pressure, cyanosis or searches for subtle signs signalling the need for urgent attention. The removal of bedpans is a common image of nursing. What is not understood is the assessment and monitoring that this action represents. Over time, some nursing actions alter to reflect changed knowledge and technology, such as early mobilisation rather than prolonged bed rest, showering rather than bed bathing, use of 
special wound care products rather than four-hourly soak dressings, changing position rather than the rubbing action of previous pressure area care. These changes in traditional practice are often confusing for patients or their families who may remember what was accepted practice and do not understand evidence-based practice guidelines. Some actions remain consistent while others change and it this change that requires explanation and reassurance.

In using the 'Contribution Model', it is essential that contemporary practitioners participate in naming the actions taken. It requires a comprehensive knowledge of the context and practice of the profession to ensure that the naming, grouping, analysis, testing and re-framing is accurate and has appropriate emphasis and link to output or outcome. The purpose of naming the actions is to achieve clarity, consistency, simplicity, accuracy and usefulness. Through the process of naming the main activities, it can be anticipated that there will be some debate about the appropriateness or validity of the actions named by the individual or group. This is helpful to the profession as it ensures that there is consistency and linkage.

Naming actions should not to be confused with tasks. Tasks are the isolated events undertaken by someone, although some may be grouped to form actions. Action is a broader concept than task. Action may be a grouping of tasks, for example the task of taking a blood pressure is only part of the action of assessment and monitoring of vital signs. 'Actions' include the descriptions of the synthesis of knowledge, skills and techniques necessary to 
achieve an outcome. To claim an action as unique to that of an individual or group requires that the action is consistently applied by the group of practitioners and is restricted for use only by that group. This may be difficult since much of what is done in health care could be generic tasks. What is unique is how the profession uses the tasks to form actions that will meet the needs of the consumer to achieve outcomes. Nursing is not alone in the need to make explicit the link between expectations, visible action and anticipated outcome. Social work has identified that

the catch [for social work, could read nursing too - my words] is that if the technical base and strength of an occupation consists of a vocabulary that sounds familiar to everyone, it will have difficulty in claiming a monopoly of skill of even a roughly exclusive jurisdiction over its working environment. To the onlookers the practice of social work does not appear markedly different from those of ordinary social intercourse (Howe, 1995, p.142).

The challenge is to understand the anticipated outcomes and show how the actions assist in meeting the expectations and needs identified.

In the case studies used in this thesis, actions are listed to show how the actions link to expectations and how outcomes might be demonstrated. It would be difficult to describe, for example, all actions of the work of early nurses in New Zealand working in response to infectious disease outbreaks. Rather the expectation of the nurse could be described, using modern vocabulary if context-specific description is not available. While historians may have difficulty with use of modern language when relating to past 
accounts, this approach is used in this thesis because past situations are used to show how the 'Contribution Model' works rather than as a formal historical account of society and health in the past. The expectations could be described as disease containment and patient treatment to restore health. The main actions could be described as establishment of an isolated treatment facility (tent hospital); triage and assessment of presenting individual and community needs; care planning for the diverse individual needs; provision of high acuity care practices and treatments for those admitted for care; management of the environment to minimise the risk of cross infection and injury; monitoring of patient progress and documenting outcomes in report; convalescence and rehabilitation strategies including palliative care and management of death; as well as advice to local community leaders on prevention strategies to prevent spread of disease.

These descriptions create a general image of the actions undertaken by the nurse in this context and time period. The description does not fully emphasise the responsibility expected of the practitioner and the resourcefulness of the nurse working in an isolated environment with no back-up services. The initiative and skill required of the nurse is hinted at, namely to deal with all eventualities with minimal preparation and supervision. Narrative would provide greater clarity and texture to the description, hence the use of case studies. The expectations and purpose of the work of the nurse in that setting is stated simply and clearly. The actions are shown to be consistent with the expectations, if one reviews the expectations documented in the Department of Health literature of the time. 
The outputs and outcomes would be visible to all customers experiencing the event or reading about the actions taken because nurses wrote in the nursing journals about their work and also gathered detailed statistics of outcomes for the Department of Health. The contribution of the practitioner and profession becomes clearer despite the absence of considerable narrative detail about the context, or more description of the characteristics of the client group.

'Actions' also alter in response to changed expectations. For example if the expectation is that nursing will provide support services to soldiers in a war zone, or nurses will establish refugee services, then the actions of the nurse will alter accordingly. There will be altered expectation of what actions can be undertaken in that context as compared with a district general hospital in a New Zealand city setting. Likewise changed technology for the surgical management of people with 'fractured neck of femur' will mean that nursing action will alter from traditional management of people in traction to that of early post-operative rehabilitation. The actions component can be individualised or follow a taxonomy or classification system.

\section{Description of actions using classification systems}

For some decades nurses have used medical disease-specific classifications although these did not reflect nursing practice and further increased the invisibility of the profession. More recently the substantive structure of nursing knowledge has been developed to include diagnostic, intervention and outcome concepts. The research of Tripp-Reimer, Woodworth, McCloskey and Bulechek, (1996) and middle-range theory development of 
diagnostic concepts developed by Eakes, Burke and Hainsworth (1998) have developed more detailed understanding of classification systems that articulate interventions and outcomes. Progress has been slowed due to the diversity of contemporary nursing which makes development of classification more complex (Gordon, 1998, p.3).

Classification systems for nursing were given momentum with the work of the North American Nursing Diagnosis Association (NANDA) and were extended further to develop the 'Nursing Minimum Data Set'. The 'Nursing Intervention Classification' (NIC) project was started in 1987 by McCloskey and Bulechek (1996) and is paralleled by initiatives in other countries as there is a perception that the Americanisms behind the words may not allow for a description and comparison of nursing practice across nations. There is a perceived difficulty in testing the commonalities and differences of concepts, values and practices (Clark, 1998, p.2). The International Council of Nurses also began work in 1989, titled the 'International Classification for Nursing Practice' project, and this has proven to be a difficult and slow project. By 1995 every major European country is reported to have developed its own classifications and terminology to meet the imperatives of their local 'market model' and the rapid acceleration of information technology. Attempts to find one approach was slow and is being overtaken by management models that need to measure and forecast. Despite decades of work, there is still no single model for classifying nursing activities and interventions and it is unlikely to occur given individualised developments and rejection of the American models that are viewed as not 'fitting' the 
practice of many countries (Clark, 1998). Descriptions of tasks and lists of nursing interventions need to be balanced with classifications of the type of situations with which nurses deal, the goals towards which their nursing is directed, their modes of intervention and the types or outcomes expected in the client. Having this information captured by information systems allows for easier analysis. A valid and reliable information system also allows determination of competency of the individual practitioner and cumulatively to assess the quality of a nursing service (Gottlieb \& Allen, 1997, p30).

Does it matter that progress has been slow? In my view it does matter because the delay means that the project may not be completed or may be taken over by managers so that it is not nursing-led. There is benefit in having a simple language so that benchmarking is possible between similar practice settings. The need is for a way of communicating in a language that is understood by managers, planners and purchasers who decide on the value of workforce contributions and seek to introduce unskilled workers in a false belief that tasks can be delegated and unskilled is cheaper.

A universal classification model, using the pooling of existing knowledge and best practice, may be the best option given the complexity of development and the growing need for a universal way of communicating what nursing can and is doing. Globalisation of health and information sharing should supersede individualism, regionalism, specialisation and selfdetermination. Care must be taken, however, to ensure that unique models of care and practices do not become 'colonised' by American 'imperialism'. 
Ideally the International Council of Nurses could have acted as the objective integrator of the best work from everywhere rather than needing to start design at the beginning. While there are some gaps in the Iowa Interventions Classification system as regards primary health and the applicability for third world countries, where highly technical practice is restricted and information technology limited, these aspects could be developed with different countries.

Any delay has implications for nursing as the profession may not have the confidence to lead or influence the debate about what it costs for nursing involvement. The delay could mean that nursing interventions are not easily placed on taxonomy lists and future inclusion may be difficult compounding nursing invisibility. In my experience the profession of nursing rarely builds on the success of others to move forward, but fosters diversity, re-does work when it could be used as s step towards further development. While the medical professionals similarly challenge the ideas and practice of colleagues, they do accept evidence and will progress ideas in an intellectual search for new and better approaches. How this will progress is not clear at this stage.

The work of the Iowa Interventions and Outcomes Project (McCloskey and Bulechek, 2000; Johnson and Maas, 1997) provides considerable assistance in standardising the actions of nurses and showing the linkage between identified need, action and outcomes. Examples of this work will be used as headings in later chapters, as the classification is applicable to the New 
Zealand health care setting and could be used to differentiate the work and functions of nurses from that of other health providers in the same practice setting. Given that nurses have difficulty classifying their actions, this system provides a way to communicate in a consistent way as opposed to task lists or bland descriptors such as 'slept well'.

The 'Action' element can be communicated alone but when linked to 'expectations' provides greater clarity. The customer/stakeholder can then evaluate the achievements against their expectations. The 'action' component also interfaces with the 'achievement' component by providing descriptors against which outputs and outcomes can be measured.

\section{Achievement}

The 'achievement' component is the output or outcome phase of the systems model. It is defined as "to reach or attain by effort, acquire, gain, earn, accomplish or carry out (a feat or task), be successful; attain a desired level of performance" (The Little Oxford Dictionary, 1990, p.11). 'Achievement' is used to acknowledge an endpoint or consequence, where what has been attained can now be evaluated. The output or outcomes is made visible, the value of what has been added can be measured using qualitative and quantitative measures. 'Achievement' requires clarification and measurement of what has actually been achieved, using clear and measurable indicators so that what has been achieved can be validated. 
'Achievement' can be evaluated using a number of methods, including audits of 'before and after' states, indicators or measures of productivity, efficiency and effectiveness such as numbers seen, reduction in waiting list numbers, reduced admissions, reduced length of stay. Careful data gathering and analysis is necessary to show impact on health outcome. If anticipated outcomes have been identified in the first phase, the data can be gathered from the beginning. In the 'achievement' component measures, such as timeliness, quality and some subjective values such as satisfaction, can also be included. Qualitative descriptions of effort expended or the level of creativity and innovation demonstrated to achieve an outcome might also be evaluated. These qualitative measures may be as valuable as any quantitative measure and it is recommended to have a mixture of measures to more accurately reflect the range and levels of achievements undertaken by nurses.

'Achievement' is ideally measured by identifying the anticipated outcomes in advance and establishing consistent measurement methods to evaluate the significance of the impact achieved at the endpoint. For example, establishing that the impact of a nurse specialist role will reduce length of stay, reduce the need for admission and enhance the quality of life of the patient or client allows for focused action in gathering information to prove this achievement. With clear identification of main 'actions' or activities, relevant indicators and evaluation criteria, data can be gathered from the beginning and adjustments to practice interventions made so that the anticipated outcomes can be observed. Use of validated and relevant tools as well as timely and appropriate data can then be gathered to measure what has 
been achieved. Using and measuring the right indicators is essential to provide evidence to the stakeholder of what has or can be achieved. Finding the right method for measuring achievement might require some coaching. The 'Nursing Outcomes Classification' (NOC) publications (Johnson and Maas, 1997) provide validated tools for use. Some practitioners might find it easier to start with measurement of achievement of goal weight; stabilisation on treatment plan within agreed time; reduction of crises through effective self management; reduced number of admissions; or shorter length of inpatient stay. Quantifying the achievement in economic terms is of increasing importance. In the current environment of economic rationalism, the greater the validity of the link between the expectation, action and achievement, the greater the appreciation of the value of the contribution.

The 'Achievement' component is interrelated with the 'expectation' and 'action' component of the model. Without clarity in the first two major units of the 'Contribution Model', the measurement of outputs and outcomes is difficult and can be resource intensive. The 'achievement' element provides the measurable evidence of contribution.

\section{Responsiveness}

The 'responsiveness' component is considered the feedback loop in this model. Responsiveness is defined as "responding readily to some influence, movement or change caused by a stimulus or influence" (The Concise Oxford Dictionary, 1990, p.1026). 'Responsiveness' is the integrating element that highlights the ability of the provider to respond to any situation, 
to be creative and innovative, to adapt knowledge and experience to diverse situations in order to achieve a positive outcome. The 'responsiveness' component provides a way of acknowledging the flexibility and adaptability of the provider in response to changed expectations or requirements. 'Responsiveness' is linked with the 'expectation' and 'action' major units and monitors the achievements to ensure that if the outcomes achieved are not consistent with expectations, then the actions are adjusted and the reaction from the stakeholders is monitored as well. Responsiveness is measured through indicators such as timely response, initiative, innovation, change and leadership.

'Responsiveness' is the ' $\mathrm{x}$ ' factor, noticeable when absent. This component monitors the environment and the effectiveness of the link between expectations, actions and outcomes and influences change so that there is evidence of listening, reflection and timely change where necessary. Responsiveness is the component that ensures professional survival and continued relevance.

\section{Crafting the model into a usable framework}

In order to make the model more user-friendly, the main major units of the 'Contribution Model' have been formed into a useable framework. The framework guides the user through the steps to articulate the expectations of the various stakeholders, the actions (to be) taken in response to the expectations, the achievements identified and the indicators of responsiveness. The framework is used extensively in each case study and scenario in the chapters that follow. 
The major units of the 'Contribution Model' are documented using the following format:

Table 1. Framework tool to use in applying the 'Contribution Model' major units.

\begin{tabular}{|c|c|}
\hline $\begin{array}{l}\text { Socio-political } \\
\text { context }\end{array}$ & Specific to the case study or scenario \\
\hline Health issues & Specific to the time period and issues arising \\
\hline Nur & period and phase of development \\
\hline $\begin{array}{l}\text { Expectation } \\
\text { is understood by } \\
\text { clarifying and } \\
\text { agreeing the }\end{array}$ & $\begin{array}{l}\text { Identity, characteristics and expectations of the } \\
\text { different Client/ Recipient/ Stakeholder groups } \\
\text { The nature and context of the service to be provided - } \\
\text { purpose and function of role/work to be done } \\
\text { The characteristics of the provider - knowledge, } \\
\text { skills, and experience required } \\
\text { Goal or outcome - anticipated outcomes }\end{array}$ \\
\hline $\begin{array}{l}\text { Action } \\
\text { is understood by } \\
\text { describing the }\end{array}$ & $\begin{array}{l}\text { Main focus or activities of the role expected, not } \\
\text { individual tasks }\end{array}$ \\
\hline $\begin{array}{l}\text { Achievement } \\
\text { is measured } \\
\text { through evidence } \\
\text { gathered about }\end{array}$ & 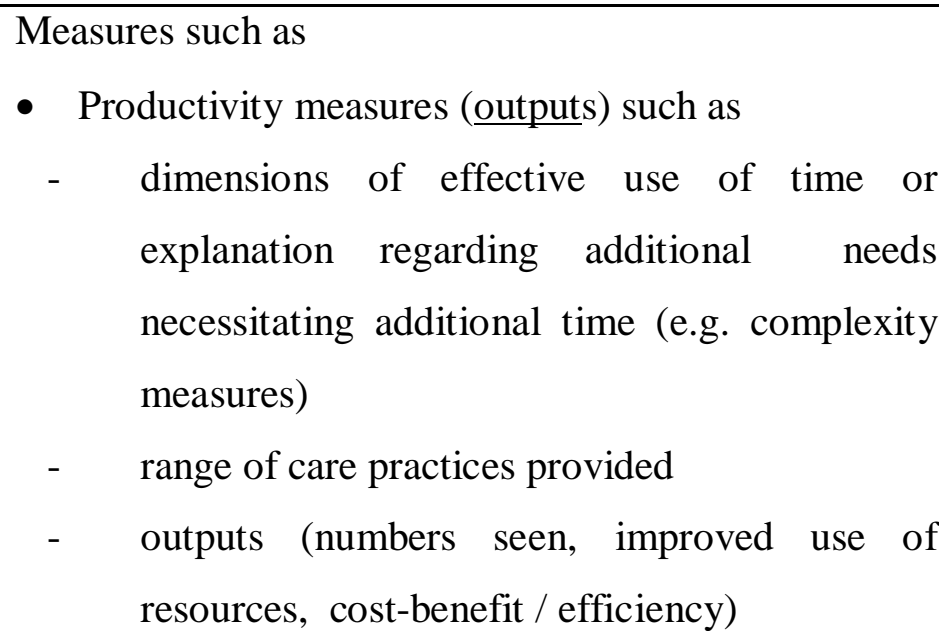 \\
\hline
\end{tabular}




\begin{tabular}{|c|c|}
\hline $\begin{array}{l}\text { Achievement } \\
\text { continued }\end{array}$ & $\begin{array}{l}\text { Outcome measures for } \\
\text { effectiveness e.g. reduced crises and self-initiation } \\
\text { of Asthma management plan to minimise need for } \\
\text { hospital admission } \\
\text { - goal-achievement as agreed between all parties } \\
\text { (lifestyle changes, improved coping with health } \\
\text { problem, functional improvement) } \\
\text { - impact if intervention on quality of life } \\
\text { - satisfaction of the consumer, the value placed on } \\
\text { the partnership and shared relationship between the } \\
\text { consumer and provider; value of effort expended } \\
\text { to achieve expectations e.g. build trust, } \\
\text { perseverance } \\
\text { the value placed on the process of learning on the } \\
\text { way stated satisfaction with the consistency } \\
\text { between achievement, the expectations and/or } \\
\text { anticipated outcomes } \\
\text { the significance of the impact/'added value' on } \\
\text { health gain the difference achieved through } \\
\text { involvement and work done }\end{array}$ \\
\hline $\begin{array}{l}\text { Responsiveness } \\
\text { is identified by } \\
\text { descriptions of } \\
\text { actions taken, } \\
\text { such as }\end{array}$ & $\begin{array}{ll}- & \text { readiness to respond (preparation, timeliness) } \\
\text { - } & \text { evidence of anticipation, proactive action } \\
\text { - } & \text { adaptability } \\
\text { - } & \text { innovation and creativity } \\
- & \text { scope of response } \\
- & \text { quality assurance } \\
- & \text { relevance } \\
- & \text { presentation, reporting }\end{array}$ \\
\hline
\end{tabular}

The user of the framework is then recommended to work through the elements under each component. In effect this framework provides a three dimensional perspective, encouraging description about the context, namely 
what is happening in society to influence the expectations, what is happening in health as a system within the social system and then what is nursing doing to respond to the expectations.

In this chapter the 'Contribution Model' follows the same pattern as the General Systems Science and Cybernetic Theories using the core components of input, process, output and feedback pattern. This pattern provides a simple, clear guide for use, that is self-evident, that uses language and patterns that are known to most nurses through education and practice. The theory is systematic, logical and credible. The framework is userfriendly and multi-functional, applicable to a diverse range of situations and complex structures, systematically gathering ideas so that there is a balanced presentation of information. The language used aims at being universally understandable for clinical staff as well as managers, accountants and health planner. The model allows for use of real examples that can be validated, integrating ideas from contemporary research. The model allows for comprehensive presentation of information that can be presented confidently to articulate practice and the contribution of the practitioner.

The model and the major units identified provide a way of structuring ideas and information and validate the relevance of the concept of 'contribution' to nursing. The model is based on a Systems Science and Cybernetics Theory, which is relevant for nursing because nursing is a subsystem, a socially constructed professional response that works within and amidst a range of systems and organisations. Nursing is perceived as "a consciously co- 
ordinated social entity with a relatively identifiable boundary that functions to achieve a common goal or set of goals" (Robbins, 1990, p.4). Nursing, as a system within systems, works to produce an output or outcome to meet agreed expectations or may co-ordinate inputs of interrelated, interdependent parts/individuals and groups and to achieve a unified whole/outcome. The model provides a structured guide for identification of expectations, clarification of actions, identification of measures for evaluation of achievements and acknowledgement of responsiveness. Understanding the model is essential because in future chapters the 'Contribution Model' is applied using case studies using information gathered from historical events, observation of contemporary practice and use of scenarios to consider nursing's future contribution to the health of New Zealand.

\section{Evaluating the 'contribution model'}

There are a number of criteria used to evaluate the validity of a model. This section outlines a range of accepted criteria that could be used and evaluates whether the 'contribution model' meets these criteria and can therefore be considered valid.

The most often discussed criteria are clarity, simplicity, generality, empirical precision and derivable consequences (Tomey, 1998). Other criteria are also used to evaluate effectiveness, including that the model must be unified and present a sequenced design of concepts and principles that can be used to describe the arrangements of and relations between the parts to connect them into a whole (Heylighen \& Joslyn, 1992). A conceptual framework or model 
must also be based on well-established theory, to demonstrate correspondence. The model must be clear about what the model is supposed to do, that it is coherent. It must be simple and applicable to minimise fault and be based on clear requirements of application, to be consistent. These criteria will be some of the criteria used to evaluate the value of the 'Contribution Model'.

There are also other properties of evaluation that establish the stage of development of a model, evaluating whether the model is primitive, at the beginning to a system of thought or something that can follow from the initial thoughts and is therefore more sophisticated. Another criteria is that of the simplicity of the model, so as to be self-evident or tautological. A model is also evaluated according to the criteria of universality as regards having universal applicability. In developing a model the aim is to arrive at a network of concepts and principles similar to a formal system, with definitions of higher order concepts and theorems derived from the more primitive axioms and definitions (Heylighen, 1992, p.4).

In constructing a conceptual framework or model in a complex dynamic system, a number of other principles are also acknowledged: complexity which relates to complex structures with many heterogeneous interacting components; mutuality where components are interacting in parallel, cooperatively and in real time creating multiple simultaneous interactions among subsystems; complementarity where subsystems participate in multiple processes and structures; evolvability whereby systems evolve and 
grow in an opportunistic manner rather than designed or planned in an optimal manner; constructivity which refers to how the system increases in size and complexity simultaneously binding to previous states while developing new traits; and reflexivity which demonstrates that through both positive and negative feedback the system is able to initiate self-reference, self-modelling and self-production.

\section{Application of criteria to the 'Contribution Model'}

Using the criteria above, the 'Contribution Model' proposed in this thesis is discussed. The 'Contribution Model' is based on the well-established 'General Systems Theory' and this can be seen in the correspondence between the formal theory and the model components, of inputs, process, outputs and feedback loop.

A system is defined as the relationship between things that interact, a functional whole composed of a set of subsystems and components that when coupled together generate a level of organisation that is fundamentally different from the level of each individual subsystem. The 'Contribution Model', places nursing as a subsystem within another subsystem of health care delivery which is also a subsystem of society as the wider system. Nursing acts to meet the priorities of the wider health system and society, responding to the expectations of each, even when they may have different and conflicting agendas. The component parts of the General System Theory relate to those of the model in that the inputs are articulated as expectations, the process is described as actions and the outputs are communicated as 
achievements. The feedback loop is identified as responsiveness. All of the component parts interact in a unified manner, linking expectations to actions, actions to achievements and expectations to achievements. The concepts are sequenced as a design of concepts and principles that can be used to describe the arrangements of and relations between the parts to connect them into a whole. The model has a network of concepts and principles similar to the formal General Systems Theory and there is an attempt to define the concepts, to provide definitions of higher order concepts.

The General Systems Theory model, upon which the 'Contribution Model' has been based, was used because nursing, as a social agent and a profession, is part of a number of intersecting systems. Nursing as a profession exists as a subsystem of the world of health service provision. The 'world' or wider system has expectations and 'laws' about how the subsystems will interrelate to achieve the shared goal of service provision and achievement of health goals. There are accepted processes and anticipated actions that will occur by the subsystem to achieve the desired outputs. The outputs are evident and observable even if all of the actions of the agent are not. Contribution, as a concept, has been shown to comprise inputs and expectations that are linked to the actions and processes undertaken to achieve observed and validated outcomes and outputs. The actions of the subsystem are responsive to change in the wider system, influenced by the feedback loop. The expectations, actions and achievements of nursing as a subsystem are influenced to be responsive, allowing for change, movement and growth. The 'Contribution Model' has a number of elements under the main input, process and output 
components. The elements under Expectations requires research and analysis of the expectations of the stakeholders, clarification of the purpose of the work expected, explanation of the knowledge and skills required to meet the expectations and confirmation of the anticipated outcomes. Action requires description of the main groupings of activity that will be undertaken to achieve the stated expectations. Achievements are described using measures of outputs and outcomes. Responsiveness is the feedback loop that represents the way in which the sub-system adapts in response to environmental scanning and changes in expectations and actions.

The 'Contribution Model' has been described coherently in various contexts, using the associated framework tool to show its working parts when comparing case study and scenarios. The flow of the model is used over and over in the case studies using practice in historical contexts, contemporary practice roles and to articulate the contribution of nursing in a range of possible futures. The repetitive use of the model demonstrates how the model works to provide a description of the responsiveness of nursing to the changing expectations of the profession. The repetitive use indicates that the model is consistent and robust when applied to a variety of situations.

The model is potentially simple and relatively easily applied. The aim was to provide a user-friendly model that could be used in a range of situations with minimal explanation. The intention was that the model could work as a tool to enhance articulation of the contribution of nursing using simple components and could be used by anyone because of the link to a well- 
known theory. The development of a framework tool to guide the use of the model components was also aimed making the conceptual model easier to use. While confident consistent, systematic application of the model will require some practice, the framework fosters a comprehensive overview and discourages over-development of one aspect. The language used in completing the framework can be simple, contemporary and unambiguous rather than complex and overly precise.

The 'Contribution Model' could not be considered a primitive model, in that it has moved past the beginning ideas to a system of thought that links with other researched complementary developments, namely the nursing Intervention and Outcomes Classification. The model can and will continue to develop as people use the concepts and apply the elements in a different way. This does not imply lack of development but reflects the need for application by practitioners in practice settings.

The model has universal applicability, in that it can and has been shown to apply to the full scope of nursing roles and functions in a range of contexts. The model can be used as a coaching tool, where a person needs to understand their new role or to refocus on their existing role. It can be used in the development of job descriptions; and in the development of service plans, where there is little known about the expectations of the various stakeholders, the purpose of the work to be done and the knowledge and skills required in order to decide who might best meet the identified needs. The model can be used to negotiate finite resources where there are multiple 
and competing expectations. The model can be used where a project needs to be evaluated. The framework may be more useful before the project starts rather than after a period, as data needs to be collected from the beginning rather than collected in retrospect.

The model can be applied where there is complexity, with complex structures with many heterogeneous interacting components. This was demonstrated where nursing as a sub-system was described amidst complex social and health demands. Health provision is a complex process of interaction with multiple stakeholders, each with their own priorities through which nursing must work co-operatively so that the competing priorities of needs and goals of the individual, group and community are achieved. The model has been shown to have mutuality, in that components can interact in parallel, cooperatively and in real time creating multiple simultaneous interactions among subsystems. Responsiveness is the feedback loop that ensures that there is interaction and adjustment where there is a change in expectation so that actions match expectations, and where achievements are not met then actions can be adjusted to ensure that anticipated outcomes do have the desired effect.

The model demonstrates complementarity, in that subsystems that participate in multiple processes and structures remain functional throughout. The model requires articulation of the inputs, actions and achievements and these components are in constant interaction with other subsystems, processes and structures. Inputs are derived from multiple sources that change with 
changing social experience, new technology or reaction to crisis. Actions alter in response to new evidence, new learning and new technology. Achievements alter in response to changing knowledge and expectations of rigour in measurement.

The model meets the criteria of evolvability, in that the system elements can be different and evolve rather than requiring rigid compliance with the designed and planned elements. The model allows for adjustment as new knowledge is published, as technology allows for greater capture of data and as new roles arise.

The model meets the criteria of constructivity, in that as the system increases in size and complexity, there is simultaneous binding to previous states, while developing new traits develop. This is seen in the retention of core values, philosophies and some practices despite changes in technology and learning. The model can have more elements added as the model is used. There will be elements that will alter over time in order to establish clarity. As seemed appropriate, the Nursing Interventions Classification (NIC) taxonomy was used to provide structure to the descriptions of actions undertaken by contemporary nurses. This integration allows the model to grow in size and complexity depending on the issue under discussion and the audience to whom the information is directed.

The model also meets the criteria of reflexivity, meaning that through both positive and negative feedback, the system is able to initiate self-reference, 
self-modelling and self-production. The feedback loop, titled responsiveness, ensures that the subsystem is open to receive information that can alter the expectations and this information is passed to the actions and achievements so that adjustments can be made accordingly. The framework that uses the component parts guides the user to complete the details against the backdrop of social and health-related events. The events alter but the framework acts to reflect the contribution of nursing against whatever background evolves.

Semantic and structural clarity and consistency are evident in the limited use of major concepts and sub concepts. There is definition of the concepts expectations, actions, achievements and responsiveness and an attempt to use the general meanings that apply within and across disciplines. Diagrams and examples have been used carefully and consistently. The logical development is clear and the assumptions consistent (Tomey, 1998, p.8).

While the model meets the simplicity criteria by using a few concepts only in a simple relationship, it is also sufficiently complex to provide a sense of understanding through concrete descriptions (Argyris and Schon, 1974, p.198). The conceptual model also meets the generality criteria, in is broad scope and the potential application to many situations (Chin \& Jacobs, 1979, p. 239).

The model meets many of the empirical precision criteria, in the 'testability' and use of the model, with the concepts grounded in observable reality. The model is presented as a theoretical work and requires other researchers to 
evaluate and verify the results, even if it relates to scope, complexity and clinical usefulness. The criteria of derivable consequences relate to the usefulness of the model in relation to how it develops and guides practice. The theoretical application of the model demonstrates how this might occur in practice and should be tested.

The 'Contribution Model' meets the criteria identified as relevant for evaluation. The 'Contribution Model' has been used throughout the thesis and has demonstrated functionality and applicability. The 'Contribution Model' provides new knowledge, a new way of considering and communicating contribution. A similar model was not found in literature reviewed. The model complements the developments of the Iowa project and therefore has additional functionality as it is flexible and is able to integrate with new ideas.

The 'Contribution Model' as made a contribution to the discipline of nursing and to other users because of its simplicity in concept, its systematic and logical method. It can be used with minimal explanation and becomes a systematic way of thinking and communicating. The 'Contribution Model' is based on a theory that is generally known, understood and used by nurses and other groups. The main concepts are known so that the new interpretation can be built on this foundation for ease of use. The 'Contribution Model' is user-friendly, is simple to grasp; it is relatively uncomplicated in design and can be used in multiple ways. It can be used for 
situations where it may be useful to understand multiple expectations and negotiate expected outcomes to meet those expectations.

The 'Contribution Model' uses a structured framework to guide the user to systematically gather ideas in a balanced manner that does not over-simplify or over-complicate. The framework guides the user to document using language that is understood by decision-makers, planners and practitioners and provides a common base for communication. The details in the framework present a confident professional profile. The 'Contribution Model' allows the user to use real examples rather than stifle their presentation using language they are not comfortable using. It integrates with developments and research, is flexible and adaptable.

The 'Contribution Model' structures the ideas about nursing contribution against the context and expectations of society and health service provision and show the nursing response to the identified need. The model provides focus in the succinct description of what might be done and for what reason. For a health planner, the model and framework provides an overview of what might be possible and what knowledge and skills lie beneath the actions that are visible. It allows the planner to identify the scope of practice and areas of gap that might be complemented by other health providers to complete the matrix of expertise. The framework allows for negotiation of scope of practice and agreement of the anticipated outcomes to be achieved. The framework provides more value when applied to a specialised context or expectation as the match between expectations, actions and achievements can 
be more specific. As an overarching description, the 'Contribution Model' encourages more succinct and detailed description of the function and actions of nursing than would evolve using a more open-ended narrative approach.

In this section the 'Contribution Model' and framework has been evaluated using key criteria acknowledged as relevant for reviewing the validity of a model. The 'Contribution Model' has demonstrated appropriate 'fit' with the requisite criteria.

In the next chapters, the context of practice, namely health, is explored as a dimension against which nursing practice will be profiled. The model will be applied using three time periods, historical, contemporary and future, to profile nursing and its past, present and future contribution. 


\section{Chapter Four}

\section{Health: the constant priority of a nation}

Health and its importance to survival and social progress cannot be overstated. This chapter focuses on health, health care priorities and the expectations that society has of those approved to maintain and restore health. This chapter 'sets the scene' because health and health care is the context within which nursing works. The priorities and changing expectations in regard to health and care interventions are the inputs to which nursing processes respond. Health encompasses disease and non-disease and it is across this continuum that nursing works to maintain or restore the client's independence. Health is the ultimate achievement and is the goal towards which nursing action is, and should be, directed. This chapter explores why health is important, how heath has different meanings for different groups and that that public health priorities change in emphasis over time. This is relevant for nursing and the discussion that follows because the work of nursing is and has been about working with people to achieve a shared meaning and goals and to respond to changes in expectations over time.

\section{Why is health important?}

Good health is acknowledged as one of the major factors impacting positively on social and economic structures. Good health enables people to participate more fully in society and contribute to community productivity by pursuing their goals in life (Howden-Chapman \& Cram, 1998). The health of 
community members has been viewed as essential to the success and survival of societies and it has been the aim of society and social agencies over time to maintain and restore people to the best health possible in order that they may contribute to social development. The roles that society wants and expects from nurses and other health workers, is to provide care for the injured and unwell as well as to educate them to maintain their health. These roles have, in most instances, been viewed as a valuable asset since they have promoted health maintenance, disease containment and have assisted with recovery and rehabilitation.

Health gain is the primary goal of the various health service provider groups. Nursing works primarily in the domain of health, in the promotion and maintenance of health, in the initial assessment and treatment of acute illness, in the focus on health recovery and rehabilitation and supporting the quality of life of those people who are dying. The expectation by society that health recovery was the primary aim of health services has influenced the work undertaken by nurses and the demand for nursing input in the acute illnessrecovery process. While disease prevention and health maintenance has been important part of nursing work, the saving of life and restoring of health has consumed more and more nursing resource as technology has made this possible. In the end health is important because health provides a heightened awareness of the meaningfulness of life. 


\section{Health: different meanings and expectations}

From the beginning of social organisation, the preservation and protection of the health of each community has been a consistent motivation. This section considers three linked ideas: that maintaining health has been a priority for communities from the beginning of time; that communities have always needed some members to take responsibility for the care of those not in full health; and that over time there has been an alteration of what has been expected of care givers. It is not intended in this section to provide a historical description of the developments from 'hunter-gatherer' to modern day healthcare as that is a thesis of its own. Rather the context of health will be explored as the focus of nursing interventions.

Consistently through history, individuals have needed to be in good health in order to forage for food, to care for their families, to protect the group and to undertake social responsibilities that promoted the well being of the group as a whole. Individuals who were unwell added a burden to the group. There was reliance on some group members who were experienced in the use of resources to restore the health of others as promptly as possible and to prevent the spread of disease. Many communities organised the care of the ill to minimise the burden of care.

The organisation of health services has evolved over time with increasing role differentiation of caregivers able to assist in health restoration and care provision. In identifying roles, societies acknowledged the value of those individuals identified as skilled in treatments and health care (Donahue, 
1985). Nursing, as one of the disciplines that emerged in health care provision, is a practice discipline that has developed in focus and function over time, responsive to social developments. Nursing the sick was not, however, identified as a professional activity by people in the ancient world (Hudson, 1998, p.7); the professionalism of nursing is a relatively modern phenomenon.

What individuals and communities meant by the concept of health and healthfulness has altered over time. Disease has been viewed by some as an independent force that dominated the individual. Some have viewed wellness as the absence of disease, with many perspectives influencing priorities in service provision and in research programmes. The medical-physiological perspective considered health as the resistance of the individual to disease. The ecological perspective viewed the environment as influencing health. The equilibrium perspective considered ill health as ineffective attempts to maintain homeostasis or adaptation. The social view considered health as the ability of the person to effectively perform social functions. In contemporary discussion health is considered a state of complete physical, mental and social well being, an ever-changing state of being from high level wellness to extreme poor health with death imminent. Health can be described as:

a purposeful, adaptive response, physically, mentally, emotionally and socially, to internal and external stimuli in order to maintain stability and comfort... The key concepts in health and wellness include homeostasis, adaptation, the dynamic nature of health-illness continuum, the influence of internal and external environment, 
comfort, safety, social relationships and the prevention of disease, disability and social decay (Murray \& Zentner, 1979, p. 5-6).

Regardless of the perspective taken, the emphasis is that health is a balance in adapting to the stimuli that can result in ill health.

Since the mid-1800s health services, in New Zealand and internationally, have been increasingly organised to maintain health and treat illness, expanding as technology has allowed more active and effective intervention. The emphasis on health promotion, disease prevention, treatment of illness, health restoration and support of those dying has remained constant. Human concerns, such as poverty, living conditions and welfare support of those unable to care for themselves, have similarly remained constant (Donahue, 1985). With scientific development initiatives to prevent illness through immunisation, the promotion of healthy living has increased in emphasis in some countries. Use of new diagnostic technology, earlier diagnosis, noninvasive interventions and new treatment methods have expanded health service options requiring more of health providers than ever before.

To make sense of the range of services offered, health planners and service providers have emphasised a systems approach to achieving health gain. Health, however, is a relative concept that each individual and community defines for themselves, which may not easily fit with a systems 'cause and effect' paradigm. Health for some may have little to do with systems and interventions, but be more about personal experience, culture and social circumstances. Theories of health focus on health as an ideal state, with the 
goal of perfect well being. Some groups, however, consider health as the physical and mental fitness to do socialised daily tasks; whereas others may view health as a commodity that can be bought or given; or health is a personal strength or ability either physical, metaphysical or intellectual (Seedhouse, 1997, p.29). Seedhouse suggests that instead of one perspective, one should understand the differing perspectives and consider three approaches designed to increase health. He suggests the sociological approach that focuses on the factors that influence health; the approach of medical science that focuses in the causes of disease and treatment options; and the humanist approach that recognises self-responsibility for health as the ultimate goal. The sociological understanding of health is the broadest perspective that acknowledges that life experience is influenced by cultural and social background, geographical location, expectations derived from current knowledge and available resources all impact on health. This broad approach allows health workers to work holistically in seeking the approach that will engage individuals and communities in self-responsibility.

Health as a contemporary aspiration considers well being and health as more than the absence of personal illness or injury. Recent definitions include: a state of complete physical, mental and social well-being, the richness of individual's social networks, a sense of community and the social capital of each community, the accumulation of good-will and trust built up from individual participation in organisations outside the family (World Health Organisation, 1986; Howden-Chapman \& Cram, 1998). 
The Ottawa Charter for Health Promotion has described "health as created and lived by people in settings of their everyday life; where they learn, work, play and love" (World Health Organisation, 1986). This was reinforced by descriptions from the Mental Health literature, which describes the concept of health as

clearly influenced by a person's cultural values and beliefs and includes concepts of a person's psychological, social and behavioural functioning in their world and their sense of ease with this. This is evident in their psychological clarity and efficiency, their moods and feelings, their ability to cope with the demands of life, their satisfaction with relationships with others, the extent to which their ethnic, cultural and spiritual needs are being met, their sense of value and place in the community and world (Ministry of Health, 1997).

Health can be viewed as a shared responsibility, at an individual-family unit level and a community-society level. Society, while contributing to the goal of health through targeted interventions, cannot impact on individual spiritual and cultural health except to create support structures and influence social opinion to recognise the unique needs of diverse cultures. The concept of health is broad and all encompassing (Latimer et al, 1969). It is within this breadth that nurses, as individual members of society and as agents of society, act to promote health practices, maintain health to the highest level possible and to contain disease where it has the potential to impact on the health of the community. 
The elements identified as important to maintain health vary between cultures. For Maori and Pacific Island peoples, the concepts of health tend to be more holistic, broader than the physical focus on absence of disease or infirmity. For Maori, concepts of health are focused on cultural concepts and practices (Durie, 1996, Pere, 1988). A number of models of Maori health have emphasised different dimensions of health not emphasised by the European definitions. Whare Tapa Wha (Pomare, 1995) is described as the four cornerstones of Maori health that contribute to well being: te taha wairua (spiritual aspects); te taha hinegaro (mental and emotional aspects)'; te taha whanau (family and community aspects); and te taha tinana (physical aspects). The Te Wheke model (Pere, 1984), describes the dimensions of health that lead to waiora or total well-being as: wairuatanga (spirituality); hinegaro (mental); taha tinana (physical); whanaungatanga (the extended family); whatumanawa (emotional); mauri (life principle in people and objects); mana ake (unique identity); and ha a koro ma a kui ma (inherited strengths). These models are important for health providers to understand because these are key concepts relevant to the practice of health by the indigenous people in contemporary New Zealand. These models may not be explicitly articulated by all Maori but are intrinsic to the way in which they respond as a community to health promotion education and the importance of spirituality and family when an individual is unwell. Failure to understand and acknowledge these perspectives means that health workers do not have influence with these groups. 
The Ministry of Health publication He Kakano Oranga (1998a) acknowledges that while social, cultural and economic factors are the main determinants of health, all members of the community have the following right: to good education, good health, good food and full employment; a home free from violence; self esteem and confidence, knowledge and understanding of Maori identity, te Reo (indigenous language), Maori culture and tikanga, full whanau, hapu, iwi or modern configuration support to ensure continuity of Maori knowledge, information transfer and whakapapa (identity); a healthy environment, good sanitation, water quality, economic security and adequate recreation. These descriptions focus on health as more than applying to an individual, rather identifying the responsibility for health as that of society as a whole. Ensuring that all of these dimensions are addressed to achieve health gain places a challenge on health planners and politicians as well as health providers and community leaders.

While Maori, as tangata whenua, have articulated the broad dimensions considered important for health maintenance, these concepts are also consistent with those of many other indigenous cultures. There is evidence that where these components of health are downplayed in any culture, there are poorer outcomes, restricted use of existing health services and increased communities at risk (Smith, 1991).

Increases in the quantity and quality of health services will not necessarily result in improved health status. In fact deteriorating health indicators may occur because of social poverty, environmental contamination and isolation 
from social supports. For nursing to undertake health work with diverse communities, it is essential that there is understanding of the perceptions and expectations of the various cultural groups and all stakeholders and that nursing is given legitimate authority to co-ordinate services to address the identified needs. Without this the nurse will not be successful in assessing need or working in partnership with the individual or group to achieve health goals. Health gain requires a partnership, dependent in part on the involvement of the individual or group to identify what a healthy state might mean for them and also to work with a broad platform of initiatives, including health promotion, disease prevention and illness recovery.

New Zealand has evolved a particular perspective of health, influenced by social and cultural perspectives. Considerable work has been undertaken to develop shared ownership of problems resulting from unequal access to services. There has been acceptance that there is no single solution and that promoting and maintaining health can be done in many ways as long as the goal is achieved (Public Health Commission, 1995, p.9). This perspective has evolved over decades and acknowledges that

a healthy society has structures and practices that treat all members humanely and equitably and works to ensure economic and ecological stability. Integral to this is the acknowledgement that everyone should have access to healthy living conditions and economic circumstances that support their wellbeing. A healthy society acknowledges and responds to the special needs of people with mental disorder, illness, disability or vulnerability. A healthy society affirms that each 
individual has value and that communities have the capacity to make informed decisions, to participate in determining solutions to community problems and to nurture and care for their members (Disley, 1999, p.4).

This perspective of health brings together the various strands of expectation so that health providers can consider health in its broadest perspective and as a shared responsibility consider the range of approaches that will have an impact.

Social responsibility for the health of the community has underpinned the aspirations of the New Zealand welfare state since the late 1930s. From early colonisation to the 1930s, inpatient health services were provided for the Maori and those without social supports but it was expected that the general public were self-reliant. In the early settlement period, health planners were despairing of the "sentimentality" and reliance of the community on social support (AJHR, H-31, 1897, p.1). The provision of free health services arose following the Social Security Act 1938 in response to the Labour government ideology and response to the impact of severe poverty evident during the economic Depression. The Labour politicians considered that society needed to be responsible for providing for the needs of its members in order to achieve a strong community that could participate in future social and economic development. This change in policy, from self-responsibility to the state as provider of social services, had a profound impact on the ongoing expectations of New Zealand as regards free health services. Free health services increased the demand for hospital and home-based care. While 
there was a complementary emphasis on health promotion and disease prevention, the enthusiasm for free hospital care was more marked. What the broader perspective about health requires is action on all 'fronts', dealing with social support networks, access to healthy living conditions, food, education and employment. There is increasing evidence that this broad perspective and action achieves a health community but

It is difficult to quantify the positive contribution and direct relevance on health of good housing and transport, high incomes, well educated people, good social welfare services and good public and environmental health service (Hyslop, Dowland \& Hickling, 1983, p.211).

The view that good health is more likely to be attained and maintained where there is relative stability and a high level of economic well-being was acknowledged by Florence Nightingale (Nightingale, 1859). The relationship between health status and economic security had been considered to be a factor in health gain and this influences the decisions of health policy, planners and strategists. Governments have been lobbied to address the causes of poor economic performance and inequity in the distribution of resources in order to improve and maintain health status.

In New Zealand, health statistics reported in various publications over the past century would suggest that health initiatives have been more successful in controlling disease and provision of treatment services (Ministry of Health, 1998b; Hyslop, Dowland \& Hickling, 1983; Dow, 1995). Emphasis 
in recent years has been on a comprehensive integrated approach to service provision and there is still more work to be done to address all aspects of the continuum from health promotion to symptom management. In this section, the broad perspectives on health have been presented to show the influences on thinking of health planners, service providers and communities. These influences are at times narrow, divergent or holistic and inclusive. As priorities for survival have lessened in aspects such as infectious diseases, the ability to consider wider imperatives for health expand the expectations and places where nursing might work in the future.

\section{Health: the nursing perspective}

The work of nursing in relation to health and health recovery has been more than as an agent of society "to prevent disease, prolong life and promote health". Early descriptions of the purpose of nursing placed the profession with "the sick, suffering and helpless" ( ${ }^{1}$ Kai Tiaki: The Journal of the Nurses of New Zealand, 1908), "and the giving of ease and (physical) comfort to the patient" (Kai Tiaki, 1911, January, p.76); less in the domain of health promotion. These are the first documented descriptions of the purpose of nursing in New Zealand. They provide a useful insight into the scope of practice that nursing defined for itself in those early days and that influences the vision of nursing by nurses today.

Florence Nightingale, however, had recognised the influence of social, political and economic structures on health status and the need for nurses to

\footnotetext{
${ }^{1}$ Kai Tiaki will be used as shorthand for Kai Tiaki: The Journal of the Nurses of New Zealand and subsequent titles changes.
} 
take action on a broader front. She encouraged nurses to address the environment as the origin of conditions that lead to illness (Chopoorian, 1986, p.39). Nightingale recommended that nurses consider "the five essential points in securing health: pure air, pure water, efficient drainage, cleanliness and light" as these aspects, in her view, influenced health outcomes and did not constrain nursing to the 'sickness-care' role (Nightingale, 1859). Nightingale did have evidence for her perspective on health as being more than treating the illness manifestation. She became a strong advocate for identifying the cause of ill health and gathering epidemiological information to demonstrate health improvement (Keith, 1988). This broad perspective on health was a significant contrast to the illness-treatment role that narrowed the role and function of nursing. Nightingale envisioned nursing as having a wide sphere of influence in health and service provision and she recommended that practitioners receive a comprehensive preparation for practice in order to work in the domain of health.

New Zealand nurses in the late 1800 s were prepared educationally and experientially to work anywhere, from the management of the environment and provision of health care to roles that minimised risks and restored health. While nurses worked either in in-patient facilities or in home-based nursing, many were adventurous and willing also to undertake the early role of 'backblocks' nursing in isolated, under-resourced settings. These nurses influenced improvements in environmental hygiene, in childcare, in nutrition, in fever and infectious diseases nursing and in the homes of the isolated 
settlements. The work of nursing in the field of health was broad-based, not just focused on treatment of illness, but including the support of isolated families to maintain health and increase self-sufficiency. As the threat of infectious diseases receded, because of new medicines and co-ordinated environmental management, nursing become more specialised, focused on treatment and health recovery.

Fewer nurses and allied health groups are now involved in health promotion activities, with the work of public health becoming a speciality in its own right. Health promotion is not an integrated part of the practice of every community nurses, as it had been in previous years. Disease prevention, health promotion and health maintenance is now the focus of a smaller number of nurses in defined roles. Correspondingly, the range of roles for nurses in the treatment of disease and restoration of health after ill health has increased with new specialisation and less emphasis on health promotion. Promoting health and self-responsibility is an area where nursing may like to refocus in the future.

Nursing has not necessarily claimed 'health care across the continuum' as its prime focus as a discipline, nor has the profession sought any specific niche as its special domain. Rather, nursing has continued to work in all spheres, across a broad spectrum of care settings. In so doing the profession as a whole has not achieved a high profile for actively promoting health or being consistent activists to improve the social determinants of health. Nursing, as a collaborator in strategies to improve health, has not demonstrated 'fearless 
leadership' in any one sphere; nor has it advocated "for restructuring of societies or for social transformations, even though nurses are eye witnesses to the most deleterious effects of the environment... the effects of illness in individuals who lack housing, jobs, healthcare, schooling and food" (Chopoorian, 1986, p. 40).

This limited focus on health promotion or disease prevention and corresponding emphasis on treatment interventions could be a reflection of the influence on nursing of medical specialisation. The focus on nursing therapeutics has educated nurses about the activities and actions used to care for patients or clients. The nursing process has taught ways of assessing, diagnosing and intervening. Health maintenance as the central focus of nursing has not been strongly emphasised. While health has been acknowledged by nurse theorists as an important concept in nursing and in their theories, the emphasis in teaching has been on the narrower focus of assisting people deal with factors that influence their health, factors that hinder their potential and restrict their participation in society. For nurses, the emphasis on action, intervention or therapeutic processes of care delivery has narrowed the scope of their practice. Therapeutics such as touch, care, role supplementation, protection, comfort and use of self have emphasised nursing as a process and an interaction, rather than on nursing as an agent to co-ordinate the achievement of an outcome. The domain of nursing is articulated as dealing with patients or clients who interact with their environment, who have unmet needs and are unable to care for themselves or are not adapting to their environments due to interruptions in health (Meleis, 
1991, p.112). The domain of nursing in the broad concept of health may be being taught at under graduate level but the practice of reality is that few can work on those factors that impact on the health of individuals and communities, to maintain social productivity and health, and minimise illhealth. Once socialised into inpatient treatment and recovery settings, the perspective of the nurse narrows.

Nursing fits into the whole of healthcare as an integral component of the interdisciplinary team. Each discipline makes a unique contribution to the promotion and preservation of health and the prevention of health problems. If nursing has a narrow perspective about its place in supporting health outcomes, the profession restricts its potential to influence and contribute into the future alongside the healthcare team.

Chopoorian (1986) and Moccia (1988) have been studied in this section of the chapter because of their strong challenge to the nursing profession to consider the nursing role as a social entrepreneur rather than a passive provider of service under direction. These authors provide strong professional recommendation to look at health in the broadest way. The concerns they raise remain relevant as the profession finds its place in the health service of the twenty-first century. Their challenge creates a mandate for nursing action, if the profession is sufficiently sensitised to their broader responsibilities. 
Has nursing lost influence because of the narrowness of its perspective and its difficulty in measuring health outcome? In some respects this could be answered in the affirmative. The diversity of practice, practice specialisation and the treatment-recovery focus has hidden the impact of nursing on health gain. Non-nursing roles have evolved to monitor the environment, to promote health programmes, while nursing activity in primary health in New Zealand has been narrowed by managers and service provision contracts, to focus on age-specific health initiatives rather than the whole family or environmental health. This may have arisen because the majority of nursing roles have been focused on in-patient activities and on the "immediacy of individual concerns rather than larger social, political and economic concerns" (Chopoorian, 1986, p.46).

If nursing is to achieve its full potential as an agent of health change, then nurses need to develop knowledge and confidence to take the initiative in advocacy for social change and to seek work in these fields. This not always easy where employment specifications are restrictive. In recent years there has been increased expectation from health planners (National Health Committee, 1998) that nursing, as one of the major health provider professions, would want to work more independently as autonomous providers to meet the health needs of communities, especially those with low health status. Only a relatively few nurses have negotiated contracts as autonomous providers. While a group of nurses are employed to improve, promote and protect public health, the majority of nurses are employed in services where the focus is on maintaining and restoring the health of people 
who are normally healthy, to maintain or improve health and independence and increase the quality of life for people with chronic illness or disabilities. While all aspects of nursing practice in some way act to restore health, nurses also have a responsibility to know and influence those areas that impact on health "the conditions that control, influence and produce health or illness in human beings" (Chopoorian, 1986, p.53). If nurses are to "enhance the abilities of individuals and groups to achieve their health potential within the realities of their life situations" (New Zealand Nurses Organisation, 1993, p. 31), then they must understand the determinants of health. They cannot be effective in planning with people how they can maintain health within their personal reality without this broader perspective.

Acceptance that all of nursing in some way promotes health in its broadest sense is not a new phenomenon. In 1945, nurses in New Zealand were challenged to influence the health of the nation, described thus:

a nation's strength is decided by the physical and mental stamina of its people, military and civilian, and the maintenance of that stamina at a high level is, to a considerable extent, the responsibility of the nursing profession (Kai Tiaki, 1945, June, p.1).

The profession in the 1940s claimed that every branch of nursing contributes, whether by curative care or health teaching and all are equally important. Nurses because of their calling and because of their citizenship, have a double interest in the schemes for post-war reconstruction. Now more than ever before, it is 
acknowledged that good health is the first and most important asset of any nation. Without good health, progress in other directions must necessarily be slow and sporadic. The health of the community is the concern of the nursing profession and the plans for post-war living which have more directly to do with health are likewise the concern of all nurse (Kai Tiaki, 1943, June, p.1).

These references are cited as evidence that nursing in the twentieth century did have a focus on health in its broadest sense.

There continues to be a place for nursing involvement across the whole spectrum of health service provision. The challenge is to link current practice initiatives and demonstrate where the most health gain can be achieved. It is essential that the profession provide evidence that "the standard of health in a community is determined to no inconsiderable degree by the efficiency and extent of the nursing service" (Kai Tiaki, 1943, June, p.1) and to reinforce the outcomes that nursing achieves through effective research measuring economic benefit. This remains true now as it was in 1945. Nursing has an integral place in promotion of health, prevention of disease and facilitation of health recovery. To be effective and to influence change, nursing needs to regain a holistic perspective about health and determinants of health and to seek ways to promote health to individuals and communities of influence. This may require a reframing for the profession but is essential if nursing is to remain a viable and influential profession with a voice of advocacy. 


\section{Health service priorities}

Public or population health is considered the science and art of preventing disease, prolonging life and promoting health through organised efforts of society. This definition is broadly inclusive of all of the factors and action that impact on disease prevention, prolonging life and promoting health. This broad perspective has remained a constant focus of society in New Zealand even as new knowledge, technology and scientific developments have emerged, such as genetic, biological, behavioural, socio-economic, cultural and environmental initiatives. Public health programmes, interventions, policies and activities have sought to improve and protect the health of individuals and the community in medical, educational, promotional, legislative, regulatory and economic areas (Public Health Commission, 1995; National Health Committee, 1998). Since early settlement, the organised effort in public health has consistently targeted prevention of disease, prolongation of life and promotion of health.

While public health was considered important, it has been consistently underresourced in comparison to personal health. Regardless, the greatest improvements in health over the past century, particularly in the decline in infectious diseases, were improved public water supplies and sewerage disposal, nutrition and general living and working conditions as well as specific population health interventions (Rice, 1991). Yet expenditure on hospitals as treatment centres and in the use of medicines has been high in comparison to that spent on other areas impacting on health. Recent population health interventions such as fluoridation of water supplies, 
tobacco control measures and immunisation have been generally effective although public resistance to general interventions such as immunisation has remained.

Improvement to general population health continues to receive priority attention, with particular emphasis placed on reducing health inequalities that impact on the Maori population. Improvement, however, has been slower national and international health reforms have focused more in the past two decades on the structure of the health service delivery, on the curative and hospital care aspects, seeking universal access to health services rather than on health promotion itself (National Health Committee, 1998, p.58).

Recent developments in genomics and emerging genetic research and technologies, promise to enhance the capacity for early detection and preventative action to reduce the risk for disease, to act on environmental determinants and the moderation of behaviour. Health planners have identified that in order to eradicate disease, each country needs to develop appropriate health systems that: have a clear health policy and strategic plan; organise health systems, structures and processes to achieve targets; mobilise and use financial resources effectively; ensure adequate number and quality of human resources; and ensure that routine health services are capable of reaching under-served communities using information/surveillance-based service delivery (World Health Organisation, 1998). 
Despite decreased public funding, an increasingly educated and informed population has demanded that health services increase their access to new technologies to address their needs. This new technology is expensive and is not able to be paid for by all governments. With this in mind, the World Health Organisation (also referred to as the WHO) has encouraged sustainable health systems that support a longer-term vision, a statement of explicit values, the forming of strategic partnerships and integration of different efforts across regions rather than isolated action. The WHO considers that the greatest health gain for people is achieved through five long-term priorities, namely: promoting social responsibility for health; increasing investments in health to reduce inequities; consolidating and expanding partnerships for health; increasing community capacity and empowering the individual by ensuring human rights; and securing an infrastructure for health promotion. These approaches focus on primary health and determinants of health at their most basic, rather than emphasising interventions that may be beyond what many governments can provide. The emphasis is 'health for all' not just for those who can pay.

It is international experience that overall life expectancy is lower in countries with wide income differentials, regardless of how much of gross national product is spent on health (Smith, 1991). There is, however, no evidence that simply relying on an increase in the overall wealth will reduce the numbers of people experiencing poverty or will diminish the gap in health. This then supports the recommendation of health planners to target where the most gain can be achieved. Essential prerequisites for achieving 
health targets have been identified as: peace and freedom from fear of war; equal opportunities for all; the satisfaction of basic needs; adequate food and income; basic education; safe water and sanitation; decent housing; secure work and a satisfying role in society (Ministry of Health, 1999a, p.215).

In New Zealand these simple targets can be achieved where nursing works in co-operation with the wider social agencies and communities to gain commitment to address the determinants of health. There are examples where nurses work as co-ordinators of community development programmes to address the needs of people in the wider health determinant context. An example is the South Auckland District Health Board 'HARPIP' initiative. This programme (Hospital Admission Readmission Prevention Intervention Programme) works with the nurse as a central co-ordinator, to assess and coordinate the plan that best meets the health needs of individuals and families who re-present to the Emergency Room with acute illness exacerbation. There is evidence that better housing, consistent income and better social support can improve the health status of the individual and family and thereby minimise ill health. Income Support and Housing New Zealand assist the needs of these people with home improvements and employment or benefit allocation.

In order to develop evidence-based policy for health, national burden of disease studies are being strongly encouraged and supported by the World Health Organisation. The results of a 1999-2000 study, titled the Burden of Disease and Injury in New Zealand (2001), has been used to assist policy 
makers and health planners select and prioritise health objectives for the New Zealand Health Strategy (Ministry of Health, 2000c). Application of integrated measures of health to policy analysis can allow for strategic, funding and service delivery decision-making (such as health-adjusted life expectancy, disability adjusted to life expectancy or life year and quality adjusted). There are a number of ethical and technical difficulties to be debated and resolved as some consider the concept of "quality adjusting survival' problematic. In reality, trade-offs of quality for quality of life is inevitable under any policy setting, whether done implicitly or explicitly. The benefit of these measures allows policy makers to make value-judgements that are explicit and transparent about what health programmes will make the most difference and where resources are best spent (Ministry of Health, 2001, p.31).

Using a burden of disease and injury problem approach ensures that robust methods of analysis can be used to show the link of policy to data. Planners undertaking economic analysis of policies and programmes use health gap indicators, such as 'years lost to premature mortality', 'years lost to disability', 'incidence' and 'duration', 'disability weights', 'discounting', age weighting, co-disability and risk factor analysis to justify health spending priorities. They consider health expectancy indicators, so that social preferences can be taken into account, so that causal analysis can consider proximal causes and population attributable risks can be analysed. This is important from an economic justification basis. 
Health planners have consistently focused on health priorities that were by necessity clear, selective, compatible, achievable, balanced, quantifiable and ethical, as found in New Zealand literature from the period 1880 to 1945. They had little choice but to focus on areas where greatest impact could be achieved because of limited funds, developing options for treatment, cultural diversity and community resistance to centralised control. It is the joint efforts of these proactive and reactive activities that have reduced the incidence of infectious diseases and mortality. While epidemics and war have deflected resources and altered priorities, New Zealand has attempted to address the determinants of health in order to achieve health gain. The principles of the 'welfare state' have ensured that the core services have been accessible to all members of society regardless of their ability to pay. For Maori, however, there remains a significant difference in health outcomes and these targets continue to challenge health planners.

The political view is promoted that "public health policies and programmes are a long-term investment in better health for New Zealanders, that ultimately may reduce the need for health care, including hospital services" (Ministry of Health, 1998b, p.1). Encouraging people to take a more intelligent interest in their own health and well-being has been the priority of health planners through the century. Promotion of health has been a priority especially as the prevailing view has been that New Zealand is a healthy country. "It is wrong that a country so favoured as New Zealand should have a steadily increasing sickness and hospital bed rate and that so much of the 
public money should have to be spent on curing disease" (Kai Tiaki, 1943, March, p.1).

Government health priorities have remained similar to those of the past decades and provide a useful focus to explain why the Government is funding the programmes it does. The current Minister of Health has proposed that good health outcomes can be achieved through targeted action on the ten health goals. The goals include emphasis on: a healthy social environment; reducing inequalities in health status; addressing Maori development in health; a healthy physical environment; healthy communities, families and individuals; healthy lifestyles; better mental health; better physical health; injury prevention, accessible and appropriate health care services (Ministry of Health, 2000c, p.5). These goals reflect a similar emphasis to that throughout the past century.

In conclusion, good health and wellbeing for all New Zealanders throughout their lives is a current and past priority for political leaders and health planners. The government, in representing society, has acknowledged that good health and wellbeing rely on a range of factors, many outside the direct influence of the health sector, including genetic inheritance, age, gender, ethnicity, income, education, employment, housing, sense of control over life circumstances and access to health care services. While there is evidence of the changing needs of the nation over time, there is a constancy about the requirement for skilled workers to provide service in inpatient facilities, in community settings, leading projects for health promotion and addressing programmes to contain and eliminate diseases. The context changes, the 
range and specialty of expectations might extend but requirement for expertise remains.

However one defines health and however broadly the activities to restore and maintain health are described, nurses have been present. The issues and priorities have remained relatively constant, initiatives influenced by social and political events and the values of the time. Over time the Ministry of Health has demonstrated a leadership role in maximising the health of New Zealander through balancing the decisions about expenditure, the programmes promoting health. Through their accountability they have contributed to the economic and social wellbeing of the nation (Ministry of Health, 1995). Nursing has also been a significant contributor to the wide range of health services provided through this time. Nursing has been present in roles and has undertaken functions across the continuum of care and service provision described earlier. Nursing has been present in all activities from health promotion, communicable disease control, health recovery and symptom management to maintenance of quality of life. The work of nursing occurs with the aim of promoting good health to enable people to participate more fully in society and contribute to community productivity by pursuing their goals in life.

In the next three chapters the work of nursing in health will be discussed in more detail and the 'Contribution Model' applied to three case studies to show how the framework can articulate nursing contribution. The context within which nursing works forms an essential backdrop against which nursing contribution is profiled. The next chapter considers the practice of 
nursing historically and draws from the progress of health discussed in this chapter to locate nursing in actions that respond to social need and expectations.

In the three chapters that follow, the 'Contribution Model' is applied in the context of health service delivery, considering nursing contribution to health care using three periods: historical, contemporary practice roles and future functions. Health care practised by nurses in many contexts is the constant theme throughout this thesis. Nursing, whether in a complementary or 'situation-responsive' model or a replacement or 'a priori' model has made (makes) a contribution to the health of the nation and it is the articulation of this that has stimulated the development of the 'Contribution Model'. 


\section{Chapter Five}

\section{Nursing contribution over time}

"To know the past is to understand the present and to have hope for changing the future" (Jones, 1998, p.xxi). This philosophy reflects that of the Maori people, tangata whenua, who acknowledge that who they are as a people in the present, is influenced by the past and those people who have gone before. This perspective influences our society in New Zealand, ceremonially and practically. History influences our current reality and our readiness to respond to the future.

The contribution of nursing to the health of New Zealand over the past century exists in the consciousness of society through narrative, myth and memory. These influences remain within the consciousness of the profession despite changes in education, practice, professional boundaries and technology. In this chapter, the 'Contribution Model' is applied using past historical events to create the context within which we can view the work of nursing reflected against the dimensions of society and health issues through these time periods. It is important also to understand the past and to use that to show how nursing has added value over time and how it will continue to do so because of its responsiveness to community needs.

\section{Method}

This chapter should not be viewed as a definitive historical work. Events from history have been used to create a case study using best available 
information that could be found from that time. The events happening in history and in health have been used as a two dimensional backdrop against which nursing actions, in response to events, can be profiled. While attempts have been made to comply with conventional historical research method, the details in this chapter are not meant to provide new insights into history, but to profile nursing contribution. Three time periods were chosen because they provide an interesting explanation of the influences on health service establishment in New Zealand and clear examples of nursing contribution. The early decades of European settlement in New Zealand were challenging times for nursing and contrast well with the lack of organised services prior to the introduction of modern nursing. The three time periods are: 1860 to 1900, 1901 to 1920,1921 to 1945 . I have divided the times in that way because considerable activity occurred before 1900 with the appointment of nurse leaders in hospitals across New Zealand and the establishment of nursing education programmes. This was followed by a period of changes in specific nursing functions as the poverty and isolation of rural communities was understood and community backblock nursing was introduced, to be followed by the impact of World War 1 and the influenza epidemic. The final period covers the economic Depression and major changes to social welfare and the occurrence of World War Two. These are significant periods in the history of nursing in New Zealand as a small country with a developing profession struggling to cope with expectations and need. The decisions of this period still have an impact on health services, as New Zealand is a young country and has always been vulnerable to the impact of global events. 


\section{Use of case studies}

In order to describe the place and contribution of nursing over time, a case study methodology has been used. This method allows a description of the context and events occurring in society and the health issues to which nursing actions respond. The case study method is well established in the qualitative research tradition and as a methodology it is responsive to questions of 'how' or 'why', emphasising detailed contextual analysis of a limited number of events or conditions and their relationships. The case study research method is described as "an empirical inquiry that investigates a contemporary phenomenon within its real-life context when the boundaries between phenomenon and context are not clearly evident; and in which multiple sources of evidence are used" (Yin, 1984, p.23). Case study method has also been described as the collection, exploration, description and presentation of detailed information about a particular participant or small group, in order to draw conclusions only about that participant or group and only in that specific context. It can also be considered a method of learning about a complex instance through extensive description and contextual analysis.

\section{Table 2. Applications of a case study model}

- an explanation of the complex causal links in real-life interventions, previously unknown relationships and variables can emerge from case studies and can lead to a rethinking of the phenomenon (Stake, 1981)

- to describe the real-life context, as an adaptive research structure, in which the intervention has occurred as a contemporary issue of people in the real world, particularistic in that it focuses on a particular system, situation or event (Merriam, 1988) 
- to describe the intervention itself, complete and literal description of the phenomenon under study; and heuristic to mean the discovery of new meaning.

- to explore those situations in which the intervention being evaluated has no clear set of outcomes

- to test a hypothesis to modify an existing theory.

Critics of the case study method believe that a small number of cases do not grounds for establishing reliability or generality of findings. In the context of this thesis, however, the reliability and generality of the case study itself is not the focus of the case study although accuracy is and essential requirement. The purpose of the case study is to show how the 'Contribution Model' and framework can work to communicate the contribution of nursing to health gain using the backdrop of historical events and literature that refers to nursing.

In this chapter, there is reliance on literature rather than observational study of specific situations and time periods. The focus of the case studies used in this thesis are the actions of nursing in the work with health, intricately connected to political, social, historical and personal issues. This surfaces a wide range of questions and adding complexity to the case study. The question is persistently asked: 'how nursing as a subsystem has contributed to health in a situation or time period?' While considerable information is available from multiple sources to explain the context, Yin suggests that case studies should not describe everything but should be selective in order to focus on answering the purpose of the study. For the purposes of this chapter, a descriptive case study method will be used, using a linear-analytic 
structure (Yin, 1994, p.138). The approach uses a simple data collection, data reduction, display and conclusion process.

The case study method proposes six steps including: determining and defining the research question; selecting the cases and determining data gathering and analysis techniques; preparing to collect the data; collecting data in the field; evaluating and analysing the data; and preparing the report. Figure 4 depicts the process flow.

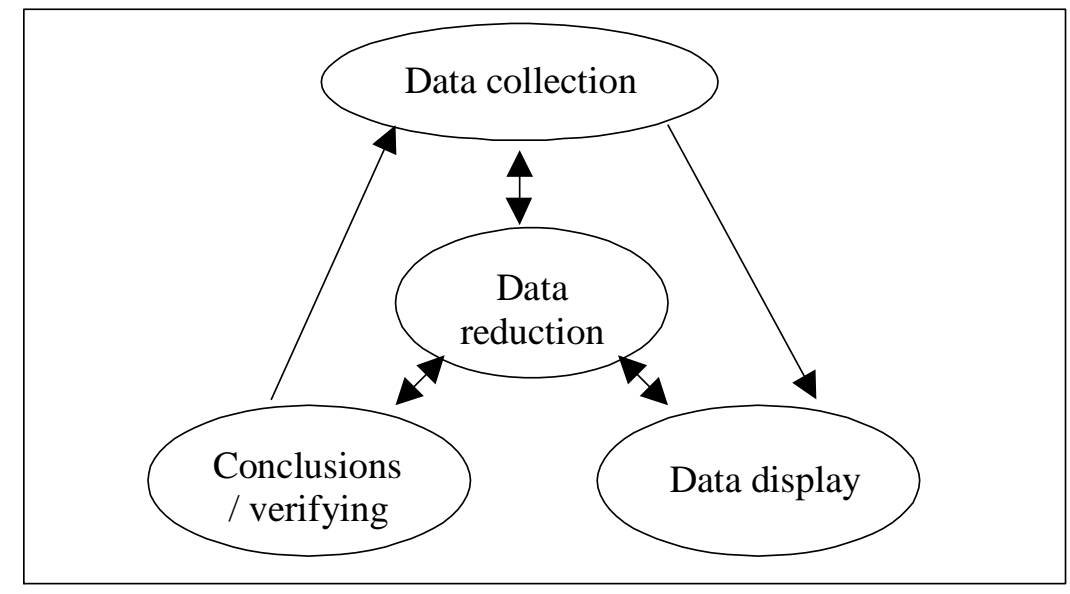

Figure 4. Case study method

Case study method is selected because this approach best suits the presentation of written material and historical reconstruction from existing literature. Case study and use of historical material assumes that although historical descriptions cannot always be proved true beyond all possibility of error, they can often be proved probably true, given empiricist assumptions. Assuming that the participants perceptions and data are very probably accurate, that their general knowledge and other beliefs are very probably true and that their forms of inference are 
generally reliable, one can rationally infer the probable truth of many historical descriptions (McCullagh, 1997, p.37).

Literature from the Department of Health annual reports, journals and biographies is used to create the case study. In this chapter, three case studies are outlined, using historical events in sequential time periods. The case studies are constructed in a systematic way. A general description is presented of main events occurring in society at that time period. Another general description is then presented about health issues that were prevalent at that time and reported in various literature. These two layers provide a snapshot and backdrop of what was happening in society and what was happening in health to a demand for nursing input. These descriptions attempt to show what directed nursing to take certain actions and become involved in different initiatives. The two initial layers may appear disjointed in places, as pieces of information are mentioned in apparent unrelatedness. They are included, however, to create a sense of the mores of the time period and to explain the priorities for service provision. They create a series of dimensions against which nursing actions can be profiled. This approach is used in all three chapters that follow.

\section{The case studies}

In the first case study, the conditions in the new colony are described and the introduction of an organised health service explained. It was not until the late 1800 s that nurses was actively recruited. This coincided with the availability of nurses from Britain who had trained under the newly 
established 'Nightingale' education programme that introduced modern nursing practice the hospitals of the 'Empire'.

\section{Case study 1. 1860 to 1900}

\section{Introduction}

"Comparatively little is known of Maori medical practices before European contact. Maori treated external wounds and ailments, such as warts, boils, toothache and menstrual pain, using herbal remedies. They were competent midwives. Physical and spiritual health was (and is) inextricably linked and were attributed too an attack by malevolent spirits on the 'mauri' or life essence. Mauri was normally protected by 'atua', but if the victim offended the 'atua' by transgression against the laws of 'tapu', then protection would be withdrawn and sickness would occur. The 'tohanga' treated mate Maori by recognising and treating the spiritual causes of ill-health by identifying the office and chanting the necessary 'karakia' to ward off its effects. While many Maori were interested to try 'pakeha' medicine, they returned to traditional practices because 'pakeha' medicine was less than efficacious" (Sargison, 1993, p.v, 2). The Maori were unprepared for the impact of death from the infectious disease that swept the country with the arrival of each wave of settlers. Many Maori people died during this period from exposure to infectious diseases against which they had little resistance (Pool, 1977).

In the early European settlement period, the conditions for new settlers was initially challenging. While the early settlers in 1840 had expected primitive conditions, later settlers had expected ready built homes as promised by the New Zealand Company who recruited some settlers for parts of New 
Zealand. Settlers had travelled for many weeks by sea. They had understood from the New Zealand Company they were coming to a settled new country. What settlers found on arrival was largely some constructed homes, land, tents and mud. Living was plain subsistence and many remained on the boat until such time as the men had building homes (Simpson, 1997). A number of settlers went inland to clear land for farming, or to work with an experienced farmer to learn the business of run holding. The initial period of settlement for many families was difficult.

Health care was not formally organised and the population generally relied on their own initiative and resources as well as the few health professionals (Dow, 1991, p.52). "The establishment of the Department of Public Health in 1901 formalised the state's role in the provision of health care, a role which would ultimately lead to the Social Security Act of 1938 and free medical care for all" (Sargison, 1993, p. vi).

\section{The socio-political context}

Population: In the pre-European period, the Maori population was estimated to be $200,000-300,000$ (Hyslop et al, 1983, p.1). By the end of the nineteenth century the Maori population was estimated to have dropped to 42,000 due to deaths from infectious diseases.

By 1842 Auckland and Wellington, the two main North Island towns were recorded as having populations of 3,538 and 3,901 European people respectively, which accounted for almost ninety percent of North Island pakeha (refers to European people). By 1851 Auckland recorded a population of 9,000 and Wellington 6,400 Europeans, which equated to seventy percent 
of the North Island pakeha population, with more people settling in rural settings and small towns in rural communities to establish farms. Between 1870 and 1885 the non-Maori population of New Zealand increased from 248,400 to 575,226 . More than 200,000 people entered New Zealand as immigrants between 1870 and 1880 (New Zealand Official Yearbook, 1899).

Immigration to New Zealand occurred in three stages. The first period in which significant immigration occurred was under the influence of the New Zealand Company and this ended in 1855. There was a period when immigration was assisted by provincial governments encouraged by the discovery of gold in some areas and this ended in 1870 . The third period of immigration covers the fifteen years to 1885 when central government assistance was provided to assist with public works and development (Rice, 1992). There were emigrants who were driven from their homeland by social circumstances and found in New Zealand an open society that allowed them to work, do well and realise their ambitions.

Records show that by 1861 about $35 \%$ of the New Zealand populations had been born in England; $28 \%$ was New Zealand born; $16 \%$ was from Scotland; 9\% from Ireland; $8.5 \%$ from Wales; $2.6 \%$ from Australia; and the balance from a wide range of other countries such as France, Netherlands and China (Simpson, 1997, p.8-9).

Life expectancy at birth in 1891 was recorded as 55.29 years for males and 58.09 years for females (Hyslop, 1983, p.48). The average infant mortality rate for European babies during the period 
1866 to 1876 inclusive was 103 per 1000 live births... Infant mortality was produced by diphtheria, scarlet fever, whooping cough, measles, fungal and bacterial infections of the gut and by poor ideas on infant care and feeding. The annual death rate for all ages in 1881 was 11.13 per 1000 of population. Which was half the rate of the United Kingdom. Of the deaths, 44 percent were in children under five years of age (Wright-St Clair, 1987, p. 17-18).

Education: The Education Act of 1877 provided a national system of free, secular and compulsory primary education for European children. Only 38 percent of pupils were girls in 1877. Secondary education for girls was provided by churches or small private schools that emphasised feminine accomplishments. Schools for Maori started from 1867, devised as agents for transmitting European values and practices into Maori life "to go forth to care for the sick, to lecture and to uplift humanity" (AJHR, 1898, H-22, p.12). The 1867 Native Schools Act decreed that instruction should be in English where possible and this was reinforced in 1890 by restricting the speaking of the Maori language in Native schools under the Native Schools Code. By 1886 there were twenty-two secondary schools in New Zealand: six for girls, eight for boys and eight for both sexes. (Coney, 1993, p.192).

Political tensions: Renewal of hostilities during the New Zealand Wars of the 1860s impaired relations between Maori and European. Maori leaders agreed in 1892 to form a kotahitanga or a union, which could present tribal and intertribal grievances to the Government and protect Maori rights under the 
Treaty of Waitangi. Twenty-one thousand persons signed the Kotahitanga pledge, rising to thirty-five thousand by 1895 (Dow, 1995).

A Liberal Government was elected under John Balance in 1891that proposed changes in social justice and social welfare, extending access to secondary education to all socio-economic classes by inaugurating the Free Place system and instituting technical schools (Coney, 1993, p.193; Munro, 1996, p.201). These political agendas were promoted in part by the community of immigrants who had experienced depressed circumstances through the depression from 1870 to the 1890 s and wished to ensure that the circumstances from their homelands were not repeated in the new society. Premier Balance's budget spelt out that the only safe policy for the colony was one of self-reliance, one that fostered colonial enterprise (Munro, 1996, p.201). The Liberal government was again returned to government in 1893 under the leadership of Richard Seddon.

Social issues: As early as 1854 concern was being expressed about "reduction of self-reliance of the people ... fostering the dependence on the public hurtful to the morals of a population" (Dow, 1991, p.52). By 1885 Grabham, the Inspector of Hospitals, alleged that "the provision of free hospital treatment by Central Government is pauperizing the population to an alarming extent and taking away inducements for thrift" (AJHR, 1885, H18B, p.1).

The New Zealand Womens Christian Temperance Union was formed in 1885 and through petitioning, lobbying and attempting to influence public opinion 
campaigned for wider social reform. They were concerned with alcohol use and lobbied to reduce the number of 'public houses' from 1 per 357 people in 1881 to one per 911 people by 1915 (Coney, 1993, p.128). Legislation in 1895 and 1910 forbade supply of alcohol to intoxicated Maori men or any Maori woman. These provisions were not lifted until 1945 with the passing of the Maori Social and Economic Advancement Act and the 1961 Sale of Liquor Act.

The Married Womens Property Act was passed in 1884, a revision of the 1860 Act, that enabled women to acquire, hold and dispose of property and earnings and to sue or be sued as femme sole. It gave divorced women control over their personal property and allowed deserted wives to keep their earnings. The Divorce Act of 1898 gave men and women the same grounds for divorce (Coney, 1993, p.177).

Ex-nuptial births in 1885 were reported at 4.5 percent of all live births (for Europeans). The Infant Life Protection Act was amended in 1896 because of concern for orphans and unwanted children. It arose from a social movement where children were viewed as investments in the future of the nation and Empire (Dow, 1995). The Act required that homes where children under four years old were being fostered should be licensed. The Act also required caregivers to be registered, to give details of the children and to submit to regular inspections. This amendment required that the mother's name be registered and the mother to fully breastfeed the baby for six weeks. Mother Suzanne Aubert opened a home with the Congregation of Our Lady of Compassion in 1892 with the aim of preventing infanticide and giving mothers a new start in life. She refused to disclose the names of the children 
in her care and received harsh criticism. Minnie Dean was hanged after being convicted of killing a baby in her care, the only woman ever to be hanged by law in New Zealand. The term 'baby farmer' referred to paid childcare workers (Munro, 1996, p.216).

The Society for the Protection of Women and Children was formed in Auckland in 1893 to prosecute in cases of excessive violence and to provide assistance to battered wives. The National Council of Women was formed in 1896 as a national feminist organisation. Women won the vote on 19 September 1893, "the first self-governing country to legislate for universal female franchise" (Coney, 1993, p.12). Elizabeth Yates was elected mayor of Onehunga in 1893, the first woman mayor elected in the British Empire and Ethel Benjamin was the first woman in New Zealand and the British Empire to qualify as a lawyer.

The Old Age Pension Act 1898 was introduced to redress the problem of increasing numbers of older men unable to care for themselves. This was not a universal pension but was subject to income levels and assessment of the length of time in New Zealand (Munro, 1996, p.251).

Employment: There was an international trade depression, from the late 1870s to the 1895 that resulted in a number of people unemployed or in lower paid employment. This ended in 1895 with the application of technology of refrigeration to agriculture, such as sheep meat and dairy produce, which enabled New Zealand to supply certain products internationally. 
The first labour legislation, the Employment of Females Act (1873), restricted women's hours in factories to eight hours a day although this was evaded by employers. Despite these protections, conditions for employees were poor. Factories were cold and badly lit, with no lunchrooms or lavatories. Poor working conditions were exposed at hearings of the Sweating Commission, established in 1890 to observe and receive submissions on cramped and poor conditions, excessive hours of work with few breaks, poor pay, withheld or no pay. The findings provided support for a raft of legislation by the Liberal Government between 1891 and 1894 to regulate labour conditions. The first women's union, the Tailoresses' Union was formed in 1889 with varied strength throughout the country. In 1890 , fifteen percent of all women were engaged in the paid workforce (Coney, 1993, p.211). A Department of Labour was established in 1892, a Factories Act was passed in 1891 and by 1894 restricted hours of work for women and children were in place and the Shops Act regulated conditions for shop assistants. The Industrial Conciliation and Arbitration Act was passed in 1894 and this imposed some rights for workers. Mrs Grace Neill was appointed the first female inspector of factories in 1894 and provided detailed reports of conditions in factories from her visits to all parts of the country.

\section{Health issues}


Early health issues facing the new colony included access to medical home based care and inpatient services to care for the increasing number of people arriving in New Zealand. Many of the settlers had little support except their immediate family members and if alone they relied on the good will of others. Care of the sick occurred predominantly in the home of the person (Dow, 1991), with visits from medical practitioners and local, often untrained, women who acted as midwives or provided nursing care to neighbours and others who had no family support. These lay women played an essential but invisible role in the healthcare of women and children for some years.

The types of health needs of the population ranged from the usual influenza, accidents, fever and infectious diseases such as scarlet fever, typhoid/enteric fever, septic and infective processes, diphtheria and smallpox. Other reported diseases and injuries included acute bronchitis, fractures, acute abdominal obstruction, tuberculosis, peritonitis, acute nephritis, pyelitis, cystitis, rheumatic fever, coma, thrombosis, empyema, pneumothorax, Brights disease and venereal diseases (Maclean, 1960; AJHR ${ }^{2}$, 1897, H-22; Kai Tiaki, 1908-1910). Between 1872 and 1886 the death rate among European infants from diarrhoeal diseases was 26.1 per 1000 but by 1900 it had reduced to 14.2 per 1000 and by 1950 was 0.3 per 1000 .

Death registration for the European population was compulsory from 1855 , although it was not until 1930 that reliable information was available about

\footnotetext{
${ }^{2}$ As in the previous chapter, AJHR refers to the annual report to the Government of the day by the Inspector of Hospitals of the Hospitals \& Charitable Institutions of the Colony, later Department of Health.
} 
the Maori population (Hyslop, 1983). A smallpox epidemic in 1872 caused a number of deaths in the Maori population. Measles outbreaks were reported in 1875,1877 and 1893 and again had considerable impact particularly on the Maori population (Journal of Department of Public Health, 1918; Maclean, 1960). Maori life expectancy was 40 years.

"In New Zealand there was surprise at the number of "lunatics" as they were called, although it was noted that many of them were related to a high consumption of alcohol. The lunatic asylums of the colony were in a disgraceful condition according to the reports of the inspector of lunatic asylums, Dr F.W.A. Skae" (Wright-St Clair, 1987, p. 16). The Lunatics Act of 1851 required all mentally disturbed patients to be committed to jail. In 1851 the first female lunatic asylum was erected in Auckland for eleven patients, with funds from public subscription. Other lunatic asylums were built in Auckland, Wellington, Hokitika, Dunedin and Christchurch in the 1860s.

Inpatient facilities:

The proposal to erect hospitals in the new colony was suggested by Dr J.P. Fitzgerald in 1840 to serve new immigrants and the native population. In 1846 Governor George Grey granted money to build hospitals in Auckland, Wellington, Wanganui and New Plymouth (Dow, 1991, p.45). Auckland Hospital opened in 1846 with Dr William Davies appointed as Colonial Surgeon. The New Plymouth and Wellington hospitals opened in 1847. Wanganui Hospital opened in 1851 with George Reeves appointed Colonial Surgeon and Native Medical Attendant in Wanganui. Dunedin Hospital 
opened in 1852 and Lyttleton Hospital in 1853. Land was purchased in 1859 in Christchurch for Hagley Park and the hospital. The hospital was completed in 1862 with Dr Courtney Nedwill appointed surgeon and Medical Officer of Health for Christchurch. By 1870 there were 19 hospitals including hospitals in Otago and the West Coast because of the growing population of gold-miners. There were 37 hospitals by 1882. Waikato Hospital was founded in 1887. The Inspector of Hospitals was reported to be concerned about the "heedless determination of the people of New Zealand to have hospitals at their doors in every centre of population" (Dow, 1991, p.48). Otaki Hospital opened in 1899, 80 kilometres north of Wellington. There was reportedly little female accommodation provided. Another sixteen hospitals were opened during the first decade of the twentieth century, from four hospitals meeting the needs for a community of 22,000 in 1851 . By 1910 there were 56 public hospitals caring for a population in excess of a million people (Dow, 1991).

Hospital facilities were initially under the direction of a master and matron, generally untrained in health care provision. Unqualified 'nurses' and male attendants were employed from the domestic class to provide basic environmental hygiene and they were assisted in the wards by convalescent patients (Brown, Masters \& Smith 1994, p.10). Conditions were described poor cooking, rough handling and intoxication a common feature. These first established "nursing services" in New Zealand followed the English pattern that Florence Nightingale had so strongly deplored (Board of Health, 1974, p.12). At Auckland Hospital a Matron was appointed to clean, cook, serve meals and to supervise the non-trained staff; there was minimal medical 
cover. Many people were reported to receive better care at home with a private nurse and a visiting doctor. In this period the need for an organised nursing services was identified by both European and Maori leaders (Dow, 1991, p.57). The provision of inpatient services did improve as will be discussed under nursing issues.

The average length of inpatient stay was reportedly 34.03 days. Average daily cost of inpatient care was 4 shillings. 7 1/2 d. to 11 s. 71/4d (AJHR, 1897, H-22, p.49). In 1896, 1,521 people died in hospital and this reduced to 810 in 1897.

Dr Duncan MacGregor, appointed as Inspector-General of Hospitals for the colony from 1886 to 1906, warned against "pauperising the community through an over-provision of services in hospitals and charitable aid institutions" (Dow, 1995, p.33). The aim of government support was to preserve the recipients self-respect and independence (Lambie, 1956, p.14). The geographical spread and size of the hospitals established by 1897 is outlined in Tables 3 and 4. 
Table 3. Hospitals in the North Island of New Zealand in 1897

\begin{tabular}{|c|c|c|c|}
\hline Whangarei, & 17 beds & Auckland, & 36 beds \\
\hline Thames, & 54 beds & Waipu, & Coromandel, 20 beds \\
\hline Waikato, & 64 beds & Rawene, & New Plymouth, 56 beds \\
\hline Mangonui, & 12 beds & Gisborne, & Stratford, \\
\hline Wairoa, & 12 beds & South Wairarapa, 14 bds & Palmerston North, 67 bds \\
\hline Patea, & 16 beds & Hawera, & Wanganui, \\
\hline Masterton, & 37 beds & Dannevirke, 15 beds & Naseby, \\
\hline Pahiatua, & 20 beds & Wellington, & Total $=1155$ beds \\
\hline
\end{tabular}

Table 4. Hospitals in the South Island of New Zealand in 1897

\begin{tabular}{|c|c|c|c|c|c|}
\hline Nelson, & 66 beds & Picton, & 24 beds & Asburton, & 37 beds \\
\hline Christchurch, & 116 beds & Dunedin, & 149 beds & Southland, & 62 beds \\
\hline Akaroa, & 8 beds & Timaru, & 74 beds & Waipawa, & 42 beds \\
\hline Waimate, & 33 beds & Grey River, & 60 beds & Wairau, & 44 beds \\
\hline Oamaru, & 46 beds & Wallace \& Fi & $1 \mathrm{bds}$ & $\begin{array}{l}\text { Northern } \\
\text { beds }\end{array}$ & Wairoa, 14 \\
\hline Otaki, & 17 beds & Westport, & 30 beds & Westland, & 47 beds \\
\hline Havelock, & 7 beds & Reefton, & 36 beds & Kumara, & 24 beds \\
\hline Lawrence, & 28 beds & Arrowtown, & 12 beds & Mercury Bay & 9 beds \\
\hline Wakatipu, & 22 beds & Cromwell, & 14 beds & Dunston, & 13 beds \\
\hline Ross, & 16 beds & Charlston, & 11 beds & Total $=1092$ & eds \\
\hline
\end{tabular}

AJHR, 1897, H-22, p.34

Health promotion activities:

"From the 1880 s the accusation of quackery and charlatanism was hanging over the purveyors of patent medicines and their customers were accused of gullibility" (Munro, 1996, p.200). Mother Suzanne Aubert, who was a nurse held in high regard with European and Maori alike, advertised medicines that she dispensed 'that were purely vegetable in origin, in no way hurtful in ... action' (Munro, 1996, p.200). Settlers and Maori relied on traditional health 
practices when unwell, seeking assistance when they had to but generally relying on family practices.

There was concern by government officials about the health of Maori as it was obvious that they were significantly impacted by infectious diseases to which they had not been previously exposed. In 1849 the publication in Maori language of 300 letters containing health advice on smallpox caused near panic amongst the Maori who had no experience of this disease (Dow, 1995, p.35). James Pope published Health for the Maori: A manual for use in Native Schools (in Maori and English) in 1884. The publication advised about vaccination, sanitation, burial customs and exercise and advised consumers to seek assistance of Pakeha medicine (McKegg, 1991, p55; Dow, 1995).

Health workforce: By the 1840s doctors outnumbered chemists by as much as thirteen to one (Belgrave, 1991). Qualified doctors were appointed as designated native medical attendants, although the calibre of men attracted to this work was not high (Dow, 1995). By 1897 there were 68 stipendiary medical staff employed, 188 trained nurses from English and New Zealand training programmes and 405 probationers in training (AJHR, 1897, H-22).

\section{Issues for nursing}

From 1877 hospitals were urged to employ female nurses as a prelude to essential hospital improvement reforms and this was gradually implemented. In 1883 Dr G.W Grabham, Inspector of Hospitals wrote a report exposing the conditions of neglect, apathy and abuse of finances as part of his report on the state of hospitals in the colony (Goodnow, 1935, p.32). Dr MacGregor, 
Inspector of Hospitals, suggested that trained nurses be employed to replace the "dirty, ignorant careless women, who brought death or ill health to many mothers and infants". He also expressed reservations about educating nurses, with "the very serious drawbacks which attach to a system of female nursing" (Dow, 1991, p.57).

The influence of organised nursing was increasingly noticed from 1874 as more formally educated nurses arrived in the colony. The number increased from only four women arriving on the Cathcart classified as nurses to ninetyseven nurses listed in 1884 among the new immigrants receiving assisted passage. Mary Lyons, the first trained nurse, arrived in 1879 to administer Masterton Hospital. Mrs Bernard Moore was appointed to Wellington Hospital in 1882. In 1883 Miss Annie Amelia Crisp arrived aboard the Westmeath to be Lady Superintendent of Auckland Hospital. All of these nurses were reported to have made marked improvements in the patient environment, improving the supervision of care as well as "reducing the wine and spirit bill" (Brown, Masters \& Smith, 1994, p.16).

In the 1894 inspection report by the Inspector of Hospitals, it was identified that excellent nursing was being demonstrated in Wellington and Auckland Hospitals under the direction of the 'Nightingale' trained nurses. The improved standard of nursing care and training of probationers was having a noticeable impact on the organisation and quality of care. Nurses were involved in "assisting at surgery, observing complicated procedures and administering ether as well as polishing the brass, sweeping the floors, 
cleaning the ablution block, dressing wounds, giving enemas and taking temperatures" (Dow, 1991, p.63).

Formal nursing education commenced in Wellington in 1883 and commenced in Auckland in 1888. Between 1874 and 1911 the number of those classified as nurses in the census increased from 259 to 2939 although there was some difference in definition of a nurse that was possible after the Nurses Registration Act was passed in 1901. It is reported that 811 worked as hospital nurses and 2019 worked in private practice (Belgrave, 1989, p.11, p.20). By 1895 a three-year training course had been instituted in Auckland, Wellington and Christchurch Hospitals, at the end of which nurses were examined in anatomy, physiology and nursing (Bennett, 1962). Education of nurses, however, was of lesser importance than achieving hospital operational efficiency. Nurses worked under strict militaristic rules, were trained to participate in doctor-directed treatments, were responsible not to the patient but to the patient's doctor. The hours of work were long, with probationers undertaking household duties as well as providing patient care (Belgrave, 1991, p.21).

Questions raised by the case study: How did nursing contribute to the health gain of New Zealand in this period of early European settlement to 1900? As described above, this was a period of early development when nursing was established as essential the organisation of health services that were still developing. 
To consider what nursing was doing to respond to the expectations and needs of the time period, the 'Contribution Model' will be used to structure the discussion. The 'Contribution Model' is outlined as a reference in Figure 5 below. The framework used to apply the model is outlined in Table 5 .

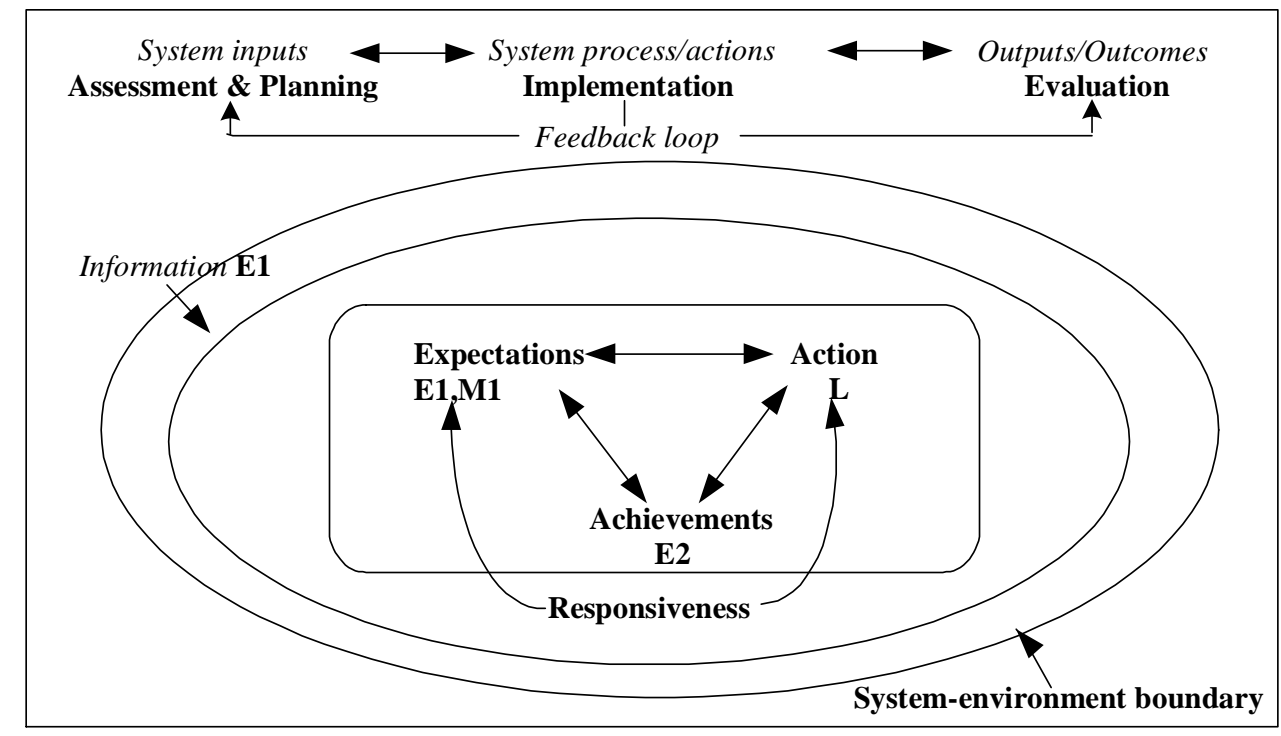

Figure 5. Complex adaptive system with nursing as a subsystem and the 'Contribution Model' depicted.

In the discussion above it appears that nursing, once established from 1870, introduced order from disorder, managed the environment, co-ordinated resources and personnel. Nurse leaders introduced standards of practice that included hygiene, care and behaviour and they provided a sense of consistency in the care provided. Nursing gathered information in order to assist with government decision-making. Nursing introduced nurse training programmes so that there was a process for ongoing self-sufficiency.

Using the framework tool developed from the 'Contribution Model', the actions of nursing in response to need and expectation can be profiled. The work of nursing is structured in Table 5 as follows. 
Table 5. Contribution framework for the period 1860-1900

\section{Expectations}

\section{Stakeholders}

Fewer people used hospitals in the early years but from the mid-1800s as more hospitals were built and nursing services improved, the use of inpatient care increased. What were their expectations of nursing?

Expectations of nurses by inpatients:

- Clean environment "utmost cleanliness prevailed"; "clean and in good order, food not wasted" (Scott, 1977, p.20; Brown, 1994, p.14).

- Good nursing care (Brown, 1996, p.14).

- Reliability, sobriety, good morals (Brown, 1994, p.11).

Expectations of nurses by community-based patients:

- Nurse able to go to their home (Brown, 1994, p.6; AHJR, 1912, H-31, p.4).

- Good nursing care (Brown, 1994, p.14).

- Able to assume control of situation (Brown, 1994, p.11).

- Trustworthiness (McKegg, 1991, p.84; Kai Tiaki, 1911, July, p.110).

- Sensitivity, care not to offend (McKegg, 1991, p.82; Kai Tiaki, 1911 July, p.108).

Expectations of nurses by decision makers:

- Good reports of standards of care - "excellent qualities of the hospital nurse" (Kai Tiaki, 1908, January, p.8). "Nursing in an extended sense, to include not only the actual personal attendance of the sick, but also the practical knowledge of hygienic principles and the use of simple drugs"'(AJHR, 1897, H-22, p.30).

- Organised services (AJHR, 1897, H-22, p.4).

- Educate the Maori on European methods for healthy living (McKegg, 1991, p86; AJHR, 1911, H-31, p.78; Kai Tiaki, 1911, July, p.108).

Expectations of nurses by decision makers:

- Cost management - replacing doctors with a cheaper and more effective network of district nurses (McKegg, 1991, p.61; Maori Affairs 21/20 N154. Valentine to Buddo, 29 June, 1909). 
Expectations of nurses by decision makers, continued

- Education and planning for more nurses to ensure adequate ongoing supply (Brown, 1994, p.18). "Attract intelligent educated women" (Scott, 1976, p.20).

- Information for reports and planning - "the eyes and ears of Hospital Boards" (AJHR, 1908, H-22, p.14).

Expectations of nurses by Medical practitioners (in-patient):

- Organisation and cleanliness (Brown, 1994, p.12).

- Sensible educated staff able to follow instructions, "intelligent and obedient (Brown, 1994, p.12).

Expectations of nurses by Medical practitioners (community-based):

Nurse able to go to the home of patients (Brown, 1994, p.12).

\section{Purpose}

The purpose of nursing at this time was

- to manage the health care environment and all resources efficiently

- to provide a wide range of physical care in a range of contexts in a safe and appropriate manner.

\section{Knowledge and skills required for the function}

A wide range of skills were required from these educated women: specific care delivery skills, "nursing in an extended sense, to include not only the actual personal attendance of the sick, but also the practical knowledge of hygienic principles and the use of simple drugs" (AJHR, 1897, H-22, p.30).

Organisational and good housekeeping skills were essential.

\section{Anticipated outcomes}

It could be presumed that the anticipated outcomes expected included:

- significant improvement in the standards of care and organisation "a very excellent type of nursing in operation" (Brown, 1994, p.15-16).

- improvement and consistency of practices (Brown, 1994, p.26).

- reliable back up for medical staff (Kai Tiaki, 1908, October, p.113; Brown, 1994, p.14).

- independent service by assessing what was required and alerting medical staff as required (Kai Tiaki, 1908, October, p.113; Brown, 1994, p.14)

- Maori nurses were identified as "fitted to be efficient preachers of the gospel of health" (AJHR, 1897, H-22, p.12). 


\section{Actions}

Nursing actions ranged from

- environmental cleaning and hygiene of patient care environments

- care of patients presenting with a range of physical and psychological disorders, including taking regular observations, administering medications, providing nutrition, wound care, comfort care, and encouragement.

- home care, district nursing care, nursing people through epidemics of measles, small pox, diphtheria.

\section{Achievements}

Nursing achievement in this period was obvious as evidenced by comments in annual reports about:

- Improvements in the organisation and standards of care that people in hospitals were receiving (AJHR, 1897, H-22).

- Control of costs where previously costs of alcohol and other resources use was considered high (Brown, 1996, p.5; AJHR, 1897, H-22).

Nurses worked alongside mostly unskilled workers as there were few with the formal nursing education. There was a high demand for more qualified nurses as more hospitals were built across the colony. Nursing achievement was evident in:

- Improved supervision of less skilled workers.

- Nursing education programmes in the main hospitals in order to recruit nurses to meet the growing demand for more expert care (Brown, 1994, p.15; AJHR, 1897, H-22).

The work of nurses in this period increased the trust and respect that the community had for nurses.

\section{Responsiveness}

Nurses could be said to have adapted to a new country as evidenced by their willingness to get involved in the hospitals where they were appointed and many stayed in positions for some time. Many nurses assumed leadership positions in hospitals across the country. They used their knowledge to establish standards of care for a wide range of health problems.

\section{How did nursing contribute to the health gain of New Zealand in this} period?

Nurses assumed responsibility for the management of the environment of the health treatment facilities and ensured that the quality of care was improved for those needing support to regain health. They raised the standard of health 
service provision so that the community could trust the services offered. Where previously care was erratic there were now increasing numbers of facilities where reliable and consistent care was provided under the supervision of trained nurses.

In this next case study, health services were more formally organised. Nurses were being asked to work in many areas of service delivery. Nurses wanted to go to war to support the New Zealand contingent in World War One. The government was reluctant as the country was already short of staff. A small number of nurses were dispatched for Europe. The influenza epidemic of 1918 posed a major challenge to health service and a number of nurses died during the epidemic.

\section{Case study 2: 1900 to 1920}

\section{Introduction}

During this period hospitals were better organised and the Department of Health, established in 1900, was providing stronger leadership on key issues.

\section{The socio-political context}

\section{Population:}

The population reached one million people by 1911, with the census indicating that the majority lived in towns. The 1916 census of Maori population, reported in 1919 , indicated that there were 25,933 males and 23,843 females (AJHR, 1919, H-31, p.13). Until 1912 the Maori population were exempted from the necessity of registration of births and deaths. Births of all groups from 1914 to 1918 were recorded as 961 in 1914, 976 in 1915, 
1031 in 1916, 1261 in 1917 and 1179 in 1918. Deaths for the same period were recorded as 765 in 1914, 817 in 1915, 808 in 1916, 773 in 1917 and an increase with 2306 in 1918, the increase attributed to the influenza epidemic (AJHR, 1919, H-31, p.13). The number of men over the age of sixty-five increased by fifty percent between 1896 and 1901, whereas the population as a whole grew by only ten percent. The ratio of those over sixty-five was sixty males to forty females. There were only marginally more married men than bachelors. Life expectancy in 1906 at birth for European males was 59.17 and for females 61.76 years (Hyslop, 1989, p.48). Maori women had a life expectancy of thirty years.

\section{Education:}

Only one percent of primary school leavers went on to secondary school in 1900 and only 45 percent of these were girls. Free places were introduced in secondary schools in 1903 (Coney, 1993, p.193). The Queen Victoria Maori Girls School opened in 1903 on the Native School estate in Glanville Terrace, Parnell. In 1913 "90 percent of children attending Native schools spoke only Maori at home" (Coney, 1993, p.196). By the 1950s, Hoani Waititi's study of Maori entrants at a number of secondary schools showed that only 26 percent spoke Maori, although 62 percent could understand it (Coney, 1993, p. 196)

\section{Political tensions:}

In 1900 New Zealand annexed the Cook Islands. New Zealand rejected federation with Australia in 1901 and became a Dominion in 1907. Women were entitled to stand for parliament from 1919. 
The Maori Councils Act was passed in 1900 and Maori local control was established. The Maori Councils were formed by statute and promised some measure of self-determination, but there were limits to their power. Maori sought to have a significant role in the political life of New Zealand. King Mahuta was appointed to the Legislative Council of the New Zealand Government in 1903. Maori leader Apirana Ngata was elected as Member of Parliament for Eastern Maori in 1905, Te Rangi Hiroa (Peter Buck) was elected for Northern Maori in 1909 and Maui Pomare was elected for Western Maori in 1911.

Tension arose between the government and Maori in Taranaki, over land acquisition and the death occurred of Te Whiti, the passivist resistance leader, at Parihaka in 1907, after troops were sent in to take control of the area. The Native Land Act of 1909 removed Maori titles and recognised marriage in accordance with Maori custom for the purposes of succession.

There was a strike by the Federation of Miners in 1908 that indicated the start of increasing industrial tensions between workers and the government. The Federation of Labour was formed in 1909 and had a significant impact on relationships with the government on behalf of the rights of workers. A strike at the Waihi mines in 1912 resulted in a miner being killed during a clash between the workers and the troops. Special constables were appointed to oversee developments. Wharf strikers clashed in Wellington in 1913 and fifteen hundred farmers occupied Auckland wharves to demonstrate against 
the actions of the wharf workers. The Court ruled that unions could not use funds to support a strike.

The passing of the Defence Act of 1909 made military training for boys compulsory over the age of twelve years. In 1914 New Zealand declared war on Germany in response to Germany's military action and declaration of war and New Zealand forces seized German Samoa. In 1915 New Zealand was part of the force that landed in Gallipoli and took Chunuk Bair. This event resulted in large casualties and death. Overall 2,721 men died during this action and 4,752 men were wounded. This was a significant number for New Zealand given the size of the country and the number of men sent overseas. The ship, Marquette, sent to transport troops and hospital staff, was sunk in 1915 and resulted in the largest loss of life of New Zealand nurses during World War 1 or any other military engagement. Ten nurses of the New Zealand Army Nursing Service and nineteen men of the New Zealand Medical Corps lost their lives among the 170 people who died. At home, a Wanganui crowd also attacked German-run shops to avenge the sinking of another ship, the Lusitania.

New Zealand troops entered the Western Front in 1916. Conscription was introduced and extended to Maori in 1917. Three thousand seven hundred more New Zealand men were killed at Messines in 1917. A German planted mine sank the ship Wimmera in New Zealand waters and twenty six people aboard drowned. New Zealand soldiers took Le Quesnoy as a last action in 1918 as the war ended. The Women's International League for Peace and Freedom was founded in 1916 and collected $£ 1,290,337$ to assist the 
repatriation of soldiers and their families on their return from war. New Zealand was one of the founding members of the League of Nations in 1919. From 1914 there was emphasis on post-war repatriation and rehabilitation.

The British Nationality and Status of Aliens Act was passed in 1914 (revised as the British Nationality and New Zealand Citizenship Act 1948) that required unnaturalised persons to register as 'aliens' with the police. These 'aliens' had to notify changes of address, occupation and marital status. This was used during the war to identify people of German origin.

Social issues: An increasing number of people needed social support from soup kitchens in the early 1900s as there were few social support systems for those without families and for the unemployed. In Wellington many of those needing assistance were known as “Wellington's workless, wet and weary wandering willies". Charitable aid was considered to be for children, not “cripples, drunkards and the diseased" (Munro, 1996, p.252).

Concern for women and children remained an issue and the Testators' Family Maintenance Act was passed in 1900 to force deserting husbands to pay maintenance. St Joseph's crèche or 'daylight home for babies' opened in Wellington in October for thirty-four children. The Home of Compassion in Wellington also opened in April (Munro, 1996, p.297). The Infant Life Protection Act was passed in 1907 and required notification of the birth of all babies within seventy-two hours. A widows pension was introduced in 1911. The Housing Act of 1919 allowed for low-cost housing loans to assist the reestablishment of soldiers after the war. 
There was a strong theme of morality was evident through this period. The Evidence Act was passed in 1908 that allowed for a 'breach of promise' action to be prosecuted; the Act was repealed in 1975. A Social Hygiene Act was passed in 1917 'for the protection of the health and morality of young persons' and this allowed for health patrols and also dealt with treatment of venereal disease. Te Oranga, at Burwood near Christchurch, was established in 1900 as the only girls reformatory in New Zealand. Girls were sent there on 'moral' grounds, rather than for criminal offences. This facility aimed at reshaping them into respectable and hardworking women (Coney, 1993, p.172). Barmaids were required to register, with nearly 1,300 women doing so. Hotel bars closed at six each evening as a wartime measure and it was not until 1919 that the returning soldiers prevented a move for the country to 'go dry'.

The Contagious Diseases Act had been passed in 1869, but was repealed in 1910. It allowed any woman suspected of being a prostitute to be summarily detained in a 'Lock Hospital', usually a prison. Not all women detained were prostitutes, but a number had difficulty proving this. The Obscene Publications Act was passed in 1910. The First World War, with its high venereal disease rate, raised the need for sex education that had been identified as necessary in the 1912 Cohen Commission on education. The Social Hygiene and Sex Regulation Society was formed in Christchurch in 1916 to provide books and pamphlets for women and referral to doctors for treatment. In 1918, 392 women visited the office and in 1919, 645 women and some men called (Coney, 1993, p. 178) 
Employment: In 1901 15,400 dwellings reported employing servants; 13,500 had only one, 1,590 had two, and only twenty had five or more. The census of 1901 showed that 25 percent of European storekeepers were women, mainly drapers, fruiterers, confectioners and grocers. Just over three percent of women were in the paid workforce and another thirteen percent were in business on their own but did not employ staff. The business women included: moneybroker / financiers (84), butchers (7), wine merchants (2), brewers (2), tanner (1), coal merchant (1) and (215) keepers of hotels and inns (Coney, 1993, p.218).

A Royal Commission was established in 1901 to look at staffing and salaries of public school teachers and a national salary scale was introduced the same year (Coney, 1993).

\section{Health Issues}

Inpatient facilities: While considerable care was still provided in the home with nursing and medical oversight, there was increasing demand for inpatient hospital care. The average occupied beds doubled from 1,290 beds in $1904-05$ to 2,131 beds by $1912-13$. This increased the bed population ratio from 1.51 to 2.04 beds per 1000 population. The first of seven St Helens Hospitals opened in Wellington in 1905 to provide training for midwives and to care for the wives of working men (Parkes, 1993). By 1907, there were 293 licensed private hospitals. 
There were still reportedly inadequate facilities for "incurable patients with cancer and tuberculosis, those who are old and feeble... and old brokendown miners" (Dow, 1995, p.51). The St Joseph's Home for the Incurables opened in January 1900, the first hospital of its kind (Munro, 1996, p.251). Interest arose in the establishment of centres for treatment of tuberculosis. Other sanatoria were opened for 'consumptive cases' at Flagstaff, Dunedin, Rock and Pillar in Central Otago. The Te Waikato Sanatorium, near Cambridge in the Waikato, was opened in 1903 (Dow, 1995, p.53).

There were 56 hospitals in 1910 (an increase of 3 hospitals from 1897) caring for 1 million people. A review by the New Zealand Department of Public Health reorganised the groupings of hospitals into larger districts, each with a base hospital, infectious diseases hospital, annexes for consumptive patients, mental wards, old people's homes, chronic ward, maternity ward, secondary hospitals and cottage hospitals (AJHR, 1920, H-31, p.14). This in effect created hospital boards with twenty groupings in the North Island and sixteen groupings in the South Island.

Health status data: There was an increase in deaths from an average of 10,500 in 1910 to 16,364 in 1918 , the majority of people in the 1918 numbers being aged between 20-45 years. The major causes of death are shown in Table 6 showed that a significant cause of death was respiratory and infectious diseases.

Table 6. Cause of death in 1918

\begin{tabular}{|l|l|l|l|}
\hline Infectious diseases & $20.5 \%$ & Heart disease & $9.1 \%$ \\
\hline Respiratory diseases & $13.7 \%$ & Accidents & $8 \%$ \\
\hline Cancer & $6 \%$ & Cerebrovascular accident & $5.1 \%$ \\
\hline Conditions of old age & $5.1 \%$ & Premature birth & $3.8 \%$ \\
\hline Other & $28.7 \%$ & & \\
\hline
\end{tabular}


(Hyslop, 1993, p.57).

One hundred and fifty-eight women died from complications of pregnancy and childbirth between 1916 and 1920 (Coney, 1993, p.60). Reported suicides were also noted with fourteen women were recorded as killing themselves in 1908, twenty-two in 1909 and fourteen in 1910 (Munro, 1996, p.314).

Health service organisation: A new Public Health Act became law on 13 October 1900 which effectively established the Department of Health. Joseph Ward was appointed as first Minister of Health with J Malcolm Mason the first Chief Health Officer. Te Rangi Hiroa (Peter Buck) was appointed as the Medical Officer for Maori Health. The Backblocks and Maori Nursing schemes were promoted by Maui Pomare and these became the cornerstone of rural nursing with the first nurse starting work on 14 August 1909. The St John Ambulance Nursing Guild was established and promised an alternative supply of district nurses for the chronically ill (Dow, 1995).

Epidemics, outbreaks, major health issues: A survey including all Australian colonies and most European countries from 1989 to 1908, showed New Zealand as having the lowest death rate both in infant and overall mortality. Deaths for children under one year of life, however, were still 74.2 per 1000, compared with 2.8 for ages one to nineteen years and 49.1 for sixty years upwards (AJHR, 1909, H-22).

The prevention and management of infectious disease outbreaks was continued to be a major priority during this period. There continued to be 
epidemics and outbreaks of measles and scarlet fever in 1907, scarlet fever and typhoid in 1908 and influenza in 1911 that had a major impact on the death rate, compounded by diarrhoea and gastro-enteritis (Munro, 1996, p.318). There was a smallpox outbreak affecting mainly Maori in 1913 and meningitis killed soldiers in the poorly equipped Trentham Camp in 1915. During 1920, diphtheria notifications were reported at 5,458, scarlet fever at 2,755 , poliomyelitis notifications were reported at 1,018 and tuberculosis at 1,521 (New Zealand Journal of Health \& Hospitals, 1921). Deaths from tuberculosis averaged 730 per annum from 1910 to 1919 , a rate of 6.87 / 10,000 (AJHR, H-31, 1920).

In 1918, the influenza epidemic claimed 8,000 lives. Maori were most affected at a rate of 226/10,000 (1,160 deaths) compared with 49.6/10,000 of Pakeha population (AJHR, 1919, H-31, p.13). Outbreaks had occurred previously in 1803, 1833, 1837, 1847 and 1890 (New Zealand Journal of Health \& Hospitals, 1921), but the 1918 outbreak had the most serious impact, coming as it did so soon after the end of the war. Many women with nursing backgrounds helped in the epidemic, as there were not enough nurses able to cope with the demands. A Royal Commission of Inquiry into the Influenza Epidemic was set up to assess action to be taken to prevent a recurrence of a disaster. It advocated sanitation and housing reform as some volunteers had been shocked by the conditions they found people living in. Women sanitary inspectors were appointed who could go the homes of the poorer classes, to talk to the wives in a kindly, simple way and to impress upon them the need for personal cleanliness for their own sakes and that of their children. There were also demands for more formal instruction of girls 
and women in home science as was being urged by the Plunket Society. The implication was that, as poor housewives, women had contributed to the conditions that led to the epidemic in the first place and encouraged improved home science teaching (Coney, 1993, p.91).

The epidemic of influenza occurred when there were 500 nurses overseas on active service. Many of the remaining nurses were ill and "quite threequarters of the total number of nursing staff being off duty together, either acutely ill or convalescing. The death toll among trained nurses and probationers as well as among doctors was heavy. The number of trained nurses available was not more that one to fifty or sixty patients. The large camps near Wellington were very heavily visited and the death tolls were large" (AJHR, 1919, H-31, p.10). Commendation was made to the voluntary helpers who assisted where there were inadequate numbers of health professionals. Afterwards nurses volunteered to offer training to the public under the guidance of the Women's National Reserve and the St Johns Ambulance Society, as many people had shown interest and aptitude.

Child health remained a concern, as despite relatively good statistics when compared with other countries, children were seen as essential to the future of the new country and their good health was viewed as important. Preservation of infant life became a priority and Premier Richard Seddon talked of saving these valuable lives (Monro, 1996). While Maori infant death rates were not recorded until the $1920 \mathrm{~s}$, it is estimated that the death rate could have been as high as 200 to $300 / 1000$ live births. Throughout the 1920s and 1930s the rate averaged between 90 to 150 deaths /1000 live births 
(Coney, 1993, p.64). Dr Frederic Truby King embarked on a campaign to promote the benefits of breastfeeding and developed 'humanised' milk for babies who had been weaned. A study undertaken had indicated that 25 of 1000 babies were bottle-fed in Dunedin (Coney, 1993). The Plunket Society was formed in 1907 at an inaugural meeting in Dunedin and was called the Royal New Zealand Society for the Health of Women and Children. The purpose was to encourage the correct feeding and care of babies and prevent "wastage of life" at a time when birth rates were falling and infant mortality was considered high (Sargison, 1993, p. 43). Lady Plunket had accepted patronage of the society, hence the name Plunket Society. Dr King provided his beach house at Karitane near Dunedin to help mothers establish good nutrition for delicate babies and be taught the essentials of infant care and parent craft skills. By late 1920 there were 64 branches of the Plunket Society and 500 sub-branches visited by nurses. Dr Truby King linked the care of babies to the health of the family, the nation and the Empire (Munro, 1996).

\section{$\underline{\text { Health promotion activities }}$}

Pomare published a handbook on infant care for Maori mothers on Plunket methods but modified the details to suit the needs of rural women. Called Ko Nga Tamariki me nga Kai ma Ratou, it had information in both Maori and English and was widely distributed by backblock and Maori health nurses (Coney, 1993).

The first Health Camp for children was organised by Dr Elizabeth Gunn at Turakina near Wanganui. The Sunlight League camps in the South Island 
also began in 1931 and introduced open-air schools to attend to the needs of children for fresh air, sunlight, good nutrition and dental care. The camps were standardised in 1936 when legislation was passed and a Federation of Health Camps was set up. A Dental School opened in Dunedin in 1907 as there was concern for the dental hygiene of children.

\section{Issues for nursing}

The status of nursing and formalisation of the social contract was acknowledged in 1901 when the Government approved the registration of nurses after strong advocacy from Grace Neill and Duncan McGregor, the Inspectors of Hospitals. The new regulations reinforced the requirement for nurses to be educated. Some politicians expressed concern that the "technical side of a nurse's training would 'spoil them as nurses' making them too mechanical and hard" (NZPD, 1901, p.390). Most politicians, however, were supportive of the registration of nurses "to raise the status of New Zealand's guardian angels, protecting them [society] from those who attempted to trade their well earned reputation" (NZPD, 1901, p.179).

This change in status was a threat to medical practitioners especially as nurses had been reported to criticise their professional conduct, such as nurses keeping a record of the doctor's attendance hours (Timaru 1899) and an inquiry into surgical standards was prompted by the complaint of a probationer (Auckland 1891).

In 1908 Dr Valentine, Chief Health Officer, expressed admiration of the many excellent qualities of the hospital nurse (Kai Tiaki, 1908, January, p.8) 
and bemoaned the difficulties of retaining nurses in hospital work with other employment opportunities luring the trained nurses from the hospital positions. Nursing literature through this period, in the newly established journal, Kai Tiaki, indicates increased interest in acquiring more knowledge about a wide range of health needs and increasing sophistication about treatment activities undertaken by nurses (Kai Tiaki, 1913, January, p.73).

While the increased hospital bed numbers provided employment and leadership opportunities for nurses working in the many hospitals across the country, women still tended to prefer home nursing. Probationers were paid more in country hospitals and this was proposed to allow them to save for transport to sit their exams in the four chief towns and the West Coast. Nurses were required in 1908 to pay $£ 1$ for the examination and registration. Training schools were only allowed in large public hospitals although private hospitals "of a certain standing" were considered able to provide adequate supervision. It was proposed to recruit as many women as possible to work as nurses, but this was tempered with the aspiration to also develop a strong international reputation for good professionals (AJHR, 1908, H-22, p.8). In 1908 it was also proposed to give "mental trained nurses who have undergone a course of lectures and passed the medico-psychological examination" a two-year course in general nursing. It was also proposed to offer a mental course as "a post-graduate course to general nurses to enter mental hospitals for a year" for experience in the care of the insane, to "fit them for positions in charge in mental hospitals" (AJHR, 1908, H-22, p.8). Twenty-five women were reported to have passed examinations to become "trained midwives", to add to the 1004 midwives on the Midwives Register, 
fifty-six having immigrated from England and Australia (AJHR, 1908, H-22, p.8).

Grace Neill, the senior nurse in the Department of Health, had clear expectations about the role of nursing in hospital services but was also concerned for the care of the sick in isolated communities and the need for health education of people. A community-based nurse for isolated communities was proposed in the 1909 Health Act although the initiative to have district nurses for Maori had been mooted as far back as December 1897 by the Te Aute College Students Association. McKegg reports that Hamiora Hei proposed,

I use the word nursing in an extended sense, to include not only the actual personal tendency of the sick, but also a practical knowledge of hygienic principles and the use of simple drugs (McKegg, 1991, p.62).

This had been acted on in 1898 in a scholarship system for Maori women (Wood, 1992).

Concern for the backblock families received attention. Families had been encouraged to establish farms and increase production for exports as refrigeration in the late nineteenth century allowed more intensive farming and transport of perishable food stuffs. Concern had been expressed after reports of women in rural areas giving birth unattended and the plight of sick children from isolated communities had alerted health planners to the needs of "women in the back-blocks" (AJHR, 1904, H-22, p.75; AJHR, 1909, H22, p.40; AJHR, 1914, H-31, p.7). Dr Valentine, supported the establishment of a "network of nurses all round the Colony; so that no back-block settler, 
and no settler's wife will be too far away to get help in their time of trouble (Kai Tiaki, 1910, April, p.28).

In order to establish a stronger community service, Grace Neill sought "women (nursing) of rare character, devoted to duty and undaunted by hardship", nurses with both nursing and midwifery certificates, with experience in country life and able to ride by horseback as roads were rarely passable (Kai Tiaki, 1909, January, p.4). The new scheme aimed to provide health care to people too poor, too distant or reluctant to seek professional medical services

to assist with illness until a doctors service could be procured... (to) visit the case and report to the nearest doctor if his services were required and to report daily the patients temperature, pulse, respiration and so forth so that the doctor might judge whether a visit would be necessary (Kai Tiaki, 1908, October, p.113).

There was difficulty recruiting sufficient numbers of nurses for this work, "a shortage of nurses for positions" (Kai Tiaki, 1912, October, p.99; AJHR, 1919, H-31, p.10).

The work was considered hard, with nurses reporting six to eighteen hours a day on the move in a two month period, almost lived in the saddle, riding from pa to pa watching the quarantine (Kai Tiaki, 1913, October, p.150); walking or on horseback, through mud and on isolated roads in all weathers (Kai Tiaki, 1909, July, p.105); 
the roads knee-deep in slush - you cannot walk anywhere (Kai Tiaki, 1911, July, p.109).

Nurses were expected to cover large areas of the country, travelling from eighteen to thirty miles a day; "covering 627 miles on horseback in six months" (Kai Tiaki, 1913, January, p.33). Nurses reported having to transport patients

for eight miles over a road so narrow only two people could fit. Six men volunteering to carry the patient... in relays... to where he could be conveyed by car (Kai Tiaki, 1911, April, p.74); and a woman transported to hospital using "a launch, wagon and car, "carried through the breakers to the launch (Kai Tiaki, 1921, April, p.198).

Most nurses remained in district nursing roles for relatively short periods of time. A study of Backblock and Maori health nurses (McKegg, 1991, p.184) identified that of the 109 women traced from 1909 to 1930,69 worked as Maori health nurses. Eighteen remained for one to two years, fifteen for three to five years and fifteen for five to ten years with sixteen working with Maori communities for ten to fifteen years. Of the 40 women who worked in rural European communities, ten remained less than one year, fifteen for one to two years, none for two to five years and ten remained five to ten years and two for fifteen to twenty years. These nurses left for many reasons including marriage, ill-health, accident, to nurse in World Ward One and three women died while in service from the very infectious diseases that were causing deaths in the communities they served. 
In June 1911 the care of the health of the Maori was transferred from the Native Department to the Health Department. Sending nurses to areas where infectious disease outbreaks were noted did not achieve "continuity and it was difficult to find suitable nurses for the work when they were wanted" (Kai Tiaki, 1911, January, p.20). The special nursing service was aimed at work among the 'Natives', not only to attend them in actual illness, but to send nurses to live among them, help them with advice and example to live a more hygienic life and to bring up their children in a healthy manner. The nurse would detect illness and seek medical aid for it... prevents spread of infection by isolating the patients or by getting them into the local hospital (AJHR, 1911, H-31, p.20).

Many of the nurses selected for this work not only promoted European health practices but were generally willing to compromise by using Maori methods where this seemed safe, but to also show the wisdom of European methods. Many nurses were only called to deliveries when problems arose as the Maori managed the process "pretty well" (Kai Tiaki, 1912, July, p.76).

The education of nurses in hospitals, for hospital practice, had not prepared them for the type of work required of them in isolated communities. Although aspects of home nursing practices, such as dietetics as had been taught as had fever nursing, there was an identified need for broader knowledge. "The district nurse must be even a better trained nurse than a hospital nurse, because she has so much less help at hand" (Kai Tiaki, 1911, April, p.76). 
A broad education was essential for nurses sent to isolated communities to establish fever camps. As one nurse commented,

to act as doctor, to diagnose, treat, prescribe and dispense, makes one sharpen every faculty to do the very best possible... I like the work and read more medical books now than ever before, in my nursing career (Kai Tiaki, 1913, April, p.73).

Nurses were not provided with many resources. For example, Nurse Akenei Hei was sent to a typhoid epidemic in 1909 with "vaseline, boracic powder, boracic ointment, thermometers, an enema syringe, throat spray and brushes, dressing forceps, carbolic soap, linseed, mustard and zinc ointment" (McKegg, 1991). Nurses were required to deal with wide ranging health problems and heavy workloads, in temporary fever camps with smallpox and typhoid (Kai Tiaki, 1914, January, p.48). Some described dealing with a mining accident "every other day" (Kai Tiaki, 1915, April, p.88), nursing "128 Maori, 19 in her cottage and 109 in their homes, 17 lectures to various groups and 4 school visits. Of the cases nursed 20 were of typhoid, 21 of pneumonia and 37 of influenza" in one year (McKegg, 1991, p.176).

Community nurses encountered a wide range of experiences such as impoverished social circumstances in the backblocks, one nurse reporting "such poverty I have never seen; it is most distressing to live in the midst of it" (Kai Tiaki, 1910, January, p.28). Nurses described some homes with no beds or blankets, and "little enough for the patient" (Kai Tiaki, 1913, March, p.113). These nurses attended normal maternity cases and worked with women, advising them during pregnancy and afterwards on the feeding of 
infants and children, recommending changes to the sanitary conditions of the kainga (village) (Kai Tiaki, 1911, July, p.108). These nurses undertook social work as well as the care of families with fever, injuries and other ailments. By 1914 there were "twelve district nurses working in the backblocks and country places... recognised as one of the most useful and satisfying means of carrying on their profession. In it they have more scope for individuality and initiative than in institutions or ordinary private nursing" (AJHR, 1914, H-31, p.7).

Hospital boards paid half of the nurse's salary and could determine the area where she worked. To make the scheme self-supporting the nurse was expected to charge fees, as guided by the Health Department schedule, with the nurse deciding the fee based on the family circumstances and reporting the decision to the Hospital Board (AKHR, 1914, H-31, p.14). Settlers were expected to assist the nurse in the case of emergency.

For many nurses the impoverished circumstances made enforcing payment difficult. District nurses were authorised to decide what they would do and whether they would charge for their services. The nurse was required to provide an explanation where services were provided free and were instructed to ensure that settlers were not discouraged from attending the medical practitioners because of free nursing services. By having access to a local nurse it was hoped to discourage settlers, in isolated regions, from setting up separate hospital districts because of dissatisfaction with lack of immediate hospital services. Some medical practitioners however were concerned that the money contributed by the local community to pay for half 
of the nurses salary and the costs charged by the nurse would limit their (the doctors) income. There was also concern that nurses presented a threat to medical care in the district, "the nurses work keeping the people in too good health for their practices (Ibid, 1922). Assurances were given that "in no sense of the word would the district nurse be expected to ... in any way take the place of the general medical practitioner (AJHR, 1909, H-22, p.6). Many nurses did not request money from families when they observed only one kerosene tin to carry water, one small tub to do all the washing and bathing in, a stove that smoked so badly that all were continually in tears... flooring boards so far apart that as one walked across them the black sharp eye of a hen could be seen peering though (Kai Tiaki, 1922, January, p.28).

Local committees were supportive of their nurse who became an important and significant person in the life and survival of local families and whanau.

Public health nurses were also involved in extensive health promotion and disease prevention education programmes. This included: healthy eating, to deal with malnutrition and the poor state of children's teeth; environmental hygiene including home cleaning, sanitation, food handling; and disease prevention, especially the infectious diseases including venereal diseases. Public Health Nurses were appointed as Public Vaccinators in order to promote vaccination (Kai Tiaki, 1914, January, p.46).

Dr Truby King established the training for Karitane nurses in breastfeeding techniques, discouraging artificial feeding, regimented feeding programmes, toileting and swaddling. Plunket nurses visited the homes in local 
communities to instruct mothers about the care for their babies. There was concern for busy farmers wives who were reported to wean their babies because they were so busy from " 4 a.m. and late to bed" (AJHR, 1914, H-31, p.8). Maori infant welfare, however, was not comprehensively addressed during this period. By 1914 there were twenty-seven nurses working in branches across the country as Plunket Nurses. While it was proposed that these nurses might take up some district nursing if under-utilised, this was not approved.

A New Zealand Army Nursing Reserve was established in 1914, attached to the Defence Department "to be called upon for duty when required". The Matron-in-Chief of the Department of Health was appointed as the leader of the Nursing Reserve as were the four Matrons in Wellington, Auckland, Canterbury and Otago. Their responsibility was to enrol a number of registered nurses (AJHR, 1914, H-31, p.8). There were too few nurses to supply the national hospital service requirement and there was reluctance to release a large number of nurses for Army service. Nurses, however, lobbied to be included in the Army Corps requesting that they be allowed to go to Europe during World War One to "to give nurses the opportunity of serving their country" (Kai Tiaki, 1915, January, p.1). More than 600 military nurses served in hospitals in Europe and the Middle East or on hospital ships in World War One. The ten nurses who died when the Marquette sank were part of the Number One New Zealand Stationary Hospital (Coney, 1993, p.304). Many military nurses did not return to hospital-based nursing at the end of the war, instead taking courses under the Repatriation Board in speciality practices such as massage to assist with rehabilitation of injured 
soldiers. Other nurses married and were not able to practise nursing as married women. Some nurses underwent a very thorough training in the administration of anaesthetics, with extensive experience in casualty, clearing stations and stationary hospitals. These nurses found it difficult to get positions on return as there were not roles for them in these areas. The Emergency Department nursing had not been started at that time.

Individual nurses were sent to Samoa to assist with new service development in the first decade of the 1900s.

There are limited written comments about community perceptions of nursing at this time although there are some notable indicators of satisfaction. Some impoverished communities made considerable effort to gather sufficient money to guarantee a sum of not less than half the salary and expenses of the nurse to be appointed and provided money in appreciation of the nurse's work (Kai Tiaki, 1911, September, p.130). The role of the nurse was apparently valued by the Maori; "many who were formally opposed to it have now expressed approval... and have extended much gratitude for nursing assistance" (AHJR, H-31, 1912, p.21). These nurses worked in communities with isolated settlers and Maori, many of whom did not have access to a hospital in the nearest town. A letter and gift of six pounds received from the Waima Maori people of the Hokianga in 1913 by the Chief Health Officer Dr Valintine, indicated their appreciation of the work of nursing:

we ask you to be assured that our love and gratitude towards them (nurses) is boundless... We have clearly seen that the only remedy in 
such times of danger [chicken pox epidemic] lies in the work of nurses (Kai Tiaki, 1913, July, p.151).

A report by Judge Jones of the Native Land Court also reinforced the respect with which nurses were held

a word of commendation of those brave and noble women sent out by the Department to assist in nursing the natives. Apart from the ordinary risks of their profession, they take their lives in their hands and put up with hardships and discomforts which only one who travels the same roads and crosses the same rivers can realise (Kai Tiaki, 1912, October, p.99).

This essential role was communicated to the Department of Health and their local politicians. Pomare wrote "these are the kinds of workers we need, for they get down to the people, and by practice demonstrate the beauties of a better and healthier life" (AJHR, 1907, H-22, p.54). Until more nurses became available, some communities were under-serviced and were required to share a health nurse. As more nurses were made available and the nurses learned about their role and the needs of their community, they were able to make progress and gain the confidence of those seeking assistance. Nurses worked to increase awareness of the scientific theories underlying the spread of disease and to increase the confidence of communities to seek assistance from the developing health services.

Nurses appeared to be increasingly aware of the issues of cultural sensitivity and their lack of special preparation for this aspect of their work. While not always culturally insensitive, many of the district-backblocks nurses had to 
learn what was culturally accepted or not. "Maori were very quick to perceive one's attitude towards them and that any slight would very quickly drive them away" (Kai Tiaki, 1914, January, p.48). Akenei Hei cautioned her nursing colleagues

Great discretion must be used not to offend the patients beliefs and at the same time uphold one' own mission ... such customs (ancestral), having kept the Maori race in vigorous health from many generations, deserve consideration... A greater knowledge of the native mind will inspire a greater and thereby a deeper sympathy for the Maori people... Who cares to have a stranger poking around his back door, condemning the hundred and one things which sanitation know are detrimental to public health? (Kai Tiaki, 1910, July, p.103).

Many of these nurses knew "nothing of the Maori customs, made many mistakes 'which must hurt them', they forgave and taught me a lot" (McKegg, 1990). Te Rangihiroa reported

The Maoris realise and appreciate the good work being done by the nurses and many districts are asking for nurses to be appointed... I consider the nursing branch of the Maori work the one that should be assisted and pushed on more than any others" (AJHR, 1919, H-31, p.11).

Nurses were expected to be agents of the state, portrayed as 'health missionaries', to "go forth to care for the sick, to lecture and to uplift humanity" (AJHR, 1908, H-22, p.119). They were to enforce community sanitation, address issues of dirt, dress, tohungaism, substance abuse, poor 
presentation for vaccination, impact of tuberculosis, and the high infant mortality rate. This role was not always well received by communities. Nurses only achieved acceptance where they demonstrated flexibility, understanding and empathy, a gentle and mutual sharing of knowledge and skills that allowed a mutual co-existence and respect (Kai Tiaki, 1911, July, p.110).

By health lectures and practical instructions the preventative part of their work is as important as the actual nursing of cases... general routine with regard to nursing, feeding and disposal of excreta (in epidemics) will prove of great educational value to the people (AJHR, 1919, H-31, p.11).

Alongside the early missionaries, nurses worked with the local women to assist Maori communities' cope with diseases that were having a devastating effect on the health of children, adolescents and the elderly. As front-line workers in rural settlements nurses coped as best they could with diseases and health problems in the absence of medical and hospital services (Burgess, 1984).

Nurses themselves perceived their value to be high, writing: "there is no doubt a good nurse is better than the average medical practitioner of the back-blocks" (Kai Tiaki, 1917, March, p.117). The success and influence of nursing was the ability to "sit down in the various districts and by precept and example, help their countrymen to a good and healthy way of living" (Maori Affairs, 1909, p.20-21). 
Nurses were given strict rules to follow

faithfully follow his [doctors] orders, keep him informed daily on the patient's condition, not to use their influence to promote one doctor over another, to guard against acting autonomously and only use their initiative in an emergency when medical direction was not available" (AJHR, 1911, H-31, p.77).

It is "unwise to undertake such work as it was the preserve of doctors" (AJHR, 1912, H-31, p. 69).

In these early settlement days, nursing was able to influence the direction of health service development through close association with the senior medical colleagues in the Department of Health and advocacy at local Hospital Board level. Mrs Grace Neill and Miss Hester MacLean, as assistant Inspectors of Hospitals, worked hard to ensure that nursing received the recognition and status they thought appropriate.

In 1914, the supply of nurses was boosted by reducing the age of entry from twenty-three to twenty one and retaining nurses for a 'fourth year' after training with inducements of post graduate courses. Topics such as "hospital management, dispensing, midwifery, massage and electrical treatment" (AJHR, 1914, H-31, p.6) were offered. Internal recruitment was essential to meet demand as very few 'foreign' nurses "find their way to New Zealand" (AJHR, 1914, H-31, p.6) so recruitment from overseas was not easily achieved. By 1917 the workforce supply issue had become dire although increased numbers of nurses were reported to pass the state examination, 
"1913-1914 being 191 and 1918-1919 being 288". The increase in demand was

necessitated by the return of sick and wounded men from active service... the policy being to send these men for treatment to the general hospitals of their own districts rather than to special military hospitals". "The senior sisters being constantly called up for military service have left gaps to be filled by nurses only recently qualified and without experience in teaching. The provision of nursing assistance in country districts has been a very acute difficulty" (AJHR, 1918, H-31, p.10).

Questions raised by the case study:

How did nursing contribute to the health gain of New Zealand in this turbulent period when demand for nurses was high because of war and post war rehabilitation, epidemics and general increase in inpatient beds.

Using the framework tool developed from the 'Contribution Model', the actions of nursing in response to need and expectation can be profiled. The work of nursing is structured in Table 7 as follows.

Table 7. Contribution framework for the period $1900-1920$

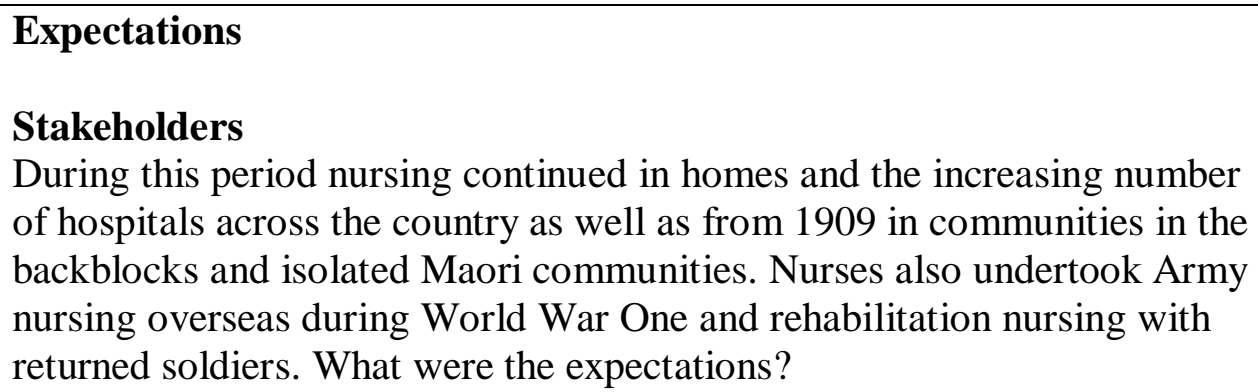
of hospitals across the country as well as from 1909 in communities in the backblocks and isolated Maori communities. Nurses also undertook Army nursing overseas during World War One and rehabilitation nursing with returned soldiers. What were the expectations? 
Expectations of nurses by patients (inpatient)

- High personal standards of conduct: "women of high calibre, jealously guard that position by checking the tendency to lower the professionalism in esteem of the public (Kai Tiaki, 1908 October, p.113).

- Good nursing care in hospitals and in army medical corp "practical nursing" (Kai Tiaki, 1914, July, p.124).

- "take off my hat... the position of the hospital nurse holds in the opinion of public, excellent qualities for the nature of work" (Kai Tiaki, 1908, January, p. 8).

- Adequate numbers of qualified nurses and midwives and probationers to meet the diverse needs of work in the general acute, maternity, private hospitals and sanatoria.

Expectations of nurses by patients (community-based).

- Nurse able to go to their home (Kai Tiaki, 1912, October, p.99).

- Good nursing care - "our love and gratitude towards them is boundless, we have clearly seen that the only remedy in such times of danger lies in the work of nurses" (Kai Tiaki, 1913, July, p.151; Kai Tiaki, 1908, October, p.113).

- Able to assume control of situation - "to assist with illness until a doctors service could be procured...so that the doctor might judge whether a visit would be necessary" (Kai Tiaki, 1908, October, p.113). "who in a very short time has full control in the most unobtrusive manner" (Kai Tiaki, 1910, March, p.58). "has direct effect in reducing the need for hospital admission and spread of infection by teaching sanitary conditions" (AJHR, 1914, H-31, p.8).

- Able to offer advice on all matters - "to act as a doctor, to diagnose, treat, prescribe and dispense, makes one sharpen every faculty to do the very best possible" (Kai Tiaki, 1913, January, p.73).

- Non-judgemental professional "for they get down to the people and by practice demonstrate the beauties of a better healthy life" (AJHR, 1907, H-22, p.54). “ Maori were very quick to perceive one's attitude towards then and that any slight would very quickly drive them away (Kai Tiaki, 1914, January, p.48). I "knew nothing of the Maori customs, made many mistakes which must have hurt them, they forgave and taught me a lot" (McKegg, 1990)

Expectations of nurses by decision makers

- Educated women able to cope with all clinical and social eventualities in a mature and appropriate manner.

- Women prepared to serve their community regardless of hardships "prototype of unselfishness, loyalty and devotion to duty... exerting herself to raise the standard of work to ensure uniformity... establish a code of ethics" (Kai Tiaki, 1908, October, p.112). "Undaunted by hardship", "put up with hardships and discomforts which only one who travels the same roads and crosses the same rivers can realise" (Kai Tiaki, 1909, January, p.4; Kai Tiaki, 1912, October, p.99). 
Expectations continued...

- Women who were confident practitioners yet prepared to defer to the medical practitioners.

- Satisfied communities.

- Adequate workforce supply of the highest standard to meet demand: "sufficient number of young women to train as nurses, the unsuitable weeded out" (AJHR, 1914, H-31, p.6). Valentine, "not attracting to its ranks the same stamp of women as was the case one or two decades ago" (Kai Tiaki, 1908, January, p.8).

- Good reports of the standard of care provided - "prototype of unselfishness, of loyalty and devotion to duty... exerting herself to raise the standard of work to ensure uniformity... establish a code of ethics (Kai Tiaki, 1908,October, p.112).

- Containment of disease (AJHR, 1918, H-31, p.10).

Expectations of nurses by medical practitioners

- Intelligent and educated nurses able to implement the care prescribed and call for assistance as required.

- Probationers supervised adequately.

- Nurses relied on able to travel wherever was required, regardless of weather conditions or isolation (Kai Tiaki, 1908, October).

- Educated women able to cope with all clinical and social eventualities in a mature and appropriate manner "a district nurse must be even better trained nurse that a hospital nurse because there is so much less at hand" (Kai Tiaki, 1911, April, p.76; Kai Tiaki, 1908, October, p.113).

- Women as confident practitioners and prepared to defer to the medical practitioners (Kai Tiaki, 1908, April, p.36).

- Not to compete with their practice or to provide too skilled (AJHR, 1922, H-31).

- Able to offer advice but not take over "nurses work keeping people in so good health for their (doctors) practices" R.Cameron to Dr Hughes, 2 October, 1922; Dr Hughes to Frengley, 6 October 1922). It is "unwise to undertake such work as it was the preserve of doctors" (Ibid, 1912). In no sense of the work would the district nurse be expected to ... in any way take the place of the general medical practitioner (AJHR, 1910, H-22, p.6).

\section{Purpose of the nursing role}

To provide a full range of accessible health care services regardless of complexity of need or presentation, including treatment, advice and education for individuals and families in towns and isolated communities using available resources and working in co-operation with the others health team members. 


\section{Knowledge, skills and expertise required}

Nurses needed:

- A wide clinical knowledge base to cope with the range of health presentations, for hospital and community care whether medical assistance was available.

- In-depth knowledge of infectious diseases, infection control, isolation techniques, common sense and initiative to cope with setting up fever camps dealing with new presentations, those dying, those recovering and convalescing.

- Knowledge and experience in the care management of people recovering from accidents or acute illness or dying.

- To be able to deliver babies, deal with childhood illness, cope with major lacerations and injuries without access to medical assistance.

- To be able to assess whether someone could remain at home or be transported with difficulty to a hospital some distance away, confidence to direct the transport process.

- To cope with people with mental illness in the community and those who did not comply where intervention was required.

- To be able to teach unskilled people and rely on their common sense

- To know how to ride a horse and eventually a car across primitive unsealed roads.

\section{Anticipated outcomes}

It was anticipated that the following outcomes would be achieved:

- Nurses would be present in all situations to facilitate community access to health care where previously it was not accessible.

- Nurses would manage infectious disease outbreaks with as little loss of life as possible.

- There would be safe delivery of babies and establishment of breastfeeding and mother craft.

- There would be improved environmental hygiene in communities with use of trenches and privies.

- There would be improved knowledge about nutrition and health practices.

- There would be improved child health through intervention in schools.

\section{Actions}

Nursing actions included:

- Care of patients in hospitals and in people's homes with a wide range of physical and psychological disorders of an acute and chronic nature, including regular observation, administration of medications, nutrition, wound care, comfort care.

- Maternity post natal care.

- Child health nursing, either for health promotion in schools, health camps or sick childcare.

- Sexual health treatment compliance monitoring.

- Home care, district nursing care travelling across isolated communities in response to calls for assistance or in regular rounds to monitor the health and well-being of the community. 
- Setting up fever camps where outbreaks of infectious diseases were identified. This was generally with limited equipment and resources, nursing these people alone with the help of local communities. Also nurses worked in sanatoria to care for people requiring inpatient care.

- Working in tent hospitals and in existing buildings in Europe and North Africa providing immediate and acute care for injured soldiers and locals, returning on hospital boats with injured and rehabilitating soldiers.

\section{Achievements}

Nursing achievement in this period was obvious as evidenced by:

- Establishment of a health presence in most communities despite shortages of nurses to undertake backblock nursing.

- Successful containment of infectious disease outbreaks and limitation of deaths where this was possible.

- Satisfaction expressed by communities with the care and attention received to the point that communities preferred to pay for their half of a nurses salary rather than pay for a hospital distant to their farm; and perception of threat by medical staff where communities became more healthy.

- Improved knowledge by mothers of child health nutrition needs.

- Acceptable report of health status in comparison with other colonies.

\section{Responsiveness}

Nurses demonstrated responsiveness through the actions of the individual nurses working in the isolation of the back blocks, their ability to deal with all eventualities, different personalities, cultures, communities and conditions with minimal preparation and back-up. Not all were able to cope with these demands but these pioneer women responded to practice demands beyond their training and preparation. In going to Europe and Africa during war to provide care for the soldiers, nurses responded again to changed practice circumstances.

\section{How did nursing contribute to the health gain of New Zealand in this}

\section{period?}

The nurses demonstrated the diversity of what they could contribute in practising in the inpatient and community settings, undertaking more health promotion, disease containment and recovery treatment. They were prepared to travel in isolated communities in New Zealand as well as overseas with the Army Nursing Corps. They educated people; were able to offer wide-ranging advice and assistance; provided care; and saved lives. They assisted people to regain their health to resume their responsibilities. 
In the third and final case study in this chapter, it will be shown that communities had barely recovered from the influenza epidemic and war, when a global economic depression occurred that had a significant impact on the health and welfare of the New Zealand community. Then, barely had they recovered from this, World War Two was declared and New Zealand offered to provide support to Great Britain. Health services were barely coping with the demand for care and attention and were further stretched.

\section{Case Study 3. 1920 to 1945}

\section{Introduction}

This was a turbulent period for New Zealand. The population was recovering from the impact of the First World War. The returning soldiers had had difficulty settling back into New Zealand way of life after their experiences overseas. The Great Depression of 1930 introduced a difficult economically and politically and a desperate time for communities without alternative sources of income. The change of government in 1935 and the outbreak of the Second World War in 1939 placed considerable pressure on the community but also provided employment and opportunities.

\section{The Socio-political Context}

\section{$\underline{\text { Population }}$}

The census of 1920 estimated the population at 1,136,389. The Maori population was recorded at 73,289 . In 1930 the mean population was estimated to be $1,556,318$ and by 1940 the census reported the population at two million people with 1 in 3 people living in rural settings (Hyslop, 1989, 
p.30-31). Life expectancy for men was reported in 1935 as 65.46 years and for females 68.45 years.

Births had declined in $1918-1919$, the lowest since 1855 attributed to the impact of family disruption during the war and the influenza epidemic. In 1923 the average number of live births per married European woman was 2.4, in 1880 it had been 6.5 (Coney, 1993, p.71). Life expectancy in 1931 for European males was 65.04 years and 67.99 for females (Hyslop, 1989, p.48). The death rate was 10,808 or a rate of 9.51 standardised (it had increased to 14.66 in 1918). It was estimated that there were 70 deaths per 1000 illegitimate live births compared with 40/1000 legitimate births (Coney, 1993, p.76). Infant death rates were reported as $93.59 / 1000$ live births in 1934, down from 107.23 in 1925 . The greatest mortality in the 3-12 months of age group was from respiratory disease (AJHR, 1934, H-31, p.6).

Immigration increased during this period with British immigrants arriving each month, some on assisted passages. After the Second World War, Polish refugees were accepted into New Zealand as part of a resettlement programme and 800 Polish orphans were accepted into a camp at Pahiatua in the Wairarapa in 1945.

Political tensions: Following the First World War, the ANZAC Day Act 1920 designated April 25 as a public holiday in acknowledgement of the many people who had died in the war effort. The League of Nations confirmed New Zealand mandate over Western Samoa in 1920. The Ross 
Dependency in Antarctica was placed under New Zealand control in 1923 and administration of the Tokelau Islands was also assumed from 1925.

Maori continued to have a significant role in the political life of New Zealand. Ngata was appointed Native Minister in 1928. Princess Te Puea, of the Waikato 'iwi', worked hard to address the demoralised and fragmented ‘iwi' by re-establishing traditional patterns of communal social and economic life for the people of the Waikato. She based her initiatives on traditional culture and spiritual values and under traditional leadership assisted the Waikato Maori back to their identity and their pride, securing for them a position of strength to enable them to survive ion the pakeha world. Te Puea initiated the building of a model $\mathrm{Pa}$ at Turangawaewae Marae, Ngaruawahia, the Mahinarangi meeting house opening in 1929. This became a focal point for Maori values and activities including return to the land and economic self-reliance through farming. She sought to establish proper medical facilities to improve the health standards, but was unable to achieve her goal (Sargison, 1993, p.38). Other leaders such as Ratana called for ratification of the Treaty of Waitangi in 1922 . The Government awarded $£ 5,000$ annual land compensation to the Taranaki tribes as reparation for land confiscation.

The first Labour government won the election in 1935. The very popular Prime Minister, Michael Joseph Savage died in 1939 and was replaced by the Rt. Hon Peter Fraser, who was also the leader of the war cabinet. 
New Zealand declared war on Germany in 1939. Maori MPs asked that Maori be allowed to volunteer for a special unit as part of a Maori Battalion. Women were allowed to enter the armed forces although many women remained at home and worked in the "war effort" on farms and in factories. Special camps were established for war defaulters. Those remaining at "home" were concerned for their safety as a German mine sank the goldladen Niagra in 1940 and a German raider sank the Holmwood and Rangitane. A Japanese plane was also observed flying over Wellington and Auckland. Rationing was in place and there was control of industrial manpower to meet the needs of the army and of Britain. Conscription started in June for men aged 18 to 46 years.

Deaths during war were high per capita with 194,000 men conscripted into the armed forces of a total population of 1.5 million. New Zealand soldiers distinguished themselves in Greece, Crete, Bardia and Tobruk, with the Maori Battalion in particular acknowledged for their service. New Zealand declared war on Japan in 1942. US marines arrived in New Zealand in 1942 for shore leave and men from the United States and New Zealand forces brawled in Manners Street in Wellington.

Amendments were made to the National Service Emergency Regulations from 1942 and these declared that nurses were an essential industry (Kai Tiaki, 1943, July). In the Featherton camp where Japanese people had been interred, 49 Japanese prisoners of war were killed in a revolt in the camp. A Pacific Pact was signed with Australia in 1944 although the third division was disbanded as the Pacific role in the war ended. New Zealanders joined 
the J-Force with western forces in the war against Japan. New Zealand also led the small nations at the United Nations in 1945 and was nominated as a United Nations trustee for Western Samoa.

Social issues: Women continued to be active in working collectively to ensure that women and children were supported. The Family Allowance was introduced in 1926. The Country Womens Institute formed around social events and skill sharing, with an emphasis on craft and traditional country pastimes (Coney, 1993, p.298). The Girl Guide movement was officially introduced in New Zealand, the aim being to develop home craft and motherhood skills. The Womens Division of the New Zealand Farmers Union formed in 1925 in concern for the plight of back blocks women and worked to justify the expansion of rural women's roles and opportunities for education, medical care and domestic help. Self help store and co-operatives were established in response to the economic situation and growing skill development of women.

The Borstal system for young offenders was established in 1924. The 'environment versus heredity' debate was addressed by a Committee of Inquiry into Mental Defectives and Sexual Offenders and concluded that children should have healthier diets, more outdoor activities and proper rest. Censorship of films and books was favoured. The publication by Ettie Rout of a 'safe sex manual' for women, called Safe Marriage, which gave information about avoiding pregnancy and venereal disease, was banned in New Zealand although it sold well in Britain and Australia. This conservativism denied the reality of a pre-nuptial conception rate that was 
estimated at 33 percent, with illegitimate births at 5 percent of all births (Coney, 1993, p.178). In the 1930s, legislation was altered to allow divorce after three years of separation.

Appliances were imported and prices were prohibitive. Due to the economic difficulties, only 4,600 of 192,000 potential consumers had electric stoves in 1940. The economy started to improve after the Second World War and by 1956, over half of all homes had an electric stove and the same proportion had a washing machine and a refrigerator (Coney, 1993, p.214). Thirty-seven percent of provincial dwellings and nineteen percent of urban dwellings occupied by Europeans in the 1930s had a coal or wood range for cooking. Maori dwellings had fewer amenities. A census showed that 52 percent of Maori dwellings had no water laid on, 80 percent had no hot water and 64 percent had no electric light (Coney, 1993, p.212).

The Maori Arts and Crafts Act was passed and the Intertribal Carving School opened in Rotorua. The Maori Social and Economic Advancement Act was passed in 1945. Eighty-one percent of Maori lived in rural areas, and after the war this increased to eighty-eight percent living in hapu-based settlements (King, 1988).

Employment: There were 78.8 percent of males and 21.2 percent of females in the labour force in $1930,42.5$ percent of the total population or 63.5 percent of all 15 to 64 years old (Hyslop, 1989, p.30, 31). The number of women in domestic service reduced from 19,189 in 1901 to 17,955 by 1921 but this number surged despite the economic Depression to 29,262 in 1936 (Coney, 1993, p.225). The Government banned strikes and lockouts. 
The Great Depression of the early 1930s, with the high unemployment per capita, was a time of great strain on the rural and urban communities. Men were paid only three weeks out of four. As part of the Public Works No.5 Scheme for unemployment relief men were sent to tent settlements in the backblocks to work on certain projects such as road construction, railroad tracks and tunnel building. Women in employment were required to pay an unemployment levy of three pence for every pound from 1931, then in 1932 this increased to one shilling. If unemployed, women were not eligible for unemployment relief. Some unemployed women had no option but to sleep in doorways, which was denied by the Minister of Health. At the peak of the Depression in $1933,79,587$ or 17.8 percent of the male workforce over twenty years was unemployed. Women were not registered as unemployed although Hospital Boards and women's organisations attempted to identify the full scope of the problem. Women's unemployment committees were set up to administer the small amount of assistance.

Farm commodity prices collapsed. The Expenditure Adjustment Act of 1932 cut pensions, state salaries, rentals and interest; civil servant wages were cut by twenty percent and there were double and triple tax increases. There were unemployment riots in Auckland, Wellington and Dunedin. Food parcels were distributed. Unemployment peaked at 80,000 in 1934. Maori unemployed received $1 / 3$ cash and $2 / 3$ rations. Mortgage relief for farmers was increased in 1934 in an attempt to maintain production. The new Labour Government introduced a sustenance wage of ten shillings (half the rate paid to men) for unemployed single women. Unemployment decreased to 14,000 by 1938 . The Social Security Act was passed in 1938 as an attempt to ensure 
that all adults received free health care and had access to a reasonable standard of living. National Superannuation was announced and was to be financed from tax of one shilling in the pound.

The Police Force Act was amended in 1938 to allow the appointment of women but it was not until 1952 that uniformed policewomen were seen on the streets and 1972 when the first police prosecutor was promoted. (Coney, 1993, p.126).

Compulsory unionism was introduced in 1936 and the 40-hour week was formalised. The Labour Government introduced a graduated wage with automatic, age-based increases in 1936. Only 3.7 percent of married women were in paid employment in 1936. Twelve percent of Maori women and 22 percent of European women were participating in paid employment. While the two World Wars changed the nature of the female workforce and women's perception of what work they could do, women were expected to relinquish their work to returning soldiers (Coney, 1993, p.211).

\section{Health Issues}

Inpatient facilities: The national number of hospital beds increased from 9,234 (5.7 beds per 1000 population or 4.3 occupied beds per 1000 population) in the 1920 s to 13,870 in $1936 / 37$, to 16,613 by $1943 / 44$, that is from 8.4 to $10.2 / 1000$ population (Dow, 1995, p. 105). One person in sixteen aged 65 years and over had been treated in public hospitals (Hyslop, 1989, p.190) or $88 / 1000$ population treated in hospital (Hyslop, 1989, p.200). 
Maori resisted hospitalisation until the 1930s as they feared treatment in European institutions, which were frighteningly alien and far from the security of friends and family (Sargison, 1993, p. 39). In 1930, seventy-eight percent of European women and only seventeen percent of Maori women gave birth in hospital (Coney, 1993, p.61). There were six Karitane Hospitals around New Zealand training nurses in Plunket methods. A ward at the King George V Hospital in Rotorua was re-opened for children affected by poliomyelitis.

The population suffering form severe mental disorder in the eight public mental hospitals and one small private mental hospital in New Zealand at the end of 1943 numbered 8,122. This equated to a ratio of about 51.55 nonMaori patients per 10,000 of the general population and 19.59 Maori patients per 10,000 of Maori population. 1 person in every 202 of the population resided in a mental hospital. This number had dropped from 97.7 per 100,000 in 1916 (Beaglehole, 1958, p.28-30).

Health status data: A report written in 1918 reported a high accident rate: 538 deaths through accidents, excluding homicide, starvation and excessive cold. One hundred people were reported as having died from drowning. Railway accidents accounted for twenty-three deaths, twenty-two classified as serious injury and 732 minor injuries (New Zealand Journal of Health and Hospitals, 1919, p.30).

The overall death rate in 1920 was reported as $9.87 / 1000$ mean population, infant mortality 31.12/1000 live births, pre-maturity being recorded in almost 
half of the cases (AJHR, 1920, H-31). Maori mortality was reported at a rate of $16.95 / 1000$ population compared with 9.87 for European. From 1907 to 1938 European infant mortality fell from 88 deaths/1000 live births to 32 per 1000, the lowest infant mortality rate in the world (Coney, 1993, p.65; Hyslop, 1989, p.50). Maori infant mortality was reported at a rate of 102.26 /1000 live births compared with 30.12 in European children. Maternal mortality was reported at 2.22 per 1000 births for Maori compared with 2.65 for European.

The mean population was recorded in 1934 as 1,476,988. The birth rate was registered as 24,483 or $21.54 / 1000$ in 1919 . Deaths were registered as 10 , 808 as compared with 16,364 in 1918 and a yearly average of 9,730 in the period 1910-14. The standardised death rate was recorded at 9.12 per 1000 . The number of stillbirths had deceased with the rate recorded as 687 or 28.3/1000 live births compared with 865 or 32.2 in 1930. Heart disease major cause of death at 22.67 compared with 20.33 in 1930 (AJHR, 1930, H-31, p.13).

Health Service Organisation: Free health care was introduced in the changes to the Social Security Act 1938, to include free hospital services, free maternity benefits, health benefits, pharmaceuticals, x-rays and district nurse benefits. The provision of free services had a significant impact on the demand for inpatient beds and free maternity services. 
The Child Welfare Act of 1925 and the surveillance of children through the School Medical Service led to closer scrutiny of the health of foster children. The Mental Defectives Amendment Act was passed in 1928.

Epidemics, outbreaks and major health issues: Dental caries were identified as a major problem. A Dental Nurse Service was proposed in 1921 and the Dental Nurse training programme was established in 1922. The tooth extraction rate was reported at 400 per 1000 population at that time. At the outbreak of World War Two, sixty percent of recruits were reported as wearing dentures and 80 percent needed dental treatment.

A Health Board committee investigated the incidence of venereal disease as this had increased after World War One, from 156 people with syphilis in 1938 to 403 in 1941. Incidence of gonorrhoea peaked at 1,511 notifications (Kai Tiaki, 1941, September). The review concluded that parents were not educating their children about sex or birth control practices, the condom, cervical cap and diaphragm being identified as the most reliable methods of birth control.

In 1932 New Zealand held the unenviable record for having the second highest number of deaths from septic abortions out of eight Western countries. Between 1927 and 1935, 223 women died after septic abortions. A government Committee of Inquiry into the various 'Aspects of the Problem of Abortion in New Zealand' estimated that in the late 1930s at least 4,000 illegal abortions were being carried out each year (Coney, 1993, p.73). The Sex Hygiene and Birth Regulation Society was therefore established in 1936 
"to educate and enlighten the people of New Zealand on the need for birth control and sex education and to promote the provision of facilities for scientific contraception so that married people may space or limit their families in accordance with the requirements of health and economic circumstance". This society was renamed the New Zealand Family Planning Association in 1939. The medical profession vehemently opposed this organisation (Coney, 1993, p.72).

The incidence of infectious diseases during this time reduced for some disease and increased for others. Immunisation uptake was identified as poor in a study undertaken in 1936, which found that 83 to 85 percent of all school children were not vaccinated. This improved to 65 percent vaccinated by 1940 after a focused programme managed by public health nurses in local communities and schools. There was a scarlet fever epidemic in 1928 with 6,127 cases notified, reducing to 4,848 and 2,244 in 1929 and 1930 respectively. The rate increased again from 1,196 in 1943 to 7,612 in 1944 (AJHR, 1945, H-31). The cases of influenza were notified in 1919 at 8,277 compared with 9,303 in 1918 (AJHR, 1920, H-31, p.2). A diphtheria immunisation programme was initiated in South Auckland in 1933 and a reduction in diphtheria incidence was reported of 436 cases and 26 deaths as compared with 963 and 27 deaths in 1933 (AJHR, 1934, H-31, p.2). There were 45 deaths from whooping cough in 1927, which had a major impact on Maori infant mortality (Coney, 1993, p.65). Measles accounted for 46 deaths in 1938, compared with seventeen in 1933, the measles epidemic also having a major impact on Maori babies (Coney, 1993, p.64; AJHR, 1934, H-31, 
p.3). A poliomyelitis epidemic was reported in 1933 with 359 cases and 19 deaths.

Hydatids, brucelosis and undulant fever were identified health problems and legislation was passed to ensure clean milk supply. Hydatids incidence was reported in 1929 at $100-150$ per year, with a mortality rate of fifteen percent, the highest incidence noted in Canterbury and Otago (AJHR, 1934, H-31, p.4). Seventy-four deaths were reported in 1944 from enteric fever.

A Royal Commission was set up in 1923 after five maternal deaths were reported due to sepsis at the Kelvin Private Maternity Hospital in Auckland. There were 73 notifications of sepsis following childbirth despite almost universal use of sulphonamides; 59 deaths were from puerperal fever, seventeen from sepsis after childbirth and 42 from abortion (AJHR, 1945, H31, p.3).

Deaths from tuberculosis were reported in 1920 as 762 or $6.71 / 10000$ (Annual Report, 1920, H-31, p.3). A report published in 1935 on Tuberculosis in the Maori: East Coast of New Zealand reported the Maori death rate ten times higher compared with European. Sixty-nine percent of those identified with tuberculosis disease had not had previous medical treatment (Annual Report, 1939, H-31, p.7). Tuberculosis incidence peaked in 1945. The Department of Health cited tuberculosis as an occupational risk for nurses in 1933, with nurses contracting tuberculosis at a rate twelve times higher than the general population (Coney, 1993, p.103). While vaccination 
had been available since 1922, this option was not given to nurses. The first nurse was vaccinated voluntarily in 1948.

Treatment of war injuries was a priority in the period as many soldiers were injured physically and psychologically following internment in camps.

Childhood health assessments were undertaken and 64,323 examinations done: Dental caries were reported at 33 percent for European children and 49 percent for Maori children. Fillings were recorded in 6 percent of European children compared with nineteen percent of Maori children. Subnormal nutrition was reported at a rate of 6 percent for European children and 3 percent for Maori children. Ten percent of European children had goitre. Sixteen percent of Maori children were reported as having scabies.

Health promotion activities: Insulin use was introduced for the treatment of diabetes in 1933 (AJHR, 1934, H-31, p.19).

The Health Department launched a campaign for 'safe maternity' including aseptic techniques. Except in St Helens Hospitals, doctors attended most European births.

Child health assessments were undertaken in 1927 by Dr Gunn who reported that in the Otago area 25 percent of children were malnourished, 30 percent had breathing obstruction and 70 percent defective teeth. The 'Free milk in schools' scheme began in February 1937 in an attempt to provide nutritional supplementation for children. Not all children enjoyed this free supplement. By 1942, 83 percent or 235,701 pupils were receiving school milk (AJHR, 
1938, H-31). Free apples were also provided until 1948. This scheme was only discontinued in February 1967 (Hyslop, 1989, p.119).

The high incidence of goitre was regarded as a public-health problem and iodised salt containing potassium iodide 1:250,000 was introduced with a demonstrated reduction in incidence over the following five years from twenty-four percent to fourteen percent in 1929. Investigations extended to include a survey of water, milk and sanitary conditions in the Taranaki and Palmerston North areas. Health Camps established in the 1920s in Southland, South Canterbury, Auckland, Wellington, Christchurch, Dunedin, Raglan, Waikato, and Otaki were reported as offering significant benefit to children. Rotary Clubs helped by funding children (AJHR, 1940, H-31, p.35).

The Womens Health League was established as a widespread movement primarily of Maori women and was concerned with infant care, diet, improved sanitation, health education and care of the sick (Coney, 1993, p.89).

From 1943 to 1984, the "Radio Doctor" broadcasts were commenced by Dr Turbott. Topics included childhood ailments, abortion and birth control, immunisation and nutrition. A Good Nutrition booklet was promoted and was in high demand, with 40,000 printed.

Health workforce: The Army Nursing Service sent 640 nurses to the Middle East, Italian Campaign and Pacific Civil Defence in the Second World War. 


\section{Issues for nursing}

In 1917 nurses were appointed to work with the doctors in the School Medical service. The work of these nurses was described as "specialised work", with the nurses in the rural areas combining the school health work with their other work, seeing all children and arranging for them to see the doctors at regular intervals. The school nurses visited homes and advised parents where necessary as to the general care of the children. They arranged transport and treatment at a hospital if the parents were unable to take the children and could not afford private fees. The Child Welfare Act of 1925 highlighted general concern for the health and welfare of children.

The Sex Hygiene and Birth Regulation Society was established to promote the provision of scientific contraception and nurses were recruited to provide the education and assessment required.

The role of the District Nurse continued to be promoted with nineteen district nurses working among Maori by 1926 and twenty-five nurses employed in 1935. A new method of reporting Maori births and deaths was introduced, as previously this had been incomplete and actual census data is inaccurate. The district nurses were responsible for notifying death and possible cause.

District nurses in Whangarei and Gisborne introduced a health-teaching group concept whereby they educated the local community about certain issues. This increased the independence of the community so that they would only needed to call the nurse where practical on defined visiting days and could rely on a responsible person in the community to advise the nurse if an 
urgent case needed a personal visit. Demonstration material was limited and nurses often bought material and equipment themselves. The Provincial Federations of the Women's Institutes provided assistance with health education material focused on infant welfare for the older girls in primary and secondary schools, using a pamphlet in English and Maori. Some sessions were given by the senior women schoolteacher and the class was later examined by the district nurse (Kai Tiaki, 1934, May, p. 55-56).

Concern was expressed about the limited preparation of nurses to start work in the community and in public health nursing. This resulted in a proposal to increase the 'Public Health Nursing' content in the basic course (Lambie, 1956, p.15) and to consider additional post-registration nursing education. "Hitherto we have sent them forth to this work with no preparation whatsoever" (Kai Tiaki, 1934, July, p.113). While trained nurses were perceived as being employed to work with activities of caring for the sick, preventing disease and promoting health, other special branches of nursing were developing. Work was increasingly available in areas such as district nursing, infant welfare, general antenatal and maternal welfare, mental hygiene, industrial hygiene, school nursing, tuberculosis and venereal disease nursing. A more broad-based training programme was therefore recommended.

In rural nursing a nurse is needed to be a general nurse, midwife, health visitor, school nurse and district nurse, requiring a very wide range of knowledge combined with an engaging personality. Courtesy, common sense, sympathetic understanding and good sound judgement were as 
essential as professional qualifications (Kai Tiaki, 1937, December, p.329).

Post-registration nursing education was established in 1928 at the new postgraduate School for Nurses in Wellington that was a joint venture between the Department of Health and Victoria College. Nursing post registration education was encouraged.

Nurses were warned that there were several aspects of hospital work where New Zealand lagged behind and where if the profession did not prepare with the necessary qualifications it would be found that others had specialised and would take the work of nurses, such as, dieticians. A number of specialist roles were suggested such as 'nurse dietician' as

nurses must know how to feed patients, she knows how much a patient can eat, knows the home conditions of the patient in order to advise on buying of food, and instructing people about how to make up diets for diabetics and cardiac (Kai Tiaki, 1937, December, p.326).

The role of 'the Almoner for hospital social services, or social worker [as we know the role today] was also a developing role thought suitable for nurses (AJHR, 1935, H-31, p.56-57).

Despite increasing need for nurses to work in New Zealand hospitals and communities, exchanges of nurses to other countries also commenced in this period, with nurses travelling to Melbourne, Fiji and Shanghai. New Zealand nurses were also sent to Samoa, Niuie, Rarotonga, Aitutaki and Norfolk Island to advise on their health services. 
Preparing enough nurses to cope with the increasing demand for workers provided a challenge to local hospital boards and the Department of Health. Training Schools had been established in 28 hospitals. Through the 1920s there was a major recruitment initiative. During the 'Great Depression' of 1930, availability of positions had an impact on nursing recruitment and employment, with number of nurses either not paid or laid off, by 1935 employment was stabilised. The 1938 Social Security Act had a further major impact on the requirement for nurses as free hospital and district nurse services increased demand for health care and nurses. Staffing of sanatoria for treatment of people with tuberculosis was difficult due to the limited number of nurses and the isolation of the facilities away from towns.

With the onset of the Second World War, all 'manpower' was controlled in order to meet the needs of the communities at home and the need of the soldiers in Europe and the Pacific. Registered nurses were expected to volunteer to practise their profession unless special circumstances indicated otherwise, when exemption had to be obtained from a District Manpower Officer, acting on the recommendations of the Department of Health. Recruitment and retention continued because after the war concluded public hospitals were faced with a large increase in their occupied bed rate from Returned servicemen who still required treatment, and there were a large number, were accommodated in the public hospitals. Newer treatments, such as antibiotic drugs and blood transfusions, necessitated patients entering hospital for treatment. Early ambulation was introduced and this meant that the turnover of acutely-ill patients 
was much more rapid; and added to these factors was the free hospital service introduced under the Social Security Act, which encouraged patient admission and length of stay (Lambie, 1956, p.149).

The District Nursing Benefit was introduced in 1946 as a free service to provide bedside nursing in the home to people discharged early or those with chronic illnesses.

During this time period, the outstanding feature was the continuous demand for nurses to work in an increasingly diverse range of roles. The nursing resource was spread across the town and rural settings, in more specialist roles and settings, in war zones and in rehabilitation.

\section{Questions raised by the case study:}

How did nursing contribute to the health gain of New Zealand in this period of extraordinary 'ups and downs', high growth in demand for nurses?

Using the framework tool developed from the 'Contribution Model', the actions of nursing in response to need and expectation can be profiled. The work of nursing is structured in Table 8 as follows.

\section{Table 8. Contribution framework for the period 1920 - 1945}

\section{Expectations}

\section{Stakeholders}

During this period, more nursing was occurring in the increasing number of hospitals across the country and less nursing occurred in homes, except for the district nursing role in urban and rural settings.

Some nurses worked in backblock and community nursing with isolated Maori communities. Nurses worked in child health camps to improve nutrition and child health, in health education to prevent the spread of venereal disease. Nurses also undertook Army nursing during World War Two and rehabilitation nursing with returned soldiers. So what were the expectations? 
Expectations of nurses by patients (inpatient)

- Efficient management of the health services.

- Adequate numbers of qualified nurses and midwives and probationers to meet the diverse needs of work in the general acute, maternity, private hospitals and sanatoria. "Registered nurse numbers increased from 492 to 984 between 1936 to 1941 and student numbers 1491 to 2798". "Refresher courses with 1540 registered nurses volunteering to return"; "nursing engages 8364 women nationally and 400 overseas" (Kai Tiaki, 1941 November, p.1; Kai Tiaki, 1943, February, p.1; Kai Tiaki, 1942, February, p1; Kai Tiaki, 1943, March).

Expectations of nurses by patients (community-based)

- Nurses able to work anywhere: in schools, Plunket child health nursing, district nursing, dental nursing, outpatient clinics, tuberculosis and venereal disease, industrial nursing and Red Cross (Kai Tiaki, 1937 March, p.60).

- Able to make assessment of the full range of health problems, accidents, births and deaths that presented; to plan and direct care whether the medical practitioner was present or not.

- Able to make health assessments of communities and offer advice on environmental hygiene management, nutrition, health maintenance and disease prevention.

- Venereal disease assessment, treatment compliance monitoring and teaching (Kai Tiaki, 1937, March, p.78).

- Health promotion teaching (Kai Tiaki, 1943; AJHR, 1919, H-31, p.14).

- Nurses with comprehensive knowledge of infectious diseases and isolation management (AJHR, 1919, H-31, p.14).

- Nurses working in war zones: "362 nurses in Army Service" (AJHR, 1919, H-31, p.14; Kai Tiaki, 1942, November, p.1).

- Expectations of nurses by decision makers

- Adequate workforce supply to meet the demand for staff for hospitals, community work, the war effort "programmes offered in 28 schools" (AKHR, 1935, H-31, p.53).

- A full range of services would be available to achieve the promises of a free health service to meet the increasing demand for care.

- " "good standard of service" (AJHR, 1934, H-31, p.64).

- "Records of work carried out" (AJHR, 1935, H-31, p.56).

- "inspection of hospitals continues with full measure of co-operation" (AJHR, 1935, H-31, p.40).

Expectations of nurses by Medical Practitioners

- Intelligent and educated nurses able to implement the care prescribed and call for assistance as required. Probationers supervised adequately.

\section{Purpose of the nursing role}

To provide a full range of accessible health care services, advice and education for individuals and families in towns, isolated communities and overseas using available resources and working in co-operation with the others health team members. 


\section{Knowledge, skills and expertise required}

Nurses needed

- A wide clinical knowledge base to cope with the range of health presentations, for hospital and community care whether medical assistance was available or not.

- In-depth knowledge of infectious diseases, infection control, isolation techniques with continuous outbreaks of infectious diseases and the impact of tuberculosis.

- Knowledge and experience in the care management of people recovering from accidents or acute illness or dying.

- To be able to deliver babies and deal with childhood illness.

- To be able to cope with major lacerations and injuries without access to medical assistance.

- Ability to assess whether someone could remain at home or be transported with difficulty to a hospital some distance away and confidence to direct the transport process.

- Skills to cope with people with mental illness in the community and those who did not comply where intervention was required.

- Ability to teach and advise on self care, health promotion issues including immunisation.

- Confidence to address sexual health issues in assessment, reinforcing treatment and education for self care.

\section{Anticipated outcomes}

It was anticipated that the following outcomes would be achieved.

- Nurses would be present in situations to facilitate community access to health care in an environment of increasing access to free health care.

- Treatment, convalescence from ill health.

- Management of infectious disease outbreaks and minimisation of risk of spread, rehabilitating people once treated.

- Safe delivery of babies and establishment of breast-feeding and mother craft.

- Improved knowledge about nutrition and health practices.

- Improved child health through intervention in schools.

- Treatment and rehabilitation of soldiers from the effects of war injuries.

\section{Actions}

Nursing actions included:

- Care of patients in hospitals, in the community and in Army hospitals providing care for a full range of physical and psychological disorders of an acute and chronic nature, including regular observation, administration of medications, nutrition, wound care, comfort care.

- Provision of maternal and postnatal care.

- Child health nursing either with health promotion in schools, health camps or sick childcare.

- Sexual health treatment compliance monitoring.

- Home care, district nursing care travelling across isolated communities in response to calls for assistance or in regular rounds to monitor the health and well-being of the community. 
- Sanatoria care for people requiring inpatient care for treatment of Tuberculosis and convalescence.

- Working in tent hospitals in Europe and North Africa providing immediate and acute care for injured soldiers and locals, returning on hospital boats with injured and rehabilitating soldiers.

- Mental health nursing in inpatient settings.

\section{Achievements}

Nursing achievement in this period was obvious as evidenced by:

- Maintaining health presence in all settings required to provide service.

- Successful containment of infectious disease outbreaks.

- Satisfaction expressed by communities with the care and attention received.

- Reduction in child and maternal mortality through improved obstetric nursing practice.

- Reduction in incidence of venereal disease through effective treatment, awareness and education.

\section{Responsiveness}

Nurses demonstrated responsiveness through their willingness to undertake more and more to meet demand and expectations. They initiated recruitment for more nurses, planned nurse preparation programmes and exhorted non-practising nurses to return to work to ensure adequate coverage. Nurses were also prepared to travel overseas to war and to other countries to offer assistance and advice.

Did nursing contribute to the health gain of New Zealand in this period? From the literature it is clear that there are more similarities in nursing contribution through these time periods than differences. There were consistent expectations and achievements evident. The need for order, organisation and cost containment in hospitals and health systems was evident throughout. Nursing assumed a leadership role in achieving these expectations as situations changed. The standards of care and service were improved and this enhanced the trust relationship between nursing and the communities that were served. As community needs became more evident, nursing responded flexibly to meet the diverse needs, working in traditional roles as well as nontraditional, isolated places using their knowledge and skills in different ways. The differences in nursing contribution across these time periods are less 
obvious with a more transitional approach from traditional to more diverse, from narrow perspective on individual patients to broad community approach.

Nursing through these periods was increasingly diverse, undertaking whatever they could in response to identified needs. There was increasing demand for nurses to work in the hospitals. Nurses were required as community nurses; to work in new teams working with children and school health; to undertake industrial nursing and health promotion; to undertake sexual health nursing including health education, treatment monitoring, teaching about contraception; undertake maternity nursing as there was increased demand because of free hospital care, fewer private hospitals and increasing birth rate; nurse in sanatoria for people with tuberculosis; and undertake army nursing. The demand for more nurses further increased with nurses called to work overseas as part of the Army Corps. This left a limited number of experienced nurses able to supervise the increasing number of students.

\section{The contribution of nursing during these time periods in history}

From the literature, there is evidence that the contribution of nursing to the health of New Zealand during this period was significant. Nursing entered a health service that was in disarray. They proceeded to organise, manage, coordinate and deploy nurses wherever required. Nursing established systems and processes so that standards and expectations were clear. Nurses were trained in increasing numbers to cope with the increased demand. This ensured the continuance of an organised service delivery. Nursing also encouraged nurses to go anywhere and do anything that was required, often at personal cost and with limited preparation or resources. This flexibility and 
responsiveness spread the resource very thin at times but nurses made sure that they were present regardless, that they provided the care required, that healthy living and practices were promoted health and worked in areas of health restoration across a broad diversity of practice settings. While there are few measures that can provide evidence of economic contribution, the literature is relatively "rich" with description of the expectation, action, achievement and responsiveness. The descriptions in the literature profile the caring in the human health experience, expressing the humanness of nursing contribution in health (Litchfield, 1999).

Nursing exists to improve the health status of patients entrusted to his or her care and to improve the health care delivery system. If that is the raison d'être of nursing, then my assessment is that nursing achieved this in the time periods studied.

In the next chapter, the 'contribution model' is applied using examples of specific specialist roles and functions. This is a different approach, narrower in focus, yet it reflects the reality of contemporary practice that is more specialised, mirroring the increasing medicalisation of healthcare over the past thirty years. 


\section{Chapter Six}

\section{Nursing contribution in the present}

This chapter explores the contribution of nursing as a modern profession, a profession that continues to contribute as it did in past decades (Chapter 5) but has also become more specialised in roles and functions. Nursing in contemporary practice settings, that is practice as it has evolved over the past two decades, has retained many aspects of the core practices of the past but has altered in response to recent advances in technology and changes in service delivery expectations. The questions explored are: is nursing contribution as evident today as it was in past decades? Can the 'Contribution model' assist nurses articulate what they contribute? This chapter continues the discussion of contribution but focuses on the issues facing nursing in the early $21^{\text {st }}$ Century, considering the application of the 'contribution model' in the context of articulating scopes of speciality practice.

The application of the model continues the use of a case study method, although in this chapter the observed roles or practice settings are the focus rather than the general descriptions of practice across the health service as was the approach in the previous chapter. This approach is different from the previous chapter and has been used because practice occurs in more specialised settings than in the past and it is in these specialised settings that many nurses are having difficulty articulating their value and contribution. The application of the model to an individual role or scope of practice demonstrates another way in which the 'contribution model' can be shown to 
be flexible. By using a case study approach with information from literature and observation, the 'contribution model' provides an example of how the scope of practice can be used to describe the focus, functions and outcomes.

Nursing today is a product of past expectations, action and responses as well as connection and influence to current requirements and realities. Nursing is also influenced by a number of factors, such as: approaches in the preparation for entry to the profession; professional socialisation issues; personal and collective experiences; the traditional image of nursing in society; the political and economic climate of the day; and the impact of globalisation on the body of professional knowledge and demand for nurses. These influences on the current 'face of nursing' cannot be underestimated, as they influence the images that nurses have of the value of their own work, its contribution and its future. For many nurses, there is a sense of excitement and optimism about the future, but for others there is an underlying pessimism.

\section{Contemporary nursing and its contribution to health}

So what is the contribution of nursing in New Zealand at the beginning of the twenty-first century? This will be discussed using six case studies describing specific roles and scopes of practice rather than nursing practice across the whole health service. In this section the 'Contribution Model' will be applied to roles and functions in contemporary practice settings. This thesis was designed as a 'thought experiment', the information presented therefore as an exploration of ideas from literature and observation to create a description of how the role of function could be articulated. The methodology did not 
consider the use of focus groups as in action research or gathering of information as in grounded theory. Rather the information presents six case studies that are detailed from the use of literature and from personal observation as a nurse leader over some years of interacting with nurses working in these areas. The case study range used is by no means representative of the full range of practice options. These case studies have been selected as examples because they are different from the work of nurses in the past as outlined in the previous chapter or are nursing roles in these practice settings are under threat of change or replacement by other workers. Having talked with nurses in these practice roles or settings I believe I have a reasonable understanding of these roles, sufficient for this purpose.

Contemporary nurses practise in a range of settings, working in a wide range of direct care, specialist advisory, management, teaching and research roles. Nurses continue to undertake a 'problem identification and clinical problemsolving' role, anticipating and preventing potential problems. They undertake a 'helping' role including providing comfort measures and caring for patient's families. They participate in the 'teaching-coaching-mentoring' of others function with patients, families and colleagues. Nurses undertake an important 'diagnostic and monitoring' function to sustain physiologic functions, communicating the multiple clinical, ethical and practical perspectives essential to effective inter-disciplinary practice. Nurses manage rapidly changing situations and demonstrate effective skilled know-how to respond to a crisis, administering and monitoring therapeutic interventions and regimens. They monitor the quality of health care practices and take 
action to ensure the smooth organisation of the health care unit, demonstrating consistent work-role competencies and skilled clinical leadership (Benner,1984; Benner et al,1999). These competencies (Appendix 1) predominantly describe the role of the nurse in the personal health treatment and recovery functions and describe less well the health promotion and disease prevention areas of practice where there is slightly different emphasis. Nurses are in high demand nationally and internationally to provide safe and effective health services and are obviously doing something that is needed and required to meet the needs of communities around the world, despite moves to introduce generic health practitioners and practitioner assistants (National Health Service, 2000).

Over the past two decades there has been considerable specialisation in nursing both nationally and internationally, reflecting the change in medical specialisation and technological advancement. Nurses have increasingly adapted to work in speciality practice, such as intensive care, coronary care, plastic surgery, orthopaedics and cardiothoracic surgery. Nursing in the specialities, such as neonatal nursing and radiological nursing, has become increasingly complex as new technology and research have "pushed the boundaries' of what it is possible to do to improve health recovery. This increased complexity has necessitated further study and where previously nurses worked in a setting for many years to develop expertise rather than undertake post-registration study, this has changed. The number of speciality areas requiring staff with more specialised knowledge and skills has 
increased and post-registration education has now become essential to ensure safety and competence in practice in these specialised settings.

The increasingly complex work and use of technology has resulted in nurses being less willing to work in other speciality areas of the in-patient setting because of lack of confidence in the specialist practices required to care for certain patient groups. This specialisation has had an impact on generalist nursing too. In some areas specialist nurses have taken over work from the 'generalist' nurses and in other settings unqualified workers are doing tasks previously undertaken by qualified professionals. District nurses in some urban settings no longer do work such as maternal and child health nursing, as midwives have become 'lead maternity care givers' and paediatric nurse specialists have taken over care of children with acute and chronic health problems. Community and voluntary hospice settings as well as specialist home visiting nursing teams generally provide palliative care and do not utilise the generic district nurse. Some work previously done by nurses has been delegated to unqualified workers such as community support workers working with people with chronic mental illness. Operating Theatre nurses are increasingly challenged by the use of technicians. These changes are not universal but have altered the landscape of professional careers such that the community is confused about what is provided and by whom. These changes require that the profession is clear about what it is that is contributed and how the profession can work with other groups of providers to ensure that the community receives the care required and achieves health gain. 


\section{Settings and specialty practice areas}

In the previous chapter, the diversity of practice settings was listed to show the diversity, flexibility and responsiveness of the discipline of nursing to the demands of those time periods. A similar listing is worth considering as applying to modern nursing. The list, drawn from my personal experience, is long, diverse and complex, representing a profession that has adapted nursing knowledge and response to new technology to an ever-increasing range of services. In New Zealand, the following roles and scopes of practice are part of modern nursing opportunity for involvement.

Table 9. Settings and specialty areas where nurses work in contemporary practice

\section{Nurses work in In-patient Settings}

- Emergency Acute Assessment and Treatment.

- Ward or patient care units focused on the needs of specific diagnostic groups, for example: general physical health problems such as respiratory problems, surgical problems requiring surgical intervention, orthopaedic, neurosurgical, gynaecology, plastic surgery, respiratory, cardiology, aged care, rehabilitation, infectious diseases, oncology, renal, dermatology, gastroenterology, urology, haematology, vascular, trauma.

- Special high dependency units such as coronary care, intensive care, post-anaesthesia recovery areas, neurological intensive care, neonatal care.

- Radiology diagnostic and interventional units.

- Operating room nursing and post-anaesthesia recovery.

- Day procedure units such as cardiac investigations, pacemaker insertion, gastroscopy assessment.

- Mental health patient assessment, stabilisation, treatment, rehabilitation, crisis intervention, support.

- Psychiatric Liaison for assessment and care planning for patients and support of staff dealing with complex presentations.

- Paediatric nursing including neonatal nursing, acute assessment and stabilisation, surgical, medical, oncology problems, rehabilitation and respite. 
Nurses work in In-patient Settings, continued

- Long-term care of elderly and disabled.

- Infection control nursing.

- Outpatient clinics for initial assessment, pre-operative assessment, post procedure and ongoing follow-up, sexual health assessment and followup.

- Ambulatory care procedure units such as cardiac interventions, renal dialysis, surgical procedures, and blood transfusion

- Nurse educators of patients with specific health management problems including cardiac rehabilitation and where staff require development in order to provide safe and effective care.

- Nurse specialists offering care co-ordination, stabilisation and management of chronic health problems such as diabetes, thromboembolic disorders, chronic obstructive airway disease or patient education such as asthma management.

- Nurse specialists co-ordinating the processes of care for patients undergoing transplantation.

\section{Nurses work in Community-based care settings}

- Communicable diseases contact tracing.

- Mental health crisis team assessment and intervention.

- Mental health community support monitoring District nursing for posthospital care during convalescence, ongoing wound care including ulcer management, stoma management, supervision of administration of insulin and other medicines, administration of antibiotics, support of family members caring for people with chronic health problems.

- Public health nurses providing child health monitoring, assessment and support for children under five years old where families are at risk, assessment of school aged child health, vaccination campaigns, health education, child abuse investigation, family health actions including nutrition, budgeting.

- Paediatric specialist nurse roles in acute care and chronic health family support.

- Plunket nurses focused on early child health.

- School health nursing.

- Ear nurse specialists.

- Chronic mental health.

- Health promotion education and targeted health programmes.

- Occupational health nursing.

- Practice nursing attached to general practices.

- Independent practitioners.

- Private nursing of people in their homes.

- Hospice and palliative care for the terminally ill.

- Respite care for caregivers needing assistance and rest.

- Case management of people undergoing rehabilitation such as head injured people.

- Community emergency care clinics. 
Nurses work in Community-based care settings, continued

- Care of some people in their homes where previously they were admitted such as maternal mental health patients, certain acute mental health presentations.

- Supervision of care of people in hostels and sheltered accommodation.

- Telephone triage nursing.

- Community health clinics.

- Surgical clinics where procedures are done by surgeons such as cosmetic procedures such as liposuction, skin peeling.

- Prison nursing.

- Refugee support and integration.

- Community development activities.

Nurses work in Administration settings

- Professional leadership and advisory roles.

- Policy advice.

- Project work.

- Research and evaluation.

The range of opportunities where nursing is practised is diverse and has extended over the past decades. Some services have divided into public and private sectors, with increasing voluntary and community supported initiatives. This has occurred as: competition has increased with the introduction of privately owned emergency clinics; as public agencies no longer provide 'non-core' services; as specialised in-patient facilities have closed, such as the Mangere Hospital for people with intellectual disability and large mental health hospitals such as Kingseat and Carrington Hospitals in Auckland. Community agencies have developed to meet identified needs where other services have discontinued.

In most instances there is still a need for nurses to provide care and coordination with the wider health service system. That could change, however, with the expanded numbers of caregivers, mental health workers and patient care assistants. The range of settings where nursing is practised has created 
more options for nurses to work in narrower specialities as well in generalist care settings.

In 1993 the government organised health into a purchaser-provider model and sought health providers to establish and manage health services. The large public hospitals and health services and some private providers continued to be the main health service provider organisations. The health planners had anticipated more nurses would apply to manage contracts for the provision of new initiatives for care co-ordination and reference to the barriers was made to this in the Ministerial Taskforce on Nursing in 1998. Some contracts for service were awarded to nurses in rural communities such as in Wairarapa and in some urban speciality practices, such as Well Women's Nursing Services in Auckland. The reason that the number of nurses prepared to negotiate an independent provider contracts for service were lower than expected, was that many nurses had difficulty describing their purpose, what they could contribute, how they would organise themselves working with medical and allied health groups. For many nurses there was also less incentive to seek to establish independent practice because of the high demand by major public health employers for nurses with specialised expertise in existing provider organisations. Also many nurses need financial security and were unwilling to work under pressure to become an independent providers.

A number of nurses, however, have taken the initiative and currently manage practices to meet the health needs of communities, particularly in Maori health initiatives. As smaller hospitals have closed and rural communities 
have experienced difficulty retaining their general practitioner, a number of nurses have identified themselves as primary health providers practising comprehensive generalist nursing practice, referring directly to medical specialist services for more intensive assessment and advice.

So how could the 'Contribution Model' assist a nurse in contemporary practice to articulate their contribution to health outcomes? This will be answered by applying six case studies to describe the expectations of nurses working in these settings, what actions they take to meet the identified needs of the consumers and what achievements they can and do demonstrate. The six specialty areas are shown in Table 10 .

\section{Table 10. Practice settings where case studies arise}

- Acute care nursing in a surgical ward working with women undergoing surgery for breast cancer

- Operating Room or Theatre nursing

- Radiological nursing

- Nursing in an acute Mental Health in-patient and crisis team work, in

- Specialist nursing co-ordination role for people with chronic obstructive airways disease

- District Nursing

It is intended that these case studies will consider similar dimensions to the previous chapter including what is happening in health and how nursing is responding to the identified need. The achievements consider efficiency and effectiveness although the aspect of financial impact is not developed in this thesis. The impact of nursing involvement in reducing risk or improving efficiency by reducing the length of inpatient stay is highlighted. Each case study is a snapshot of practice. The purpose is not to create a definitive description of the practice reality but rather to demonstrate how the 
'Contribution Model' can be applied to help the nurse or group of nurses articulate their contribution.

\section{Case Study 4.}

Practice setting: Acute surgical nursing for breast cancer

\section{Introduction}

An increase in breast cancer incidence has been reported in many countries, with mortality generally increasing in western countries prior to 1990 although this is now levelling off in countries such as United Kingdom, United States, Norway and Sweden. The incidence of breast cancer is greatest in women 45 years of age and older. Since 1960 the numbers have shown higher incidence in women 65-74 years of age (Ursin, Bernstein \& Pike, 1994). In Australia, the age-standardised incidence was 62.6 per 100,000 woman years in the years between 1983 to 1987 . In New Zealand the incidence was 64.3 per 100,000 for the same period and in the United States of America 89.2 per 100,000. Between 1983-1987, mortality in Australia (22.1 deaths per 100,000 woman-years) was lower than in England and Wales (30.3 per 100,000) and New Zealand (26.4 per 100,000).

The incidence of breast cancer has increased in New Zealand over the past decade so that 13,522 women in New Zealand each year $(2.4 \%$ of total Disability Adjusted Life Years (DALYs)) can anticipate having a breast lump that will require investigation, monitoring and treatment (Ministry of Health, 2001, p.24). For women a breast lump will be a major event that has life threatening implications as well as an impact on self-image and confidence. 


\section{Socio-political context}

The prevalence of breast cancer in women has become a major political issue, as breast cancer is the single biggest cause of death among New Zealand women, aged 55 years and under. Women are advised to have a mammogram every two years after the age of fifty and between the age of forty and forty-nine on the recommendation of their general practitioner. The national mammogram screening programme is provided free for women aged fifty to sixty-four who have not been previously diagnosed with breast cancer in the previous five years. Overseas experience has shown that deaths from breast cancer can be reduced by up to thirty percent with regular screening. While there have been administrative and clinical reliability problems with the national cervical screening programme, the government is still committed to providing the national breast screening and referral process.

\section{Health issues}

\section{Health service organisation}

While there are a number of medical specialists and clinics offering private specialist service, which can offer nearly immediate assessment and intervention for women with breast lumps, general practitioners still refer women to the public health service for assessment and urgent intervention when required. This is because most people in New Zealand do not have private insurance. Most District Health Boards are required to offer or be able to offer prompt assessment of women referred and treatment within agreed timelines. Breast Care Services are attached to Surgical Services in most district general hospitals nationally and many services have a clinical 
nurse specialist employed to co-ordinate the referrals and the episode of care of women presenting for diagnosis and treatment.

The national Cancer Society provides voluntary support to women and their families where there is diagnosis of cancer. There are other Breast Care support groups in most centres and women may be referred or self-initiate access to care as appropriate.

\section{$\underline{\text { Health promotion }}$}

Considerable work was done to increase awareness amongst women of the need for regular self-assessment of their breasts for lumps and irregularities. Information about available services is also advertised in womens magazines and in primary health centres. Recent evidence suggests that there should be less emphasis on personal checking and more use of mammograms for women over the age of forty years. There are annual programmes for national awareness and a range of poster and pamphlet displays, available in all primary health centres encouraging women to seek screening.

\section{Issues for nursing}

While breast cancer is a women's health issue and is ideally managed by specialist nurses with primary interest in womens health nursing, the service is managed as part of the surgical services of most District Health Boards. There is generally a multi-disciplinary collaboration to ensure quick initial assessment and diagnosis of woman presenting for attention. The role of the specialist nurse ensures that the woman receives the most appropriate information by someone who has considerable knowledge of the trajectory of 
the disease and treatment process. The nurse is also able to co-ordinate the health team effectively to get the answers and support the woman and her partner requires. If the intervention includes surgical treatment, the nurse specialist supports the woman at the first specialist assessment and provides continuity of care as the central contact point, being present as much as possible. As the woman progresses from diagnostic assessment through surgery, convalescence to completion of the oncology treatment and discharge, guiding the surgical generalist nurses providing components of care with a clear clinical care plan, the nurse remains the constant.

So how might the 'Contribution Model' communicate the value that the speciality nurse provides in this situation. This is shown in Table 11.

Table 11. Contribution framework outlining the work of the breast care nurse specialist role.

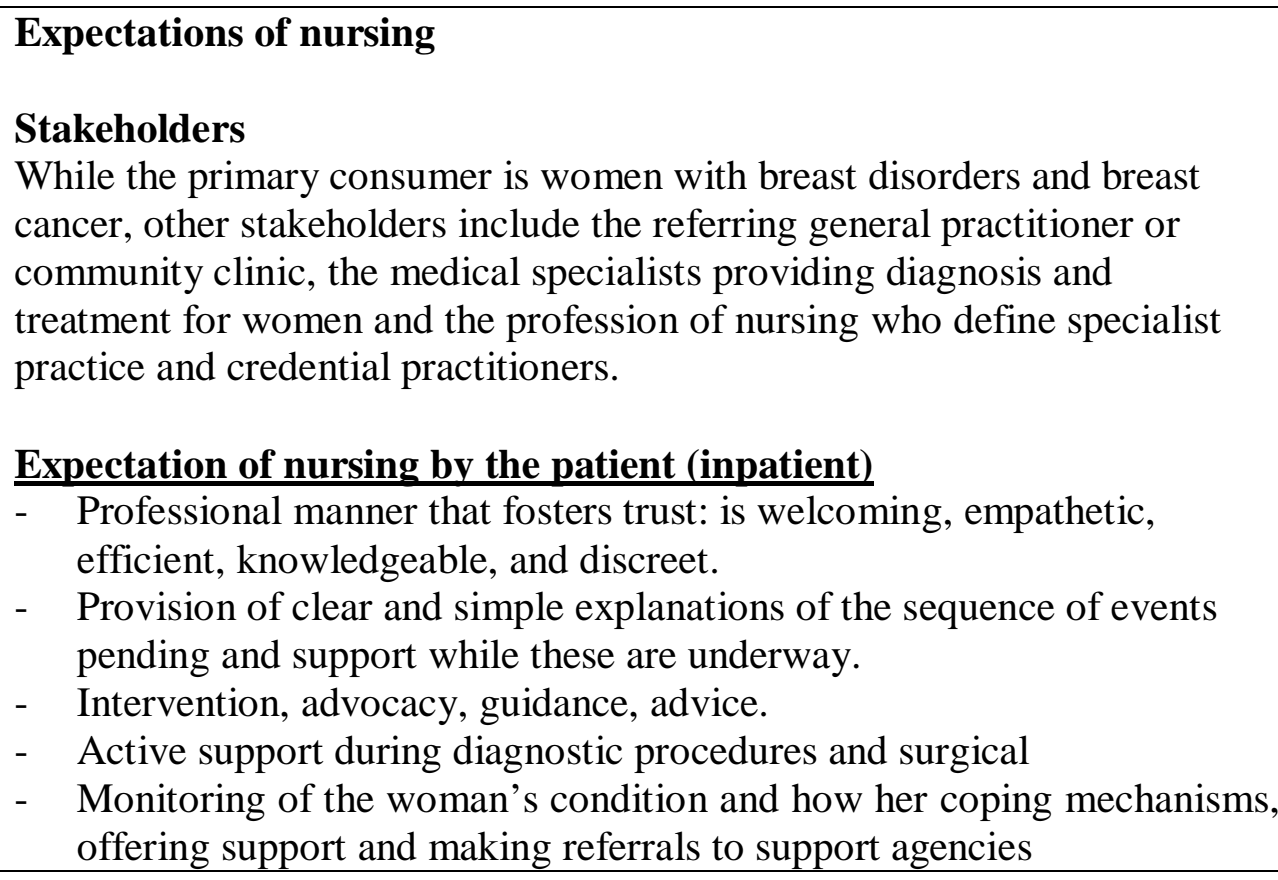

Expectation of nursing by the patient (inpatient)

- Professional manner that fosters trust: is welcoming, empathetic, efficient, knowledgeable, and discreet.

- Provision of clear and simple explanations of the sequence of events pending and support while these are underway.

- Intervention, advocacy, guidance, advice.

- Active support during diagnostic procedures and surgical

- Monitoring of the woman's condition and how her coping mechanisms, offering support and making referrals to support agencies 


\section{Expectations of nursing by the patients (inpatient)}

- Appropriate assessment and pain management is offered: assessment, advice and referral as necessary.

- Advance explanation and personal support when the woman is receiving the diagnosis and discussion about the prognosis and when viewing the impact of the surgical intervention as regards body-image impact.

- Preparation for self care, after care and treatment by careful explanation and support.

- Counselling as required and ability to manage expressions of grief.

- Assistance to get a prosthesis.

- Referral to community support agencies.

\section{Expectation of nursing by politicians}

- Appropriate targeting of women in the district with health awareness programmes.

- Reliable and efficient service for those referred with no delays, appropriate standards of care offered.

- Reporting of problems so that issues can be addressed before they become a risk.

- Cost effective service with best outcomes.

\section{Expectation of nursing by health planners \& administrators}

- Participation in regular and consistent community awareness programmes.

- Monitoring of referrals received and triage of urgent presentations to ensure timely to minimise waiting list delays.

- Efficiency and effectiveness to meet service specification as per contract.

- Treatment using appropriate technology and accepted care pathways.

- Appropriate referral for ongoing care.

\section{Expectation of nursing by medical practitioners}

- Specialist and skilled nursing triage of referrals and prompt alerting of medical staff for assessment and to address issues of concern.

- Support and co-ordination of the care requirements for women presenting for care.

- Women are supported and ready to be able to work with the medical staff to plan care appropriate to their needs. 


\section{Expectation of nursing by the nursing profession}

- Appropriately experienced and educated nurses only undertaking this work, with post graduate qualification with speciality practice.

- Practice evaluated with peer review and personal reflection.

- Complications minimised through early identification and initiation of appropriate treatment.

\section{Purpose of the nursing involvement}

To provide information and support to women facing a potentially lifethreatening diagnosis, preparing her for the diagnostic and treatment procedures, monitoring the woman's recovery from surgical intervention in preparation for self-care and after-care on discharge.

\section{Knowledge and skills required}

- Understanding of the patho-physiology of the disease

- Experience with the anticipated treatment plan and expected interventions

- Care co-ordination expertise and knowledge of appropriate community support services

- Skill to manage grief reactions in relation to loss of body image

- Expertise to facilitate co-operation and motivation

- Expertise with wound healing strategies, especially where there are lymph collections

- Knowledge of community resources and support services

- Pain management approaches and knowledge of resource teams

- Techniques for post-procedure recovery of arm function

Anticipated outcomes of nursing intervention

- Patient confidence and participation in preparing for the procedure

- Uncomplicated recovery from the procedure

- Appropriate wound healing

- Resumption of self-care and normal function

- Appropriate referral to community support resources

- Maintenance of a positive body image

\section{Nursing Actions / Interventions}

The diagnostic and monitoring function

Administering and monitoring therapeutic interventions and regimens The helping role.

Physiological (basic)

Activity and exercise management

- Exercise promotion: review and encourage ambulation and arm range of movements on side where surgery occurred

- Teaching: prescribed regarding activity/exercise.

Immobility management

Limb positioning: review post-operatively and until swelling reduced, combat the hazards of immobility. 
Nursing Actions / Interventions, continued

Physical comfort promotion

- Environmental management: encourage comfort measures while in bed

- Pain management: Interpret kinds of pain and refer to the Acute Pain Team for appropriate strategies for pain management and control

- Provide comfort through touch and encourage family support

Self care facilitation

- Self-care assistance: anticipate patient care needs. Encourage grooming to maintain body image. Maximise the patient's participation and control in her own recovery

- Tube care: teach about self- care of wound and drains after discharge

- Prosthesis care: refer for fitting of prosthesis and practice in use of prosthesis to maintain body image

Physiological (complex)

Drug management

- Patient-controlled analgesia: monitor usage and coach patient to use appropriately

- Chemotherapy management: prepare for treatment by Oncology team and introduce to Oncology nurse specialist for ongoing/post-discharge care

Peri-operative care

- Teaching pre-operatively: what to expect and how family can provide assistance

- Surgical preparation: advise of pre-procedure preparation including physical and psychological preparation

Recovery post-procedure: assist general surgical nursing team as necessary to prepare the environment for post-operative care. Monitor at intervals the care of the patient after the procedure the general surgical nurses to seek to minimise the chance of complications. This includes pain management, action to minimise the risk of impaired physical mobility related to lymphedema, nerve or muscle damage and pain, risk for injury related to compromised lymph, motor and sensory function in affected arm.

$\underline{\text { Skin and wound management }}$

- Incision site care: review at intervals and detect and document changes in a patient's condition.

- Skin surveillance; review at intervals and detect, document and initiate treatment for lymphedema.

Wound care close drainage: advise and make appropriate referral for wound care including removal of drains, drainage of seroma, monitoring of inflammation and healing. Create a wound management strategy that fosters healing, comfort and appropriate drainage. 
Nursing Actions / Interventions, continued

\section{Behavioural}

Coping assistance

- Body image/self esteem enhancement: Support the patient coping with acceptance of the changed body shape, especially when first visually sighting the surgical site

- Coping enhancement: Provision of emotional support, counselling and guidance as the woman adjusts to events, observing for and supporting the grief related to loss of breast and change in appearance

- Presencing: being with the woman, being available without being intrusive. Comforting through connection and relationship

- Eliciting and understanding the patients' interpretation of their illness

- Grief work facilitation

- Making culturally avoided aspects of the illness approachable and understood: ensure the woman receives tissue for burial. Deal with sexual issues and concern about partner reaction.

- Referral to support group

\section{The teaching-coaching function}

Patient education

- Teaching: individual: Provision of information and answers to questions. Preparation of the patient for self care of the wound on discharge, teaching treatment techniques and how and when to access assistance, exercised, breast prosthesis, signs and symptoms of complications, hand and arm precautions

- Capturing a patient's readiness to learn

Psychological comfort promotion

- Creating a climate for and establishing a commitment to healing

- Creating comfort measures and preserving personhood in the face of pain and emotional stress

- Anxiety reduction strategies

- Calming technique

- Assisting the patient integrate the implications of the illness and recovery into their lifestyle

\section{The effective management of rapidly changing situations}

\section{Safety}

Risk management

- Infection control: monitor team practices. Monitoring for signs of

- Infection.

- Complication management: Safety assessment prior to the procedure. Monitoring for restricted arm movement. 
Nursing Actions / Interventions, continued

\section{Family}

Lifespan care

Family support: Provide emotional and informational support to patients' families. Ensure that the family can be with the patient. Providing the family with information and support. Encourage family involvement in care giving activities

Monitoring and ensuring the quality of health care practices Organisational and work-role competencies

Health system

Health system management

- Examination assistance and physician support

- Co-ordinate the planning of the weekly clinics, liaise with the clinic clerk

Information management

- Documentation

- Multidisciplinary care conference: present at the meeting

- Referral: Preparation for discharge including referral for community support services and communication with the general practitioner as primary provider for follow-up care

- Telephone consultation: provide regular contract

Main taxonomy headings: McCloskey \& Bulechek (1996); Benner (1984); Benner, Hooper-Kyriakidis \& Stannard (1999).

\section{Achievements}

\section{Efficiency measured by}

- Number of women supported through similar procedures; achievement of contract targets.

- Percentage achievement of post-procedure standards of nursing care for care of women post breast surgery.

- Number (percentage) discharged within anticipated recovery plan timeline or earlier.

\section{Effectiveness measured by}

- Functional status: Potential complications anticipated and managed proactively.

- Symptom control: Seroma drainage managed with minimal disruption to lifestyle. Pain managed so that limb movement encouraged.

- Patient satisfaction: Woman stating satisfaction at being able to communicate her anxieties and need for information and support. Participation in health care decisions. 


\section{Effectiveness, continued}

- Client behaviours: Woman able to resume self care on discharge and utilise support resources appropriately. Grief resolution.

- Patient satisfaction: Expressed satisfaction with care provided. Selfesteem maintained.

\section{Responsiveness}

\section{The nurse specialist demonstrates}

- Expertise to identify potential complications and make referral for assistance.

- Expertise beyond technical skill to respond to the emotional adjustment needs of the woman and her partner.

In this case study the role of the nurse is specialised and focused narrowly on the episode of care of the woman experiencing a crisis in relation to breast cancer. The nurse undertakes a range of functions to provide support expected of the delivery system, the human face of caring. The nurse also provides the information required so that the woman can participate in decision making for her health. The nurses provide assistance with preparation and care during the procedure and facilitate recovery in resuming self-care and adjustment to altered body image. The contribution of the nurse is personal as well as technical, a co-partner in the experience, the advocate, monitor, and a coach. Nursing contribution is the technical expert but is equally involved with personal support in order that the woman achieves recovery, health gain and participation.

In this next case study the role of nursing is involved primarily in people support, co-ordinating monitoring function. The medical specialists and technicians focus on the technical accuracy of procedures to minimise risk and to achieve the most accurate diagnosis. The nurse manages the patient and their personal and safety needs, as well as co-ordinates the environment 
so that the specialists and technicians can provide efficient and effective response.

\section{Case Study 5.}

\section{Practice setting: Radiology nursing}

\section{Introduction}

Over the past decade the role of the nurse working in Radiology has altered from examination assistant and physician support to co-ordinator of the acute care team, the environment and monitor of the physiological care and support of patients undergoing increasingly complex procedures.

\section{Socio-political context}

Technology has advanced over the past twenty-five years such that procedures that were previously undertaken as surgical interventions under general anaesthetic are now done as day procedures or minimally invasive procedures, reducing the length of stay because of less physiological impact. While the cost of technology and specialist staff is high from an administrative sense, the procedures can reduce the number of diagnostic tests and more rapidly identify and deal with life threatening health problems. Health problems that were previously undiagnosed or identified too late can now be confirmed and appropriate treatment interventions initiated. There is cost benefit in these developments as treatment can be more appropriate and waiting lists for surgical intervention minimised. As technology has become more accessible, professional and community demand for these procedures has increased and this has put pressure on New Zealand health planners and administrators to make allocations for new technology and rationing decisions. 


\section{Health issues}

Inpatient facilities

There is increasing demand for less invasive procedures that minimise the risk of infection and restore the patient to self-care as quickly as possible. Facilities and equipment are increasingly developed in public and private centres to facilitate these ambulatory care procedures, using day procedure centres. Some patients still require inpatient admission for a few days, but this is less necessary with more effective pre-procedure assessment and preparation. These facilities are cost effective because of the high throughput and flexible hours of service.

Health promotion

The emphasis of ambulatory care is on self-care and partnership between the provider of service and the consumer. There is considerable emphasis on preprocedure explanation of what will happen, and ensuring that there is carer support systems available pre- and post- procedure, to ensure appropriate care on discharge. The consumer is preferably in the healthiest state prior to the procedure as possible so that they as less likely to require admission.

\section{Health workforce}

The medical, nursing and technical staffs working in these radiological environments are increasingly specialised and are difficult to recruit and retain. There is a need to have a stable supply of specialised staff, from a safety perspective and a service sustainability perspective. A significant amount of the work undertaken now and in the future will supersede the work of surgeons. For example, elective abdominal aortic aneurysm repair is now 
increasingly corrected through radiological procedures, angioplasty and stent implant. This change in practice could have implications for specialist physician and surgeon recruitment in the future, as new technology could mean that some of the current treatment options may be replaced by new less invasive treatments.

\section{Issues for nursing}

The range of procedures being done using new technology will require considerable upskilling and retraining on an on-going basis. While nurses are essential to this process, because of the complexity and acuity of the patients presenting for procedures, this is not always appreciated by the specialist and technical staff who view their own technical work as more important. Nursing's contribution is not so much the technical expertise but the coordination of services and people, patient support and monitoring and coordination of response in emergencies.

How might the 'Contribution Model' communicate the value that the nurse provides in this situation. This is shown in Table 12.

Table 12. Contribution framework outlining the work of the radiology nurse role.

\section{Expectations of nursing}

\section{Stakeholders}

The consumers in the first instance are the patients referred for the procedures. This may be any person of any age group requiring diagnosis or treatment that can be best achieved through radiological intervention and who can tolerate the procedure in their acute or stable health state. Consumers also include the radiologist specialists, radiographers and sonographers and other health team members with whom the nurses liaise. 


\section{Expectations of nursing by patients (in-patient)}

- Professional manner that instils trust, is welcoming, sympathetic, confident

- Clear information and co-operative planning for self care discharge

- Assessment of risk and appropriate alerting

- Assistance in preparation for the procedure including sedation, medication

- $\quad$ Support during the procedure if anxious

- Monitoring of physiological response and initiation of emergency procedures if necessary

- Post procedure recovery

- Preparation for discharge

Expectations of nursing by medical specialists and technicians

- Environmental management of the unit, including procurement, imprest management and scheduling

- Efficient preparation of equipment and the patient for procedures

- Monitoring of standards and providing a back-up system

- Efficient movement of patient through the procedures to ensure effective use of time

- Efficient management of emergencies requiring minimal supervision

- Co-ordinating and managing multiple instantaneous therapies

Expectations of nursing by other team members

- Professional manner in dealing with referrals and requests

- Clear instructions and scheduling

- Professional and prompt reception and handover of the patient

- Efficient co-ordination of the team in the event of an emergency, orchestrating their actions

- Detection and documentation of significant changes in a patient's condition.

Expectations of nursing by health planners \& administrators

- Skilled scheduling to minimise waiting lists

- Efficient ordering of equipment

- Minimisation of risk through anticipation and planning

- Preventing hazards in a technological environment by performing practical assessments, engaging in safety work and using equipment and interpreting its performance.

\section{Purpose of the nursing role}

Manage the environment, set-up of equipment and resources to receive and support the patient and health team before, during and after the procedures to achieve a safe and effective outcome. 


\section{Knowledge and skills required}

- Knowledge of the range of procedures, their uses for different needs, the patho-physiology and potential complications and equipment used

- Knowledge of the potential complications and how to manage crisis situations

- Knowledge of acute health presentations and co-morbidities and the appropriate nursing care as many patients are very unwell when presenting for a procedure

- Aseptic technique, sterile draping and theatre techniques

- Intensive assessment skills to monitor changes in a patient's condition and alert the medical and allied health team to emergency response

- The place of the procedure in the diagnostic process in order to provide appropriate education and support to the patient

- Post-procedure recovery and monitoring

\section{Anticipated outcomes}

- All the equipment and resources are ready for the procedure including additional resources that might be needed anticipating potential problems

- Problems are anticipated through appropriate assessment and experience and the incidence minimised through appropriate action

- The patient is comfortable and supported during the procedure

- The procedure is completed promptly to minimise the risk to the patient

- The patient is not put at risk of infection because of staff use of appropriate aseptic technique during the procedure

\section{Actions/Interventions}

The diagnostic and monitoring function

Administering and monitoring therapeutic interventions and regimens

The helping role

\section{Physiological: Basic}

Immobility management: Positioning of the patient on the procedure table for safety and comfort, to prevent risk of pressure on peripheries. Coordinating the transfer of the patient from the table to the bed or trolley at the end of the procedure to minimise the potential for harm to patient and staff. Combating the hazards of immobility.

Neurologic management:

Neurologic monitoring: Peripheral sensation management. Intracranial pressure monitoring. Cerebral oedema management. Detection and documentation of significant change in a patient's condition.

\section{Physical comfort: promotion}

Pain management: limiting the impact of painful procedures

\section{Self-care facilitation}

Self-care assistance: dressing before and after the procedure 


\section{Actions/Interventions, continued}

Providing emotional and informational support to the patent and family

\section{Physiological: complex}

- Drug management: conscious sedation. Radio-opaque dye. Ensuring that the patient has the appropriate medications in preparation for the procedure.

- Starting and maintaining intravenous therapy: with minimal risk and complications, administering medications accurately and safely

\section{Peri-operative care:}

- Positioning: intraoperative: 'Surgical' assistance. Preparation of the aseptic environment for the procedure. Assisting the Radiologist with the procedure. Administering radio-opaque dye

\section{Skin/wound management:}

Insertion site care post procedure

\section{Tissue perfusion management:}

Bleeding reduction. Embolus precautions. Intravenous insertion.

\section{Teaching-coaching function}

\section{Behavioural}

Patient education: teaching preoperatively. Orientation of the patient to the processes and environment. Education of post-procedure care requirements. Coping assistance:

Support of the family/support person who may be anxious and require information and or teaching.

\section{The effective management of rapidly changing situations Safety}

Risk management: Problems are anticipated based on reported problems and assessment of the condition of the patient that might alter the course of events. Preparation of the environment appropriate to the needs of the patient and planned intervention. Assessment of the patient on arrival including potential risks. Review of documentation and preparation for the procedure, including information given and the need for more explanation in order to be fully informed. Monitoring the patient throughout the procedure and reporting on any changes. Setting up the environment for the management of a crisis. Preventing hazards in a technological environment.

\section{Organisational and work-role competencies}

Crisis Management: Initiating emergency procedures if required and coordinating the actions of the team members. Undertaking full assessment of the patient and monitoring patient response post-procedure until transfer or discharge. Sequencing and managing the logistics of rapid multiple therapies in response to a crisis. Skilled performance in life threatening emergencies. Rapid grasp of a problem and initiation of contingencies. 
Monitoring and ensuring the quality of health care practices

Organisational and work-role competencies

Health system

Management: Procedure requirements are planned based on referral letter and schedule. Allocation of the appropriately skilled nurse to scrub and assist. Anticipating the needs of the radiologist in the set-up for the procedure and using the runner to acquire equipment during the procedure. Education of the ward or unit staff on the procedures, rationale for requirements for preparation and monitoring.

Information management: Handing the patient over to the ward or unit staff for ongoing monitoring and treatment. Documentation.

Main taxonomy headings: McCloskey \& Bulechek (1996); Benner (1984); Benner, Hooper-Kyriakidis \& Stannard (1999).

\section{Achievement}

\section{Efficiency as measured by}

- Number of procedures undertaken which requires nursing support, increasing in number and complexity

- Timely organisation of the department to cope with planned and unexpected requirements

\section{Achievement}

- Readiness for scheduled cases and timely preparation for unexpected cases

- Cost effective procurement and imprest management

Effectiveness as measured by

- Patient Satisfaction: Patient satisfaction with reception and support during the procedure

- Safety/Risk Control: Management of potential emergencies and complications to minimise risk to patients. Minimal risk of pressure areas or nerve damage through careful positioning

- Safety: Availability of information resources for ward or unit staff preparing patients for procedures

\section{Responsiveness}

- Adaptability to changing procedures and new technology

- Ability to cope with a wide range of acute and elective presentations, unstable clinical situations and people experiencing high anxiety

- Readiness for emergencies and ability to co-ordinate the health team

In this case study the contribution of the nurse is predominantly in the coordination of the unit, in set-up of the environment, monitoring of risk, provision of care of the patient. The contribution is significant in ensuring the 
efficiency of service delivery and making the experience as pleasant as possible for the patient.

In this next case study, the essential role of nursing in the acute adult mental health setting is considered. Nurses in this setting work to ensure safety and dignity for all as well as maintain a therapeutic environment within which a patient may regain equilibrium as far as possible in anticipation of eventual self-care.

\section{Case Study 6.}

\section{Practice setting: In-patient adult mental health service}

\section{Introduction}

Services for people with mental illness have undergone considerable change in the past decade as the large inpatient hospitals have been closed and more services have been offered to support community living. Inpatient units are very busy and full, available to provide acute assessment, treatment and management and preparation for discharge once the consumer is considered stable. These units do not have adequate space or capacity for long-term care or intermittent respite.

\section{Socio-political Context}

New Zealand has followed the international trends in closing the larger inpatient facilities and placing increasing emphasis on community-based care. Assumptions have been made about the requirements for inpatient beds and the resourcing of community services to meet demand, such as community crisis and continuing care teams. The services are nationally under 
considerable pressure as there is greater demand than resources exist to respond. This places considerable pressure on the health team and support services. Depression, anxiety disorders and dementia occur fifth, seventh and eleventh of the top twenty causes of disability adjusted life years (DALY) lost, being respectively 3.6, 3.2 and 2.6 percent of total DALYs (Ministry of Health, 2001).

While mental illness exists in each community, society appears to prefer to ignore the issues until such time as there is a crisis in the community when someone harms another person or group, then there is considerable community and political reaction. While there have been 'injections' of targeted funding to increase the range and quantity of services offered, there continues to be major under funding for mental health services using a population-based funding model or according to the Mental Health "Blueprint", developed by the Commissioner in 1999 that was intended to provide a strategy for targeting resources and improved access.

\section{Health issues}

It is estimated that at any one time approximately twelve percent of people require mental health services, and that one in four of the population will need some form of mental health service in their lifetime. Health services in New Zealand are resourced to deal with the 'top three percent' of acute need, with $\$ 284.7$ million spent on mental health services or $6.8 \%$ of the total health expenditure (Ministry of Health, 1999). This means that existing resources are inadequate to deal with current acute demand. 
Recruitment and retention of health staff in this speciality is difficult and existing vacancies are difficult to fill. This is an international problem and is reaching crisis proportions. Recruitment from other countries compounds national problems, as the "recruitment packages" that are offered by other countries are bigger than those that New Zealand can offer.

\section{Issues for nursing}

Nursing works in an interdisciplinary manner in mental health under strong medical staff direction of care. Nurses work with little infrastructural support as medical resources are limited and nurses are required to make a number of difficult decisions based on available knowledge. There is high use of health care assistant staff who work with limited supervision and do not easily take direction from registered nurses. While there is an 'entry to speciality practice' programme available for new graduate nurses wanting to enter this clinical speciality, mental health is not as popular an area of nursing as other settings. There is limited post registration education available, which makes up-skilling and development difficult.

How might the 'Contribution Model' communicate the value that the nurse specialist provides in this situation? The special characteristics are drawn out using the framework and knowledge of the work of nurses in this work setting. This is shown in Table 13. 
Table 13. Contribution framework outlining the work of the nurse working in the inpatient adult mental health service.

\section{Expectations of nursing}

$\underline{\text { Stakeholders }}$

The patients or consumers are in the first instance the main stakeholders and these include people referred, either new or previously known, with acute mental health problems that warrant comprehensive assessment, intervention and treatment in order to re-establish confidence and competence in self care before discharge. Other stakeholders include families, medical staff, allied health team members and health planners and administrators.

Expectations of nursing by allied health staff

- Clarity about the care plan and patient progress according to the plan

- Inclusion in assessment and planning

- Assistance in planning activities to assist the recovery of the patient and provide meaningful occupation

- Completion of the plan as documented where possible to sustain therapy

Expectations of nursing by patients

- Professional manner that instils trust, is welcoming, sympathetic, confident, discreet

- Clear information about the sequence of events to be followed in assessment and stabilisation

- Listening and counselling where support is needed

- Maintenance of a safe environment so that they are not harmed or harm themselves

- A calm and respectful environment within which they may make sense of what is happening for them

- Monitoring of response to treatment and initiation of procedures if necessary

- Preparation for discharge, re-entry to the community.

Expectations of nursing by families

- Professional manner that instils trust, is welcoming, empathetic, confident, discreet

- Listening and counselling about coping strategies where support is needed

- Planning for a safe environment to ensure that the family members are not harmed and the client is also safe from harming themselves

- Inclusion in discharge planning where possible 


\section{Expectations of nursing, continued}

Expectations of nursing by medical staff

- Maintenance of a therapeutic environment that minimises risk and enhances recovery

- Skilled, experienced nurses able to assess and initiate appropriate interventions, monitor progress with treatment plan, contributing to multidisciplinary planning

- Management of emergency situations until medical staff are available

- Confident practitioner able to offer advice and suggestions for patient management

Expectations of nursing by health planners and administrators

- Cost effective mix of staff to manage the clinical and supervision needs of the patients

- Appropriate management of the care plan in order to achieve the outcomes as planned for prompt discharge

- Minimised emergencies situations necessitating additional resources being used or patient delay in achieving goals

- Reduced staff injury from emergencies

- Minimised public relations problems

- Movement of patients in order to deal with demand

\section{Purpose of the nursing involvement}

To manage a safe and therapeutic environment within which patients can be assessed and receive treatment to reduce their confusion and anxiety in order to re-establish self care.

\section{Knowledge and skills required}

- Knowledge of physical and mental health patho-physiology which might manifest in illness episodes

- Knowledge of mental health presentations and how to provide support and protection of the person until their mental health is restored

- Assessment of the needs of the patient in initial triage and consistently thereafter

- Ability to defuse and contain incidents of potential violence to protect the safety of the person, other patients and staff

- Administration of medication and monitoring of patient response or side effects to the medication

- Encouragement of the patient to learn new ways of coping and integration back into society through individual and group programmes

- Expertise in re-orientation and motivation of the patients

- Ability to assist families make sense of what is happening and learn new ways to deal with mental health problems

- Refer patients for social and cultural support to enhance their safety and integration on discharge

- Co-ordination of community support services relevant to the needs of the patient 


\section{Anticipated outcomes}

- The patient will be safe and protected until able to resume self care

- The patient will receive the treatment required to manage the symptoms and presentation

- The patient will have increased capability for self-care as a consequence of the learning and integration with community and support services.

\section{Actions/Interventions}

The diagnostic and monitoring function

Administering and monitoring therapeutic interventions and regimens

\section{The helping role}

Physiological (basic)

Assessment of possible mental health problem including affective disorders, anxiety and adjustment disorders, bipolar disorder, obsessivecompulsive disorder, paranoid disorders, personality disorders, schizophrenic disorders, somatoform disorders, substance abuse

\section{Disorders}

Activity and exercise management: energy management. Immobility management when in restraint.

Nutrition support: nutritional monitoring. eating disorders management. Dietary advice with certain medications

Self care facilitation: self-care assistance

\section{Physiological (complex)}

Drug management: medication administration and teaching about prescribed medications. Administration of medications when unable to manage independently. Monitoring of patient's response to treatment and reaction to the environment, to counselling and administration of medications until stable and discharged.

\section{Teaching-coaching function}

\section{Behavioural}

- Behavioural therapy: activity therapy. Behaviour management (selfharm, over activity / inattention, social skills, limit setting, impulse control training), mutual goal setting, self-responsibility facilitation, substance use prevention/withdrawal. Management of programmes so that there is opportunity for learning, rest, exercise and social interaction.

- Cognitive therapy: anger control assistance, cognitive restructuring, cognitive stimulation, learning facilitation, reality orientation, reminiscence therapy. 
Actions/Interventions, continued

- Communication enhancement: active listening, complex relationship building, socialisation enhancement.

- Coping assistance: counselling. Crisis intervention. Mood management. Presence. Security enhancement. Self-awareness enhancement. Selfesteem enhancement. Support group and system enhancement. Therapy group discussion with patients to help them make sense of what is happening to them and learn to recognise symptoms in order to seek assistance in the future

\section{Patient education: Teaching prescribed medication}

Psychological comfort: anxiety reduction. Calming techniques. Distraction. Simple relaxation therapy. Organisation of the environment to achieve a calm, managed routine which provides order and support for the patients.

\section{The effective management of rapidly changing situations}

Safety

- Crisis management: crisis intervention. First aid and resuscitation if required. Suicide prevention. Triage. Management of potential and actual violent situations to minimise harm and restore order. Sequencing and managing the logistics of a rapid multiple response to a crisis. Organising the team and orchestrating their actions during a crisis. Taking necessary medical action to manage a crisis when a physician is absent.

- Risk management: Abuse protection. Delirium management. Dementia management. Elopement/AWOL precautions. Engaging in safety work through environmental management: safety and violence prevention. Fall prevention. Fire-setting precautions. Hallucination management. Seclusion. Seizure management. Suicide prevention. Monitoring of the patients to ensure safety and anticipate problems which may increase anxiety, providing early warning where significant changes are observed in a patient's condition.

\section{Family}

Lifespan care: caregiver support. Family integrity promotion. Family therapy. Work with families to help them learn strategies to help their family member and survive as a family unit

\section{Monitoring and ensuring the quality of health care practices}

Organisational and work-role competencies

Health system

- Health system mediation: admission care. Discharge planning. Patient rights protection. Sustenance support. Assessment of the person on admission. Liaison with other teams to prepare for discharge support. Minimizing health care system failures in a destabilised work environment. Building and preserving collaborative relationships. 
- Health system management: examination assistance. Staff supervision. Team building to develop a community of attentiveness, skill and collaboration.

- Information management: documentation. Health care information exchange. Health policy monitoring. Incident reporting. Multidisciplinary care conference. Referral. Shift report. Telephone consultation. Presentation of information to assist with care planning. Coaching others in interpreting, forecasting and responding to patient transitions.

Main taxonomy headings: McCloskey \& Bulechek (1996); Benner (1984); Benner, Hooper-Kyriakidis \& Stannard (1999).

\section{Achievements}

Efficiency as measured by

- Number of people stabilised to resume self-care in the community within the anticipated recovery plan.

- Organisation of the systems and processes to ensure that resources are available to defuse and contain situations which could put people at risk.

Effectiveness as measured by

- Safety: Number of people at risk prevented from self-harm until able to make sense of their symptoms.

- Technical aspects of care: Compliance with treatment plan

- Caring. Continuity of care. Teaching and counselling. Satisfaction with the support, encouragement and motivation provided to assist individual stabilisation. Provision for patient rights.

\section{Responsiveness}

- Nurses cope with a wide range of mental health presentations and more patients than beds and staff have available.

- Ability to co-ordinate a wide range of patients, families and community agencies to achieve a coherent support plan.

In this case study the contribution of nursing is the skilled experience that allows for anticipation of risk and promotion of a therapeutic environment, where new behaviours can be learned and where the patient can regain selfcomposure and confidence. The contribution is in reinforcement of the care plan in order that the anticipated outcomes can be achieved. 
In this next case study the role of the nurse is about care co-ordination and health promotion, despite living with a chronic illness. The role of the nurse in this setting is that of coach, reflector, co-partner to the patient and their caregivers, as living with a chronic illness has a significant toll on the caregiver.

\section{Case Study 7.}

Practice setting: Co-ordination of care for patient with chronic obstructive airway disease.

\section{Introduction}

Chronic health problems are a challenge to manage for both primary and secondary services. Not only is the quality of life of the person impacted by the chronic health problem, but their ability to sustain productive employment and social involvement is also impaired. The family and support system of the person is often strained. There are frequent hospitalisations for treatment and stabilisation.

\section{The socio-political context}

A range of models of care have evolved for the management of people with chronic health problems as it is acknowledged that there is an impact on the morale of more than the individual affected and cost benefit in reducing the need for admission to hospital. Case management models have evolved and there is evidence that the outcomes are improved where there is co-ordinated consistent care management. Nurses are often the care co-ordinators as there is more than a respiratory component to the lifestyle changes required for effective self-management. 


\section{Health issues}

Chronic obstructive airway disease (COAD) is a disease state characterised by the presence of airflow obstruction due to chronic bronchitis or emphysema. The obstruction is generally progressive and irreversible. Admissions for inpatient management of acute exacerbations can reduce the patient's ability to undertake normal employment and social functioning. Average length of stay can be 7.45 days with variable care where care co-ordination is not present to develop a clear plan of care. The disease process requires careful management to: reduce symptoms; improve exercise tolerance and reduce the need for admission with acute episodes; and optimise the level of physical and psychosocial functioning by assisting them adapt their lifestyle for health promotion and illness prevention to ensure a satisfactory quality of life. Care co-ordination can achieve a significant reduction in length of stay particularly over "winter load" period, a reduction in the number of admissions over "winter load" periods and significant improvement in quality of life scores.

\section{Issues for nursing}

Nursing is ideally placed to assume the case management role for patients with chronic health problems. These roles required an experienced practitioner, able to work in a co-operative practice model in true partnership with the patient and their family to achieve the empowerment required. The nurse also needs to have particular skills to provide counselling, to motivate and empower the person to assume self-care. 
How might the 'Contribution Model' communicate the value that the nurse specialist provides in working with this people with this chronic health problem? This is shown in Table 14.

Table 14. Contribution framework outlining the work of the nurse working with people with Chronic Obstructive Airway Disease (COAD).

\section{Expectations of nursing}

\section{Stakeholders}

While the patient is the primary stakeholder, this includes people with chronic airway obstruction who experience acute episodes of illness necessitating hospitalisation for stabilisation and have serious restriction of their life style as the disease progresses. This is a terminal illness. Other stakeholders include the family or support people, general practitioner, physician specialist and allied health team.

Expectations of nursing by patients

- Professional manner that fosters trust, is welcoming, empathic, optimistic, pragmatic, calm

- Listening ability to assist with realistic care planning

- Accessible person to discuss issues

- Consistent person to monitor progress and exacerbations

Expectations of nursing by family support

- As for patient

- Able to set limits and be up front in addressing issues

- Able to access assistance and support services for respite

- Able to assist with grief counselling and palliative care as appropriate

Expectations of nursing by general practitioners

- Expert collaboration in planning an appropriate care plan. Collegiality

- Shared care

- Visiting patient in hospital and facilitating discharge

- Realistic evaluation of prognosis and advice regarding palliation

Expectations of nursing by physician specialists

- Consistent professional who moves across the continuum of care boundaries able to provide full perspective of care needs

- Consultation regarding need for admission or planning for home based support

- Assistance with stabilisation of exacerbation

- Discharge planning for early discharge

- Monitoring of progress and communication of deterioration 
Expectations of nursing, continued

Expectations of nursing by allied health team

- Clarity about care plan and where allied health team can add value

- Consistent report on progress

\section{Purpose of the nursing role}

To work with the patient, their family and general practitioner to develop strategies for maintaining quality of life and health, reducing symptoms and the need for in-patient care and reducing length of stay where inpatient care is necessary

\section{Knowledge and skills required}

- Knowledge of the disease process and patho-physiology, treatment options and prognosis

- Advanced physical and psychological assessment skills

- Ability to motivate and celebrate achievement of goals

- Research skills to gather information to measure improved indicators and quality of life

- Care co-ordination across the community-inpatient interfaces for shared care with general practitioner

- Pharmacology knowledge for best use of medications to manage symptoms and maintain health

- Palliative care knowledge for symptom management

Anticipated outcomes

- $\quad$ Reduced need for admission

- Improved quality of life

- Reduced length of stay

- Reduced mortality

\section{Actions/Interventions}

The diagnostic and monitoring function

Administering and monitoring therapeutic interventions and regimens

\section{The helping role}

\section{Physiological (basic)}

- Activity and exercise management: energy management. Exercise therapy: ambulation, stretching. Physical assessment and review of past health history in order to understand current health status and previous acute episodes which required active intervention. Review of hospitalisation patterns to identify causative factors

- Nutrition support: nutrition management and monitoring.

- Physical comfort: promotion. Environmental management. Progressive muscle relation. 
Physiological (complex)

Self care facilitation: Activities of daily living. Sleep enhancement. Shared development of a care plan to maximise health, identify symptoms and initiate appropriate intervention to prevent deterioration including prevention of infection, action when recognising signs and symptoms of complications.

Drug management: teaching about prescribed medication.

Respiratory Management:

Chest physiotherapy. Cough enhancement. Oxygen therapy. Respiratory monitoring.

\section{Teaching-coaching function}

Behavioural

Behavioural therapy: maximising the patient's participation and control of their life. Activity therapy. Mutual goal setting and patient contracting. Self-modification assistance. Self-responsibility facilitation. Smoking cessation assistance.

Cognitive therapy: eliciting and understanding the patient's interpretation of their illness and assisting them integrate the implications of their illness into their lifestyle. Anger control assistance. Cognitive restructuring.

Psychological comfort: promotion: Anxiety reduction. Biofeedback. Calming technique. Simple relaxation therapy. Comforting through connection and relationship

Communication enhancement: active listening. Complex relationship building. Socialisation enhancement.

Coping assistance: Guiding patients through emotional and developmental change. Coping enhancement. Counselling. Crisis intervention planning. Grief work facilitation. Mood management. Self-esteem enhancement. Spiritual support enhancement. Work to develop strategies to reduce anxiety related to breathlessness and fear of suffocation, sleep pattern disturbance. Grief counselling and adjustment to changes in condition and prognosis.

Patient education: health education. Teaching prescribed activity/exercise, medication. Health education and goal setting relating to effective airway clearance, maintenance of nutritional status, improving exercise tolerance. Monitoring of progress and impact of illness episodes, liaising with general practitioners and specialists as required. Reviewing of the condition on admission and initiating an intervention plan to gain control of symptoms and effect early discharge using effective support structure.

Discharge plan initiated immediately to minimise delay in return to home. Treatments to maintain the person at home as long as possible. 
Actions/Interventions, continued...

Safety

Crisis management: Emergency care. Crisis intervention.

Risk management: Allergy management. Infection control. Vital signs monitoring.

\section{Family}

Lifespan care:

Caregiver support. Family integrity promotion. Normalisation promotion.

Respite care. Work to develop strategies to improve social support structure related to impaired verbal communication related to dyspnoea and powerlessness related to lifestyle restrictions. Reinforcement and motivation of achievements.

Being available without being intrusive. Comforting through rituals and routines. Working through decision points and transitions. Planning and implementing attentive palliative care

\section{Monitoring and ensuring the quality of health care practices}

Organisational and work-role competencies

Health system

Health system mediation: Decision making support. Discharge planning. Health system guidance.

Health system management: critical path development. Supply management.

Information management: documentation. Multidisciplinary care conference. Order transcription. Referral. Telephone consultation.

Main taxonomy headings: McCloskey \& Bulechek (1996); Benner (1984); Benner, Hooper-Kyriakidis \& Stannard (1999).

\section{Achievements}

Efficiency as measured by

- Reduced need for admissions due to health maintenance programme, early recognition of symptoms, self initiative for treatment, lifestyle and symptom management

- Reduced length of stay through prompt initiation of treatment and planning for anticipated discharge

- Reduced cost through reduction in bed days and improved use of resources

Effectiveness as measured by

- Improved quality of life due to longer periods of health maintenance and positive self-management. Symptom control. Functional status.

- Expressed satisfaction with partnership and achievements.

- Improved consistency of patient care and education.

- Availability and access to care.

Responsiveness

Skill in engaging with the motivation level of the individual and family Willingness to be available at all hours of the day to support the decision of the patient 
In this case study the contribution of the nurse is to empower the patient to self-manage their condition through education, integrated goal setting and motivation. The contribution is also to assist the patient regain control where exacerbation occurred and plan for discharge as soon as possible rather than relay on variable inpatient management. The contribution of nursing has financial benefit but is primarily aimed at health gain, despite a chronic prognosis.

In this next case study the role of the nurse is primarily that of the coordinating force that ensures increased efficiency and improved risk management. While there is technical skill in assisting with instrumentation, the role of the nurse as monitor of risk and safe practice is significant.

\section{Case study 8.}

\section{Practice setting: Operating theatre nursing.}

\section{Introduction}

Nurses have had a significant role in the Operating Room surgical team since the beginning of the last century, when surgical intervention was less well developed than currently. The role of the nurse included: cleaning of the operation rooms; scrubbing and sterilisation of the instruments; administering light anaesthetic under the direction of the surgeon if another medical practitioner was not present; assisting the surgeon with the procedure; recovering the patient from the anaesthetic; and cleaning in preparation for the next case. During World War One and Two and then in the wars in Korea and Vietnam, skilled operating room nurses were in high demand and it is in this capacity that the role of the nurse was pre-eminent (Kendall \& Corbett, 1990). In all hospitals nationally and internationally, nurses have been the managers 
of the operating room setting, managing the scheduling, deploying of staff, procuring equipment and monitoring standards of care and practice. While many of these same actions are part of contemporary practice, nurses perceive themselves under threat from decisions to employ anaesthetic and surgical technicians. While this threat many be real, nursing in New Zealand has been unable to articulate clearly what it is that is important about the nursing role in the operating room.

\section{The socio-political context}

The decision to introduce technicians has arisen over the past two decades, under pressure from the College of Anaesthesists who have documented the need for a technician in their standards of practice (Australia New Zealand College of Anaesthesists, 1998). These standards have stated that anaesthetists require a technician able to check the specialist equipment and medications prior to a case, to assist the specialist anaesthetise the patient and monitor the machines and patient stability under the supervision of the anaesthetist. Anaesthetists interestingly do not routinely use technician support in private sector hospitals but require this support in the public sector hospitals. Internationally, although not in New Zealand, nurses have undertaken advanced practice roles such as Nurse Anaesthetists. This role requires post-graduate education at an advanced level and the same indemnity as medical anaesthetists in this high-risk scope of practice. New Zealand has not progressed this role because nursing has not been able to prescribe and the recently acquired limited prescribing would still not allow for practice in this speciality. Medical staff are adamantly opposed to nurses undertaking this role and have actively refused to consider nurse 
specialisation in the past decade. It may become possible in time, but this is unlikely due to the power of the medical Professional Colleges.

As more surgeons return from work in the United States of America, they have suggested introduction of surgical technicians, suggesting that a technician as well as a nurse can undertake the activity of scrubbing and passing instruments to assist the surgeon. In the United States of America, the physician assistant (PA) profession has been established since the late 1960s and have worked as surgical first assistants and provided pre- and postoperative care to patients as part of surgical teams. Nearly 20 percent of the estimated 38,000 PAs clinically practising work in surgical specialities or sub specialities. The American College of Surgeons and the Society of Thoracic Surgeons recognise PAs as qualified first assistants (American College of Surgeons, 1999).

With the current workforce shortage with fewer nurses seeking employment in operating room settings, pragmatic decisions are being made in some hospitals. It is, however, worth reflecting on the reason for medical staff preference for unlicensed support workers. It could be postulated that there is a professional-political tussle underway, as nursing has always directed the 'how' of nursing in clinical settings and is not prepared to do what they are told. Some nurses have challenged medical staff with reported examples in Timaru in 1899 of nurses keeping a record of the doctor's attendance hours and in Auckland in 1891 where an inquiry into surgical standards was prompted by the complaint of a probationer. The Commission of Inquiry into the 1891 complaint was to be more critical of the nurses' disloyalty than the 
surgeon's error (Belgrave, 1989, p.22). Local medical practitioners have not always welcomed nursing input: “the nurse's work (perceived as) keeping the people in too good health for their (doctors) practices" (Belgrave, 1989).

While 'conspiracy theory' is irrelevant in this debate, there is evidence of medical staff preference for workers who have undertaken 'training' directed by medical staff and preference for 'physician assistants' who will work under the direction of the medical practitioner without challenging them. Medical staff also correctly perceive that nurses are busy with their own professional work in meeting the needs of the patient and are less likely to stop their own work to actively assist the medical staff. Contemporary nurses increasingly perceive a collegial relationship with the medical staff, each with their own professional knowledge, role and function to assist the patient achieve the desired outcome. This perception of peer relationships is not universally shared.

Nurses have developed confidence to challenge medical staff using evidencebased practice and will question directions and will interrupt medical staff practice and team decisions if there is any question of risk to patient or community safety. An example is the 'whistle blowing' incident in 1995 where a nurse, working in Mental Health, took the decision of planned discharge of a psychiatric patient to a Member of Parliament in order to prevent perceived risk to the community (Privacy Commissioner, 1996). Nurses are increasingly aware of their personal, professional and legal accountability as registered health professionals. Nurses are directly challenged about their actions, decisions and omissions and are questioned 
about the provision of a reasonable standard of care by the Health and Disability Commissioner, Privacy Commissioner, Accident Compensation Commission and patients. No longer is it acceptable for medical staff to direct care stating that the nurse must follow as they (the doctor) will answer for the actions of the nurse.

Recent Commissions of Inquiry in New Zealand, in the past two decades, have challenged nurses for failing to question team practise and for accepting direction from medical staff when they had a duty of care to debate professionally and undertake an advocacy role. Nurses have, in two recent Ministerial inquiries, been challenged about their failure or refusal 'to confront openly the issues arising' in medical practice. In two reports following inquiries, nurses have been identified as not speaking out to protect patient safety. The inquiries questioned the medical treatment of cervical cancer at National Womens Hospital (Cartwright, 1988) and also the provision of chest physiotherapy treatment provided to pre-term babies at National Womens Hospital (Cull, Weston \& Adams, 1999). A professional ethicist considering the first case stated that by

not acknowledging or dealing with the constraining effect that doctors and hospital administrators have on nurses' ability to act morally, the commissioner (Cartwright) in effect preserved the status quo, rather than challenged it... Unless the problem of institutional constraints on nurses is confronted, and unless the issues of hegemononic power relations within health care institutions is addressed, the danger of 'unfortunate experiments' occurring again, not to mention moral 
malpractice and negligence, will continue to prevail"' (Johnstone, 1989, p.16).

It is acknowledged by nurses the profession has a responsibility to advocate, especially where safety is paramount. While employers also have a responsibility to support nurses in working in this way, in reality advocacy is generally perceived as stimulating conflict.

The changing medico-legal climate has influenced the relationship between the medical and nursing professions. Medical staff have sought assistants to assist and monitor equipment and processes, often a support worker who will focus on their professional needs. This preference does not necessarily intentionally threaten the place of nursing in this setting, but acts to meet their own professional needs for support. The technician does not often question or challenge medical practice.

The introduction of alternative health workers is not a new phenomenon. After the Second World War the roles of dietician, physiotherapist, social worker and occupational therapist were introduced to meet the specific needs of patients. There were not enough nurses interested in undertaking these roles and they did not have the narrow specialised education required for these roles, although nurses had undertaken aspects of all of these functions and still do in many situations. While tensions have arisen with these groups and the role of the nurse, co-existence and mutual respect has been achieved over time. With the increasing shortage of nurses internationally it is possible that the role of the nurse in the operating room may alter significantly as time 
progresses. It may be that acceptance of technicians may be forced as the clinical functions are defined as narrow and there will be limited nurses to undertake this 'scrub' function. It may be that the role of nursing might evolve into the co-ordination and direction of care.

New Zealand managers, as part of the globalisation of health initiatives, have also perceived that the technician is a less costly alternative to the nurse. This has proven to be an economic mirage, as the salary of the technician is similar to that of the nurse and because of the shortage of highly trained technicians, they command a high price. In a redesign evaluation at Auckland Hospital in 1997 (unpublished), it was found that the technician role was in fact less economical because the technician had a limited scope of practice and were not able or willing to undertake the wide range of activities required of an operating room complex. Technicians did not have the comprehensive knowledge and experience of a nurse and were also less likely to identify general risk in the environment. They were also less effective in patient interactions. The fact that this project was not published is unfortunate because it does not allow for evidence to prove the nurses case.

Nurses do have a role in the Operating Room but it may not be limited to the technical task of instrumentation but involve the wider co-ordination and risk monitoring function across the Operating Room complex. Another role for nursing may be the 'first assistant role' as some nurses use their knowledge of patho-physiology and surgical procedures to anticipate the need of the surgeon. Tasks do not define the practice of nursing. The Operating Room 
nurses need to move beyond the instrumentation task to define their practice. The practice of nursing is the integration of a wide knowledge base and experience that ensures that nursing is able to assist the patient experience a safe and appropriate outcome.

\section{Health issues}

While there are increasing treatment developments using minimally invasive procedures, there is growing preference for non-invasive procedures, as society understands the risk of infection and the benefits of quicker recovery using laparoscopy and laser technology. The New Zealand health system has over time rationed the types of surgical procedures that can be undertaken in public hospitals and there has been a corresponding increase in 'waiting lists' as demand has increased for hospital treatment that does not require additional payment. No longer are relatively minor elective procedures done in the public system. This change has occurred because of government decisions to reduce waiting lists for major procedures where infrastructure of the public hospital is available and to boost private hospital and practitioners as an option. The cost of increasingly complex procedures, which have become possible through scientific and technological development, has meant that for cost and clinical safety reasons these procedures need to be done in resourced secondary and tertiary settings. There was an attempt in the 1980 s to discourage reliance on the publicly funded services and encourage use of private insurance. The increasing number of surgeons in private practice supported this. Society has accepted user-pays and private health care in part, although they still rely on the public health service for acute and complex health care needs. 
Concern about infection rates and the increasing production of 'single use' items has increased the cost of surgical procedures and there is strong reliance on infection control surveillance to ensure safe outcomes. There is also strong pressure on surgical teams to make best use of available theatre time to schedule and complete cases efficiently. 'Production planning' has become a major function of the theatre nurse manager role. Credentialing of surgeons has also become a major feature of professional practice as increasingly complex new technologies arise and patients require evidence that the surgeon has been trained in the procedure or use of equipment to achieve the best outcome (Ministry of Health, 2000).

\section{Issues for nursing}

While the profession perceives threat for an ongoing role in this context there are clearly a range of activities that nursing is best skilled to undertake. Nurses in this setting have not always maintained their post-registration learning to enhance the scope of practice. Instead they have relied on 'on the job' learning and experience. This has resulted in a perception of vulnerability and focus on task as the unique differentiation rather than the value of the comprehensive clinical, co-ordination and teamwork skills. Post-registration education is essential to prepare nurses in this setting for the broader role that is inevitable.

How might the 'Contribution Model' communicate the value that the nurse provides in this situation. This will be shown in Table 15 . 
Table 15. Contribution framework outlining the work of the nurse in the operating theatre.

\section{Expectations of nursing \\ Stakeholder}

While the patient as recipient of intervention is the primary stakeholder, other stakeholders include the surgeons, health planners and administrators and technicians. The patient as consumer is any person requiring surgical intervention to diagnose, stabilise or treat a condition that may be life threatening or elective, may be done under general anaesthetic or the patient may be awake and aware of what is happening.

Expectations of nursing by patients

- a professional manner - that rapidly forms a connection as the patient enters an unknown and 'alien' environment. Welcoming, empathetic

- consistency of person until the patient is anaesthetised so that the patient feels safe

- to have their risks identified so that problems can be anticipated

- to have systems and processes work smoothly providing a perception of safety and efficiency

- that they will have someone monitor that the right procedure is being undertaken.

Expectations of the nursing by medical specialists

- that the systems and processes will function to the highest efficiency, that resources will be available, that everything will be ready on their arrival

- that they will have a consistent, experienced and appropriate team of people ready to assist them, including the 'scrub' nurse, runner and other specialist staff

Expectations of the nursing by medical specialists

- calm and professional nurses who have current knowledge and skill in their practice and are ready as new procedures and developments evolve

- all risks to be identified and appropriate prompt action to prevent harm and deal with emergencies

- appropriate audit of the environment and systems occurs to prove the highest standards of practice are provided

- colleagues able to ignore their irritable behaviour under pressure. 
Expectations of nursing, continued

Expectations of the nursing by technicians

- respect and to be treated as a colleague

- to be included in in-service education to gain an understanding of operating room issues

- assistance when required

Expectations of the nursing by health planners and administrators

- adequate supply of nurses with experience or interest to learn

- quick learning in order to undertake the work required and function at full capacity as quickly as possible

- good systems knowledge and ability to maintain the workflow to achieve the 'production' schedule

- cost-efficient use of equipment and resources to ensure that expenditure is minimised where appropriate.

\section{Purpose of the nursing role}

Manage a sterile environment and plan availability of resources to receive and support the patient and health team before, during and after the operation to achieve a safe and effective outcome. The patient may be awake, sedated, unconscious or semi-conscious at any point of the interaction.

\section{Knowledge and skills required}

- Knowledge of a wide range of operative procedures used to diagnose and treat presenting health problems, their uses for different situations and patho-physiology and the potential complications that can occur

- Knowledge and application of risk management issues including ensuring that appropriate preparation has occurred including informed consent, information giving to allay fear; associated with the perioperative period including identification of allergies, associated health problems, appropriate pre-operative screening

- Skill and effective interpersonal skills to establish a relationship during a time of anxiety and sedation in order to reinforce trust in the health team

- Knowledge of the different equipment used, potential complications and how to anticipate and manage crisis situations that arise in acute and elective procedures

- Knowledge of the full range of acute health presentations and the appropriate nursing care for each, as many patients are very unwell when presenting for a procedure or recovering thereafter

- Aseptic technique, sterile draping and theatre techniques in order to respond confidently and consistently in establishing a sterile and safe environment

- Intensive assessment skills to monitor changes in a patient's condition and alert the medical and allied health team to emergency response 
Expectations of nursing, continued

Knowledge and skills required, continued

- Skilled positioning in order to minimise neurologic and tissue pressure risk

- Experience and skill in anticipating the needs for assistance and equipment that may be required by the surgeon

- Post-procedure recovery and monitoring prior to return to the ward or unit

\section{Anticipated outcomes}

- Equipment and resources are ready for the procedure including additional resources that might be needed in anticipation of potential problems

- Problems are anticipated through appropriate assessment of the patient's needs and experience of the surgeon's practice

- The patient is comfortable and supported during the procedure whether awake or asleep

- The procedure is completed without any organisational delays to minimise the risk to the patient

- The patient is not put at risk because of appropriate aseptic technique during the procedure.

\section{Actions/Interventions}

\section{The diagnostic and monitoring function}

Administering and monitoring therapeutic interventions and regimens

\section{The helping role}

Physiological (basic)

Immobility management: Combating the hazards of immobility by

positioning, splinting, transport.

Co-ordinating and managing multiple instantaneous therapies

\section{Physiological (complex)}

Drug management: medication administration. Analgesia administration in

the post-anaesthesia recovery area.

- Neurologic management: Neurologic positioning, neurologic monitoring.

- Peri-operative care: intra-operative infection control. Post anaesthesia care. Preoperative co-ordination; surgical assistance. Surgical precautions. Surgical preparation. Intra-operative temperature regulation. Assisting the surgeon and or anaesthetist with the procedures. Undertaking full assessment of the patient post procedure and monitoring patient response until transfer or discharge.

- Respiratory Management: airway management post-procedure during recovery. Airway suctioning. Aspiration precautions. Endotrachael extubation. Oxygen therapy. Respiratory monitoring. Tube care: chest. 


\section{Expectations of nursing, continued}

Skin and wound management: Incision site care. Pressure management and skin surveillance. Suturing.

Tissue perfusion management: Blood products administration. Circulatory precautions. Fluid management and resuscitation. Haemorrhage control. Assistance with intravenous insertion (peripherally and central venous intravenous catheters). Shock prevention and management.

\section{Behavioural}

Communication enhancement: Active listening.

Psychological comfort: promotion: Introduction on arrival and during checkin and risk assessment. Anxiety reduction. Calming technique. Distraction. Simple relaxation therapy. Orientation of the patient to the processes and environment where the patient is awake.

\section{The effective management of rapidly changing situations Safety}

Crisis management: setting up the environment for the management of a crisis. Sequencing and managing the logistics of rapid multiple therapies in response to a crisis. Code management. Crisis intervention. Organising the team and orchestrating their actions during a crisis.

Malignant hypothermia precautions. Organ procurement. Resuscitation. Initiating emergency procedures if required and co-ordinating the actions of the team members. Diagnosing, monitoring and managing emergent and or life threatening situations to maintain vital functions and physiologic stability.

\section{Risk management:}

- Area restriction management. Preventing hazards in the technological environment, engaging in safety work. Allergy identification and management. Aspiration precautions. Environment management: safety, worker safety. Infection control. Latex precautions. Pneumatic tourniquet precautions. Pressure ulcer prevention. Vital signs monitoring. Assessment of the patient on arrival, reviewing of patient preparation for the procedure including consent, physical preparation and relaxation due to pre-operative medication.

- Supervising the positioning of the patient on the procedure table for safety and comfort, to prevent risk of pressure. Co-ordinating the transfer of the patient from the table to the bed or trolley at the end of the procedure to minimise the potential for harm to patient and staff.

\section{Family}

Lifespan: Family support (childbirth, bereavement, and organ donation).

Providing the family with information and support. 


\section{Actions/Interventions, continued \\ Monitoring and ensuring the quality of health care practices Organisational and work-role competencies \\ Health system \\ Health system mediation: patient rights protection. Preoperative co- ordination. \\ Health system management: Physician support. Frontline quality improvement, monitoring and risk management. Minimising healthcare system failures in destabilised work environments. Product evaluation. Specimen management. Supply management. Preparation of the environment based on the procedure to be done and anticipated variance. Allocation of the appropriately skilled nurse. Monitoring the patient condition and anticipating the needs of the patient so that equipment is available for the surgeon who is concentrating on the procedure and the needs of the patient.}

Co-ordinating the actions of the other team members in the operating room, making sure that the people present have the consent of the patient to be there, are able to cope with what is happening and do not compromise the aseptic environment.

Information management: documentation. Health care information exchange. Incident reporting. Order transcription. Handing the patient over to the ward or unit staff for ongoing monitoring and treatment.

Coaching others in interpreting, forecasting and responding to patient transitions.

Building and preserving collaborative relationships.

Main taxonomy headings: McCloskey \& Bulechek (1996); Benner (1984); Benner, Hooper-Kyriakidis \& Stannard (1999).

\section{Achievement}

\section{Efficiency}

- Number of procedures undertaken, increasing in number and complexity

- Timely organisation of the department to cope with planned and unexpected requirements

- Readiness for scheduled cases and timely preparation for unexpected cases

\section{Effectiveness}

- Caring: Patient satisfaction with reception and support during the procedure where awake

- Safety: Management of potential emergencies and complications to minimise risk to patients

- Safety: Technical aspects of care: Minimal risk of pressure areas or nerve damage through careful positioning

- Teaching: Availability of information resources for ward or unit staff preparing patients for procedures 


\section{Responsiveness}

- Adaptability to changing procedures and new technology

- Ability to cope with a wide range of acute and elective presentations, unstable clinical situations and people experiencing high anxiety

- Readiness for emergencies and ability to co-ordinate the health team

In this case study nursing is shown to have a significant role in the Operating Room setting, as the leader of the health care team in the effective management of rapidly changing situations, in the diagnostic and monitoring function in pre and post procedure recovery. Nurses are key to administering and monitoring certain therapeutic interventions and regimens despite the role of anaesthetist and anaesthetic technician. The nurse undertakes a number of helping functions that assess risk and minimise harm. The nurse provides supervision of other workers and provides education and coaching to the support workers. The nurse monitors and ensures the quality of health care practices, including setting the standard, auditing standards and facilitating change of systems and processes to achieve better outcomes. A key function is the organisational and work-role competencies, which includes advanced team management and co-ordination of service delivery of the health system and use of information management technology.

In this next case study the role of the district nurse is considered as an assessor of need and co-ordinator of resources to meet the needs identified. The range of areas for involvement are broad and focus on recovery and maintenance of self-care independence. 


\section{Case study 9.}

\section{Practice Setting: District Nursing}

\section{Introduction}

The role of the District Nurse in New Zealand has altered significantly over the past decade. The role had been established in the early 1890s as a number of roles: as a visiting home care nurse for more personal care in towns, such as the Nurse Maude scheme, or the more rural role working with backblock communities to provide a broad range of environmental assessment, disease containment, treatment and home care. The Social Welfare Act passed in 1938 raised the expectation of 'free' publicly provided home care. Health planners used the role of the home visiting nurse to provide 'free' care and as a way of coping with the influx of patients who also sought free hospital care. These district nurses worked as an "extramural hospital" to ensure that patients could be discharged home more quickly. They visited people in their homes on a daily or twice daily basis to undertake a range of personal care activities, including supervision of medications, bathing, wound care dressing, terminal care of the person at home, lifting and placement of patients out of bed and returning them to bed for caregiver support. As the technical aspects of care altered, these nurses developed the skills and expertise to provide care for people of all ages with intravenous catheters, infusion pump management, oxygen therapy, tracheostomies and gastric feeding procedures. In the past decade, however, other groups have assumed a number of the functions that were considered an interesting component of the role of the district nurse. This has arisen because of changes in contracting for services by the Health Funding Agency. There have also been changes in service delivery with specialist nurses assuming care for children in some districts and specialist 
oncology teams attached to local Hospice units providing some of the care for people in their homes. These changes have raised questions for district nurses about their future role.

\section{The socio-political context}

Over the past two decades communities have been faced with confusing changes in service delivery, as the traditional services have been contracted to non crown-owned agencies rather than the traditional providers. While there was demand for improved service quality and people were increasingly prepared to pay for better quality, they were not anticipating the significant changes that occurred. There was a period of confusion as people worked out which agency was providing services and what could be acquired 'free' and what would attract a fee.

\section{Health issues}

In 1992, with the introduction of the 'purchase-provider' split in health service funding and administration, decisions were made to award some contracts to non crown-owned agencies in order to foster competition between public and private sectors (Ministry of Health, 1992). This was done in part to encourage efficiency in the public sector, which was perceived as complacent and not as customer oriented as desired. There was also political pressure from primary and voluntary agencies who wanted the opportunity to provide services, as they perceived a monopoly by public providers and thought they could provide a service more appropriate to the needs of the target group. Also services offered to children were separated from those offered to adults, because advocates for specialised child health asserted that children and their families had particular 
needs that could not be provided using the paradigm of adult nursing, especially for children with chronic health problems. General practitioners have also increasingly stated their wish for the nurses employed by their practice to provide more wound and personal care for patients. Patients have resisted this because they must pay for this personally, whereas the district nurse service is 'free'. Public service providers also modified the range of services they offered because they were not paid for some services under the Health Funding Agency contract.

Inpatient services have been required to improve their efficiency, to reduce length of stay and reduce costs. This has meant more procedures have been offered as day procedures and patients have needed more family support because of earlier discharge. Inpatient services have utilised the communitybased services to some extent, but referrals to the District Nursing Service have only increased slightly, with many patients being advised to see their general practitioner for suture removal and similar primary care needs. In-patients have also been referred to the community hospice units instead of utilising community nursing input. Tensions have arisen when the local hospice has not provided all of the palliative care but has relied on the District Nursing Service to undertake some of the functions, undervaluing the expertise of the community nurse. This 'shared care' arrangement has not always fostered consistency or continuity of care for the patient and family. The change to the role of maternity service provision has also reduced the scope of the district nurse role, as they no longer have contact with new mothers. The 'lead maternity carer' is expected to provide mother and new baby care. 


\section{Issues for nursing}

The role of the District Nurse has become more fragmented as specialist nurses, not-for-profit providers and general practitioners have perceived that they could assume parts of the role but rely on the traditional service when desired. This has made it difficult for nurses to maintain their motivation as components of the role have eroded. The district nurse role had traditionally had a total family focus and has been altered by separating out the baby and child care component. The role has further contracted, as there was less palliative care work for the dying patients in the community. Changes in treatment procedures has also meant that patients have not required home-based care in the same way after radiological, laser and endoscopy interventions. The emphasis of evidencebased practice and better scientific understanding plus new dressing treatments has also meant fewer visits. Old practices have been replaced by new dressing applications requiring less intervention and quicker healing of chronic wounds. These changes in practice have required considerable upskilling and a paradigm shift but have also reduced the workload for the community nurse.

How might the 'Contribution Model' communicate the value that the nurse provides in this situation? This will be shown in Table 16. 
Table 16. Contribution framework outlining the work of the district nurse.

\section{Expectations of nursing}

\section{$\underline{\text { Stakeholders }}$}

The patients and their families are the obvious primary consumers of the service and these include people who require assessment of their needs for personal health care in the home until able to assume self-care or no longer requiring assistance because of referral or death. Other agencies also have expectations including hospital services, general practitioners, other agencies and health administrators.

Expectations of nursing by patients

- Professional manner - pleasant, respectful of working in the patient's home, accepting of the circumstances of the patient and non-judgemental, willing to do more than the task required on referral

- skilled and knowledgeable professional whose practice is consistent with what was received in the acute hospital

- access to services and equipment through referral or assistance with applications for equipment and supplies

- timely and efficient service

- support of family, acknowledgement of their role in caring for the patient

- explanation and teaching for self-care.

Expectations of nursing by hospital services

- skilled practitioners able to care for patients with specialised needs

- ability to provide service immediately or on the next day, 24 hours a day

- communication of problems to the general practitioner and planning to access alternative care if required

- access to equipment and supplies for the patient appropriate to their needs and suitable for home use

- ability to care for heavy and complex patients with minimal assistance

- ability to understand complex family and social circumstances without being alerted to the issues.

Expectations of the nursing by general practitioners

- provision of care for the patient as directed but not assuming more care than specified. The patient must still remain under the care of the general practitioner who is to be called if required

- to be kept informed in a 'shared care' relationship, if the District Nursing Service receive a referral, and about ongoing care needs

- provision of wound care and other treatments that the patient cannot afford to pay for themselves. 
Expectations of the nursing by not-for-profit agencies

- provision of care, supplies and equipment that the agency does not provide or as a back-up for their service

- advice as required

- shared care arrangement in certain circumstances

Expectations of the nursing by health planners/administrators

- nurses with up-to-date knowledge and skills able to work without supervision in the homes of patients

- that the nurses will be aware of potential physical risk and take appropriate action to minimise personal harm and get assistance

- a cost effective service with the fewest number of visits possible and an appropriate amount of time spent with each patient

- appropriate use of equipment and resources and no more than is allocated

\section{Purpose}

Provision of contemporary nursing care in the home, appropriate to the needs of the person, to assist the person and their family adjust to their health situation safely and comfortably and assume self care as they are able.

The main areas of focus of the role are to :

- Provide support and facilitate recovery after acute stabilisation in the acute care facility. This includes personal care assessment, wound care, carer support.

- Maintaining the person with a chronic health problem in their home and supporting the caregiver, for example, the person requiring ongoing

- care and assistance after a cerebro-vascular accident, with severe arthritis, wound healing needs using shared care co-ordination

- Care co-ordination of the patient with a terminal illness desiring to die at home, and support of the family

- $\quad$ Specialist assessment of people and care planning for self-care using input from the multi-disciplinary team

- Provision of personal care in rest homes in order to maintain the patient in the home and reduce need for admission to the acute facility e.g. dying elderly needing support for terminal care

- Health promotion education to maintain the health of people in the community and minimise need for hospitalisation.

\section{Knowledge and skills}

- Knowledge of contemporary procedures, their uses for different needs, the patho-physiology and potential complications and equipment used. Knowledge of the potential complications and how to manage crisis situations.

- Knowledge of the range of health presentations and co-morbidities and the appropriate nursing care. Knowledge of treatment approaches for coexisting disease problems e.g. diabetes, cardiac. Comprehensive assessment skills: physical and psychological.

- Up-to-date experience with treatments, equipment and supplies as well as wound healing strategies 
Knowledge and Skills, continued

- Knowledge of the impact of the illness or injury on the ability of the patient to assess risk and so plan appropriate safety precautions interventions to ensure safety and minimise risk

- Ability to provide care for patients with complex treatment needs for example tracheostomy, infusions

- Ability to teach and coach patients, families and students

- Expertise in re-framing and offering emotional support as grief issues arise

- How to motivate and reinforce goals to restore potential function

- Community and inpatient resources and support services

- Pain management approaches

- Medication usage and side effects

- Emergency response

- Expertise in identifying patient/family readiness to learn and knowledge in order to make best use of their readiness

\section{Anticipated achievements}

- Number of patients seen as per referral guidelines

- Cost effective use of resources

- Resumption of self care within the anticipated time period

- Wound healing as per anticipated goal.

- Satisfaction with intervention and support provided

- Consistent progress with goals through reinforced learning

- Early identification and treatment of problems and complications

- Maintenance of a therapeutic environment

- Family confidence and involvement enhanced.

\section{Actions/Interventions}

\section{The diagnostic and monitoring function}

Administering and monitoring therapeutic interventions and regimens

\section{The helping role}

\section{Physiological (basic)}

- Activity and exercise management: Exercise promotion, ambulation, joint mobility.

- Elimination management: bowel management and training. Ostomy care. Urinary catheterisation, management and training.

- Immobility management: Bed rest care, splinting.

- Nutrition support: Enteral tube feeding and tube care.

- Physical comfort: promotion: Cutaneous stimulation. Pain management.

- $\quad$ Self care facilitation: Assistance with bathing, dressing/grooming. Post mortem care.

Physiological (complex)

- Drug management: teaching of prescribed medicines, administration of antibiotics and other medications. Analgesic administration including control devices. Venous access device management.

- Respiratory management: Tracheostomy care and teaching of patient or family about self-care.

- Skin/wound management: Amputation care. Pressure ulcer care. Skin surveillance. Ostomy care. Wound care. 


\section{Teaching-coaching function}

\section{Behavioural}

- Cognitive therapy: Learning facilitation for self-care e.g. diabetes management.

- Communication enhancement: active listening. Socialisation enhancement especially with the isolated elderly person.

- Coping assistance: Anticipatory guidance. Coping enhancement. Dying care.

- Patient education: Health education. Learning readiness enhancement. Teaching individual.

- Psychological comfort: promotion: calming technique.

\section{The effective management of rapidly changing situations}

Safety

- Crisis management: first aid.

- $\quad$ Risk management: infection control. Fall minimisation. Risk identification. Assessment of patient needs and environmental safety. Patient observation and monitoring taking appropriate action to address issues including alerting medical staff as necessary

\section{Family}

Lifespan care: Caregiver support. Normalisation promotion. Continence training. Facilitating patient/family involvement and confidence in self-care.

\section{Monitoring and ensuring the quality of health care practices}

Organisational and work-role competencies

\section{Health system}

- Health system mediation: Health system guidance. Discharge planning.

- Health system management: preceptor employee or student.

- Information management: documentation. Health care information exchange. Incident reporting. Order transcription. Referral.

Main taxonomy headings: McCloskey \& Bulechek (1996); Benner (1984); Benner, Hooper-Kyriakidis \& Stannard (1999).

\section{Achievements}

\section{Efficiency}

- Cost effective service delivery in terms of Nurse hours per patient day

- Cost effective use of resources within allocation e.g. decision about wound management products and techniques

\section{Effectiveness}

- Technical aspects of care: Range of treatments offered consistent with changing contemporary practice standards e.g. wound management products

- Safety: Problems averted/timely intervention as identified using case review

- Availability and access to care: Stated patient and support person's satisfaction with assistance and involvement to enhance confidence 
- Functional status

- Symptom control

- $\quad$ Teaching/counselling, communication

\section{Responsiveness}

- Ability to undertake whatever is required in each home

- Ability to cope with emergencies and maintain communication with all stakeholders

- Ability to assess safety (self and client) and adapt interventions appropriately

In this case study district nursing has been shown to provide a wide range of care services, some of which are under-utilised. While this role has been eroded over time there remains an important function for nursing in restoring the health of the community. The contribution of nursing in this case study is that of knowing the capability of the individual and putting resources in place to achieve the goals agreed to. The contribution is support of the person in their own home to maintain independence and promote recovery or a peaceful death. The contribution extends to that of support of the family or caregiver to maintain their role, true to the agreed plan of care and ensuring respite as required.

\section{The place and contribution of nursing in contemporary health delivery}

In this chapter I have explored the contribution of nursing as a modern profession, a profession that is diverse and specialised responding to changing technology and service delivery requirements. The 'Contribution Model' has been used to articulate how the actions and achievements of nursing contributed to health gain in the current context of practice. The case studies presented identify the similar yet diverse specialised knowledge, skills and actions required of contemporary nursing. Some aspects of practice are little different from what was expected of nurses in previous decades. Nursing has been shown 
to have adapted to the changed expectations and increased responsibility that is part of the current expectation of practice. Nurses have specialised and have a narrower yet greater depth to their knowledge and have available to them a range of treatment options to offer patients. There remains a core of general practice that is essential to the safe functioning of the services offered, that is supplemented by specialist knowledge and input so that the appropriate care is provided.

The contribution of nursing in contemporary practice is focused more on: the emotional support of the person and their experience of health or illness so that they may quickly resume self care; on risk management in relation to close monitoring and attention to potential complications; on efficiency in terms of reducing need for admission and reducing length of stay; and on management of the environment and health service delivery systems. While these functions are not new, in that nursing has always been focused on these aspects of care delivery, in previous decades treatment practices were more conservative with longer periods of bed rest and convalescence in acute care settings. While there has been a drive to reduce length of stay, the New Zealand services have been less adventurous in what they do as day cases and how early they discharge the person.

In the United States, where concern has been expressed for patient safety and health outcomes because of lack of immediate post-operative care (Gordon, 1997). Some patients are discharged home with serious medical conditions and complex treatments, often requiring specialised care. Unless referred for district nursing care, patients do not always get the care they require and families falter 
under the burden of care where home-based care services are not available or are staffed with unqualified staff. Families cannot cope as a matter of course and they need the support of nurses in the community who have a repertoire of different experiences to identify when something is going wrong.

There is a significant amount of invisible pain and suffering unmanaged post-operative pain, poorly managed side effects of medications and other treatments, anxiety because family members cannot adequately assess and monitor their loved ones, complex medical conditions or utilisation of high-tech medical equipment (Gordon, 1997, p.259-261). There has also been a shift of responsibility and actual work from the doctor to the nurse, sometimes planned and sometimes not.

While this may be similar to the experience of nurses in previous decades, the reality for contemporary practice is that more and more is expected in an environment of consumerism, there is legal challenge and increased accountability. Because of the reduced length of stay and more complex technical procedures, there is less time to relate to the patients and their family, less time to deal with the higher turnover of patients and less time to assess and treat them before they are discharged home. There is less time to form relationships and establish the healing relationship. Despite this, there is acknowledgement of the value of the nurse: "anyone who gets a major illness that requires ongoing care quickly discovers one of the health system's most important groups of caregivers: the nurse" (Mann, 1998, p.38-39). 
The case studies in this chapter identify the expectations, actions and achievements of nurses in these specialist settings as they undertake a range of activities to meet the specific needs of patients. The 'Contribution Model' profiles the actions of the nurse in response to the expectations against the backdrop of the socio-political context. It is clear that while a number of the specialist groups question their future role and function, they currently fulfil a valuable function in the provision of safe and effective patient care. The contribution of nursing in contemporary practice is essential to the safe provision of health services. The nurse is the assessor of need, monitor of recovery, the provider of care, the co-ordinator of care, the manager of the clinical care environment. Nursing is the 'glue' that binds the health team together around the needs of the individual, family and community. Nursing is the face of comfort and caring, the sense of continuity and consistency.

\section{Nursing: a profession regaining confidence in the current environment.}

"Nursing may be the oldest art, but in the contemporary world, it is also one of the most invisible...one of the most invisible arts, sciences and certainly one of the most invisible parts of our health care system" (Gordon, 1997, p.ix). For many families of patients receiving skilled nursing care, they experience "firsthand the value of nursing and yet often do not 'get it'... they understand the kindness but do not understand the complexity of nursing work, the intricate weave of the tapestry of care" (Fagin, 1997, p.xix). On one hand nurses have always been the most visible providers in the delivery system (Kelly \& Joel, 1996, p.12), yet is also the most invisible as the result of many factors. This may in part be because nurses are always present, maintaining order and organisation so that it all appears so easy and 'just happens'. In this section, nursing is 
challenged to make visible the invisible and to find a language to articulate the difference nursing makes.

The invisibility of nursing practice may occur because of the nature of the work of nursing, the privacy and intimacy of the work that is done. It may be that the subtle assessment and co-ordinated management of care and the focus on empowerment of the patient and their family for self-care coaching is so practised as 'skilled know how', that the complex appears simple and achievable rather than important and intrusive. Managers may be misled by the apparent simplicity that nurses create to assist patients and families achieve selfcare, perceiving that if the family can do it then health services do not need nurses. The actions of empowerment can appear disarmingly simple.

Many health service managers, some of whom have been nurses, disappointingly fail to acknowledge and value the importance of nursing in the healing relationship, the comfort and skill in preserving personhood in the face of pain and extreme breakdown. Some service managers perceive that much of the work of nursing can be broken into tasks and delegated to less skilled workers. They fail to understand the importance of the nurse to patients and families who need guidance through emotional and developmental changes, as they integrate the implications of illness and recovery into their lifestyles. There is skill in teaching and coaching as the nurse assesses the patient's readiness to learn. The skill of nursing is often invisible in the detection and documentation of significant changes in a patient's condition, in providing early warning and skilled performance in emergencies (Benner, 1984). 
The invisibility of practice can be attributed in part to the inability of nurses to articulate what it is they do and to document it in a meaningful way. There is some urgency for nurse leaders, physicians and hospital administrators to reevaluate the functions and importance of nursing and to assist in clarifying the importance of nursing work to the achievement of anticipated outcomes. Unless the profession is "recognised and rewarded for its contribution by all sectors, nurses will continue to grapple with their sense of self-worth, identify and commitment" (Benner, 1984, p.198). Unless this is done the significant risk for the health service in relation to recruitment and retention of skilled practitioners, may become increasingly acute. "According to the American Hospital Association, nurses provide 95 percent of the care a patient receives while hospitalised" (American Nurses Association, 1998, p.2); we therefore cannot afford to undervalue the expertise and importance of this workforce in health care provision. Inadequate retention may have a significant impact on the ability of hospitals and community services to achieve the outcomes required in contracts.

Despite nursing in New Zealand having been present for more than a century of service and having made a significant contribution, I have observed that the most persistent question is still 'What is Nursing? Does nursing have a future?' (Rogers, 1972, p.42; Russell, 1991; Hayne, 1992; Wilkes \& Wallis, 1993; Crosson \& Robb, 1998;). This continuous focus reflects a lack of consensus as to the definition of nursing and a lack of focus about the place of the profession in health service delivery. Nurses agree that the "profession must define what it is that makes it important or succumb to attack" (Boylan, 1992, p.28). By this 
we mean that nursing needs to be proactive in communicating why it is required as a practice discipline and why unskilled worker cannot provide the holistic care through a series of tasks. The profession needs to take action in its own right, rather than to wait for someone else to take a lead.

The professional lament, that 'the profession' should take action to define what makes nursing essential to health service provisions raises the question: who are the 'someone's' who should or will do it? While a charismatic leader has a place in changing history, the 'someone' in this case is not one individual but the collective that is the profession. It does require some co-ordination, however, and that is probably the responsibility of the leaders of the profession internationally. The practitioners at the 'front line' look to the professional leaders to provide leadership, to create opportunities, to say what needs to be said at the right place and the right time. It is my view that the 'who' must be resolved quickly as the imperative is that there is some action. In an environment that is seeking to blend the professions and erode the distinctiveness of health professional roles, nurse leaders must take action to articulate the unique contribution that nursing makes or face a gradual erosion of the value of the professional input. The strategies posed by working groups in the United States and United Kingdom (PEW Commission, 1998; Cochrane, 2001), seek to

create a health workforce of the future with a generic healthcare worker/professional by promoting overlapping practice/scopes and the creation of a super board under the banner of interdisciplinary practice. There is a move to eliminate exclusive practice domains and promote institutional certification/licensure. There is promotion of increased use 
of unlicensed assistive personnel and the standardisation of competence. The dilution of representation by members of the professions on the super board and increased representation from the healthcare business sector resulting in increased power and control by the healthcare industry over public policy and practice authority (Californian Nurses Association, 1999).

The issue for nursing in New Zealand, is to find a unified voice to articulate what is nursing is, that is if the nursing leaders in the different groups can agree. Nursing has a professional culture (English, 1993; Barker \& Reynolds, 1994) of criticising versus critique, of suspicion about the motives of other individuals and groups within their own profession. Those that take initiative are criticised rather than supported and acknowledged. In New Zealand we call it 'the Tall Poppy Syndrome', the 'de-heading' of those willing to take a lead. What this cultural behaviour does is maintain the status quo, fosters the mediocre and constrains innovation and risk-taking. It would be interesting to observe the actions of the profession if an individual or a group were to take a lead. Would the profession celebrate, co-operate, participate and use the initiative as a stepping stone into the future?

In lieu of a shared leadership response, actions need to be taken by the current practitioners as people in positions of daily practice and leadership, who are able to influence what society thinks of nursing. It should to be practitioners able to negotiate the 'what' that communities and health planners want from nursing, so that the profession can design the 'how' of service delivery. It needs to be contemporary practitioners agreeing how nurses are prepared for entry to the 
profession and post-registration to meet the range of health needs. It needs to be communicated through practitioners who are able to create the welcoming, coaching and valuing teamwork environment within which nurses want to work. The profession has already been challenged with the words "if we're the ones who can imagine it, if we're the ones who dream about it, if we're the ones who need it most, then no one else can do it, we're the ones" (Styles, 1982, p.5). The call to action requires a shared image, a description of nursing and its contribution that encapsulates the essence of the art and science of nursing practice.

There is a need for assertive action by the profession as a whole rather than waiting for 'someone' to take action. The danger of waiting is that 'windows of opportunity' pass and in the absence of proactive action, alternative workers are introduced to replace parts of what registered nurses do. Nurses are being replaced, nationally and internationally, by untrained staff and their scope of nursing practice extended in scope to replace junior medical staff roles (Fagin, 1997, p.xix; Conroy \& Cochrane, 1996; Conroy \& Cochrane, 1999; Cochrane, 2001; Royal College of Physicians, 2000). Nurses are forced to defend their function in the health team, to find language and descriptors of practice to establish their importance to health outcomes. The diversity of function of nurses in contemporary practice makes one description of function difficult. Nurses can be identified as a professional clinician, a knowledge worker, whose role complexity and responsibility require long-term and continuing development, not short-term reactive realignment of tasks and functions. The development of expertise in nursing for proficient and expert practice requires time, practice and supported development. The profession should have a strong image of its 
contribution, of where nursing fits into the whole of health care as an integral component of the interdisciplinary health team. Each discipline is present because of its distinct and specific contribution and should not waste energy on continuous defence of their right to 'be present'. The dilemma for nurses over many years has been that "nursing cannot be explained by conceptual jargon. If we are confused, it may be because we are swayed by the demands of academe for pomposity; of management for simple explanations and rules; of professional colleagues for obsequiousness; of a sexist society for conformity" (Diers, 1984, p.204). Over the past century the discipline of nursing has applied the knowledge of nursing in its broadness to many contexts, adapted new technical knowledge to the situation and in so doing maintained an evolving scope of practice that almost defies definition.

The current face of nursing is of women and men who positively provide a broad scope of increasingly complex professional practice, in a wide range of settings, experiencing increasing pressure to perform. While nursing has traditionally been sustained by the intrinsic reward of work amidst camaraderie and community appreciation, there remains an undercurrent of discontent and a desire for 'more' expressions of 'valuing'. Practitioners want to be acknowledged for the pressure they face daily: as consumers demand more and as practitioners deal with an increasingly technological and legally complex environment. Recent health service changes in New Zealand and requirements to justify cost-value of practitioners in a system experiencing strain, have highlighted the fragile psyche, the lack of professional infrastructure to provide the positive, proactive leadership. In addition to uncertainty about job security, nurses are working longer hours of work; they report highly stressed environments and concern over 
the quality of care they are able to provide (Buerhaus \& Staiger, 1997). While nurses themselves characterise their work as indispensable to society, there is acknowledgement that it is neither understood and is not given due recognition by society. Nurses (internationally) wonder whether they will find the fulfilment necessary to bring a measure of stability to break the vicious circle of staff shortage (Parrot, 1973). Nurses want and need strong decisive leadership and advocacy as they survive the clinical practice reality. They are aware of the short and long-term workforce shortage issues and the increased demand for nurses that is contrasted with a decline in the supply (Hinshaw, 2001). Most do not, however, have the time, energy or expertise to undertake advocacy on their own. They need leadership that is clear, coherent, consistent and 'catchy'.

The desire for expressions of 'value' is not new, nor is the passive waiting for others to see the importance of the contribution. Nurses through the decades have been exhorted to influence their current and future reality and not wait for leadership. Nurses have also been prompted to acknowledge the positive and accept responsibility for creating their own destiny.

" A brief glance at the history of nursing progress in New Zealand will show how singularly fortunate we have been in the organisation of our profession, but the result is that we have developed a tendency to sit back and wait for someone else to take the initiative. The time has arrived when we should be fully awake to our civic and professional responsibilities... what changes will take place in the social structure which will affect nursing and how can we best equip ourselves to meet these changes. What changes in the medical and nursing services to the community would 
nurses, in light of their experience, consider desirable - what fresh fields do they consider should be open to nurses - what training would be necessary to enable them to undertake increased responsibilities?"... "We must look forward, thinking and planning for the future (Kai Tiaki, 1943, p. 198).

This excerpt from the 1940s challenged the profession to view their place positively and to be more outward looking. This same exhortation could be voiced to nurses in the current decades, seeking their commitment to seek new opportunities and possibilities rather than remaining 'exhausted' by the demands and fearful of the expectations and changes.

There is a view within the profession that nursing has been generally passive, assuming continued community support, rather than using its influence and intellectual capability to examine the changing health care setting to promote a dynamic image of nursing. Williams (2000) suggests that in fact developments in nursing in New Zealand, over the three decades from 1960, indicate a strong sense of self-determination, willingness to take initiative, to specialise, to try new approaches in care delivery, to establish independent practice. While this is a positive extraction, nursing could also be viewed as 'a profession directed'. Florence Nightingale made a significant impact on establishment of nursing as it has over the past one hundred and forty years, in establishing nursing standards, education and promotion of the potential of nursing. She raised social expectations of the importance of skilled nursing practice (Keith, 1988; Wood, 1994). The influence of medical science on nursing development has been similarly considerable. Nurses have accepted teaching from medical staff and accepted more delegated tasks from medical practitioners as science and 
technology has developed. Nurses have sought sponsorship and advocacy from medical staff and specialised in the scopes of medical specialisation. The decision by the profession to change from apprentice-type preparation to college-based learning could be viewed as an attempt to mimic the preparation of other professions as a way of enhancing credibility or to remove the profession from the 'hand-maiden' image to that of an educated profession. Regardless of the perspective, the profession has generally waited for leadership, those intrepreneurs and entrepreneurs a small cohort internationally.

There is no doubt that nursing, as a subsystem in the wider system of health and society, has been and continues to be influenced by these factors. The profession has, however, used these influences to progress and has shown adaptation and used cumulative experience and new knowledge to achieve a diversity of practice profile previously not possible. Nursing has developed skills and experience in specialised practice roles to reinforce the traditional and has created a new place for nursing in the changing provision of health care. The profession has also remained connected to the community it serves, not moving as quickly as some would desire but remaining connected to the communities with whom the profession has a social contract.

The development of confidence over the past decade has shown practitioners using evidence-based practice, undertaking research and contributing to scholarship, negotiating service contracts, assuming advanced practice roles, continuing their education and contributing to policy debate. These developments reflect a profession still willing and able to contribute to contemporary health 
care delivery. The increasing celebration by the profession of the achievements of those who have become entrepreneurs indicates that modern nursing is regaining its confidence to articulate its importance and value.

\section{The modern image of nursing}

Nursing is characterised by its diversity at all levels of health service delivery: primary, secondary and tertiary. Nursing is practised in all settings: in general medical primary practices; in independent nurse-led practices; in schools, industrial workplaces, hospitals, community facilities and government administrations. Nurses are in direct contact for periods of time with all strata of the population at large, the main connection between the individual and family, other health professionals and community agencies. Nurses have assumed new proactive roles while still performing a wide range of similar activities as in the past, demonstrating that they are practitioners capable of confronting new situations, who can anticipate and influence the health care for communities (Labelle, 1985).

The public image of modern nursing is influenced by romantic memories of the heroic nurse of the past and the reality of the nurse in current practice. Nurses of the past are depicted in misty paintings or photographs, ethereal, beautiful, in a perfect white uniform, more a fantasy than the reality. For many of the current elderly consumers, this image of the nurse at the bedside of the injured soldier at war is a real and relatively recent memory. Over 50,000 nurses world-wide travelled during the Second World War to Europe and Africa, to provide support, care and comfort to the many badly injured soldiers and civilians. These images of the role of the nurse were reinforced in the popular television programmes of 
nursing in army hospital units and emergency department television dramas. There are other personal images of the nurse comforting a crying child, providing attention to the individual and their family as they deal with the injuries, shock and impact of a major accident. Individuals have memories of the nurse being the caring and supportive health professional with a person with horrific burns, or caring and mobilising the elderly or dependent person.

What is rarely visible, remembered or reported is the nurse assisting someone who is struggling to breathe, the nurse calming someone experiencing a psychotic event, the nurse working with a family struggling to acquire resources and regain self-esteem amidst extreme poverty or violence. While the positive heroic depictions have a place and reflect one reality, the other images rarely remembered reflect nurses as uncomfortable reminders for patients of their vulnerability and "for physicians of their fallibility" (Fagin, 1997, p. xix). New nurses also report that their friends, the public, also "think what I do is demeaning" (Benner, 1985, p.198). What is not visible or understood by the public is the range of skills required to undertake nursing in a skilful manner. What is not articulated or explicit is the critical thinking and problem solving requirements, the resource management skills and the requirement for flexibility. What is not appreciated is the leadership ability, supervisory skills, the delegation skills, the team-building and teamwork skills. What is taken for granted is the importance of the depth of knowledge of health care systems for consumer advocacy and case management. What is assumed is the good written and verbal communication skills, the professional orientation, the clinical experience, good assessment skills, good technical skills, change agent and information systems confidence. 
Nurses asked what "Nursing is" generally provide descriptions of tasks or broad generalisations, preferring to describe events and examples of their work. In reality the work of nursing is complex and diverse, involved with individuals and groups over long or short periods. Nursing deals with a wide range of health problems that may result in recovery, may require assistance to adapt to chronic health and disability or need care until death. Most often the work and relationship is ongoing, it involves sad, stressful and traumatic events, personal moments of incremental achievement and private experiences of extreme joy and sadness. The work may involve protecting patient safety while the person is unaware of their environment either because of unconsciousness or during a psychotic episode. The practice of nursing occurs from a broad base of biopsychosocial knowledge and practices, is comprised of multiple possible treatment and intervention options that can be used to meet the diverse and multiple needs of people and their families through the age span. Nursing occurs in numerous settings: in hospitals where people present for treatment of asthma, pneumonia, strokes, diagnosis and treatment of cancer, present for surgical intervention, for treatment of injuries, burns and for rehabilitation - every imaginable health problem. Nursing occurs in homes and in the community with the involvement of district nurses, public health nurses, plunket nurses, community mental health nurses. Nurses provide assistance, treatment and support as people assume self-care and independence. Nursing occurs with assessment, treatment and monitoring of communicable diseases such as meningitis or tuberculosis or sexually transmitted diseases. Nursing occurs with refugees, with new immigrants, in schools, in factories and workplaces. Nursing occurs in prisons, in boarding houses, in caravans, under bridges, with homeless 
who are mentally unwell and in self-help community settings. Nursing continues to go to help in times of war, provides care in epidemics and natural disasters. Nursing is everywhere, using a broad base of knowledge to meet whatever is asked.

The actions of nursing are multiple to achieve the outcomes expected. It is difficult to explain in one sentence what nursing is, its importance and what it is that nurses contribute. The 'it' may not be an action that can be observed or formally evaluated but rather the importance of being present with empathy, comfort and confidence. Nursing, as a practice-oriented profession, has not generally focused on one episode of care as midwives do, has not specialised as medical practitioners do on the diagnosis and cure of particular medical and surgical disease groupings. The practice of nursing has allowed practitioners to respond to most situations and to provide care for a range of health needs in a comprehensive manner. It is the very diversity of practice settings and the many faces of nursing that have created the lack of identity for nurses and the confusion for the community who see nursing as being 'all things' and do not have a simple clear description that is easy to use.

Having a clear description of nursing is essential, not just for the understanding of consumers of the service but also for nurses themselves, as many nurses seek a statement of purpose as an important indicator of their identity and ownership. For some professions a descriptor is relatively simple, such as 'diagnosis and cure' for medical practitioners or 'management of pregnancy until establishment of the family unit' for midwifery or 'assessment, diagnosis and treatment to regain functionality' for physiotherapy. For nursing there does not appear to be a 
single descriptor of function or outcome. Rather nurse theorists offer diverse suggestions such as the nurse does for others what they would do for themselves if they had the strength, the will, and the knowledge... and makes the patient independent of him or her as soon as possible (Henderson, 1966); or the role of nursing is to use their abilities to prescribe, design and provide nursing for legitimate patients by performing discrete actions and systems of actions that engage the individuals capabilities to engage in self-care and meet the self-care requisites of the individual therapeutically (Orem, 1988); or the goal of nursing as to facilitate optimal wellness through retention, attainment, or maintenance of client system stability by means of primary, secondary, or tertiary prevention (Newman, 1986).

While these and other similar descriptions by nurse theorists go partway to describe the purpose and actions of nursing, nurses do not easily use these descriptions to explain their role and function to consumers. Other descriptions in the literature attempt to provide a systematic description of nursing practice as “... assisting individuals or groups to maintain or attain optimal health, implementing a strategy of care to accomplish defined goals and evaluating responses to care and treatment" (National Council of State Boards of Nursing, 1994). Again this definition does not detail fully what is unique about nursing.

My personal description of nursing is 'nursing is the skilled use of specialised knowledge and practices to assess and respond to the identified health needs of communities and individuals (whether healthy, unwell or dying), to assist them to achieve their goals and potential for health gain'. This reflects my perspective of the general purpose or mission of nursing, namely to help people identify their 
needs and achieve their goals and outcomes using a range of techniques and practices as appropriate. This may still not provide a unique descriptor but allows the outcome of the nursing intervention and contribution to be measured, as the achievement of goals, whether they are to regain health, to resume a 'normal' life in the community or to die peacefully. Definitions of nursing are by necessity generalised, given the diversity and range of interactions possible. Whatever definition is used, it requires that nurses identify the needs of those they work with and respond to the context and customer group to develop a more individualised plan for intervention.

Nursing has over the past two decades used narrative as a formal method of placing practice within a context to explicate the unique contribution (Benner, 1984). This method has encouraged nurses to write about situations in their practice that were important for reflection on the decision-making and interventions in their practice and for the patient outcome. It has been uncomfortable for many nurses to claim the importance and achievement of their intervention and many have been inexpert in the identification or evaluation of achievements. They have, however, provided useful insights into the private world of practice. More recent descriptions of nursing interventions have been shown to provide order in the descriptions of practice although the process of classification is achievable but remains cumbersome (McCloskey \& Bulechek, 1996). Classification lists of actions, however, have limited value if not linked to a model or framework that acknowledges also the context, expectations and links actions to achievements. The integration of the narrative, classification and descriptors of competence can enhance understanding by communities, managers and the profession of the value of nursing contribution. 
Practice has changed over time from an accepted general practice knowledge to a more specialised knowledge responding to technological advances. At the beginning of the twentieth century emphasis was on acquisition of a broad knowledge base to respond practically to any situation and to be ready to go anywhere. Current practice requires a mixture of generalist knowledge and application and increasing specialisation as developments of new knowledge require specific learning for special application in some settings and for some specific groups. The challenge for nurses as professionals is to accept that the career of nursing will require continuous upskilling and retraining in speciality practice as technology changes the expectations of health service delivery. Practice has become more specialised from the late 1970s as medical specialisation and technology has targeted treatment practices for specific disease processes. The focus of nursing as a general practice, able to respond to any disease or health presentation, has narrowed. The degree of specialisation has made deployment more difficult and has meant that certain groups of specialists are in higher demand in in-patient settings. Nurses working in intensive care, cardiac and neonatal intensive care for example, are sought after internationally because the work is very technically specific requiring considerable education and experience to ensure safety. The demand for skilled specialised mental health nurses has continued despite a perception that generalisation and comprehensive practice would be required of the 'nurse of the future' (Carpenter, 1969).

What has not changed is the central practice of nursing care: in care co-ordination of the multidisciplinary team; in the management of the environment to reduce cross infection; in the role of the nurse in providing physical comfort care 
practices; and in the supportive relationships between the patient and the nurse that allows goals to be identified and achieved. Many of the specific tasks of practice, however, may have changed due to the increasing use of evidence-based practice. The function of nursing in these areas has remained constant. Nurses have continued to work across the twenty-four hours and seven days of a week, to lift, turn, wash, respond to changing needs, deal with emergencies and routine requirements. Nurses have continued to deal with life and death, assisting people deal with the impact of these events on their lives. Nursing work has always been dangerous, dealing with people who are angry and stressed with what is happening to them and their lack of control over events. The experience of violence in the workplace is receiving greater publicity as people speak out about groups in society who use anger to express their needs and as more people present having used drugs and demonstrating reduced social inhibitions.

What has changed in nursing are some of the techniques of nursing practice, altered because of changes in health care technology, in research and the tertiary preparation for practice. There has been increasing specialisation in practice, nurses working in independent practice and increasing numbers of nurse specialist roles working to co-ordinate the care of people with lifelong health problems such as diabetes, chronic airways disease, chronic renal disease, asthma and pain management. Change has occurred in response to technological advances, such as the use of laser surgery, new medicines, use of prostheses for fractures and other problems, use of radiological diagnosis and interventions. These technologies have altered the type and duration of patient stay, increased day procedure treatment options and shortened in-patient stay, with different procedures and less need for invasive procedures. More nursing care is provided 
in the homes of local communities, with 'hospital in the home', safe technology for infusions and other treatment processes. A number of large mental health hospitals have closed in favour of smaller acute wards on general hospital sites, with more people being cared for in the community, using nursing case management and respite care. This has impacted on the work of psychiatric nurses, with the establishment of crisis teams and the work of nurses as 'Duly Authorised Officers' able to assess and arrange admission for clients requiring acute assessment and care.

These changes in the roles and responsibilities, with increased autonomy for nurses, have raised issues about liability, risk management, inter-disciplinary practice and the need for post-graduate education. While some practices may have changed and areas of speciality have developed, the fundamental needs of people have not changed and the practice of nurses in identifying need and planning for provision of clinical care has not changed. Nursing continues to be in high demand to meet the health needs of the community. 


\section{Chapter Seven}

\section{Nursing contribution into the future}

This chapter considers the role and contribution of nursing into the future, using scenario planning to consider a variety of possible futures within which nursing might be asked to contribute. The application of the 'Contribution model' considers the general descriptions of practice across the health service similar to Chapter 5, rather than focusing on individual roles and scopes of practice as was the approach in the previous chapter. The implications of the scenarios, developed instead of case studies, will be used to generate some recommended strategies that could be relevant for action by the profession. It is critical that the profession influences the direction of its own future contribution where it can, while remaining responsive to the needs and expectations of the community served.

"Our mental models or paradigms shape how we perceive and act, the ultimate dilemma being that all our knowledge is about the past yet all our decisions are about the future" (Wilson, 1975). This perspective is highly relevant for the nursing profession as it grapples with the challenges in the present and considers its place in the future.

The previous chapters have discussed some of the influences that have impacted on the mental models that have shaped nursing as it is today. The single consistent characteristic through the discussion has been the responsiveness of the profession to the changing needs and perceived expectations of society in which nursing has worked. The function of being 
responsive acknowledges connection and adaptability. Passive acceptance, however, of the complementary nursing role in the health care team could be perceived as acceptance that others will decide what is expected of nursing and about how health services will be shaped.

While nurses have been invited to become more involved in planning for the future of the profession (Ministerial Taskforce on Nursing, 1998; Nursing Council, 2001), generally only a few interested parties make responses during consultation exercises. Those responding are willing to communicate their views to shape the future direction of the profession but they may not reflect a true sample of professional knowledge and intellect, or the will of the profession. In order to move from passive to active participants in shaping the future, it is essential that a methodology be found that can challenge our 'mental maps' and generate thinking outside of the box of 'current wisdom'.

\section{Scenario planning}

Scenario planning is a discipline that fosters entrepreneurial and creative foresight when considering contexts of accelerated change, greater complexity and genuine uncertainty (Wilson, 1975). It derives from the observation that, given the impossibility of knowing precisely how the future will play out, a good decision or strategy to adopt is one that looks at a number of possible futures. In generating "what if" scenarios, information about what is known, surmised or speculated from postulated trends is used to write a story of a possible future situation. Scenarios are tools for ordering 
ones perceptions about alternative future environments, created in plural, as markedly diverse carefully constructed set of stories about the future are built around carefully constructed plots, each one modelling a distinct, plausible world that might someday occur. Stories can express multiple perspectives on complex events and scenarios give meaning to these events. Scenario planning highlights large-scale forces that can be anticipated to push the futures in different directions. The process makes the forces visible so that if they happen they can be recognised. Ultimately the process is only worthwhile where it is more than developing coherent, imaginative and useful scenarios but is used to translate the scenarios into decisions and strategic action, to change our mental maps of the future. "Although predicting is perilous, not predicting is even more perilous. It leaves us unprepared for the changes going on right under our noses, confronts us with recurrent surprises, and most problematic, makes us reactors to change instead of agents of change" (Kassirer, 1995).

Scenario planning encourages strategic thinking as a way of learning from experiences in order to accomplish a vision. Starting at the current experience, the participants monitor what is happening to redefine and reframe a changing situation. Knowledge expands from what is learned and is applied as participants think about things differently and translate thought into action to expand their future possibilities. Strategic thinking connects the vision to real issues, interests and opportunities as they arise. Environmental scanning becomes a catalyst for envisioning which driving forces could have the most impact. Futures learning, as a methodology, has evolved over the 
past three decades in response to limitations in the cause-effect or singlepoint forecasting of future planning (Fahey \& Randell, 1998). The process utilises structured brainstorming and discussion processes as participants seek to understand the present and past by describing a range of contexts. They are asked to describe a variety of potential futures using an understanding of predetermined events and identify critical uncertainties: 'better', 'worse' or 'radically different'. The exercise delineates how such futures or end-states might evolve and what would have to happen for each end-state to occur. It also identifies what could have a substantial influence, identifying appropriate indicators to track and analyse the events, decisions and circumstances that develop in order to be prepared for whatever eventuates (Fahey \& Randall, 1998, p.193). The approach guides the learner to think strategically and consider multiple options that are particularly relevant to the area under study.

Scenario planning begins by identifying the focal issue or decision that will be used as a test of relevance through the scenario-making process (Wilson, 1975; Fahey \& Randall, 1998). The next step is to identify the primary 'driving forces' at work in the present using a future backward and future forward approach, such as the social dynamics: including quantitative and demographic issues as well as issues of values, lifestyle, demand or political energy; economic issues, the macroeconomic trends and forces, microeconomic dynamics and forces at work; political issues including electoral and regulatory; technological issues including direct, enabling and indirect. Some of the driving forces will be outside the control of society or 
the profession and be consistent in any story that might be thought through; for example, the demographic changes over the next ten years are generally predetermined now. The list will include uncertainties, some of which are critical and will be key to the focal issue. The list can be simplified into two orthogonal axes in order to define a matrix of different, plausible quadrants of uncertainty that can be used to explore a logical future. Returning to the list of driving forces, the next step is to describe the scenarios using the main elements, creating a variety of potential futures. The scenarios provide a general description of what is plausible, not always neatly fitting into 'good' or 'bad' worlds. The scenarios can then be used to postulate about how such futures might evolve given the uncertainties and what they mean so that resilience can be built into the strategy through contingency planning. Appropriate indicators can then be tracked to alert to a change in direction or transition into a possible future. Building scenarios give people experience in thinking of alternatives. Outlining the best case, the worst case and the most likely scenarios gives an opportunity to reflect on which cues could become important signals for course corrections. The importance of scenario building is not to choose the "correct" scenario and focus on it, but rather to break out of the rigidity of planning for only one preferred outcome. Thinking strategically opens the possibility for new alternatives, rather than trying to follow the previously chosen course even when alarms should be ringing. Using tension constructively to move toward finding a solution, defining how one opportunity or problem is connected to other issues and integrating multiple perspectives, all help develop an ability to respond to changes instead of simply reacting when situations don't play out as planned. 
The process of scenario planning can be depicted as shown in Figure 6 (Fahey \& Randall, 1998, p.270, 297).

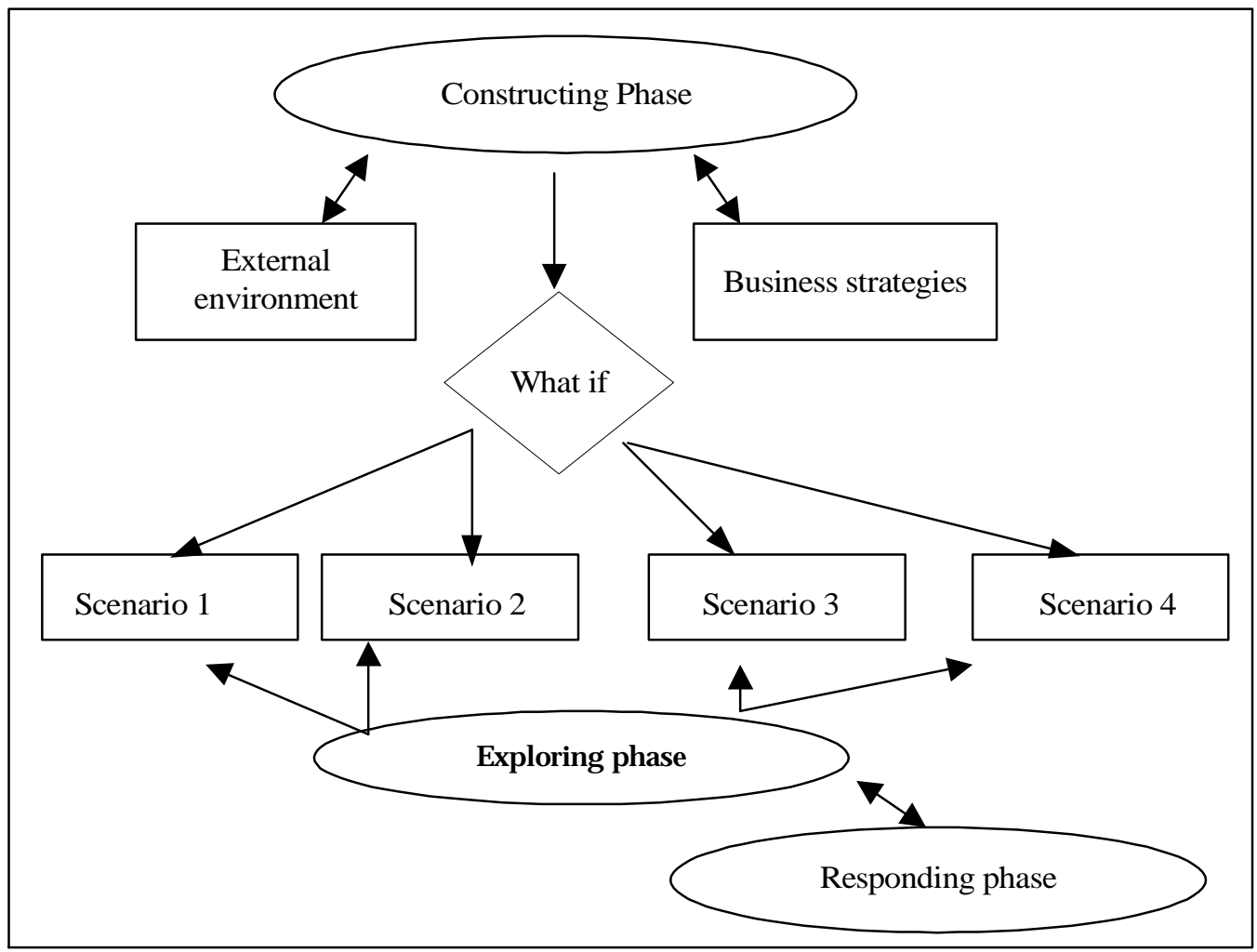

Figure 6. Scenario development method

\section{Creating a scenario: the present leading into the future}

The focal issue for discussion in this chapter of the thesis is simply: What could really happen in the next twenty years and what will the nursing profession do to contribute to the health of New Zealand in any of the possible futures? In order to answer these questions it is essential to understand the driving forces at work in the present that will shape the future. This will be discussed against the backdrop of the categories of social dynamics, economic issues, political issues and technological issues. 


\section{$\underline{\text { Social dynamics }}$}

New Zealand society has over the past decade has undergone significant change. The society is a more multicultural society. About four-fifths of New Zealanders are of European origin, predominantly from the British Isles and from the Netherlands, Yugoslavia, Germany and other nations. The indigenous Maori population comprises 14.5 percent as at 1996. Pacific Islands people comprise 5.6 percent with immigration from the South Pacific continuing in smaller numbers than migration from Asia of more Asian cultures and arrival of more refugees from diverse cultural groups over the past decade. The number of refugees has increased with the various conflicts internationally including people from Indo-China, Chile, East European, Assyria, Ethiopia, Bosnia and Somalia.

The population in 2001 is currently 3.8 million, projected to pass the four million point within the present decade and then to peak at 4.6 million at 2044 to then decrease slightly to 4.49 million by 2051 . Demographic changes over the past years include shrinking family size, increased divorce rates, increased de facto unions and an ageing population. It is projected that the future, with low birth rates and the high proportion of older New Zealanders, could herald a time of slow or nil population growth rates (New Zealand Yearbook, 1999). The number of people aged sixty-five and over has doubled during the last fifty years and will double again over the next halfcentury to comprise 26 percent of all New Zealanders compared with 12 percent in 1999 (Statistics New Zealand, 2000). 
The New Zealand labour force is projected to grow but at a decreasing rate from 1.88 million in 1996 to 2.24 million in 2019 and then drop to 2.11 million by 2051 . The male labour force is projected to grow by 14 percent and female labour force by 11 percent. The labour force is ageing with half of the labour force projected to be older than forty-two years by 2051, compared with the median age of thirty-seven years in 1996. The age structure of the labour force will also undergo significant changes with fewer young people than in the past, with only 12 percent of young people of eighteen to twenty-four years compared with 16 percent in 1996. The middle aged working group will reduce from 49 percent in 1996 to 39 percent in 2051, with the older labour force increasing from 28 percent in 1996 to 40 percent in 2051. If migration gain continues at 10,000 people per year, this would impact on the labour force with 22 percent more people than in 1996. Full-time employment has increased to 7.9 percent and part time employment to 42.8 percent.

Currently, the unemployment rate is reported at 7.2 percent of eligible employees, which is lower than the OECD average. The average weekly income for European workers is $\$ 470, \$ 386$ for Maori and $\$ 346$ for Pacific Islands people (Statistics New Zealand, 2000). With pay gaps existing between groups, wage inequality and factors drive the wages of high and low skilled workers apart. There are more women in the workplace, more parents in the workforce and more single parents working to sustain the family income. More workers have responsibility for elderly or disabled family 
members and need options for care of these dependants. Families have less time together as parents are working more. There has been a shift in employment opportunities from manufacturing to service work opportunities. The virtual market has altered the viability and marketing of goods through use of the internet and television and telemarketing.

Since the 1980s there has been increased consumerism in New Zealand as people have required better service, more choice, information and opportunity to decide for themselves what they would like. This has increased the requirement to provide justification of actions, expectation of increased access to developing technology, expectations of rights being met, choice in relation to access to health care and care providers and growth in the private health sector. User charges have been introduced where previously many services were considered 'free' as part of the social welfare state such as education, social welfare and health. There has been increasing discussion about rationing of resources and services based on best return on investment and benefit. There has been increasing restitution to Maori for historical land acquisition that has potentially increased the wealth of this group allowing for greater self-determination.

Changes in eating habits have occurred with more people eating in restaurants and cafes and more people classified as overweight although a significant number strive for more healthy living and self-care with reduced consumption of fats and other foods. The commercial sector has extended the hours of service as more shops are open seven days per week and are open 
longer hours across the whole year, which is similar to international practice. The pace of economic reform in New Zealand over the past decade has had an impact on social structures for those in the population who are less well off (Kelsey, 1995). The situation of poverty has resulted in poor nutrition in certain groups, with more people experiencing malnutrition by either being over or underweight. The level of violence, abuse and anxiety has increased in line with global trends, with convictions for violence increasing from 9.4 percent in 1992 to 13 percent in 1997. New Zealand has an inmate population of 12.9 in prison per 10,000 mean population (New Zealand Yearbook, 2000). International comparisons have also identified that New Zealand has one of the highest rates of suicide in young people among developed countries (World Health Organisation, 1996).

The suicide rate for young males aged fifteen to twenty-four years is 40.9 deaths per 100000 in 1997 (New Zealand Health Information Service, 1999). This is significantly higher than corresponding rates in recent years in Canada (24.7 per 100 000), the United States (23.4) and the United Kingdom (11.0) (Royal New Zealand College of General Practitioners, 2000).

There is increasing demand for skilled people and the skill content is changing. There is more emphasis on post school education, as knowledge and information is evolving at an increasing pace. There has been a change in work related injuries as employees are experiencing increasing incidence of societal problems impacting on workplace problems such as violence by the public, less respect and alcohol and drug abuse. 
The continued perception of New Zealand as a healthy, clean and green environment has been challenged in the past decade as the impact of environmental contamination has become more evident, such as fresh and sea water contamination from industry and farming discharge into waterways. Careless burying of asbestos in the 1970s has raised problems now as homes have been built on these previous waste areas and residents now fear the known effect of asbestos. Attempts have been made to address these problems through the Resource Management Act 1991, to set out the management of our environment, including air, water, soil, biodiversity, the coastal environment, noise, subdivision and land use planning in general. Despite this, considerable work is still required to reduce the impact of current problems and to prevent new environmental breaches. Natural and multiple disasters are increasingly reported in national and international media, attributed in part to global warming, although there is some controversy over whether global warming is occurring or whether recent warm years are a statistical fluctuation or consequences of changes in the sun. New diseases are reported as organism resistance has increased, as careless disposal of waste has resulted in trans-species spread of diseases and scientific experimentation has resulted in new drugs linked to genome identification. Changes as a result of global warming are thought to be the cause of: rising sea levels, warmer sub-tropical climates in parts of New Zealand changing the production of food; and ozone hole increases over New Zealand with resultant high 'burn time' and incidence of melanoma. These developments have had an impact on the incidence of drought and increased 
the demand for water and the need for protection from over-exposure to ultraviolet rays.

Economic issues: The current economic indicators reflect that New Zealand generally continues to import more than it exports to a number of the major trading partners. The majority of exports are primary produce or related items and the top three trading partners are the United States, Australia and Japan. The balance of payments of overseas debt has increased as banks have allowed increased borrowing. Foreign investment in New Zealand is gradually increasing, but this is very slow in comparison to countries such as Ireland. New Zealand is increasingly seeking more professionals to meet the demands from the public and private sectors, at a time when many professionals are leaving to meet international demand. More people are leaving than arriving and a significant percentage of these people are professionals recruited to work in other countries. The international shortage of professionals will have a significant impact on the ability of New Zealand to provide services to the population, given the projected ageing workforce and predicted demands of the elderly population projected for 2051 (New Zealand Yearbook, 1999).

Political issues: In New Zealand there has been a change in governance with the introduction of proportional representation and multi-party alliance rather than reliance on the two main parties, either National or Labour in single control. It could be anticipated that this pluralistic approach would continue in the future as groups within society seek to have their voice heard. There is 
general discontent with politicians and their ability to address economic and social issues. Despite the increasing impact of globalisation, the state remains indispensable and it will need to continue to provide more strategic support and stability through economic, social and cultural investments if people are to be encouraged to come to New Zealand and to slow the number leaving for other countries. The role of government has altered over the past decades from providing an extensive social support network to preparing domestic populations for self-sufficiency, global trade and social interactions. The current government is focused on balancing the need for some social support network and mitigating socio-economic disparities in order to enhance cohesion and community, thereby minimising the potential for social differentiation and polarisation (New Zealand Yearbook, 1999)

Economic issues are closely linked to the way people work. While projections about the workplace of the twenty-first century suggest that in the next decade there will be quantum shift in the way in which working and living are perceived, many of the projections are already evident. Already there are knowledge-workers who have expertise in the new and more demanding advanced technology, who work in hyper-automated surroundings and are highly paid for their intellect as part of the knowledge economy (Boyett \& Boyett, 1996).

Five trends are predicted (Boyett \& Boyett, 1998). The first is the 'living company', with companies reconfiguring as small entrepreneurial businesses, with open, adaptive and flat structures. This is already happening in a 
number of industries, as hierarchies become networks of teams with intricate and delicate interconnections and complex flows of information. Smaller organisations are evolving to resemble cellular, organic structures that can form and reform continually, nimbly and skilfully to meet the needs of a constantly changing environment.

Trend two is projected to be a culture that celebrates chaos, with innovation, creativity and challenging of the expected procedures. This new culture challenges the rigidity currently expected of professionals; they are being asked to use their significant knowledge to respond in a flexible manner to the needs of the customer or push the boundaries in what is possible creatively.

The third trend suggests that advances in technology will continue to change what workers do, when, how and where they do it and how they share information. Access to super-fast and affordable computers, optical computers with greater capacity for storage and connections that can transmit considerable information very quickly, will mean that more can be done, in alternative workplaces with connections for consultation. Work in remote multi-centre international call-centres and remote dictaphone transcription and return by email are two examples of what is currently possible.

The fourth trend is cyber-learning where there will be greater emphasis on individualised instruction driven by technology using distance learning and cyber schools to connect learners from all over the world. This is already 
happening with a number of schools working exclusively through internet and teleconferencing. Intelligent applications of new technology will change the content and delivery of information, for example data acquisition and not just reading and analysis. This is already possible in clinical patient information systems that use artificial intelligence to challenge the prescribing by medical staff and to document where they override best practice protocols.

The fifth trend is portfolio living that will allow for choice with a wide range of options of work styles and lifestyles. This is also happening already with young highly skilled professionals working internationally for short periods and moving to new roles for the reward of complex 'packages' (Boyett \& Boyett, 1998).

Other trends predicted and already happening include the growing contingent workforce employed part-time, temporary, contract or in non-traditional work, many as self-employed solo-professionals. Telecommuting has also become a feature of employment with upwards of sixty percent of work done in another location such as home. Upskilling will become an essential and constant feature of the workforce with many people retraining for a new career at least four times in their work lifetime. Self-led teams are a current feature of work settings. The future use of temporary, cross-functional, multidisciplinary teams with globally and ethnically diverse memberships is estimated to be a feature of up to fifty percent of the workforce in the next decade. Everyone will be expected to have strong team skills and be able to 
work effectively in a team from the start, able to undertake a leadership role on the team (United States Government, 1999).

The implications of these trends are that individuals will need to have the skills and knowledge to 'add value' quickly. Specialised generalists will be in high demand using employment agents to find work. Educational attainment is increasing and individuals in high demand will require continuing education to stay ahead. Individuals will need to build and maintain 'liquid' savings as incomes will fluctuate with less full-time employment. Working lives will start earlier and retirement will disappear. The barrier between life and work will disappear, as is increasingly evident. Housing will be wired for commerce, as homes will become home and work sites. For organisations, the core competencies and reason for existence will be essential; traditional management and motivation of employees will not work. Performance goals will become more explicit and measurement will become more sophisticated and objective. Results will count more than activities. Information security will become more of a concern because of telecommuting and contingent employment. In order to assemble teams of the right mix and talent quickly and retain staff, leaders will need to be able to create a shared vision and develop new ways of valuing the talents and knowledge of their workers, linking financial rewards commensurate with the workers contribution (Staiger, Auerbach \& Buerhaus, 2000).

For society the implications are significant. City centres will probably no longer be the centres of commerce as people work from remote sites. This 
could have implications in inner-city building rentals. Many of the buildings could become centres of art, culture, theatre and entertainment, homes for the super-wealthy and also for the homeless. Small towns could also be revived as people return to the 'clean' country and work from home, fostering rural revitalisation. Businesses may not provide social service support and will expect the individual or government to resource these services. Professional linkage and loyalty globally is predicted to have more meaning than nationstate identification.

Technology: The developments in technology in the past two decades have been exceptional. Development and change has occurred in the areas of superconductors, fibre-reinforced composites, high-tech ceramics, computer technology polymers, micromechanics, photovoltaic cells, advanced satellites and microwaves which had already changed industry and healthcare. These new developments have in the past decade set the scene for significant change in health care, communication, information storage, industry and transport.

Genetic engineering with mapping, restructuring and remodelling of the recombinant DNA gene code is now complete to allow for elimination or enhancement of a specific trait and antisense RNA discovered to eliminate defective genes. Advanced biochemistry allows for monoclonal antibodies to diagnose disease, to pinpoint specific genes and purify rare substances, to undertake foetal cell transplants and foetal brain transplants and with 
photoactive drugs allow therapeutic drugs to be placed in a specific location in the body to be activated if exposed to light.

Digital electronics have revolutionised digital imaging, digital interactive television, digital cellular phones, allowing for personal communication networks and anti-noise technology. Optical data storage is possible with advanced compact discs with bar-code readers, optical film. Advanced Video displays with flat-panel displays and high-definition television is a reality. Advanced computers are already in use with electronic notepads, multimedia computers, telecomputers parallel computers and multi-sensory robotics. Distributed computing exists with electronic data interchange, desktop videoconferencing, computer-integrated manufacturing using local or wide area networks.

Artificial intelligence is used in advanced expert systems which can capture the expertise of decision-makers and through rules apply these to problemsolving, through voice recognition software and image processing. Lasers are in wide use including in surgery, in advanced compact disc information storage. Fibre Optics is essential to telecommunications systems, distributed computing systems and endoscopic technology. Micromechanics have developed quantum structures as small as twenty nanometers of only one hundred atoms. Thin-film deposition for diamond thin-film coating ion implantation and ion beam assisted deposition. Molecular designing allows for custom designed materials such as tailor-made enzymes for industry (Burrus, 1993). Nanotechnology has the potential to revolututionise 
healthcare through miniaturisation and remote activation of internally inserted nanotechnology.

New Zealand has taken advantage of many of these developments as part of competition and participation in the global community. Tele-conference facilities have transformed access to information and contact with remote settings. Tele-medicine is increasingly used to access scarce specialist advice and communicate with rural communities. Communication technology allows for shopping from home. There is more two-way communication through teleconferencing, imagine the possibility of no visitors for in-patients but interaction through teleconferencing from their home to the hospital room. Tele monitoring and telemetry already allow monitoring at home which can be used for security and health benefit.

There is improved technology to sustain life with neonatal survival commencing at 20 -weeks gestation. Genetic manipulation is already a feature of current experimentation with food and drugs as well as animal production. Applied genomics with cross-species transplants and transgenic medicines are already a feature of scientific experimentation and production of treatments. Transfer into human treatment is already in practice in some countries. Extension to human reproduction is already a fact. Evidence of the spread of trans-species diseases has raised public concern, with the recent "mad cows disease" scare. Increasing resistance of infections to antibiotics and the return of many communicable infectious diseases because of community reluctance to vaccinate, growing organism resistance and 
increased migration has also raised concern for human safety. Vaccination for certain cancers and chronic diseases is evolving as more is understood about disease causation, genetic pre-disposition and as technology develops early diagnostic techniques. Non- or minimally invasive surgical techniques are increasingly possible, as keyhole procedures have transformed postoperative recovery and allowed for pre-natal correction of diagnosed health problems.

These developments are already in use, with the future planned to advance on these technologies and push the boundaries further. Planning that uses linear forecasting with 'more of the same' is doomed to be obsolescence. Much of what we do will be altered by the drive for efficiency and the natural interest of opinion leaders in innovation and developments.

Driving forces

There are a number of driving forces that are evident from the current situation which will have impact on the future. These are listed in Table 17.

Table 17. Driving forces

Driving Force 1: Scientific and technological innovation

Driving Force 2: Globalisation

Driving Force 3: Development of knowledge and desire for information

Driving Force 4: Economic stability

Driving Force 5: Political and national security. 
Driving Force 1: Scientific and technological innovation refers to the speeding up of all processes, the competitive drive leading for optimisation and rationalisation of existing systems, pushing to more powerful technologies, ephemeralisation or the drive to do progressively more with less.

Driving Force 2: Globalisation is welcomed in some respects, as there is increased exchange of knowledge and cultures. Globalisation, however, has a number of negative aspects, because for example it can be used to maximise the profits of the participating multinational companies at a cost to local sustainable system contexts. Many groups aspire to ensure that globalisation facilitates global progress towards 'quality of life for all', seeking to achieve sustainable human development and sustainable livelihood. Globalisation can have a potentially negative influence because of potential merging of cultures and a uniform commercially driven lifestyle but it can also ensure that there is benefit for all by those that can afford the development costs sharing the developments.

\section{Driving Force 3: Development of knowledge and desire for information} that is almost overwhelming in its speed and development. The quantity of knowledge, the volume of new information available, the pace of change and the vulnerability of information systems to sabotage all have an impact now and on the future. The real benefit of information lies in the selection, not the fullness and in the relevance of the information, not the increasing speed of transmission. The current speed of development and interrelationship of information technology through the world-wide web, 
the use of email and the integration of web-based technologies and other technology increases access to information but also increases vulnerability to destruction of databases and information through 'e-viruses' and human 'hacker' behaviour. Regardless, technology provides the opportunity to enhance communication and problem solving. "Errors in dealing with complex problems can only be avoided through the use of information technology that is system-oriented, through hardware and software that aggregates onto the essential system components and to the recognition of system behaviour" (Vester, 1999, p.5). Solving problems relies on having relevant information to identify trends but to view these in relationship between things, their reciprocal actions, in relation to their role in the system.

Driving Force 4: Economic stability appears to be in conflict with the drive for commercialism and competition that 'trusts the market' and places no controls or protectors in place. There is a move, however, to reduce the debt - burden for third world countries because the World Bank and major governments acknowledge that poverty fosters violence and social instability, spread of disease and threat for the people of the nation itself and those sent to keep the peace.

Driving Force 5: Political and national security. While there are efforts to enhance increased co-operation and global co-ordination, there is suspicion of suggestions to improve global governance in a socially responsible way or increase nationalism or have a global leader step in to fill the political vacuum (Suter, 1999). 
The overriding forces and themes identified from the KPMG Strategic Review of Undergraduate Nursing Education (2001) identify related themes of global and electronic connectedness, consumerism and individualism and scientific development. The authors anticipate less government control, new electronic service delivery models, expectations of fast, immediate service, removal from processional intermediaries, more culturally appropriate services, genetic mapping leading to prevention and early intervention and more outpatient settings for care delivery. The review of the literature and ideas from submissions suggests six trends that will impact on the roles and functions of nursing.

The first trend is technological development, such as phone counselling, technology that will reduce the need for frontline workers, with face-to-face interaction still important but needing a completely new set of skills needed. The second trend is consumer expectations with consumers prepared to pay for the best to get their demands met. This includes the expectations of Maori for Maori-owned and run services, one-stop shop mobile, nurse-led, service delivery models. This also includes issues for Pacific peoples with a younger age group and 'Pacific for Pacific' services. The third trend is demographic and disease projections, with illnesses of old age prominent, urban migration, incidence and prevalence of mental illness rising, Maori health continuing to be an important issue along with re-emergence of infectious diseases, and new diseases in new populations. The fourth trend is location of services, with major nursing in the home-community and isolated communities, highly 
technical hospital care, new roles with a suggestion that nursing could become more like a technician.

The fifth trend is about professional responsibilities that suggest a blurring of professional roles and boundaries, with new disciplines ousting nursing. They predict the number of Maori and Pacific practitioners increasing as well as increased scope and community development roles. Trend six addresses ethical challenges as regards explicit rationing, inability to fund diagnosed problems, pressure on quality because of diminishing budgets. Trend seven relates to policy developments as regards taxation for the collective good, increasing privatisation, intersectoral models, prioritisation and need for a flexible workforce. Trend eight relates to socio-economic influences including unemployment in a knowledge society, the changing role of women and poverty with the gap large and growing.

These drivers and trends are consistent with current indicators in the macroenvironment. There remain a number of uncertainties, however. Questions are asked about whether there is evidence that the current speed and continuous growth in science and technology and its application can and will be sustained. Will society and science cope with the ethical debates, which will arise with the potential for technological advances or curtailed development? Will the scientists and system analysts let the developments stop if society says 'Stop'? Will there be a catastrophe in the use of information technology that will slow developments and the spread of information? Will the negative potential of globalisation be halted by increased nationalism or will a global government assume power? Will 
economic stability be achieved or will market dominance of multi-national companies and control by certain governments continues the inequality? The uncertainties can be described on the following orthogonal axes: one axis is isolation or individual and integration or community (or autonomy versus community), with the opposing axis depicting fragmentation or deterioration and the other extreme coherence or collaborative progress (collaborative and co-operative versus individualised and competitive). These axes will be used in the mapping of the scenarios that follow.

\section{Scenarios: "what if..." and the role for nursing}

In generating "what if" scenarios, information about what is known, surmised or speculated from postulated trends is used to write a story of a possible future situation. Scenarios are tools, created in plural, as markedly diverse carefully constructed set of stories about the future, built around carefully constructed plots, each one modelling a distinct, plausible world that might someday occur. Stories can express multiple perspectives on complex events and scenarios give meaning to these events. In considering the future, at least four scenarios are developed using the predicted driving forces and trends to create a possible future that is worse than, same but backwards, better than and radically different. From these scenarios can be predicted what the role and function of nursing might be.

In Figure 7 two intersecting contrasting positions form the stimulus for the scenarios that follow. The contrasts are isolation compared with integration and fragmentation with coherence. Four scenarios can then be postulated of a 
'worse than', 'same but backwards with greater controls', 'better than' and 'radically different'.

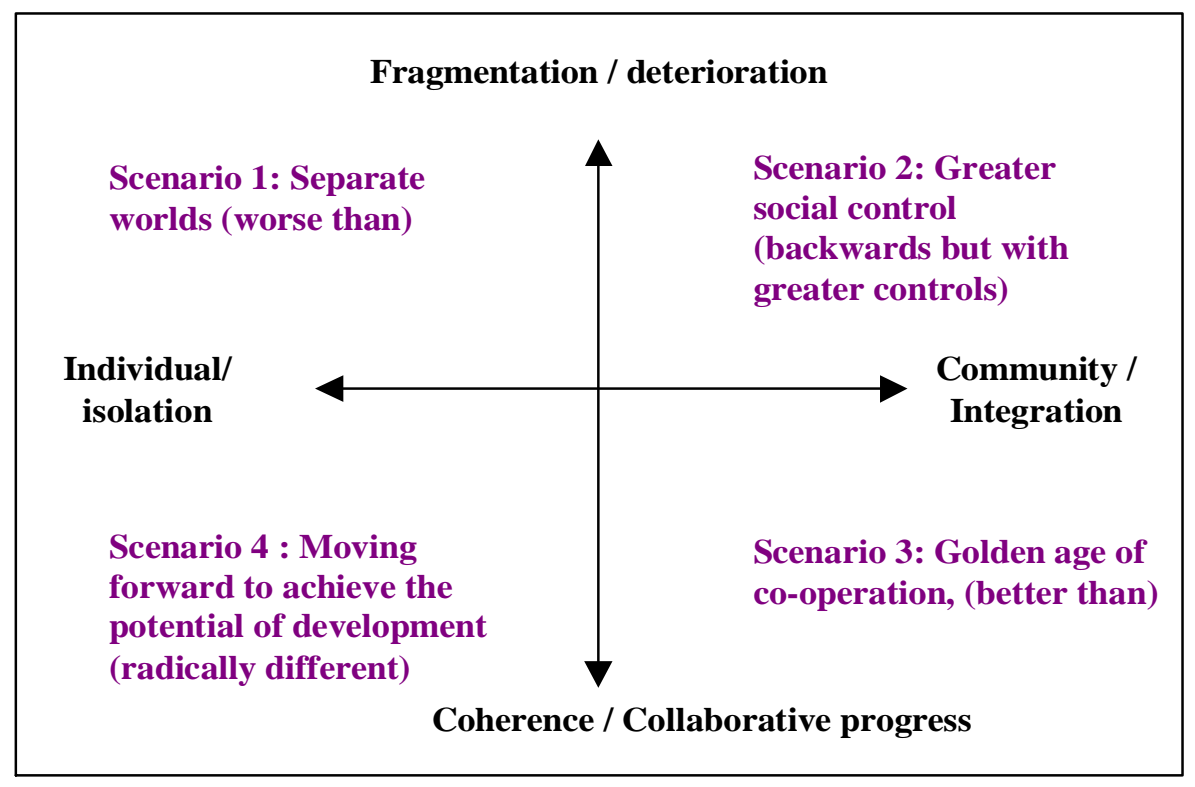

\section{Figure 7. Scenarios based on driving forces and predicted trends}

The following four scenarios are developed from my reading and imagination. They attempt to create a sense of possible future using the 'Contribution Model' framework to create the two dimensional perspective against which the possible actions of nursing can be visualised as the third dimension. I have selected the 'worse than' scenario to start with because it is the easiest to stimulate ideas from based on recent films. The film stories depict a sense of anarchy, social unrest, viral mutations causing major health problems and fear by those feeling vulnerable that assist in visualisation of the potential for this scenario. 


\section{Scenario 1. Separate worlds (worse than now)}

\section{Introduction}

In this scenario the world is much as it is now with local ethnic conflicts, increasing infectious diseases, economic hardship with a greater gap between those in employment in the knowledge industries, those working in the social services and the unemployed. Technology has advanced such that work is less labour intensive and there is individualism and fragmentation, loss of meaning and deterioration of the social fabric. This scenario acknowledges the impact of economic hardship for those in less well-paid roles and those unemployed. There is lack of social control, poor co-operation and expectation of self-sufficiency. The health services are forced to be reactive and can only provide the minimum service given the high demand for services and the finite resources. There is political uncertainty and economic deterioration because the government cannot deliver on the promises or expectations.

\section{Socio-political}

The demand for super-skilled and experienced professionals escalates as people are paid at very high salaries where they have the requisite knowledge. The less skilled workers are employed at reduced salaries in menial service roles. There is higher unemployment for people with less education and these people are resentful of their situation. The highly paid and wealthy become concerned about their safety and live in protected enclaves away from the general populace. The use of the internet is the chief exchange of information which further disadvantages the lesser paid or 
educated people, as they do not have access to all of the technological advantages. Information technology is user-friendly and easier to use. The challenge for most people is the skill required in data analysis. There is potential for manipulation of people who believe what they read, rather than differentiating the good from the not credible information.

In New Zealand the inner cities have been converted into security-living environments for the rich and cultural centres for arts and entertainment. The homeless also live in the shadows of this environment. The suburbs are where many of the lower paid workers live in fear because of attacks by gangs. Many families have moved back to the smaller towns and either work remotely using advanced technology or in smaller self-supporting communities. The elderly in particular are fearful, as the young and highly paid workforce is not interested in supporting social welfare programmes. The elderly are reliant on support workers who are underpaid and under skilled. Many elderly have moved to the smaller towns too where they feel safer. The rest home compounds are over-full and a viewed as 'fair game' for the gangs. Money is in short supply and people live very frugally. While travel is faster and cheaper, many cannot afford to travel overseas.

While many use the cyber-school for education, there continue to be kindergartens and schools for cultural groups who do not have access to the technology and believe that their children are losing Te Reo, their language. It is difficult for people to progress to advanced tertiary learning if they have not had access to the technology from the first. The environment is damaged, 
as money is not invested in replacement of infrastructure and protection of the wildness areas. New Zealand remains cleaner than most other countries, however, because of the smaller population and minimal industry. While the wealthy groups seek New Zealand as a holiday destination because of its apparent cleanliness, they do so in resorts with protection. The indigenous people are less welcoming of visitors and are resentful of the affluence.

\section{Health issues}

Technology has advanced to the point that invasive health care is offered only for major trauma. Those that can afford the advanced non-invasive interventions take advantage of this technology, as there is quicker health recovery and less risk of infection. There is significant progress in health promotion and problem identification using nanotechnology and genetic engineering so that many of the chronic diseases are no longer prevalent for those young enough for this to make a difference. Many of the chronic health problems such as diabetes and renal failure are managed using designer drugs, nanotechnology, and external management of medication release. Higher numbers of people have diabetes and the complications of this disease because of inaction in providing health maintenance programmes by governments in earlier decades. Management of the complications is easier with home based technology such as home dialysis machines and designer treatment methods. For those with existing diseases, namely the elderly population, the diseases of old age are better managed. For those who cannot afford advanced healthcare, there are few options as they rely on a busy

public health system. People fear hospital admission because of multi- 
resistant infections with less ability to treat them with antibiotics. Infectious diseases continue to be a problem, with outbreaks of communicable diseases and difficulty managing the multi-resistant types.

The expected lifespan is reduced for the very poor because of continued poor nutrition, abuse of drugs and alcohol and late presentation to the overburdened hospital system for healthcare. For the wealthy the average lifespan is increased because of access to treatments and resources that maintain health and minimise the incidence and impact of illness. For all groups, the life span extends and the elderly no longer retire but continue to work because of financial need, as well as need for professional knowledge and expertise.

People with mental illnesses struggle to get access to services as funding is scarce and people are expected to access resources for themselves. Depression in the general population is extensive and for those who can pay there are services available and medications to control symptoms. People with chronic health problems and no money to pay for the treatment die because of the lack of money to pay for maintenance treatments. Mental illness is not treated competently because of less skilled workers and overdemand on the few resources able to assist the growing number of people who are depressed and psychotic. Violence escalates and people are fearful about the future. 


\section{Issues for nursing}

Nurses are not well paid as their work is a service industry and the profession is not viewed as a knowledge industry, more as an interpreter of knowledge and a guide through the health system. There is high demand for nurses because of the needs of those people presenting at the over worked public hospitals. There are fewer hospitals because much of the treatment is noninvasive and people do not wish to be admitted for fear of exposure to infection.

The overworked public health system remains to provide services for those not able to afford private, specialist care. There are not enough nurses because of the reduced number entering the schools of nursing and the ageing of the workforce (Hinshaw, 2001). Other health workers such as health practitioner assistants fill this gap and there are many health care assistants. Nurses work more in a supervisory role of a larger number of less skilled workers, working alongside technicians who have in-depth specialised expertise. The work of nursing in in-patient settings is highly technical and specialised. The standard of general care is not high because it is primarily provided by less skilled workers and there is inadequate supervision as the qualified nurse is also provider of care for a complex caseload. The workload of mental health units is extreme with more people seeking assessment than the system can cope with. Although there are better medications available, the difficulty is the lack of services to rehabilitate and supervise patients with chronic health problems. Nursing in the community is dangerous work and those nurses continuing to provide home-based care 
work in pairs to visit the elderly and those managing complex care technology in their homes. There is also increased use of telemedicine contact with the specialist inpatient settings. To be able to cope with clinical requirements, nurses are prepared through a basic undergraduate programme and only a few nurses undertake post-graduate education because of the time and cost involved. Some nurses work as nurse practitioners and they undertake a wider scope of practice, prescribing care and medications, supervising other health workers and undertaking many of the functions of the few medical practitioners.

Questions raised by the case study:

What is the role for nursing in this context and scenario? Is there a role for nursing? This is shown in Table 18 . 
Table 18. Contribution framework outlining the work of nursing in the scenario of the world being worse than now

\section{Expectations of nursing}

\section{Stakeholders}

The patients continue to be the primary stakeholder, representing any person requiring assessment, treatment, teaching and support.

Expectation of the nurse by patients

- Professional manner that is kind, open and helpful

- Supervision of unqualified health workers to make sure that care is safe and appropriate

- Knowledgeable person who is able to interpret information from which to make decisions

- A broker of the services required - facilitating access

- Modified role of nurse, more as specialist assessing need, planning with the patient, providing highly complex care needs, supervising others to provide other aspects of care, with the nurse monitoring progress to achieve the outcomes expected.

\section{Expectations of the nurse by health team members}

- Cross-functional team member able to undertake whatever work is required, a multi-skilled worker

- No delineation of roles, respectful collegial team relationships

- Shared accountability for service provision and care delivery.

- Sharp assessment skills, accurate problem-solving and clinical decision making abilities, therapeutic communication skills, ability to delegate to others, teambuilding skills and commitment to patient advocacy" (American Nurses Association, 1998, p.2).

\section{Expectations of the nurse by health planners and administrators}

- Knowledgeable professional able to work in a cross-functional manner

- Effective supervisor of less skilled workers and the health care environment

- Able to undertake whatever work is required to cope with the demand for services

- Cost-effective service provider able to manage care for specific high cost groups, with emphasis on maintaining them in their homes.

\section{Purpose of the role of nursing}

Provider of health services in cross-functional teams, able to undertake skilled assessment and plan care that others can implement in order to achieve the best outcome possible in the circumstance with the least use of resources.

\section{Knowledge and skills}

- Good scientific knowledge of health and illness, patho-physiology, diagnostic and treatment options

- Good knowledge of mental health presentations and treatment options

- Excellent team work skills 
Knowledge and skills, continued

- Knowledge of how the system works in order to get the resources required

- Political "savvy"

- Information use

- Case management expertise

- People management skills

Anticipated outcomes

- Best clinical outcomes possible given resources available

- Management of large caseload as independent practitioner or nurse specialist

- Efficient use of resources

- Consumer satisfaction

\section{Activities and interventions}

Assessing needs, planning and prescribing care, monitoring the practice of less skilled workers and monitoring patient progress in the areas of -

- Leader of the health care team in the effective management of rapidly changing situations in both the inpatient and community setting. In the absence of medical staff manage initial triage, assessment and stabilisation.

- Uses advanced knowledge of physiology both general and complex, able to manage complex behavioural management situations, and clinical and environmental safety situations.

- The diagnostic and monitoring function including assessment, ordering of diagnostic tests, undertaking of diagnostic procedures e.g. gastroscopy, prescribing of treatments and monitoring of progress

- Administering and monitoring therapeutic interventions and regimens - including initiating medication prescription. Follow-up through nurse-led clinics and community visiting. Monitoring patients with complex acuity needs.

- The helping role, more as supervisor of other workers who provide the support functions, the basic care and treatments. The nurse undertakes more complex activities and provides education and coaching to the patient, family and support worker.

- Monitoring and ensuring the quality of health care practices, including setting the standard, auditing standards and facilitating change of systems and processes to achieve better outcomes.

- The teaching-coaching function, including education of support workers. Primarily involved in teaching of patients and families about self care activities so that they can work in co-operation with the specialist centres using tele-medicine and remote treatment initiation.

- Organisational and work-role competencies, which include advanced team management and co-ordination of service delivery of the health system and use of information management technology.

(Benner, Hooper-Kyriakidis \& Stannard, 1999, (McCloskey \& Bulechek, 1996) 
Achievements. continued

\section{Efficiency}

- Management of large caseload, using advanced knowledge and skills

- Supervision of other workers to achieve minimally safe and cost effective service delivery

- Minimise length of inpatient stay through effective use of self care, referral to case managers and discharge into the community.

\section{Effectiveness}

- Consumer satisfaction with cross-functional team co-ordination and expertise of nurse specialist

- Efficient use of resources using existing staff expertise appropriately

- Achievement of best outcome using best practice knowledge

- Benchmarking of outcome indicators

\section{Responsiveness}

- Adaptability to system requirements in order to co-ordinate and lead teams

- Cross-functional skills to be able to undertake whatever is required

- Adaptability to technology and ability to manage care across the inpatient-community interface using information technology

In this scenario nursing as a profession is an advanced practitioner, undertaking many of the current medical practitioner functions and involved in the assessment, prescribing of care, supervising the actions of less skilled workers and monitoring the progress of the patient in achievement of outcomes. The role is also that of co-ordinator of the cross-functional team, managing the health service system and interface. The contribution of nursing is that of clinical leader, team co-ordinator and health system manager.

In this second scenario there is an attempt to control and manage the society, to enforce use of scientific developments to achieve a healthier population and minimise costs. The role of the nurse is more focused on community care and health promotion as a social agent, ensuring access and addressing need. 


\section{Scenario 2. Greater control (much the same with greater controls)}

\section{Introduction}

Technological acceleration has progressed very quickly with the separation between the knowledge developer employment class and the rest of society. The knowledge developer class include all those working in science and technology pushing the boundaries of what can be done with developments in information technology, advances in health technology and genetic manipulation. The obvious affluence of some and the poverty of the majority have caused tension within societies with resultant high crime and chaos. The governments have assumed greater control because of the escalating violence and unhappiness, directing funds towards work that uses the skills of wider communities and manages the standards of services offered to communities. Organisations are required to participate in civic-responsibility programmes. Lawlessness is not tolerated and is dealt with severely. Nations buy the expertise of the knowledge brokers globally, sharing the cost of the expertise that is required.

\section{Socio-political}

There is a high presence of police to monitor social behaviour and there is low tolerance for poor co-operation. There is a full range of basic social services available to ensure that everyone has their basic needs met, but there is rationing and user pays for anything beyond the basic. Everyone is expected to work even if it is for the minimum wage and supported work programmes are directed towards community support for the elderly and disabled. Everyone is expected to participate in some form of education in order to cope with the 
universal use of information technology that has permeated every aspect of life. The full range of services and consumables are available for people to purchase if they wish.

\section{Health issues}

Basic health care is provided to all through community health centres with whom all patients must register. While some chronic health problems continue, especially in the ageing population, this is less prevalent in the younger generations because of use of technology to screen and treat, especially through interventions prenatally and in childhood. A community support worker is assigned to each home under the supervision of a community nurse practitioner to ensure that all of the needs of each household are identified, that appropriate resources are allocated and individual health status is maintained to minimise use of expensive services where possible. Decisions are made about which citizens are eligible to receive advanced technological assessment and treatments based on the benefit in terms of social contribution.

Decisions are made on what the cost to society will be of non-treatment and having chronic ill health not treated, such as mental illness. There is little need for inpatient care except for trauma and emergencies; there are reduced admissions and shorter length of stay is encouraged because of the major risk of infection. Most other healthcare is provided as a day procedure in smaller hospitals and community centres using non-invasive treatments and pharmaceuticals. If people require ongoing care then this is arranged in their 
home under the supervision of the community nurse practitioner with the community support worker who has a basic education and knowledge of care practices.

There is significant access to flat-screen technology in the home for two-way teleconferencing where people who are unwell can use tele-monitoring for cardiac and other problems, linking to their community nurse practitioner and to specialist medical, nursing and other experts in remote centres. Diabetics are managed using photosensitive medication implants, nanotechnological monitoring and remote treatment initiation. Immunisation is mandatory and major programmes are undertaken to ensure full coverage of the population.

\section{Issues for nursing}

There are a few nurses working in specialist in-patient settings caring for patients with high acuity and specialist needs using highly complex treatment processes. They supervise the overall care provided by technicians and health care assistants. Most other healthcare is done in ambulatory care settings where medical, nursing and other health workers provide appropriate input to improve physical, mental and functional health status. People are visited in their homes for treatment and care through community support workers supervised by well-educated community nurse practitioners. There is extensive use of technology and social control to ensure that population health is maintained. Nurses are skilled workers using advanced assessment, diagnosis, treatment and monitoring skills, providing supervision of less skilled workers. 
Questions raised by the case study:

What is the role for nursing in this context and scenario? Is there a role for nursing? This is shown in Table 19.

Table 19. Contribution framework outlining the work of nursing in the scenario of things are much the same but with greater controls.

\section{Expectations of nursing}

\section{Stakeholders}

The patients remain the primary stakeholders although the government agencies are a major stakeholder as control of the health of the community is a high priority.

\section{Expectations of nurses by patients}

- Assessment of the health needs in the community and a clear plan of what is expected for each individual and household to maintain the optimal health status

- Supervision of the community support worker to ensure that what is offered is of the highest standard

- Planning of their needs and home-based care if interventions are required in an ambulatory setting

- Proactive discharge planning if admitted for inpatient care in order to reestablish self-care.

- Highly specialised in-patient care role.

Expectations of nurses by health team members

- A professional who has contemporary knowledge and uses evidencebased information effectively

- Colleague who shares information about the needs of the community and is accountable for their caseload.

- A professional who uses the expertise of other team member appropriately

- Multi-skilled as part of a cross-functional team

- Highly technically proficient 


\section{Expectations of nursing, continued}

Expectations of nurses by health planners and administrators

- Professional who achieves expectations in a timely and cost-effective manner, meets targets for minimal use of resources

- Professional who is able to provide adequate supervision of less skilled workers in order to meet needs of local community caseload

- Professional able to achieve health through social change, prevent illhealth by improving environmental degradation

Professional able to manage care to prevent exacerbation of chronic disease.

\section{Purpose of the nursing role}

To know the health needs of the community and ensure that appropriate plans, support and range of services is in place to ensure that patients receive the care that they need to regain their health in order to contribute to society and minimise cost of service use.

\section{Knowledge and skills}

- Community and health assessment knowledge and environmental management approaches

- Extensive knowledge of health and illness presentations, pathology, diagnosis, treatment and monitoring issues

- People management skills

- Teamwork skills

- Information use

\section{Anticipated outcomes}

- Community coverage of health services to maintain health

- Recovery from illness as quickly as possible through active planning and support

- Consumer satisfaction

\section{Activities and interventions}

Assessing needs, planning and prescribing care, monitoring the practice of less skilled workers and monitoring patient progress in the areas of -

- Leader of the community-based team in the assessment of need using lifespan, health and social-emotional assessment and planning of care to address need.

- The helping role, more as supervisor of other workers who provide the support functions, the basic care and treatments. The nurse undertakes more complex activities and provides education and coaching to the patient, family and support worker.

- Administering and monitoring therapeutic interventions and regimens - including initiating medication prescription, managing nutrition, managing care in the home using technological support.

- Follow-up through nurse-led clinics and community visiting. Monitoring patients with complex acuity needs. 
Activities and interventions, continued...

- Effective management of rapidly changing situations in both the inpatient and community setting. In the absence of medical staff manage initial triage, assessment and stabilisation. May arrange admission to inpatient centres as required. Uses advanced knowledge of physiology, both general and complex, able to manage complex behavioural management situations, and clinical and environmental safety situations.

- Initiating discharge planning from the community not waiting for inpatient discharge

- Monitoring and ensuring the quality of health care practices, including setting the standard, auditing standards and facilitating change of systems and processes to achieve better outcomes.

- The teaching-coaching function, including education of support workers. Primarily involved in teaching of patients and families about self care activities so that they can work in co-operation with the specialist centres using tele-medicine and remote treatment initiation.

- Organisational and work-role competencies, which includes advanced team management and co-ordination of service delivery of the health system and use of information management technology.

(Benner, Hooper-Kyriakidis \& Stannard, 1999), (McCloskey \& Bulechek, 1996)

\section{Achievements}

Efficiency

- Number of homes visited and supported to recover health

- Early discharge from in-patient care through effective planning and coordination of services

- Reduced need for inpatient care through effective management using community-based services.

\section{Effectiveness}

- Knowledge of the community needs and planning of processes and systems to meet these

- Maintenance of a healthy community with minimal use of resources, managing demand for healthcare. Reduction in illness indicators.

- Utilisation of expertise in the community to offer health promotion and social integration programmes to improve health

\section{Responsiveness}

- Ability to work with all health care needs as a generalist, referring to a range of specialists to ensure that the health of the individual is maintained

- Ability to work across the community-health care interface to retrieve the patient from hospital to their home environment with support processes.

In this scenario there is a role for the nurse as community practitioner interfacing with the inpatient specialty nurse. There is an attempt to promote 
and maintain health through regular contact and targeted health programmes. There is a greater need for nurses with knowledge about communities and healthy living practices.

In this next scenario appropriate use is made of science and technology and there is a greater sense of well-being and community participation. There is a sense of egalitarianism and a regaining of some aspects of the welfare state of the 1930s.

\section{Scenario 3. Golden age of co-operation (better than)}

\section{Introduction}

In this scenario the scientific and technological developments are used to improve the social status. There is global co-operation on key important issues to the benefit of all while retaining national identity and difference. There is strong emphasis on global sharing of resources and attempts are made to clear the debt of certain nations and assist them achieve a similar standard of living. The cost of new developments and payment of the elite knowledge brokers is centralised and then shared by all nations so that there is limited competition. There is comprehensive use of science and electronic technology in all aspects of life.

\section{Socio-political issues}

New Zealand has a small elite but generally has equal share of resources to achieve a good standard of living. Families, the elderly and unemployed have elected to move to small towns to take advantage of the pristine environment, 
cheaper housing and space. Small towns and rural communities are thriving. There is a strong community ethic with local civic pride and governance to ensure all the needs of the community are met. With improved conditions and opportunities for employment there is a more stabilised social structure with senior community leaders connecting with discontents resulting in community cohesion and social sense of meaning. There is less isolation and a stronger focus on quality of life for all. Families at risk are assisted to find reasonable housing, assistance to access food and health care. Maori values of whanau and community have a stronger impact and there is organised input into decision making. There is structured community support for the elderly and preference for them to remain in their homes as part of the community where possible. The government provides targeted funding to support communities achieve self-sustaining but there is increased social sufficiency and less reliance on central government. Everyone has some employment and offers services whether for financial payment or payment in kind. There is strong pride in preventing environmental degradation. There is pride in self-sufficiency in home-grown vegetables and support of the agricultural industry because New Zealand is seen as a major food producer of natural, unprocessed fruit and vegetables. New Zealand is the ecodestination and manages tourism carefully to ensure that the resource is not contaminated. People continue to work into old age in social service roles, able to earn enough to buy the services and technology they desire. 


\section{Health issues}

General health improves because people undertake physical health maintenance, managing their exercise and diet. Disease is prevented by changes in extrinsic factors to moderate genetic risk and because of rational decisions about use of technologies to treat people with chronic diseases. There is compliance with immunisation programmes so that there is less communicable disease infection risk. The average life span increases because people are aware of preventing ill health through education provided for all age groups. As in scenario 2, each community has a community health centre that has a connection to each individual and household in the community and provides direct and active programmes to ensure that health is maintained and ill health is treated as quickly as possible. People generally use ambulatory care services such as radiological procedures, laser, laparoscopic and computer-directed procedures that have replaced most of the surgical interventions.

People receive home-based care in their homes after discharge home from the day procedure centre. If admitted to hospital, the nurse and medical specialists undertake rapid and comprehensive assessments using available technology, initiate treatment in the high-technology assessment and observation areas and contact the nurse practitioner from the community health centre to plan for home-based care as quickly as possible. The emphasis is to not admit for in-patient care unless the person requires highdependency intensive intervention and life-saving support. The risk of infection is a concern and treatment uses an almost sterile environment. 
Families tend to visit the patient using flat-screen technology from home rather than travelling in to the hospital setting. Specialist services are accessed from two main centres and there is extensive use of tele-medicine to receive advice on assessment and care planning from these tertiary and quaternary centres.

The emphasis is on funding generic community health services rather than separation into different specialties and use of the majority of funding in hospital settings. Some health workers are more generic in their function in the team and others work as specialists, working in a shared care arrangement with the nurse as case manager. General practitioners are employees and receive referrals from the nurse to review the care needs of certain patients. The team is perceived as an integrated team with little differentiation of status and role between professionals. Health services are more culturally appropriate and safe because of the education of the workers and the use of cultural support workers.

\section{Issues for nursing}

There is greater emphasis on health promotion and maintenance and this is done through cross-functional team programmes targeted at the needs of the community. The nurse needs to have excellent communication and teamwork skills to work with the various team and provider agencies as there will be less emphasis on professional boundaries and more emphasis on who is the best at providing the care needed at the most reasonable cost. 
The nurse has a role in assessment of needs and tailoring a health management programme for each person or household, referring care to specialists as appropriate so that the anticipated outcomes are achieved. The nurse manages clinics for assessment of ill health and prescribes treatment or makes a referral for specialist review. There is greater collegiality between team members in case review and advice-seeking about appropriate care planning. The nurse supervises the work of the community support worker and annually reviews the health status of the people in the community so that there is clear data to allow for modelling of demand for healthcare. The nurse works in a co-operative manner with all agencies and health providers so that the patient can achieve access for specialist care and return home as quickly as possible. Technology allows for greater communication with people in their homes using telemedicine through flat-screen technology. Wireless technology allows for treatments to be managed remotely such as increasing medications being administered through infusions at home. Nano-technology also allows for people to have complex treatments offered at home. The nurse and specialist mental health professional have more time to provide support and counselling to people with depression or mental illness. They are able to monitor their progress, normalising their involvement with the health system through the generic community centre rather than the separation of mental health services from physical health.

Some nurses work in independent and group practices as independent practitioners having responsibility for the needs of specific groups and providing niche-type services than can be purchased. 
There continues to be a role for nurses in in-patient settings, managing highacuity patient needs, working as nurse specialists and supervising the care of patients. The direct care is provided by technicians and health assistants following detailed care plans and overseen by nurse specialists.

The expectations, actions, achievements and responsiveness of the nurse are similar to scenario 2 and so are not repeated here.

In this final scenario society has made significant changes and health issues are a less important priority. Use of science and technology has advanced to the point that disease and ill health are unusual experiences.

\section{Scenario 4. Moving forward to achieve the potential of development (radically different)}

\section{Introduction}

In this scenario there is the possibility of a vastly different social, economic, political and health care situation. In this situation there is strong emphasis on individualism and the right as a consumer to get the services that are desired. There is also a desire to progress the development of science and technology to its fullest potential, to provide more options for the global community. While there are a group of extremely well paid and wealthy people, generally there is a reasonable standard of living for most people.

Government and private corporations fund technology and science, especially where there is significant benefit and financial reward. 
Technology has developed by 2005 such that there is commercial use of an organic compound for data storage consisting of a crystalline protein that the layering of the 'sludge' can allow for additional layers of storage with the finished product able to hold ten Gbytes of information. By 2010 there are DNA memory devices that allow for data storage of ten petabytes of information in a cube the size of a sugar cube. Science has progressed significantly in the speed of travel so that space travel and colonisation is a reality. Whole generations of people by 2050 live in fully equipped space habitats prepared by nanoscale robots. In 2025 molecular manipulator 'swarms' are introduced that can be remotely controlled to build structures such as eyeballs one atom at a time and are able to perform a million operations per second. By 2030 a nanobot is introduced to neutralise cancer cells. These nanobots can be hypodermically injected into the patient's blood in vast numbers and can be controlled from outside of the patient with acoustic signals. When a cancer cell is found the nanobot releases one molecule of an enzyme that welds a protein to the ribosome killing the entire cell (Platt, 1998). This eliminates the use of pharmaceuticals and radiotherapy for cancer management.

\section{Socio-political}

The government role is to maintain stability and ensure that the general populace has a reasonable standard of living, the poor given vouchers so that they can enjoy the advantages as a consumer. Technology has provided unlimited customised choices and companies have become very customeroriented. Computers do most of the work and new types of work are needed 
to stimulate the intellect of people. Leisure increases and more people are employed as service workers to cater to the increasing demands of consumers. While there are gangs with considerable power, violence is not tolerated and people are treated very harshly if they harm the vulnerable. Elderly live in protected environments with full services. All citizens receive a minimum wage to keep them satisfied and able to participate in the economy. All citizens are provided with a home with the core basics and are expected to care for these items. The core basics include subsidised internet access, flat-screen technology and other essential communication and entertainment technology to enhance communication of social values and minimise the sense of dissatisfaction. Food shopping is available online only. 'Dirty' technologies are outlawed such as use of fossil fuels and chemical that can harm the environment. All nations are required to use clean technology only. Governments contribute to a pool of money to assist poorer nations achieve the goal of total clean technology use.

\section{Health issues}

The government ensures that all citizens have access to the basic health care services, although individuals may pay for whatever else they desire. Health problems are well managed because of genetic manipulation of all foetuses. People also receive access to annual screening and many have nanotechnology implants that identify as soon as possible any problems that may arise. This technology also allows for initiation of treatments using external radio waves. Healthy living is preached to all citizens and the range of products allows for healthy choices. Inpatient facilities are not well 
utilised because there is little reason for admission, with care provided in clinics and home-based services using technology to support home-based care.

\section{Issues for nursing}

There is high consumerism with people wanting choice about how they access and use services. People want to be healthier and as consumers wish to access alternative health maintenance practices. With increased leisure there is increased incidence of sexually transmitted diseases and health education and treatment is required. Some individuals continue to experience anxiety and require counselling. Mental illnesses continue but are better managed with medication and behaviour modification processes. Recreational drugs continue to be used and management of addiction continues to be a challenge.

Questions raised by the case study:

What is the role for nursing in this context and scenario? Is there a role for nursing? This is shown in Table 20. 
Table 20. Contribution framework outlining the work of nursing in the scenario of the world that is radically different

\section{Expectations of nursing}

\section{Stakeholders}

The consumer is the primary stakeholder, is highly knowledgeable because of information on the internet and has expectation of being a partner in decision making.

Expectations of the nurse by patients

- Professional manner of the patient as consumer with expectations that must be met

- Their needs will be identified and met in innovative ways

- A 'total service experience' using the latest technology or innovation

Expectations of the nurse by health team members

Referral for specialist assessment where appropriate.

Expectations of the nurse by health planners and administrators

- Customer satisfaction

- Contemporary knowledge and expertise.

\section{Purpose}

To offer services that will meet the needs of the consumer in the most innovative and evidence-based manner.

\section{Knowledge and skills}

- Specialist clinical knowledge so that the service offered is of the highest standard

- Customer service skills

- Business skills

\section{Anticipated outcomes}

- Customer satisfaction

- Achievement of health goals

\section{Activities and interventions}

- Limited in-patient specialist care co-ordinated by nurses, but with high technical component.

- Community based programmes for health promotion - healthy lifestyle living

- Addiction detoxification and treatment

- Treatment compliance monitoring for treatment of sexually transmitted diseases

- Counselling and support of people with mental illness

- Community-based support programmes for the elderly, disabled to maintain their health. 


\section{Achievements}

- Needs met to the satisfaction of the patient

- Goals achieved

\section{Responsiveness}

- Able to provide the niche range of services required

In this scenario the role of the nurse is focused on health maintenance programmes and fewer nurses are required. The nurse is more of an entrepreneur and provides for niche markets.

\section{Implications of the scenarios for the role for nursing}

In the first three scenarios there is clearly a role for nursing. In the last scenario there is a possibility of the role of nursing being challenged by other providers.

In the first scenario, the community health and social needs are similar same to the current context, with increasing demand for community support that is provided on an 'as needs' basis in an increasingly unsafe community setting. Nurses are needed in higher numbers, along with other health team members, to cope with the increasing demand for inpatient and community-based care. The public with money to pay for access to private services and new technologies will probably use private hospitals and specialist care. The impact of antibiotic resistance will make in-patient settings high-risk places to receive care. The high use of health assistants and inadequate supervision has a detrimental effect on the standard of care and lower the trust of the public in the care provided. There is a high demand for a mental health 
worker to meet the considerable demands for assessment, treatment, support and rehabilitation as well has mental health promotion. While this role could be a nurse, it is possible that this might not be as the blending of health professional roles in mental health services has reduced the nursing identity.

In the second and third scenarios, the nurse could have a number of potential roles. For example, as an inpatient nurse specialist supervising the health team and initiating treatment; or as a community nurse practitioner interfacing with other providers to get the needs of the individual met. A relatively large number of nurses would still be required to provide the level of case management and supervision required. The nurse would need particular skills to work in an interdisciplinary team as a case manager.

In the fourth scenario, there is a question about whether a nurse is needed given the development of technology, emphasis on health maintenance and minimising ill health and disease. While there could be a need for some nurses to provide in-patient care where there is trauma or significant illness, the number of nurses required could be significantly reduced. While there might be a need for a nurse to provide specialist in-patient care or to offer health promotion programmes, there is a possibility that there will be little need for nurses and another health worker may have evolved. If nurses, as entrepreneurs, focus on niche services, they would require very different knowledge and skills. There is a stronger need for the expertise of mental health nurses in this scenario. 
The knowledge and skills required for each scenario are slightly different although there are core knowledge themes such as science and pathophysiology, diagnostic, treatment and monitoring requirements, teamwork, case management, understanding of community needs, resources understanding and people and systems management skills.

The number of nurses required for the various potential futures is an area of particular interest given the current workforce supply crisis. The first scenario requires nurses in similar numbers to the present to cope with the diverse needs of the community in all health settings. This may pose a problem with anticipated decline in supply of full time equivalent nurses that will peak in 2007 and then steadily decline with a 20 percent drop possible by 2020 (Buerhaus, Staiger \& Auerbach, 2000). The nurse as co-ordinator of care, is an active provider of complex care and supervisor of less skilled workers. The nurse and other health team members are inundated with work and work in a multi-functional way in certain circumstances. In this scenario the nurse must have a comprehensive range of advanced technical skills in order to meet the broad demand for health care. This scenario requires more nurses with more knowledge and skill.

In the second two scenarios, the demand for a high number of nurses remains high. There is need for specialist acute inpatient nurses and a number of community nurse practitioners would be needed to provide the level of supervision and home-based treatment and care required. There is requirement for supervision of caregivers and community health workers to 
provide care according to the prescribed plan of care. The role of the nurse requires advanced assessment, planning and monitoring which is consistent with the professional preparation of the contemporary nurse. Science and technology is assumed to have developed cures for many of the present illnesses. There is an imperative to minimise cost and there is therefore increased transfer of care to home-based services. This change in demand because of technological advances and the reduction in need for as many nurses in in-patient settings, coincide with the reduced number of nurses available because of fewer entering the profession and the ageing of the current workforce. The supervision of care and work with a care support worker in community settings will also allow for current nurses to work to an older age which complements the capability of an ageing workforce. Nurses will need to undertake ongoing learning to adjust to changing needs and expectations.

In the final scenario fewer nurses are required: in specialised inpatient settings, some community overview roles and in niche markets roles.

In all four scenarios, the smaller numbers required and the role of the nurse as assessor of health need and supervisor of the health team will mean that nursing will probably be able to meet the predicted expectations in terms of number and function. The older nurse will be able to undertake the clinical and supervisory work if assisted by adequate numbers of caregivers and support workers. While this relies on changes in technology that alter many of the health needs, the role of the nurse remains an essential requirement in 
the provision of health care. The expertise of nursing to work alongside people to identify their needs and help them achieve the goals set is not easily replaced by a new generic health practitioner role. International trends have the potential to integrate nursing and allied health practitioners into one cross-functional practitioner to work under the direction of the unchanged senior medical role, the health practitioner supervising the health practitioner assistant (Cochrane, 2001). The impact these types of initiatives could have a significant impact on the existence of the role of nurse. Having a generic health practitioner role will reduce the ability to recruit into the health workforce as a generic identification does not have the same professional draw. This discussion is a whole new topic.

\section{So what might be the contribution for nursing in the future?}

Scenarios as a collection of futures are intended to establish the boundaries of our uncertainty and the limits to plausible futures (Wilson, 1975, p.3). Do the scenarios outlined above present plausible and meaningful contributions for nursing? While the descriptions suggest the extremes of possibility, there is clearly a place for nursing input into health service delivery across many of the same spectrums or scopes of practice where nurses currently practise. Many of the needs and current procedures will alter with new and yet to be designed technological interventions but skilled comprehensive and holistic assessment will remain a highly sought after skill-set. The need will occur especially when linked to the interpersonal expertise that facilitates joint planning, supported by advanced technical skill that remains focused on the individual's response. 
The contribution of nursing in the future is that of the skilled practitioner, able to lead a team and to manage and co-ordinate health services and systems. The contribution is to restore health as quickly as possible and to work with the individual, family and community to develop plans and programmes to maintain health. Nurses will continue to work as agents of society in disease prevention through initiatives such as communicable disease contact tracing, environmental monitoring and co-ordinating vaccination programmes. The contribution will continue in the areas of health gain.

The nurse as specialist will need to be highly specialised for the high acuity and technically advanced in-patient setting. The nurse as generalist community case manager will need specialist skills in community health assessment and will need to be updated in current treatment technologies in order to provide care in the home. The nurse will need to be a skilled negotiator and facilitator of resources to ensure appropriate access for the individual or family. These nurses will require advanced post-graduate learning to be able to function credibly and safely.

\section{The place of nursing in the future health service}

So how do these scenarios translate into a plan for the future place of nursing in healthcare in New Zealand? While scenarios can be helpful to act as a rehearsal of the future, they need to be taken one step further. Scenarios allow one to identify the warning signs as they unfold 
so that one can avoid surprises, adapt and act effectively. Decisions which have been pre-tested against a range of what fate may offer are more likely to stand the test of time, produce robust and resilient strategies and create distinct competitive advantage. Ultimately, the result of scenario planning is no more of an accurate picture of tomorrow but better thinking and an ongoing strategic conversation about the future (Global Business Network, 2000).

Kane (2001) suggests that some actions will be required regardless of which scenario comes to pass, in order to deal with the likely changes. It is not necessary to develop a complete strategy for each of the scenarios or select the scenario that has the most likelihood or probability of eventuating. "Scenarios as a collection of futures are intended to establish the boundaries of our uncertainty and the limits to plausible futures" (Wilson, 1975, p.3). The approach to move from scenarios to strategy include: a sensitivity-risk assessment based on the resilience or vulnerability in different conditions, so that any modification can be put in place to improve the chances of success. Strategy development and evaluation is another step to look at what strategies we should pursue no matter which scenario materialises, what opportunities arise from the strategies and what threats and risks that can be foreseen or overlooked that will impact on comparative success or failure. The third approach identifies the key elements that will ensure a successful outcome regardless of the eventual scenario that materialises. This approach considers how each element can be addressed to ensure that it will succeed: for example, what focus should education for entry to the profession have for 
the various future scenarios? How might nursing contribution be marketed? or what alliances will be important?

In the four scenarios presented of: 'Separate worlds' (Individual/isolation); Greater social control (fragmentation and disintegration); 'Golden age of cooperation' (community/integration); and 'Moving forward to achieve the potential of development' (Coherence/collaboration), there is a role envisaged for the nurse. The role and functions may require fewer of them. They may work more as a skilled assessor of need, negotiator of agreed care plan, monitor of care provision and supervisor of less skilled workers. There is a risk that with significant technological advancement, there will be less of a role for the nurse in the highly technical in-patient facility, with technicians and others undertaking the narrow, specialised technical tasks.

In the other scenarios, nursing works more in the community, co-ordinating service delivery to maintain health, facilitate health recovery and support the person in their home in old age and in the terminal phase. The risk is that the skilled assessment and facilitation will not be valued and other roles might be put into the co-ordination position, such as social work. Without a clear sense of identity and clearly articulated statement about the purpose and contribution of nursing, the profession is at risk that other roles will replace and eliminate nursing over the next twenty to thirty years. 
So what strategies might be articulated to address this vulnerability?

Regardless of the scenario or composite of scenarios that evolve, it is firstly recommended that the role, function and value of nursing in each setting be clearly and strongly communicated by nurse leaders. This is essential so that there is no doubt of the place and benefit of nursing in these settings and nursing can begin to believe what is said. It is essential also that all nurses be provided with the skills and confidence necessary to articulate their place without reliance on someone else to do this for them. They must be able to measure and demonstrate their contribution confidently and effectively. This development is the responsibility of the nurse leader in each setting and the profession as a whole. The professional leaders must prioritise their efforts and develop clear processes for leadership of nurses, eliminating the focus on other less important activities and political infighting. Providing clear role descriptions, coaching so that information is generated that provides evidence of the contribution and ensuring that education at undergraduate and post graduate level reinforces a clear and strong image of nursing, must be an essential force is a high priority for nurse leaders.

The second recommendation is that the base competencies of the education programme for entry to the profession should alter. There should be more emphasis on: 'nursing as a contributing profession'; excellence in technical and interpersonal expertise to enhance the assessment of health and illness; emphasis on health promotion and health maintenance in communities; negotiation skills to work with individuals and groups to plan care; significant increase in mental health assessment and treatment techniques; and emphasis 
on solid nursing care practices using core principles. This will necessitate a shared relationship with health providers to ensure that the academic-practice link is stronger. There should be emphasis on evaluation of nursing achievements rather than pure research skills that could be learned at post graduate level. All graduates will need to enter 'entry to specialist practice' programmes in their pre-registration and new graduate period, to gain general orientation to clinical practice in order to be safe practitioners in any specific setting. They must have a strong nursing identity and a core knowledge and set of skills, in particular community-health knowledge and a strong mental health base.

The third recommendation is that there should be established a strong suite of post-registration development programmes, starting with clinical speciality programmes from year two post registration that prepare nurses to be clinical leaders. As clinical leaders they will need advanced assessment, care planning and technical skills; to be prepared to influence policy development; to work with different agencies to address the social determinants of health. These programmes need to prepare nurses to take initiative in establishing alliances with primary providers so that community-based care delivery places nursing in a position of influence with individuals and families. These programmes need to prepare nurses who will co-ordinate service delivery, manage teams and the work 'at the front line' as team leaders of the multidisciplinary team. Nurses must learn skills to establish strong working relationships in cooperation with other health providers. 
The final recommendation is that workforce planning be undertaken as an international initiative, so that: the global needs can be understood; preparation of the nurse can be aligned where it is appropriate to local cultural needs; and the numbers prepared as nurses can be consistent with local and internal demands. The current 'raiding' approach has a detrimental impact on local communities. All countries are facing a growing elderly population and a reducing numbers of nurses entering the profession. They all need a nursing workforce to meet the needs of their communities. This is not just a national workforce planning issue, but an international one. It is essential that we learn from the impact of poor planning by some countries, who have increased the unqualified workforce to the point that it affects consumer safety and has now irreversibly damaged nursing workforce morale and retention. It is essential that the decisions about workforce planning and workforce mix are not left to managers, who often have a reactive economic ideological bias. Planning must be led using a joint 'manager-professional' work group who can together debate the issues, focus on the needs and expectations of the consumer and identify future needs.

These recommendations are not made as a defensive strategy to protect the place of nursing or foster separatist perspectives but to ensure that nursing is present as a viable option in health care delivery in the future. Consumers must be able to choose to receive care from nursing as well as other disciplines. Nursing has something unique to contribute into the future. To be present in the future will require some active work from professional leaders. We cannot rely on past and present understandings of the value of nursing to 
the health of New Zealand but need to create a new understanding of the role and contribution of nursing to the health of the nation.

We also cannot isolate professional development from the broader societal and social policy issues in the workplace, education, economics and politics. Key transitions in society, health care, the workplace and nursing affecting professional development include societies' value-for-money orientation and government messages for citizens to assume more personal responsibility; regulatory changes to assure continuing competence; changeovers to personal work contracts; move to community-based services; and workforce imbalances (Oulton, 1997, p. 47-48).

These recommendations are generic and conservative. They do not take a strong position on what the future state might be. In fact the four scenarios developed in this chapter do not push 'radically different' or 'worse than' to the extreme of what is potentially possible. In other situations when undertaking this futures-thinking exercise with groups of nurses in strategic planning processes, the ideas of participants have extended beyond the ideas proposed above. The exercise makes some people very uncomfortable and other are excited by the potential of forecasting. All participants have, however, experienced the value of thinking 'outside the square' and then being able to plan processes that can influence and maybe alter the direction of their projected future scenario. They have all observed the difference in their altered perspective from the linear, 'extension-of-now' approach to considering a range of different scenarios. 
Having futures-thinking competency is important for professionals as the drivers of political change become evident in moves to restructure the ways in which health care professionals are regulated, to address professional numbers to meet the public needs yet not create oversupply to produce excess costs or waste of scarce resources. Professional schools are being challenged to realign training and education to be more consistent with the changing needs of the care delivery system and to foster interdisciplinary competence in all health professionals (Pew Commission, 1998). Planning for a future that is 'more of now' without considering the alternative futures could result in a professional response that not in line with trends evident in the political agenda. The profession has the potential to be mis-aligned and reactive in a future environment when proactive thinking and entrepreneurship is the successful mindset.

This chapter has considered the role and contribution of nursing into the future, using scenario planning to consider a variety of possible futures within which nursing might make a contribution. The 'Contribution Model' has been used to communicate what the work of nursing could be in a range of possible futures. A range of recommended strategic actions have been suggested that will strengthen the resolve of nursing to be active, visible and present in the future.

In the next chapter, the ideas developed in the previous three chapters are drawn together to consider: whether the 'Contribution Model' has been provided a way to articulate contribution in a user friendly way; whether the 
examples presented in the thesis show that nursing has made a contribution of nursing to the health of New Zealand; and whether this thesis has contributed to the body of knowledge of the profession. 


\section{Chapter Eight}

\section{The measure of contribution}

This thesis started as an idea, a search for a way: to communicate the value and contribution of nursing input into health service delivery; to find a way to communicate with decision-makers about the value of nursing contribution; to empower nurses to believe that they have made and will continue to make a difference; and to show how the conceptual model can be used in many contexts. This chapter brings together discussion from the previous chapters about the contribution of the concept 'contribution'; the contribution of the model in communicating the value of nursing to health gain; the contribution of the conceptual model to nursing knowledge and the professions ability to communicate its value.

\section{The contribution of the concept 'contribution'}

As discussed in Chapter 2, the concept of 'contribution' and its application to nursing arose from common usage and the search by the profession for a way to communicate the difference it makes to health gain. When the concept was examined, its relevance to nursing became clearer, especially as the word and its potential meanings reflected many the attributes of the profession. The contribution of the concept 'contribution' to nursing is considered from the perspective of relevance and language.

Contribution as a concept, has been shown to be relevant to the discipline of nursing. Analysis of the word and its meaning has emphasised purposeful 
action to achieve a common purpose, namely 'health for all'. The interpretations of the concept were applied to nursing practice across a variety of contexts, profiling the significant contribution the profession has made to individuals and groups to enable them to participate fully, in the functioning of their life potential. The concept of contribution implies a team effort, a shared vision and purposeful work to achieve a common purpose. This is where the concept is of most relevance to nursing as it reflects the way in which nursing works, collaboratively with communities and other disciplines to co-ordinate care and services to meet identified need. As the previous chapters have shown, the action of contributing requires that there is an assessment of need, that priorities have been agreed, that assessment has been made of what is required to be done and of who is best to do the work. It implies that action is undertaken to meet the expectations and to achieve the expected outcomes. The concept therefore is relevant to nursing and is more than just a descriptor but provides a way for nursing to communicate.

The concept is also of value to nursing because it provides a language and a way of describing the contribution of nursing to be more than a myriad of actions over a century of organised professional involvement. The framework can be applied to show practice in diverse settings and roles and how the achieve the expectations of society. The concept, as it has evolved into a conceptual model, provides a way to link nursing action to the expectations of society and the achievements of the discipline. The model encourages nursing to state the acknowledged expectations, using structured 
language and requires a statement of anticipated outcome, rather than emphasis on tasks, actions and implementation.

\section{The contribution of the model to communicating the value of nursing to}

\section{health gain.}

While nursing is acknowledged as being involved in all aspects of health service delivery, there has been less work done to communicate specific contribution to health gain. Instead, nursing action is described generally as providing care at all levels - primary, secondary and tertiary, they work in all settings, are the largest category of health workers in most countries and provide care for the vulnerable and disadvantaged across the continuum of choice and opportunity. Nurses are in direct contact with the population at large - all strata and in all conditions - for extended periods of time. Nurses possess and control large amounts of information, are frequently the main connection between the individual and family and other health professionals and community agencies; nurses have competence, knowledge and specialised skills, have access to communication channels. Nurses acquire influence by demonstrating their capacity to act - by demonstrating their capacity to change policies - by demonstrating their capacity to establish new programmes, by taking action (Labelle, 1985).

The contribution of the model to communicating the value of nursing to health gain is found in the requirement to link the expectations of the various stakeholders with the actions taken to deliver the anticipated outcome. Too 
often there is a focus on action and interventions isolated from social expectation or reference to outcome and health gain. When asked to explain the contribution of nursing to outcome, there is difficulty in providing evidence, measurable outcomes, and economic benefit. Using the model requires the user to think about outcomes before initiating action and to consider what approach might achieve the outcomes expected.

The model requires that nursing does more than provide the human face of caring for individuals and groups of all ages. The model requires that nursing articulate the changes achieved in the individual or groups with whom the nurse works to achieve a healthy lifestyle. The nurse is required to achieve clarity in describing goals and anticipated outcomes when nursing facilitates restoration of health, rehabilitation, coaching for self-care or palliative care for those dying. The nurse is encouraged to document cost savings where their work reduces the need for admission, or achieves early discharge or prevent complications through early skilled intervention.

The contribution model links to accepted classification systems for description of interventions, rather than reliance on personal descriptions of tasks. The intervention classifications provide a way of communicating the skilled actions of the nurse using language that is recognised by other disciplines and links to acknowledged outcomes. Using this language, nursing is able to communicate the direct benefit of nursing practice to anticipated outcomes. Nursing has previously been less visible in the complementary role in the interdisciplinary team. Use of this strong and 
purposeful language and linking action to clear outcomes, positions the profession to be acknowledged explicitly for the difference it makes to health gain.

The model provides a way for nursing to quantify the benefit of nursing work in care co-ordination and case management, working with individuals or communities 'at risk', with complex needs and co-ordinating multiple service inputs. Nursing involvement in co-ordination of health promotion programmes can be communicated as more than just 'a pair hands' and can show achievement in the reduction of risk and minimisation of harm.

Nursing actions can be shown to have achieved improved functional status and enhanced self-care and independence through the outcomes of teaching and counselling. Outcomes can be demonstrated in symptom control; in stabilisation and improvement of the physical, functional, physiological, psychological and cognitive status of individuals; in improving family caregiver coping through education, coaching, counselling and encouragement. The financial and economic parameters defining contribution has not been developed in this thesis but will be explored in post-doctoral research. This is an important next phase given the emphasis on business models in health care management today. Consideration needs to be given to use of the model as a business model to assist nurse leaders balance the dimensions of cost and quality in nursing practice. 


\section{The contribution of the conceptual model to nursing professional}

knowledge

The contribution of the model to nursing professional knowledge can be explained by the value of having a model to assist in extracting from practice a description of what nursing does and achieves. This will be important to assist nurse educators in their work with nurses at undergraduate level where nurses are learning to differentiate what nursing is and how actions link to expectations and achievements. In post-graduate education nurses are learning to be professional leaders and to establish specialist practices. They need to be able to describe the scope of their practice, negotiate expectations and demonstrate expectations. The 'Contribution model' provides a way for nurses to make these things explicit, to draw together their nursing knowledge to show what they do to meet expectations and the difference they make to health gain.

In reviewing the nursing literature, no other work of this type was evident. Some of the ideas are similar to the work of Evelyn Adam (Harbour et al, 1998, p. 516-528), whom I have recently read. Regardless, one of the requirements of academic work is that the thesis should contribute original thought, it should be unique and original. The work should suggest new ways of thinking and in the communication of the work it should have universal application, able integrate with ideas from other research. The work should apply to a broad context and have a global scope. The work should provide a constructive perspective and be a resource for the profession. The 
'Contribution model' as presented throughout this thesis achieves all of these criteria.

This thesis was developed as a 'thought-experiment' to answer a question about whether there was a way to assist the profession to articulate its contribution to the health of the nation. This work has explored an issue that is of international interest and concern, namely a way to articulate contribution and show the value of professional action. The need to articulate the function and value of nursing practice has been viewed by nurse leaders as one of the most important issues facing the profession as it "struggles to retain its identity in a health care system being restructured for greater efficiency" (Johnson \& Maas, 1997, p.6). The challenge for the profession has been to become more visible as it has sought recognition of the important and pervasive role nursing has in the care component of health care and its effect on health outcomes. I believe that this thesis contributes to this agenda and provides a tool that can be useful for nurses in a wide range of settings and roles.

The 'Contribution Model' can be used by the profession in multiple ways. The model can be immediately useful, as it is relatively simple to use; it provides a clear way of thinking through the link between expectation, action and achievement. The model can be applied to everyday practice and requires little interpretation. For the busy nurse with limited infra-structural or leadership support this is essential. The tool can also be used for orientation of new practitioners who are grappling with their role, for 
coaching individuals in roles where the role is large and complex and the expectations are diverse and overwhelming. The model can be used for strategic thinking and planning and for communicating with planners and administrators.

The thesis contributes original thought to the discipline. It has been sourced from personal deliberation but has also relied on contemporary literature and philosophical processes of analysis and reflection. The thesis is situated in the context of health and professional mandate. The methodology is nontraditional that has forced new insights. The work has developed ideas from an initial concept to generation of a tool or framework that can be applied using case study and scenario planning. While this has been a 'thought experiment', the work has sought to demonstrate validity through analysis, reflection and interpretation of ideas consistent with philosophical expectations. The work has generated ideas and linkages between a range of concepts such as social mandate and professional contribution that has not been widely discussed in contemporary literature. The thesis has firmly established nursing as essential to societal achievement of health strategies. The thesis has demonstrated the value of a philosophical theoretical approach to address an idea. This method does not use traditional research methodologies but has provided an alternative approach for researchers considering an idea.

The thesis has discussed the place and contribution of nursing using a contextualised framework, communicating contribution over time: in history, 
in diverse contemporary settings and the future. This approach is important, as the profession is linked closely with its past yet it is challenged to understand its future contribution. Regenerating pride in past contribution opens the professional to the possibility of pride in contemporary practice. This work has brought historical information from the literature that has not been published in this way before and profiles the significance of early pioneer nurses contribution to health gain. While there is some understanding of the diversity of contemporary practice, there is little written about the complexity of the work undertaken in specialist settings work. The flexibility of the 'Contribution Model' framework promotes structured thinking and presentation of facts.

The thesis re-orientates the profession to seek understanding of the various stakeholder expectations and to clarify the purpose of their work before focusing on the actions to be undertaken. It is essential that the work of nursing be grounded in this understanding of the expectations to prevent the profession drifting away from its connection with those whom the profession seeks to serve. The model focuses on outcomes as a way of evaluating achievements. This is not an area of strength for individual practitioners and will encourage evaluation and clarity of communication.

The 'Contribution Model' is a start, a small beginning that can be added to incrementally through further application of the model and development of aspects such as the economic modelling. The 'Contribution Model' is one tool of creativity to help the profession confidently communicate its value in 
the present and into the future. Futurists suggest that individuals and professions will flourish where they learn to operate in the changing global environment and apply their experiences, seeing connections and finding opportunity where others see chaos. The 'Contribution Model' has been developed to provide a simple guide, to help individuals and groups find the connections and linkages, to take a lead rather than wait for a leader, someone to do it for them. The work of this thesis is still evolving, a "little great idea" that can continue to be worked on by other people (Senge, Kleiner, Roberts, Ross, Roth \& Smith, 1999).

The thesis and the ideas contained within this work contribute to nursing knowledge and provide the profession with a model and framework that can be applied confidently. There are a number of other research questions that arise from this thesis. The model as applied to business planning needs to be developed, including the metrics of finance and clinical outcomes. Given the impact of restructuring of hospitals and health systems there is an opportunity to use the model to discuss the contributions of nurse leaders and to examine team contributions in healthcare. How this model could be developed for use across the educational system of nursing will require some consideration, especially if the concepts of communicating contribution are to be integrated into every day practice language. Integration into education and practice use will strengthen the model. 


\section{Challenging the profession to take action}

This thesis challenges the profession of nursing to take the initiative to communicate its value, using a model that assists nurses state positively that nursing contributes. Unless nursing takes the initiative there is the potential for nursing to be replaced by other health workers because the caring and comfort connection of nursing is not valued sufficiently. The role and function of nursing cannot be experimented with and is not easily replaced by multifunctional workers. Once dismantled the professional focus is not easily regained. Nursing is the unique blending of professional socialisation and commitment, scientific knowledge, special but practical nursing expertise and an understanding of the human experience that places nursing alongside the lives of everyday New Zealanders. This blend has evolved over the past century but remains fragile and vulnerable to political and management trends. If the role and function of nursing is tweaked and dismantled for pragmatic short-sighted reasons, the results will not be retrievable. The profession cannot wait expectantly for acknowledgement or reasonable thinking by decision-makers, but must take responsibility for creating the shared understanding of the value of nursing and ensuring that it remains connected to the expectations and needs of the community it serves.

Being explicit about nursing contribution is important. Treasuring something places a value on it; it keeps the item at the forefront of ones attention and results in care and attention towards the item. In order to treasure something one has to acknowledge it, to apply criteria to describe what is important about it and to put processes in place to protect it from harm. Works of art 
are treasured and protected. Why should the art and science of nursing practice be any less valued?

In the context of nursing as a profession, the concept of treasuring is critical. Unless there is an explicit naming of the value that is placed on nursing contribution and acknowledgement of the criteria that explains why it is valued, there is a danger that the profession could gradually lose focus, lose hope and in time no longer exist. There is a real possibility that the work that nursing does could be replaced by a new generic health worker who undertakes some functions currently undertaken by nursing. What is so intrinsically important and implicitly valued about nursing could be lost in the evolution.

What this thesis has shown is that the actions and achievements of the profession have been considerable and for many communities respected, appreciated and valued. The valuing of the achievement has not always been explicitly documented. Nursing has been so busy 'doing' because that was what was needed that the documentation of the contribution of nursing over decades of involvement is not published.

2001 marks the 100-year anniversary of the registration of nursing as a profession in New Zealand. This was the first country in the world to acknowledge the need to value and protect nursing contribution by passing a separate statute. Nursing was identified as a valued function in society in order to achieve 'health for all' and to build a strong productive community. From the late 1800 s nurses were seen as essential to the progress of the new 
nation. Their ability to organise, manage, co-ordinate, follow direction, do whatever was required however menial, and from the early 1900s to go anywhere, to fit in with different communities, to get alongside individuals and groups to work out the best way to achieve health gain: these were valued actions. For the early local communities, 'their nurse' was a valued asset, a professional they would pay to retain, provide and protect. There was an expectation that the nurse in the local community, whether in active employment or away from formal practice, would go where asked, would do whatever was necessary, would lead and co-ordinate in emergencies, would advocate for the needs of the community with the various agencies and would be accessible, approachable, responsive to all and everything. Nursing involved twenty-four hours a day commitment in many communities, extending past retirement, a social commitment that went beyond mere employment.

Despite decades of action, professional research and debate, the profession continues to lack confidence in many ways to express their purpose. Through the writing of nurses in New Zealand and the deliberations of this thesis, the purpose of nursing becomes clearer. Nursing is a profession that is essential to the co-ordination of accessible and acceptable health services and the provision of safe comprehensive health care. Nursing works with individuals, communities and populations to achieve health gain, to maintain and restore health and provide support through life until after death. Nurses work with people to assess their needs, co-operatively plan the goals to be achieved and work responsively with them to achieve the anticipated outcomes. I believe 
these two descriptions encapsulate the main ideas developed through thesis. They describe the place of nursing in health promotion, maintenance, restoration and support. They describe the expectations of nursing, the way in which nurses' work and what is achieved. These descriptions establish nurses as connected to the aspirations of their stakeholders, responsive, working in co-operation, flexible and able to work across a broad spectrum of need, willing to follow with the individual, group or community to achieve their goals.

Within this broad spectrum of health care, the phenomena of particular concern to nurses are individual, family, and group responses to actual or potential health problems. These human responses range broadly from health restoring reactions to an individual response to episodes of illness to the development of policy in promoting the long-term health of a population. The purpose of nursing is to work with these phenomena and responses in order to assist the person regain self-care and independence.

\section{What has been the nursing contribution to the health of New Zealand?}

The contribution of nursing has been firstly in the domains of management and co-ordination of the services offered across the country. From the disorganisation of early hospitals and health services to the co-ordination of organised services of the late 1800 s, nursing practitioners have managed the environment and clinical systems, have deployed resources, set and applied accepted standards that have supported the functions of the rest of the health care team. The second contribution of nursing has been in the improved 
standards of personal care provided to individuals requiring support to regain health. This has been achieved through psychological support, basic physical comfort care and application of consistent techniques and practices where the person is not able to do what they would normally do for themselves. Family and carer support and advocacy have remained an important contribution, yet it is the least publicly recognised component of the role of the nurse. Nurses have adapted to new technology and practices to provide the acute interventions in the safest way. The contribution of nursing has been to work with individuals and communities at risk, regardless of their personal risk as an individual practitioner. The third domain of contribution of nursing has been in working in programmes to promote healthy living. Nurses have been involved in initiatives to prevent disease such as immunisation; in actions to prevent spread of disease including communicable and infectious diseases; in actions to address the social determinants of health through work with children and families at risk. Nursing has worked in programmes to promote safe work environments, to assist people return to work. Nursing has worked to minimise social isolation and facilitate integration, to enhance social participation.

The contribution can be measured as outputs and outcomes through gathered indicators, evaluations and research. A range of measures of the impact of nursing contribution can be generated from population-based initiatives. Initiatives such as: populations immunised, reduction in the incidence of infection, goal attainment. Other measures include return to full functioning after an acute illness through skilled rehabilitation, improved self-care skills, 
increased adaptive behaviour, reduction in re-admission or frequency of use of services through skilled case management. While league tables of measurement relating to nursing contribution are not easily accessible, the ability to measure is possible and achievable with time, effort and coaching.

The contribution of nursing to health gain has been both visible and invisible. The contribution has been largely unmeasured and has been mainly personal as well as part of anonymous collective action. The contribution has been responsive to changing needs and demands, always present and willing to move into more diverse practice roles. It has been the closest thing to partnership, by working with the same frame of reference as the patient and their family and working to achieve mutually agreed goals to restore self-care and independence. Nursing contribution has been to work where few wished to work, to do what few wish to do, to walk alongside individuals and families forced to confront cultural taboos or major lifestyle changes. The contribution has been the skilful presence of the professional to provide support and coaching. Nursing has provided consistent service over time through practitioner actions that are bound by a social commitment that is not necessarily achieved without the socialisation, common value base, and professional foundation of knowledge and skills to create a sense of professional unity of purpose and profession-hood.

The place and achievements of nursing are valued by many but their import is often unrecognised. Being all things to all people, going wherever asked and doing whatever was asked has been the professions response to identified 
need. 'Fatal availability' and the skill in making self-care achievable, has in some respects, worked against the profession.

Decision-makers in many leading western countries view the comprehensiveness of the nursing function as desirable but generally support a more generic, 'multi-skilled' health practitioner as the worker they want and need for the future health service (NHS, 2000). In some models of health workforce, practitioners are supported by unqualified health assistants and medical contribution remains unchanged from the current functions. The health planners fail to understand that professional commitment develops from identification with a common purpose and way of perceiving the value of the work. Without a professional linkage there is potential for loss of a sense of purpose, difficulty in recruiting people into a health career and a worsening of the workforce crisis. Attention to these possibilities requires attention and cannot be left to chance.

The community know what they value and why, although they are not canvassed in this debate. They receive whatever is provided for them, regardless of their need for social focal practices representing stability and consistency. It is critical therefore to articulate the contribution of nursing so that this is understood and valued by the community. The communities need to be informed about the predicted changes in order to be able to debate with decision-makers about what they want and need. It is the responsibility of nursing to explain what has been achieved in health gain and social 
development through nursing involvement so that the community can understand and decide about the impact should the workforce change.

The contribution of nursing to health is evident if one knows what to look for. The contribution is significant if one places a value on what has been achieved. We can make explicit this contribution if we use a model, common language and economic measures. Nursing is essential to the strategic health and social goals of the nation; it is the 'glue' that keeps the health service together and the 'lubricant' that keeps the parts interacting. While nursing is a sub-system of the main health system, it is also the central input and process component of the health sub-system in society. The power inherent in the position as a central force is under-developed. The profession has a responsibility to take advantage of this position and ensure that the purpose, function and contribution of nursing is acknowledged by the community and decision-makers, using whatever framework is helpful.

The profession could wait for external acknowledgement but this could be a while in coming. Leadership is required to rally the powerful emotional response that nurses feel about their professional work. Its time for action with our communities, that will focus the mind and engender a sense of respectful listening. A 'call' to the profession provides a reminder and a connection to the core of what draws nurses to nursing and the community to value the practice of nursing. It is time for the call to go out.

For decades nursing has remained complacent about its place in health service provision, confident that the value of the work done was understood, 
that the 'what' and 'how' of nursing was appropriate and directed by the profession. In the past decades, this has been challenged as many nursing positions have been replaced by unqualified workers, as other health workers have entered the service settings to do part of the work previously done by nurses. The profession has largely waited for others to protect and defend the place of nursing, unable to provide clear evidence of the value of the professional input, unclear about the focus of the work of nursing. This passive acceptance and waiting for leadership has undermined the confidence of the profession and the community in the professions' contribution. The 'Contribution Model' proposed in this thesis has provided one step towards helping the profession help itself.

We cannot assume that managers and health planners understand the work of nursing and so it is up to us to find a way to communicate with them and our wider community. If we don't know what nursing does and what difference the professional contribution makes, then we have to do something about making this clearer, using language and data that the various stakeholders understand and ensuring that it is communicated by professionals confident in what they say.

The future of nursing as a profession - in contrast to nursing as an activity - is dependent on the way in which nurses are able to address the needs for care of any society within a professional framework. Neither 'caring needs' nor 'caring practices' can of themselves define nursing. It is for nurses to identify a professional frame of care, which will enable needs to be met through the practice of the nurse. And this 
may require a total rethink on the part of nurses about their role and practice (Parfitt, 1995, p.56).

The challenge is clear for the profession. Take the initiative in communication within the profession and with the community we serve. While it might require a rethink of the role and practice of nurses, this may not need to be a revolution, more a 'spring-clean' and 're-branding exercise'. The 'what' that nursing contributes continues to be relevant and needed into the future. Aspects of the 'how' of nursing could be reviewed in light of advances in technology, evidence and changing social expectations. Adapting to the future will require some strategic thinking, negotiation and reframing.

Nursing has a unique function in health service provision and co-ordination. While the professional nursing practices are essential to the provision of safe and effective health outcomes, it is the co-ordination of care activities that lead to the smooth running of health services and achievement of the goals set by and with the patient and their family. Nursing is a bonding discipline "pulling things together, teaching other health workers about the importance of caring, moral commitment, standing up for patients, and what true dignity is" (Seedhouse, 2000, p. 66-68). If this is the case then nursing must be there into the future for the communities we serve.

My dream is that nursing will develop a strong self-image, will be confident in its expression of its value and that practitioners will be clear about their 
purpose, their connection with the expectations of their community and will act always to achieve these expectations. My dream is that every New Zealander and every community will continue to have access to the power of nursing expertise, regardless of whatever future scenario emerges. Nursing practised confidently and well is essential to the health and well being of every community.

It is therefore essential that nursing is present into the future as a strong and viable profession, confident of its place and contribution. The professional contribution of nursing cannot be eroded, substituted or replaced. The health of the nation is too important for the central thread to be tampered with. The value of nursing is in its entirety, in its comprehensiveness, in its integration of caring, technical competence and its practicality. Nursing by its very actions depicts 'contribution as action with purpose'. Nursing is the skilled action used to meet health needs, is undertaken in co-operation with others for a common purpose of 'health for all'. The profession has never sought to walk alone but to work in co-operation to meet the health strategies essential to the growth and development of our nation. We need to be there to be able to contribute. Making sure that this happens is not the responsibility of one person or one group but the action of the whole profession working with our communities to decide what is best for our shared future.

Ma pango, ma whero, ka oti te mahi.

The objective is achieved with the co-operation of all. 


\section{Appendix 1. Nursing Competencies}

Nurses are essential to the safe management of the patient care environment and co-ordination of appropriate patient care.

The nurse is essential for

- The effective management of rapidly changing situations

- Setting up the environment for the management of a crisis

- Sequencing and managing the logistics of rapid multiple therapies in response to a crisis

- Organising the team and orchestrating their actions during a crisis

- Exhibiting experiential leadership in managing a patient when a physician is present

- Taking necessary medical action to manage a crisis when medical staff is absent

- Skilled performance in extreme life-threatening emergencies

- Rapid grasp of a problem, clinical reasoning in transitions

- Contingency management and rapid matching of demands and resources in emergency situations

- Identifying and managing a patient crisis until physician assistance is available

- Recognising changing clinical relevance, clinical grasp and responsebased practice; seeing the unexpected

- The diagnostic and monitoring function

- Clinical knowledge in specific patient populations

- Detection and documentation of significant changes in a patient's condition

- Providing early warning signal, anticipating breakdown and deterioration prior to explicit diagnostic signs; diagnosing and managing emergent and/or life-threatening situations; diagnosing, monitoring, titrating and providing instantaneous interventions to maintain vital functions and physiologic stability in unstable patients

- Clinical forethought by anticipating and preventing potential problems

- Anticipating patient needs: understanding the particular demands and experiences of illness, clinical forethought about specific diagnoses and injuries,; anticipating crises, risks and vulnerabilities for particular patients

- Administering and monitoring therapeutic interventions and regimens

- Co-ordinating and managing multiple instantaneous therapies

- Starting and maintaining IV therapy with minimal risk and complications

- Administering medications accurately and safely 


\section{Therapeutic interventions and regimens, continued}

- Combating hazards of immobility

- Creating a wound healing strategy that fosters comfort and appropriate drainage

- Limiting the impact of painful procedures

- Bridging the gaps in patient care

\section{Monitoring and ensuring the quality of health care practices}

- Assessing and organising a reasonable level of care

- Recognising and communicating the transition from curative to palliative care, planning and implementing attentive palliative care

- Providing a back-up system to ensure safe medical and nursing care

- Assessing what can be safely omitted or added to medical orders

- Getting appropriate and timely responses from medical staff

- Communicating missed timetables and unexpected changes in clinical trajectories

- Frontline quality improvement, monitoring and risk management

- Supporting patients and families facing death

- Teambuilding especially in the context of pressure; building and preserving collaborative relationships

- Minimising healthcare system failures in destabilised work environments

\section{The teaching-coaching function}

- Timing - capturing a patients readiness to learn

- Assisting patients integrate the implications of illness and recovery into their lifestyles

- Eliciting and understanding the patient's interpretation of their illness

- Providing an interpretation of the patient's condition and giving a rationale for procedures

- Coaching

- Teaching and supervising less experience nurses and health assistants, facilitating the clinical development of others; coaching others in interpreting, forecasting and responding to patient transitions

\section{The helping role}

- Comforting through connection and relationship

- Providing comfort measures

- Managing pain, selecting appropriate strategies for pain management and control

- Being with the patient, available without being intrusive

- Maximising the patient's control over their recovery

- Providing comfort and communication

- Providing information for the patient and family 
- Guiding patients through emotional and developmental changes

- Providing adequate stimulation, distraction and rest while limiting disruption

- Comforting through familiar rituals and routines

- Caring for patients' families, ensuring that the family can be with the patient, providing the family with information and support and encouraging family involvement in caregiving activities

\section{Organisational and work-role competencies}

- Co-ordinating, ordering and meeting multiple patient needs and requests: setting priorities

- Building and maintaining a therapeutic team to provide optimum therapy

- Coping with staff shortages and high turnover

- Recognising clinical talent and skilled clinicians and marshalling these for the particular situation

- Modulating one's emotional response and facilitating the social climate

- Preventing hazards in a technological environment by performing practical assessments, engaging in safety work and using equipment and interpreting its performance 


\section{References}

Adam, E. (1980). To be a nurse. Philadelphia: Saunders.

Argyris, C., \& Schon,D. (1974). Theory in practice. San Francisco: Jossey-Bass

Aiken, L.H., Clarke, S.P., Sloan, D.M., Sochalski, J.A., Busse, R., Clarke, H., Giovannetti, P., Hunt, J., Rafferty, A.M., \& Shamian, J. (2001). Nurses' reports on hospital care in five countries. Health Affairs, May/June, 20 (1), 172-88.

American College of Surgeons. (1999). Physicians as assistants at surgery: 1999 study. Available at: http//www.facs.org/dept/hpa/pubs/aas99.pdf

American Nurses Association (ANA). (1998a). The role of the RN in managed care. Nursing World: Trends and Issues, 3 (4).

Available at: http://nursingworld.org/readroom/nti/9804nti.htm

American Nurses Association (ANA). (1998b). Looking for quality patient outcomes: The American Nurses Credentialling Center's Magnet Programme recognises excellence. Nursing World: Trends and Issues, 3 (4).

Available at: http://nursingworld.org/readroom/nti/9807nti.htm

Andrews, S. (1991). New roles, old needs. Nursing Times, 87 (19), p.18. 
Appendices to the Journals of the House of Representatives (AJHR).

1897, H-22, Sess. II, Vol. III, p.7-13.

1902, H-22, Sess. III, Vol. III, p.3.

1903, H-22, Sess. I, Vol. III, p.40.

1905, H-22, Sess. III, Vol. IV, p.6.

1907, H-22, Sess. III, Vol. IV, p.54.

1908, H-22, Sess. IV, No. V, p.12; H-22A, p.13-14.

1909, H-22, Sess. II, No. V, p.6, p.10; H-22A, p.38.

1910, H-22, Sess. III, No. III, p.10.

1911, H-31, Sess. IV, No. IV, p.4; p.8; p.50; p.72; p.77-78, p.183.

1912, H-31, Sess. II, No. IV, p. 4; p.21; p.30. 
Appendices to the Journals of the House of Representatives (AJHR).

1914, H-31, Sess. IV, No. III, p.7; p. 60; H-32, p. 2.

1916, H-7; Sess. II. No. II, H-31, p.6.

1917, H-31, Sess. III, Vol. II, p.6.

1918, H-31, Sess. IV, No. II, p.1-8.

1919, H-31, Sess. VI, Vol. II, p.10-11.

1919, Report of the Commission of Inquiry into the 1918 Influenza

Epidemic,

1919, H-31A, p.1-42.

1920, H-31, Sess. I, Vol. II, p.2-3, 10.

1922, H-31, Sess. IV, No. II, p. 28.

1922, H-31A, Venereal Disease in New Zealand, Sess. IV, No. II, p.1-27.

1929, H-31, Sess. II, Vol. III, p. 23.

1930, H-31, Sess. III, No. III, p.13.

1934, H-31, Sess. IV, Vol. III, p.4, p.6, p. 19.

1935, H-31, Sess. V, No. III, p.40, p.53, p. 56-57.

1936, H-31, Sess. I, No. III, p.41-47.

1940, H-31, Sess. II, Vol. III, p.35.

Austin, A.L. (1957). History of Nursing: Source Book. New York: G.P.Putman's.

Audi, R. (1999). The Cambridge Dictionary of Philosophy (2nd ed.). United

Kingdom: Cambridge University Press.

Australia New Zealand College for Anaesthetists. (1998). The assistant for the anaesthetist. Available from

http://www.anzca.edu.au/publicatons/profdocs/profstandards/ps8 98. htm

Barker, P., \& Reynolds, B. (1994). A critique: Watson's caring ideology: The proper focus of psychiatric nursing? Journal of Psychosocial Nursing, 21(5), 17-22.

Beaglehole, E. (1958). Mental Health in New Zealand. Wellington: Price Milburn. 
Bell, C. (1996). Inventing New Zealand: everyday myths of pakeha identity. Auckland: Penguin Books (NZ).

Belgrave, M. (1985). Medical men and lady doctors: The making of a New Zealand profession 1867 to 1941. Unpublished Doctoral Thesis (History), Victoria University of Wellington.

Belgrave, M. (1989). Medicine and the rise of the health professions in New Zealand 1860 to 1939. In L. Bryder (ed.), A Healthy country: Essays on the social history of medicine in New Zealand. Wellington: Bridget Williams Books.

Benner, P. (1984). From novice to expert: excellence and power in clinical nursing practice. Menlo Park: Addison-Wesley.

Benner, P., Tanner, C.A., \& Chesla, C.A. (1996). Expertise in nursing practice: Caring, clinical judgement and ethics. New York: Springer.

Benner, P., Hooper-Kyriakidis, P. \& Stannard, D. (Eds.).(1999). Clinical wisdom and interventions in critical care: a thinking-in-action approach. Philadelphia: W B Saunders.

Bennett, F.O. (1962). Hospital on the Avon: The North Canterbury Hospital Board. Christchurch: N. M. Peryer Limited.

Board of Health. (1974). An Improved system of nursing services in New Zealand. Wellington.

Boyett, J. H., \& Boyett, J.T. (1996). Beyond Workplace 2000: Essential strategies for the new corporation. Plume, ISBN 0452271959

Boyett, J. H., \& Boyett, J.T. (1998). Five trends shaping our lives.

Available at: http://www.jboyett.com/lifestyl.htm

Boylan, A. (1992). Prove your worth: The value of nursing. Nursing Times, 88 (1), 2829. 
Brown M., Masters D., \& Smith B. (1994). Nurses of Auckland: The history of the General Nursing Programme in the Auckland School of Nursing. Hong Kong: Stylus, Condor.

Bryder, L. (Ed.) (1991). A Healthy country: Essays on the social history of medicine in New Zealand. Wellington: Bridget Williams Books.

Buerhaus, P.I., \& Staiger, D.O. (1997). Future of the nurse labour market in the view of health executives in high managed care states. IMAGE: Journal of Nursing Scholarship, 29, 313-318.

Buerhaus, P.I., Staiger, D.O., \& Auerbach, D.I. (2000). Implications of a rapidly ageing registered nurse workforce. Journal of American Medical Association (JAMA). 283 (22): 2948-2954.

Bunkle, P. (1988). Second opinion: The politics of women's health in New Zealand. Auckland: Oxford University Press

Burgess, M.E. (1984). Nursing in New Zealand society. Auckland: Longman Paul.

Burrus, D. (1993). Techno trends: How to use technology to go beyond your competition. New York: Harper Business.

California Nurses Association. (1999). Pew health profession commission taskforce on reforming healthcare workforce regulation: policy consideration for the $21^{\text {st }}$ century. Available at: http://www.igc.apc.org/can/np/pew2.htm

Cambridge Dictionaries (2000). WordNet 1.6 Vocabulary Helper. Available at: http://dictionary.cambridge.org

Campbell, H. (1976). Mary Lambie: A biography. Wellington: Nursing Education and Research Foundation.

Carpenter, H. (1971). An improved system for nursing education for New Zealand. Report of Dr Helen Carpenter, World Health Organisation short-term consultant. Wellington: Department of Health. 
Cartwright, S.R. (1988). The report of the Committee of Inquiry into allegations concerning the treatment of cervical cancer at National Women's Hospital and into other related matters. Auckland: Government Printer.

Chick, N., \& Rodgers, J. (Eds.) (1997). Looking back, moving forward: Essays in the history of New Zealand nursing and midwifery. Palmerston North: Massey University, Department of Nursing and Midwifery.

Chinn, P., \& Jacobs, M.K. (1979). A model for theory development in nursing. Advances in Nursing Science, 1(1), 1-11.

Chinn, P.L., Jacobs, M.K. (1983). Theory and Nursing: a systematic approach. St Louis: C.V.Mosby.

Chinn, P. L. (1991). Looking into the crystal ball: positioning ourselves for the year 2000. Nursing Outlook, 39(6), 251-256.

Chinn, P.L. (1994). A Window of opportunity. Advances in Nursing Science, 16 (4), viii.

Chopoorian, T.J. (1986). Reconceptualizing the environment. In P. Moccia (Ed.), New approaches to theory development. New York: National League for Nursing.

Clark, J. (1998). The International Classification for Nursing Practice project. Online Journal of Issues in Nursing, September.

Available at: http://www.nursingworld.org/mods/mod15/cec2full.htm

Clifford, J.C. (1998). Restructuring: the impact of hospital organisation on nursing leadership. Chicago: American Hospital Publishing.

Clifford, J.C. (1999). Introduction. In P. Benner, P., Hooper-Kyriakidis., \& D. Stannard. (Eds.), Clinical wisdom and interventions in critical care: a thinking-in-action approach. Philadelphia: W B Saunders.

Cochrane, D. (2001). The future healthcare workforce project and its influence on workforce reform in the United Kingdom. Paper presented at the Health Leaders Network, Sydney. March 16, 2001. 
Cocks, E.M. Somers. (1950). A Friend in need. Nurse Maude: Her life and work.

Christchurch: Nurse Maude District Nursing Association.

Coney, S. (1993). Standing in the sunshine: a history of New Zealand women since they won the vote. Adelaide: Viking Griffen Press.

Conroy, M., \& Cochrane, D. (1996). The future healthcare workforce steering group report. University of Manchester.

Conroy, M., \& Cochrane, D. (1999). The future healthcare workforce, Second Report. University of Bournemouth.

Cochrane, D. (2001). The future healthcare workforce project and its influence on workforce reform in the United Kingdom. Paper presented at a seminar of the Health Leaders Network, March 16 $6^{\text {th }}, 2001$.

Crossan, F., \& Robb, A. (1998). Role of the nurse: Introducing theories and concepts. British Journal of Nursing, 7 (10), 608-612.

Cull, H., Weston, P., \& Adams, J. (1999). Inquiry under S.47 of the Health and Disability Services Act 1993 into the provision of chest physiotherapy treatment provided to pre-term babies at National Women's Hospital between April 1993 and December 1994. Wellington: Ministry of Health, Government Printer.

Dacre Craven, F. S. (1984). A Guide to District Nurses. New York, London: Garland Printing (Reprinted). Originally printed by London: Macmillan and Co. in 1889.

Davis, C. (Ed.). (1981). Rewriting nursing history. London: Croom Helm Ltd.

Degeling, P., Sage, D., Kennedy, J., Perkins, R., \& Zhang, K. (1999). A comparison of the impact of hospital reform on medical subcultures in some Australian and New Zealand hospitals. Australian Health Review, 22(4), 172-188.

Department of Health. (1951). Historical development of nursing in New Zealand 1840 to 1950. Wellington: Government Printer. 
Department of Health. (1971). Chronological list of some noteworthy events in the history of New Zealand health services. Historical development of nursing in New Zealand 1840 to 1950. Wellington: Government Printer.

Department of Health. (1974). A health service for New Zealand. Wellington: Government Printer.

De Rosnay, J. (1997). Feedback. In: F. Heylighen, C. Joslyn and V. Turchin (eds.) Principia Cybernetica Web (Principia Cybernetica, Brussels) Available at: URL: http://pespmc1.vub.ac.be/FEEDBACK. html.

Denzin, N.K., \& Lincoln, Y.S. (Eds.). (1994). Handbook of qualitative research. Thousand Oaks: Sage.

Diers, D. (1984). In From novice to expert: excellence and power in clinical nursing practice. Menlo Park: Addison-Wesley.

Disley, B. (1997). In Mental health in New Zealand from a public health perspective. Wellington: Government Printer.

Donahue, M. P. (1985). Nursing: the finest art. Missouri: CV Mosby.

Dow, D. (1991). Springs of charity? The development of the New Zealand hospital system 1876 - 1910. In L. Bryder (Ed.), A Healthy country: Essays on the social history of medicine in New Zealand. Wellington: Bridget Williams Books, pp. 44-64.

Dow, D.A. (Ed). (1994). Annotated bibliography for the history of medicine and health in New Zealand. Dunedin: Hocken Library, University of Otago.

Dow, D. (1995). Safeguarding the public health: A history of the New Zealand Department of Health. Wellington: Victoria University Press.

Durie, M.H. (1996). Identity, conflict and the search for nationhood. Australian Psychiatry, 4 (4): 189-193. 
Eakes, G., Burke, M., \& Hainsworth, M. (1998). Middle range theory of chronic sorrow. IMAGE:Journal of Nursing Scholarship, 30 (2), 179-184.

English, I. (1993). Intuition as a function of the expert nurse: A critique of Benner's novice to expert model. Journal of Advanced Nursing, 18, 387-393.

Fagin, C.M. (1997). Introduction. In Life support: Three nurses on the front lines. New York: Little, Brown.

Fahey, L., \& Randall, R.M. (1998). Learning from the future: competitive foresight scenarios. New York: John Wiley.

Gallaher, L. (1997). Expert public health nursing practice: A Complex tapestry. Unpublished masters thesis, Massey University, Palmerston North, New Zealand.

Gauthier, D. (1999). Hobbes's social contract. In C.W. Morris, (Ed.). The social contract theorists: Critical essays on Hobbes, Locke and Rousseau. England: Rowman \& Littlefield, pp. 59-71.

Gerteis, M., Edgman-Levitan, S., Daley, J., \& Delbanco, T.L. (1993). Through the patients eyes: understanding and promoting patient-centered care. San Francisco: Jossey-Bass Inc.

Gibbs, A. (1987). Unshackling the hospitals: Report of the hospitals and related services taskforce. Wellington: Government Printer.

Global Business Network (2000). Scenarios.

Available at: http://www.gbn.org/public/gbnstory/scenarios/index.htm

Goodnow, M. (Ed.). (1938). Outline of nursing history. Philadelphia: W B Saunders.

Gordon, M. (1998, September). Nursing nomenclature and classification system development. Online Journal of Issues in Nursing. Available at: http://www.nursingworld.org/ojin/tpc7/tpc7 1.htm

Gordon, S. (1997). Life support: Three nurses on the front lines. New York: Little, Brown. 
Goreberg, B.D., Alderman, M.C., \& Cruise, M.J. (1991). Social policy statements: guidelines for decision making. International Nursing Review, (38), 11-13.

Griffiths, S. (Ed.). (1999). Predictions. New York: Oxford University Press Inc.

Harbour, L.S., Creekmur, T., DeFelice, J., Doub, M.S., Hodel, A., Tomey, A.M., Petty, C.Y. (1998). Evelyn Adam: Conceptual model for nursing. In Tomey, A.M., Alligood, M.R. (1998). Nursing theorists and their work. Fourth Edition. St Louis: Mosby, pp. 516-528.

Hawe, P., Degeling, D., \& Hall, J. (1995). Evaluation health promotion. Australia: MacLennan \& Petty.

Hayne, Y. (1992). The current status and future significance of nursing as a discipline. Journal of Advanced Nursing, 17 (1), 104-107.

Helg, D. (1995). Profession: Nursing. Geneva: International Red Cross \& Red Crescent Museum.

Henderson, V. (1966). The nature of nursing. New York: Macmillan.

Heylighen, F. (1992). Principles of systems and cybernetics: An Evolutionary perspective. Paper presented at the World Science Conference on Cybernetics and Systems, Singapore. Trappl, R (ed.).

Heylighen, F. (1997). The growth of structural and functional complexity during evolution. In F. Heylighen \& D. Aerts (Eds.), The Evolution of Complexity. (Kluwer, Dordrecht), (in press).

Heylighen, F. (1997b). Self-organisation, emergence and the architecture of complexity. In: F. Heylighen, C. Joslyn and V. Turchin (Eds.), Principia Cybernetica Web (Principia Cybernetica, Brussels).

Available at: http://pespmc1.vub.ac.be/progress.gtml

Heylighen, F. (1998). Basic concepts of the systems approach. Available at: http://pespmcl.vub.ac.be/sysappr.html 
Heylighen, F., \& Joslyn, C. (1992). What is systems theory.

Available at: http;//pespmc1.vub.ac.be/systheor.html

Heylighen, F., \& Joslyn, C. (1996). Cybernetics and systems theory.

Available at: http;//pespmcl.vub.ac.be/cybsysth.html

Hinshaw, A.S. (2001). A continuing challenge: the shortage of educationally prepared nursing facility. Online Journal of Issues in Nursing, 6 (1). Manuscript 3. Also available on: http://www.nursingworld.org/ojin/topic14/tpc14 3.htm

Holdaway, M. (1993, March). Where are the Maori nurses who were to become those efficient preachers of the gospel of health? Nursing Praxis in New Zealand, 8, (1), 25-34.

Howden-Chapman, P., \& Cram, F. (1998). Social, economic and cultural determinants of health. Wellington: Ministry of Health National Health Committee.

Howe, M. (1995). In A. Opie, Beyond good intentions: support work with older people. Wellington: Institute of Policy Studies.

Hoy, D. (1995). Making nursing visible: Report of a feasibility study of nursing classification. Londonborough: NHS Centre for Coding and Classification.

Hudson, A. Jones. (Ed.). (1998). Images of Nurses: perspectives from history, art and literature. Philadelphia: University of Pennsylvania Press.

Hyslop, J., Dowland, J., \& Hickling, J. (1983). Health facts New Zealand. Wellington: Department of Health Management Services and Resource Unit.

Ibid (1922). Dr Hughes to Dr Frengley, $6^{\text {th }}$ October.

International Council of Nurses. (1969). Focus on the future: Proceedings of the 14th Quadrennial Congress of the International Council of Nurses in Montreal. Karger, Switzerland.

International Council of Nurses. (1984). Succeeding together. Switzerland: International Council of Nurses. 
International Council of Nurses. (2001). International Classification for Nursing

Practice project (ICNP). Available at: http://www.icn.ch/inp.htm

i SOURCE National Breast Cancer Centre. (2000). Specialist breast nurses: An

evidence-based model for Australian practice. Specialist Breast Nurse Project

Team. Australian Commonwealth Government.

Jamieson, E.M., \& Sewall, M. (1940). Trends in nursing history and their relationship to world events. Philadelphia: W.B. Saunders.

Johnson, D. (1974, Sept - Oct). Development of theory: A requisite for nursing as a primary health profession. Nursing Research, 23(5), 372-377.

Johnson, D.E. (1980). The behavioural system model for nursing. In JP Riehl and C.Roy (eds.), Conceptual models for nursing practice ( $2^{\text {nd }}$ ed.). New York: AppletonCentury-Crofts.

Johnson, M., \& Maas, M. (1997). Nursing outcomes classification (NOC): Iowa intervention project. ( $2^{\text {nd }}$ ed.) St Louis: Mosby-Year Book.

Johnstone, M.J. (1989). Bioethics: A Nursing perspective. Sydney: W. B. Saunders/Bailliere Tindall.

Joslyn, C., \& Turchin, V. (1993). Model. In F. Heylighen, C. Joslyn and V. Turchin (eds.), Principia Cybernetica Web (Principia Cybernetica, Brussels).

Available at: http://pespmc1.vub.ac.be/MODEL.html

Journal of Department of Public Health, Hospitals and Charitable Aid, 1917-20.

$1917,78,172-212$ 
Kai Tiaki: The Journal of the Nurses of New Zealand, 1908 - 1929.

1908, I (1), January, 1, 8; 1 (2), April, 28, 36; 1 (4), October, 113, 118. 1909, II (1), January, 4, 16; II (2), April, 61; II (3), July, 104-105; II (4), October, 137, 164.

1910, III (1), January, 28; III (2), April, 58; III (3), July, 100, 103; III (4), October, 168.

1911, IV (1), January, 20, 28; IV (2), April, 76; IV (3), July, 108, 109, 110, 130, 137; IV (4), October, 162.

Kai Tiaki: The Journal of the Nurses of New Zealand, 1908 - 1929, continued.... 1912, V (1), January, 25, 26; V (3), July, 76; V (4), October, 97, 99. 1913, VI (1), January, 12, 13, 22; VI (2), April, 73; VI (3), July, 113, 155; VI (4) October, 150-154.

1914, VII (1), January, 45-46; VII (2), April, 82; VII (3), July, 112, 120, 124, 129; VII (4), October, 156.

1915, VIII (1), January, 1; VII (2), April, 88; VII (3), July, 132; VII (4), October, 163, 233.

1920, XIII (4), October, 1-10.

1921, XIV (1), January, 19; XIV (4), April, 198.

1922, XV (1), January, 28.

1923, XVI (2), April, 77.

1927, XX (3), July, 123; XX (4), October, 194.

Kane and Associates. (2001). Strategic thinking and use of scenarios. Available at: http://www.kaneandassociates.com/article3.htm 
Katz, R. (1984). Empowerment and synergy: Expanding the community's health resources. New York: Hayworth Press.

Kasper, C.E. (1995). Going through the motions: the ethics of process. Journal of Cardiovascular Nursing, 9 (3), 62-67.

Kassirer, J.P. (1995). The next transformation in the delivery of health care. New England Journal of Medicine, 332, 5204 (Medline).

Keith, J. M. (1988). Florence Nightingale: Statistician and consultant epidemiologist. International Nursing Review, 35 (5), 147-150.

Kelly, L.Y., \& Joel, L.A. (1996). The nursing experience: Trends, challenges and transitions. ( $3^{\text {rd }}$ ed.). New York: McGraw-Hill.

Kelsey, J. (1995). The New Zealand experiment: a world model for structural adjustment. Auckland: Auckland University Press.

Kendall, J. (1992). Fighting back: promoting emancipatory nursing action. Advances in Nursing Science, 15 (2), 1-15.

Kendall, S., \& Corbett, D. (1990). New Zealand military nursing: a history of the Royal New Zealand nursing corps, Boer War to the present day. Auckland:

Kerfoot, K.M. (1997). Role redesign: What has it accomplished? Online Journal of Issues in Nursing, December.

Available at: http://www.nursingworld.org/ojin/tpc5/tpc5 3.htm

King, M. (1983). Maori: A Photographic and social history. Auckland: Heinemann.

Kitson, A. (1996). Does nursing have a future? eBMJ.

Available at: http://www.bmj.com/cgi/content/full/313/7072/1674

Kitson, A. (1997, April). Using evidence to demonstrate the value of nursing. Nursing Standard, 11(28), 34-39.

Knepfer G., \& Johns, C. (1989). Nursing for life. Sydney: Pan Books. 
Kricker, A., \& Jelfs. P. (1996). Breast cancer in Australian women 1921-1994.

Commonwealth of Australia: National Breast Cancer Centre.

Available at: http://www.nbcc.org.au

Labelle, H. (1985). Address at the ICN Congress, Tel Aviv, Israel, reported in Profession: Nurse. Publication for an exhibition in the International Red Cross \& Red Crescent Museum.

Lambie, M. (1951). Historical development of nursing in New Zealand, 1840 - 1950. Wellington: Department of Health.

Lambie, M. (1956). My Story: memoirs of a New Zealand nurse. Christchurch: N. M. Peryer Limited

Laszlo, E. (1996). The systems view of the world: a holistic vision for our time. New Jersey: Hampton Press.

Latimer, R.J., McKay, D.N., Heggie, E.G., Ward, J.T., Gilbert, H.H., Robb, J.H., \& Robb, D. (1969). Health administration in New Zealand. Wellington: New Zealand Institute of Public Administration.

Laubacher, R. J., Malone, T.W., \& MIT Scenario Working Group. (1997). Two scenarios for the $21^{\text {st }}$ century organisations: Shifting networks of small firms or all-encompassing "virtual countries".

Available at: http://ccs.mit.edu/21c/21CWP001.html

Lawler, K. (1999). Specialism: The debate goes on. Professional Nurse, 14(9), 601.

Litchfield, M (1999). Practice Wisdom. Advanced Nursing Science, 22 (2), 62-73.

Litchfield, M. (1998). The scope of advanced nursing practice. Nursing Praxis in New Zealand, 13 (3), 13-24.

Lower, M.S. \& Burton, S. (1989). Measuring the impact of nursing interventions on patient outcomes: The challenge of the 1990s. Journal of Nursing Quality Assurance, 4 (1), 27-34. 
Lynaugh, J.E. (1999). Introduction. In P. Benner, P. Hooper-Kyriakidis, \& D. Stannard (Eds.), Clinical wisdom and interventions in critical care: a thinking-in-action approach. Philadelphia: W B Saunders.

Maclean, H. (1932). Nursing in New Zealand: history and reminiscences. Wellington: Tolan Printing Co.

Maclean, F.S. (1964). Challenge For Health. A history of Public Health in New Zealand. Wellington: Government Printers.

Mallison, M.B. (1990). Editorial: Access to invisible expressways. American Journal of Nursing, 90 (9), 7.

Mann, J. (1998). A lot of care, but little credit. Reflections, First Quarter, 38-38.

Manthey, M. (1985). In T.M. Schorr \& A. Zimmermann (Eds.), Making choices: Taking chances. St Louis: C.V.Mosby.

McCloskey, J.C., \& Bulechek, G.M. (1996). Nursing interventions classification (NIC), Iowa intervention project ( $2^{\text {nd }}$ ed.). St Louis: Mosby-Year Book.

McCloskey, J.C., \& Bulechek, G.M. (2000). Nursing interventions classification (NIC), Iowa intervention project ( $3^{\text {rd }}$ ed.). St Louis: Mosby-Year Book.

McCorduck, P., \& Ramsey, N. (1996). The futures of women: scenarios for the $21^{\text {st }}$ century. New York: Warner Books.

McCullagh, C. Behan. (1984). Justifying Historical Descriptions. Cambridge: Cambridge Press.

McFarlane, J. (1977). Essays on nursing. London: Kings Fund Project Paper.

McKegg, A.H. (1991). "Ministering Angels": The government backblock nursing service and the Maori health nurses, 1909-1939. Unpublished masters thesis, University of Auckland, Auckland, New Zealand.

Meerabeau, L., Casey, A., \& Old, S. (1997). Language for research and practice: The English experience. Paper presented at Nursing Informatics Conference. Stockholm. 
Meleis, A.I. (1991). Theoretical nursing: development and progress ( $2^{\text {nd }}$ ed.). Philadelphia: J.B.Lippincott.

Merriam, S.B. (1988). Case study research in education: a qualitative approach. San Francisco, CA: Jossey-Bass.

Ministerial Taskforce on Nursing. (1998). Report of the Ministerial Taskforce on Nursing:Releasing the potential of nursing. Wellington: Government Printer.

Ministry of Health. (1995). Annual Report. Wellington: Government Printer.

Ministry of Health. (1992). Green and white paper. Wellington: Government Printer.

Ministry of Health. (1997). Mental health in New Zealand from a public health perspective. Wellington: Government Printer.

Ministry of Health. (1998a). Whaia Te Whanaungatanga Oranga Whanau: The wellbeing of whanau: a background paper. Wellington: Government Printer.

Ministry of Health. (1998b). Progress on health outcome targets: The state of the public health in New Zealand. Wellington: Government Printer.

Ministry of Health. (1999a). Health expenditure trends in New Zealand 1980-1998. Wellington: Government Printer.

Ministry of Health. (1999b). Rural health policy: meeting the needs of rural communities. Wellington: Government Printer.

Ministry of Health. (2000a). Health needs assessment for New Zealand. Wellington: Government Printer.

Ministry of Health. (2000b). Our health, our future: The health of New Zealand. Hauora pakari, koiora roa. Wellington: Government Printer.

Ministry of Health. (2000c). The New Zealand health strategy. Wellington: Government Printer.

Ministry of Health. (2000d). Towards clinical excellence: A framework for credentialing. Wellington: Government Printer. 
Ministry of Health. (2001). The burden of disease and injury in New Zealand. Wellington: Government Printer. Public Health Intelligence, Occasional Bulletin No.1.

Moccia, P. (1988). At the fault line: Social activism and caring. Nursing Outlook, 36 (1), 30-33.

Montgomery, A. (2001). Of vision, virtue, valour and victory. British Journal of Perioperative Nursing, 11 (2), 84-89.

Morris, C.W. (1999). The social contract theorists: critical essays on Hobbes, Locke and Rousseau. England: Rowman \& Littlefield.

Mortensen, R. (Ed.). (1996). International Classification for Nursing Practice (ICNP) with telenurse introduction. Copenhagen: The Danish Institute for Health and Nursing Research.

Munro, J. (1996). The story of Suzanne Aubert. Auckland: Auckland University Press.

Munslow, A. (1997). Deconstructing history. London: Routledge Press.

Murray, R., Beckmann., \& Zentner, J. Proctor. (1979). Nursing concepts for health promotion. Englewood Cliffs, N.J: Prentice-Hall.

N.H.S. (2000). A health service of all the talents: Developing the NHS workforce. Consultation document on the review of workforce planning. London: Department of Health.

National Action Group. (1988). Review of the preparation and initial employment of nurses, No.1. Wellington: Government Printer.

National Council of State Boards of Nursing. (1994). In L.Y. Kelly., \& L.A. Joel. (1996). The nursing experience: Trends, challenges and transitions $\left(3^{\mathrm{rd}}\right.$ ed.). New York: McGraw-Hill.

National Health Committee. (1998). The social, cultural and economic determinants of health in New Zealand: Action to improve health. Wellington: Government Printer. 
Naylor, M.D., Munro, B.H., \& Brooten, D.A. (1991). Measuring the effectiveness of nursing practice. Clinical Nurse Specialist, V, 210-215.

Neill, J.O.C. (1961). Grace Neill: The story of a remarkable woman. Christchurch: N.M. Peryer Ltd.

Newman, M. (1986). Health as expanding consciousness. St Louis: C.V.Mosby.

New Zealand Guidelines Group. (1999). Guidelines for primary care providers: Early detection of breast cancer. Available at: http://www.nzgg.org.nz/library.cfm.

New Zealand Journal of Health \& Hospitals.

1920, III (3), March, 20

1921, IV (1), January, 90-91.

New Zealand Nurses Organisation. (1998). Standards for Nursing Practice.

New Zealand Nursing Journal, 1929 - 1993.

1936, XXIX, November, (6), 199, 240.

1937, XXX, March, (2), 37, 60-61; November, (6), 32-37.

1942, XXXV, February, (2), 1; June, (6), 1; September, (8), 1; November, (10),

1; December, (11), 1, 295-96.

1943, XXXVI, March, (3), 1; May, (6), 1, 198-199; June, (6), 1; October, (10), 1.

Newton, E. (1965). The case for historical research. Nursing Research, 14 (1), 20-26.

New Zealand Official Yearbook by E.J Von Dadelszon, Wellington: Government

Printer.

1891, 141, RNZ 319 N5.

1896, 140, RNZ 319 N5.

1899, 60-61, RNZ 319B NS. 
New Zealand Parliamentary Debates. (NZPD).

1901, 116, July 1- July 26,116, Mr Kelly, Taranaki.

New Zealand Yearbook. (1999). Available at:

http://www.stats.govt.nz/domino/exte.../Yearbook+5_population+1999+5+population?o

pe

Nightingale, F. (1859). Notes on nursing: What it is, and what it is not. London: Harrison \& Sons, re-published by Edinburgh: Churchill Livingstone (1980).

Nursing Council of New Zealand (1999). Disciplinary Provisions of the Nurses Act

1977. Available at: http://www.nursingcouncil.org.nz/pub.html

Nursing Council of New Zealand. (2001). Guidelines for competence-based practising certificates for registered nurses. November.

Available at: http://www.nursingcouncil.org.nz/pub.html

Nursing Council of New Zealand. (2001). KPMG strategic review of undergraduate nursing education: report to Nursing Council of New Zealand. NCNZ00FinalReport0502-SCR.

O’Neil, E.H., \& the Pew Health Professions Commission. (1998). Recreating Health Professional Practice for a New Century. San Francisco: Pew Health Professions Commission. Available: http://futurehealth.ucsf.edu/compubs.html

Orem, D.E. (1988). Nursing: concepts of practice ( $3^{\text {rd }}$ ed.). New York: McGraw-Hill.

Oulton, J. (1997). International trends in nursing professional development. International Nursing Review, 44 (2), 47-48.

Parfitt, B. (1995). The need for a universal definition of nursing. Health Care Analysis, Journal of Health Philosophy and Policy, 3 (1), 53-60. 
Parkes, C.M. (1991). The impact of the medicalisation of New Zealands maternity services on womens experience of childbirth, 1904 - 1937. In L. Bryder (Ed.), A healthy country. Essays on the social history of medicine in New Zealand. Wellington: Bridget Williams Books. pp. 165-180.

Parrott, A. (1995). A modern profession with an outdated image. In Profession: Nurse. Publication for an exhibition in the International Red Cross \& Red Crescent Museum.

Paterson, B. (1989). Making a difference: The lived world of nursing practice in an acute care setting. Masters Thesis, Massey University, Palmerston North.

Paterson, A., \& Marsden, E. (1927). Physical Growth and Mental Attainment of School Children, Appendices to the Journals of the House of Representatives, 1927, H$31,54-63$.

Paterson, J.G., \& Zderad, L.T. (1976). Humanistic nursing. New York: G.P. Putman Sons.

Peach, J. (1999). The professional development programme: Achievements and outcomes. Professional Leader, 5 (1), 6-9.

Pere, R.R. (1984). Te oranga o te whanau: the health of the family. In Hui Whakaoranga: Maori Health Planning Workshop. Wellington: Department of Health.

PEW Commission. (1998). Recreating Health Professional Practice for a New Century. The $4^{\text {th }}$ report of the Pew Health Professions Commission. December. Available at: http://futurehealth.ucsf.edu/pewcomm/competen.html

Platt, C. (1998). The museum of nanotechnology.

Available at: http://www.wired.com/wired/scenarios/museum.html

Pomare, E. (1995). Hauora: Maori Standards of Health III: A study of the years 1970 1984. Wellington: Huia Publishers. 
Pool, D.I. (1977). Is New Zealand a healthy country? The centenary of Dr Alfred

Newman's affirmation "That it is yet the healthiest on the face of the globe. New Zealand Population Review, 8 (2), 2-27.

Pool, D.I. (1977). The Maori population of New Zealand 1769-1971. Auckland:

Auckland University Press.

Pope, J.H. (1884). Health for the Maori: a manual for use in native schools. Wellington. Porter-O'Grady, T., \& Krueger Wilson, C. (1998). The health care team book. St Louis: Mosby.

Price Waterhouse Cooper. (1999). Smaller world bigger expectations. Healthcast 2010. Privacy Commissioner. (1996). Casework 2049.

http://www.microsoft.com/windows/ie/ie5/download/ieupdate.htm

Public Health Commission. (1995). Progress on health outcome targets: the state of the public health in New Zealand. Wellington: Government Printer.

Pybus, M. (Ed.). (1983). The nurse in the Community: Community Health Nursing in New Zealand. Auckland: McGraw-Hill.

Rattray, J. (1961). Great days in New Zealand nursing. Wellington: A.H. \& A.W. Reed.

Rice, G. W. (1988). Black November: The 1918 Influenza Epidemic in New Zealand. Wellington: Allen \& Unwin, Historical Branch.

Rice, G.W. (1991). Public health in Christchurch, 1875 - 1910: mortality and sanitation. In L. Bryder (Ed.) A Healthy Country: Essays on the social history of medicine in New Zealand, pp. 85-108. Wellington: Bridget Williams Books. pp. 85-108.

Rice, G.W. (Ed.). (1992). The Oxford history of New Zealand. $2^{\text {nd }}$ edition. Wellington: Oxford University Press.

Ripstein, A. (1999). The general will. In C.W. Morris (Ed.). The social contract theorists: Critical essays on Hobbes, Locke and Rousseau. England: Rowman \& Littlefield. pp. 219-235. 
Robbins, S.P. (1990). Organisation theory: Structure, design and applications $\left(3^{\text {rd }}\right.$ ed.). New Jersey: Prentice Hall.

Rogers, M.E. (1970). An introduction to the theoretical basis of nursing. Philadelphia: F.A. Davis.

Rogers, M. (1972). Nursing: To be or not to be? Outlook, 20, 42-46.

Roy, C. (1984). Introduction to nursing: An adaptation model ( $2^{\text {nd }}$ ed.). Englewood Cliffs, NJ: Prentice-Hall.

Royal College of Physicians. (2000). Hospital Doctors Under Pressure: New Roles for the Health Care Workforce.

Available at: http://www.rcplomdon.ac.uk/pubs/wp hdup.htm

Russell, R.L. (1991). Are we asking the right questions. In G. Gray \& R. Pratt (Eds.), Towards a discipline of nursing. (pp.73-93). Melbourne: Churchill Livingston.

Salmon E.B. (1982). A Profession in transition. Wellington: The C.L.Bailey Nursing Education Trust.

Sargison, P.A. (1986). From candles to computers: A bibliography of printed sources on the history of nursing in New Zealand. Wellington: Nursing Education and Research Foundation.

Sargison, P. (1993). Notable women in New Zealand health: Te Hauora ki Aotearoa: Ona Wahine Rongonui. Auckland: Longman Paul.

Schofield, M. (1996). The Future Healthcare Workforce: the steering group report. Bournemouth: Bournemouth University.

Schorr, T.M., \& Zimmermann, A. (Eds.). (1985). Making choices: Taking chances. St Louis: C.V.Mosby.

Schwartz, P. (1996). The art of the long view: planning for the future in an uncertain world. New York: Currency Doubleday.

Scott, C. (1998). Specialist practice: Advancing the profession. Journal of Advanced Nursing, 28 (3), 554-62. 
Scott, D. (Ed.). (1976). The Story of Auckland Hospital 1877 to 1964. Auckland: Medical Historical Library Committee of the Royal Australian College of Physicians in New Zealand.

Seedhouse, D. (1997). Health: the foundations for achievement. Wiltshire: John Wiley.

Seedhouse, D. (2000). Practical nursing philosophy: The universal ethical code. New York: John Wiley.

Senge, P., Kleiner, A., Roberts, C., Ross, R., Roth, G., \& Smith, B. (1999). The dance of change the challenges of sustaining momentum in learning organisations. New York: Currency Doubleday.

Shaw, S. (1986). Mind over mindset. Paper presented at the Norman Peryer Forum. NERF Studies in Nursing, No. 14. Wellington: Nursing Education and Research Foundation.

Simpson, T. (1997). The immigrants: the great migration from Britain to New Zealand 1830 to 1890. Auckland: Godwit.

Smith, J.A. (1983). The idea of health: implications for the nursing professional. New York: Teachers College, Columbia University.

Smith, M.G., \& Shadboldt, Y.T. (1984). Objects and outcomes: New Zealand Nurses Association 1909-1983. Wellington: New Zealand Nurses Association.

Smith, R. (Ed). (1991). The health of the nation: The BMJ View. Plymouth: Latimer Trend.

Snow, Terry (Ed.). (1998). The New Zealand Herald book of the century. Wellington: Hucheson, Bowman \& Stewart.

Sochalski, J., Aiken, L.H., \& Fagin, C.M. (1997). Hospital restructuring in the United States, Canada and Western Europe: an outcome research agenda. Medical Care, 35 (10), OS13-OS25. 
Staiger, D.O., Auerbach, D.I., \& Buerhaus, P.I., (2000, Sept/Oct). Expanding Career Opportunities for Women and the Declining Interest in Nursing as a Career. Nursing Economic\$.18(5), 230-236.

Stake, R.E. (1981). Case study methodology: an epistemological advocacy. In W.W. Welsh (ed.) Case study methodology in educational evaluation. Minneapolis: Minnesota Research and Evaluation Center.

Statistics New Zealand. (2000). New Zealand income survey 2000. Also Prison inmates 1880-1998. Available at: http://www.stats.govt.nz/

Steelman, V.M., Bulechek, G.M., McCloskey, J.C. (1994, November). Towards a standardised language to describe perioperative nursing. AORN Journal, 60 (5), 786-795.

Stocks, M. (1960). A hundred years of District Nursing. London, George Allen \& Unwin, Ltd.

Storlie, F. (1970). Nursing and the social conscience. New York: Appelton-CenturyCrofts.

Styles, M.M. (1982). On nursing: Towards a new endowment. St. Louis: CV Mosby.

Sutch, W.B. (1971). The responsive society in New Zealand. Christchurch: Whitcombe \& Tombs.

Suter, K. (1999). Globalisation, governance, sustainable development: Final report of the conference of the Club of Rome. Vienna, Austria.

Available at: http://www.clubofrome.org.archive/declaration.html\#start

Tauroa, P. (1990). The Collins Maori Phrase Book. Auckland: William Collins Publishers Ltd.

The Center for the Health Professions.(1995).Critical Challenges: Revitalising the health professions for the twenty-first century. The Third Report of the Pew Health Professions Commission, August.

Available at: http://www.futurehealth.ucsf.edu/summaries/challenges.html 
The Concise Oxford Dictionary. (1990). $8^{\text {th }}$ edition. England: Oxford University Press.

The New Zealand Nursing Journal

1935, Vol. XXVIII, May, 62, 78; July, (6), 107; November(11),199.

1936, Vol. XXIX, January (1),

1937, Vol. XXX, February, (1), 8, 37; March, (3), 60,61; December (11), 326,

329.

1941, Vol. XXXIV, November, (10), 1.

1942, Vol. XXXV, February, (2), 1; June, (6),1; September (8), 1; November

(10), 1; December, (11), 295.

1943, Vol. XXXVI, February, (1), ; March, (2), ; May, (5), ; June (6), 123,124; October, (10), .

1944, Vol. XXXVII, July, (7), 44; October (10), 219, 220, 233.

The Little Oxford Dictionary. (1998). Rev. 7th edition. United Kingdom: Oxford University Press.

The Royal New Zealand College of General Practitioners. (2000). Detection and Management of Young People at Risk of Suicide.

Available at: http://www.rnzcgp.org.nz/guidelines/YS/ys.htm

Travelbee, J. (1971). Interpersonal aspects of nursing ( $2^{\text {nd }}$ ed.). Philadelphia: F.A.Davis.

Tripp-Reimer, T. (1984). Reconceptualising the construct of health: integrating emic and etic perspectives. Research in Nursing and Health, 7: 101-109.

Tripp-Reimer, T., Woodworth, G., McCloskey, J.C., \& Bulachek, G. (1996). The dimensional structure of nursing interventions. Nursing Research, 45, 10-17.

Tomey, A.M., Alligood, M.R. (1998). Nursing theorists and their work. Fourth Edition. St Louis: Mosby.

Toynbee, A. (1934). A study of history. London: Oxford University Press. 
Turchin, V. (1997). Agent. In: F. Heylighen., C. Joslyn., and V. Turchin. (Eds.), Principia Cybernetica Web (Principia Cybernetica, Brussels).

Available at: URL: http://pespmc1.vub.ac.be/AGENT.html.

Turner, B.S. (1987). Medical power and social knowledge. London: Sage.

United States of America Government. (1999). Futurework: Trends and challenges for work in the $21^{\text {st }}$ century.

Available at: http:Ilwww.dol.gov/dol/asp/public/futurework/report.htm

Ursin, G., Bernstein, L., \& Pike, M.C. (1994). Breast cancer. Cancer surveys 19/20:241-

64. Available at: http://www.nbc.org.au/pages/info/resource/nbccpubs/bc2194/longtrbc.htm.

Van Der Heijden, K. (1996). Scenarios: The art of strategic conversation. Chichester: John Wiley.

Vester, F. (1999). Cybernetic System Management as a guarantor of sustainable development. Paper presented at Globalisation, governance, sustainable development conference of the Club of Rome, Vienna.

Available at: http://www.clubofrome.org.archive/declaration.html\#start

Wack, P. (1985). Scenarios: Uncharted waters ahead. Harvard Business Review, 63 (5), 72-89.

Waltz, C.F., \& Strickland, O.L. (1988). Measurement of nursing outcomes: measuring client outcomes, 1. New York: Springer.

Waltz, C.F., \& Strickland, O.L. (1990). Measurement of nursing outcomes: measuring client self-care and coping skills, 4. New York: Springer.

Weisbord, M.R., \& Janoff, S. (1995). Future search: An action guide to finding common ground in organisations and communities. San Francisco: Berrett-Koehler.

Whyte, S. (2000). The specialist nurse: a classification system. Contemporary Nurse, 9 (1), 6-15. 
Wilkes, L., \& Wallis, M. (1993). The 5 C's of caring: The lived experiences of student nurses. Australian Journal of Advanced Nursing, 11 (1), 19-25.

Wilkinson, J.M. (2000). Nursing diagnosis handbook with NIC interventions and NOC outcomes $\left(7^{\text {th }}\right.$ ed.). New Jersey: Prentice Hall Health.

Wilkinson, L. (1998). How to build scenarios.

Available at: http://www.wired.com/wired/scenarios/build.html

Williams, B.G. (2000). The primacy of the nurse in New Zealand 1960s-1990s: Attitudes, beliefs and responses over time. Unpublished doctoral thesis, Victoria University of Wellington, Wellington, New Zealand.

Wilson, C. (1999). The impact of medical technologies on the future of hospitals. $e B M J$ (British Medical Journal).

Available at http://www.bmj.com/cgi/content/full/319/7220/1287

Wilson, I. (1975). From scenario thinking to strategic action.

Available at: http://horison.unc.edu/projects/seminars/futurising/action.asp

Wood, P. (1990). Nursing: Progress through partnership 1921-1991. Canberra: Department of Community Services and Health/Australian Government Publishing Service.

Wood, P.J. (1992, March). Efficient preachers of the gospel of health: the 1898 scheme for educating Maori nurses. Nursing Praxis in New Zealand, 7 (1), 12-21.

Wood, P.J. (1994, July). An exemplar from the past: Florence Nightingales influence on a New Zealand nurses practice. Nursing Praxis in New Zealand, 9 (2), 38-39.

Woodward, A., \& Kawachi, I. (1998). Why should we reduce health inequalities? Paper prepared for the National Health Committee. Wellington: Government Printer.

World Health Organisation. (1986). Ottawa Charter for Health Promotion.

Available at: http://www.who.dk/policy/ottawa.htm

Wright S. G. (1989). Changing nursing practice. London: Edward Arnold, Hodder Headline Group. 
Wright-St Clair, R. E. (1987). A history of the New Zealand Medical Association.

Wellington: Butterworths.

Yin, R.K. (1984). Case study research: Design and methods. Newbury Park, CA: Sage.

Yin, R.K. (1994). Case study research: Design and method (2nd ed.). Thousand Oaks, CA: Sage. Applied Social Research Methods Series, 5.

Yura, H. \& Walsh, M.B. (1988). The Nursing Process, $5^{\text {th }}$ Ed. Norwalk: AppletonCentury-Crofts. 